\title{
Radar Backscatter Modeling Based on Global TanDEM-X Mission Data
}

\author{
Zur Erlangung des akademischen Grades einer
}

\section{DOKTOR-INGENIEURIN}

von der Fakultät für

Elektrotechnik und Informationstechnik der Universität Fridericiana Karlsruhe (TH)

genehmigte

\section{DISSERTATION}

von

\section{M.Sc. Paola Rizzoli}

geb. in Sarnico, Bergamo, Italien

Tag der mündlichen Prüfung:

05. 10. 2018

Hauptreferent:

Prof. Dr.-Ing. habil. Alberto Moreira

Korreferent:

AO Univ. Prof. Dr. Helmut Rott 

È dunque sognando ad occhi aperti, io credo, che vivi intensamente; ed è ancora con l'immaginazione che puoi trovarti a competere persino con l'inattuabile. E qualche volta ne esci anche vincitore.

Walter Bonatti 


\section{Acknowledgements}

I would like to thank Prof. Dr. Alberto Moreira, director of the DLR Microwaves and Radar Institute, for his constant guidance throughout the development of this work. Thank you to my supervisors, Dr. Manfred Zink and Dr. Benjamin Bräutigam, for their great support and for constantly motivating me to conclude the $\mathrm{PhD}$. My gratitude goes to Prof. Dr. Helmut Rott for his highly valuable technical support and for accepting to be the co-reviewer of this thesis.

I also would like to thank all the other members of my research group for their support, the interesting discussions, and their availability to review the manuscript: Michele Martone, Christopher Wecklich, Carolina Gonzalez, Andrea Pulella, José-Luis Bueso-Bello, Paolo Valdo, and Nicola Gollin.

I thank all the colleagues at DLR who helped me to improve the quality of the thesis and of the final presentation and to organize all the formalities for the $\mathrm{PhD}$ defense at the University of Karlsruhe. In particular, I am grateful to Dr. Daniela Borla Tridon, Dr. Francescopaolo Sica, Dr. Matteo Nannini, Dr. Michelangelo Villano, Dr. Claudio Castellini, Dr. Muriel Pinheiro, Dr. Felipe Queiroz de Almeida, Dr. Sigurd Huber, Dr. Giuseppe Parrella, and Dr. to be Georg Fischer.

Moreover, a sincere acknowledgement goes to all other colleagues at DLR and to all my friends in Munich for their company in the everyday life. I specially thank my German connection and dearest friends Uli and Alex, for the great time together and for unlocking the secrets of the German (and Bavarian) society. Thank you also to Elisa and Michele for their long-lasting friendship, that I allow myself to call brotherhood. And thank you to Nadia, for taking me with her to the top of the mountains and showing me the world from other perspectives.

I am deeply grateful to my parents, my sister, and Indy for always loving and supporting me and sharing good and bad times together.

Finally, I would like to dedicate this work to the memory of my grandmother Peppina, whose unconditioned love will accompany me for the rest of my life.

Paola Rizzoli 


\section{Zusammenfassung}

Radarrückstreuung bezeichnet den Teil eines ausgesendeten elektromagnetischen Signals, der von einem Ziel am Boden wieder zurück zur Antenne gerichtet ist. Die Eigenschaften des zurückgestreuten Signals ändern sich in Abhängigkeit von Frequenz und Polarisation des Radarsignals, der Aufnahmegeometrie, sowie vom Zustand des Erdbodens und der Art der Bodenbedeckung. Informationen über das Radarrückstreuverhalten sind von höchster Wichtigkeit für die Auslegung von SAR-Missionen und werden verbreitet zur Entwicklung wissenschaftlicher Modelle genutzt, beispielsweise bei der Erforschung der Biosphäre und Kryosphäre. Hauptziel dieser Arbeit ist die Auswertung und Nutzung des globalen TanDEM-X-Datensatzes zur Modellierung der Radarrückstreuung im X-Band unter Berücksichtigung unterschiedlicher Aufnahmeparameter und Landnutzungsarten, sowie die Bereitstellung einer Reihe von globalen Rückstreumodellen, die auf aktuellen Daten basieren, für die wissenschaftliche Gemeinschaft. Es wurde ein neuer Ansatz zur statistischen Modellierung der Rückstreuinformation entwickelt, der die Qualität der zugrunde liegenden Messungen berücksichtigt. Daraus ergeben sich gewichtete polynomiale Modelle für die verschiedenen Landnutzungsarten, wie sie in der GlobCover-Karte der ESA definiert sind. Darüber hinaus wird ein eigener Validierungsansatz vorgestellt, mit zusätzlicher Betrachtung der saisonalen Variation der Rückstreuung und einer separaten Analyse des Rückstreuverhaltens des Tropischen Regenwaldes. Der nächste Schwerpunkt ist die Betrachtung des Grönländischen Eisschildes, das gekennzeichnet ist durch das Vorhandensein verschiedener Arten von Schneebedeckung, die von trockenem bis hin zu sehr feuchtem Schnee variiert. Der begrenzte Detailgrad, den die GlobCover Karte in Grönland aufweist (nur eine Klasse für das gesamte Eisschild), erlaubt dort keine verlässliche Modellierung der Rückstreuung. Diese Schwierigkeit lieferte die Motivation für die Entwicklung eines neuen Ansatzes zur Analyse des Informationsgehalts der interferometrischen TanDEM-X-Daten mit dem Ziel, unterschiedliche Schnee-Fazien mit Hilfe des sog. C-Means Fuzzy Clustering Algorithmus zu lokalisieren. Aus dieser Untersuchung konnte die Existenz von vier unterschiedlichen Klassen von Schnee-Fazien abgeleitet werden, deren Eigenschaften anschließend mit Hilfe externer Referenzdaten interpretiert wurden. Die daraus entstandene Karte wurde zur Erstellung eines einfallswinkelabhängigen Rückstreumodells genutzt, separat für jede der vier Klassen, wobei eine modifizierte Version des entwickelten Algorithmus zur Generierung globaler Rückstreumodelle eingesetzt wurde. Darüber hinaus wurde als Nebenprodukt zusätzlich die Eindringtiefe von TanDEM-X in die Eisschicht geschätzt, durch Inversion des von Weber Hoen und Zebker vorgeschlagenen "Ein-chicht-Volumendekorrelationsmodells". Die Ergebnisse wurden mit dem Höhe- 
nunterschied zwischen dem globalen TanDEM-X-DEM und ICESat-Messungen verglichen. Abschließend wird ein neu entwickelter Algorithmus zur Generierung von Rückstreukarten großer Gebiete vorgestellt. Dieser erlaubt unter Verwendung von Rückstreumodellen das Angleichen der erstellten Karten anhand eines Referenzeinfallswinkels, was dann das Füllen verbleibender Lücken ermöglicht, die aufgrund fehlender Eingangsdaten vorhanden sind. 


\section{Abstract}

Radar backscatter represents the portion of a transmitted electromagnetic signal that is redirected back toward the antenna from a target on ground. Its properties change depending on the radar wave frequency and polarization, acquisition geometry, ground cover type, and soil conditions. Backscatter information is of paramount importance for the design of SAR missions and is widely used for the development of scientific models in the fields of, e.g., the biosphere and cryosphere.

The main goal of this work is to exploit the global TanDEM-X SAR data set to model radar backscatter at $\mathrm{X}$ band, considering different acquisition parameters and land cover types and to provide then the scientific community with an up-to-date set of backscatter models at a global scale.

A novel approach for statistically model the backscatter information, which takes into account the quality of the input measurements, has been developed. The results are weighted polynomial models for different land cover types, taken from the ESA GlobCover map. A dedicated validation approach is presented as well, together with additional considerations on backscatter seasonality and a dedicated analysis of backscatter behavior over tropical rainforests.

The attention is then focused on the Greenland Ice Sheet, which is characterized by the presence of different kinds of snow cover, from dry to wet snow. Here, the insufficient level of detail that is provided by the GlobCover map over Greenland (one single class for the entire ice cap) does not allow for a reliable modeling of backscatter. This obstacle set the motivation for developing a new approach for analyzing the information content of interferometric TanDEM-X data, aimed at locating different snow facies by means of the $c$-means fuzzy clustering algorithm. A set of four different snow facies has been derived, and their properties interpreted with the help of external reference data. The obtained map has then been used to generate an incidence angle dependent backscatter model for each snow facies, separately, by using a modified version of the developed algorithm for the generation of global backscatter models. Moreover, as by-product, the penetration depth of TanDEM-X into the ice pack has been estimated as well, by inverting the volume decorrelation single-layer model proposed by Weber Hoen and Zebker. The results have then been compared to the difference in height between the global TanDEM-X DEM and ICESat measurements.

Finally, an algorithm for the generation of large-scale backscatter maps has been developed as well. It requires the use of backscatter models to equalize the output maps to a certain incidence angle of reference and to eventually fill remaining gaps caused by missing input data. 


\section{Contents}

List of Symbols $\quad$ xi

List of Mathematical Operators $\quad$ xvii

List of Acronyms xviii

1. Introduction 1

1.1. Radar in Remote Sensing . . . . . . . . . . . . . . . . . . . . . . . . . 1

1.2. A Historical Overview of Spaceborne SAR . . . . . . . . . . . . 3

1.3. Motivation of the Work . . . . . . . . . . . . . 5

1.4. Thesis Structure . . . . . . . . . . . . . . . . 6

1.5. Original Contribution of the Thesis . . . . . . . . . . . 7

2. Theoretical Background $\quad 9$

2.1. Electromagnetic Waves ... . . . . . . . . . . . . . . . . . . 9

2.1.1. Electromagnetic Plane Waves . . . . . . . . . . . . . 9

2.1.2. Electromagnetic Power Density . . . . . . . . . . . . . 13

2.1.3. Wave Polarization . . . . . . . . . . . . . . . 13

2.1.4. Wave Reflection and Transmission at the Interface . . . . . . 14

2.2. Radar Backscattering . . . . . . . . . . . . . . . . . . . 16

2.2.1. Scattering Matrix . . . . . . . . . . . . . . 16

2.2.2. Scattering from a Point Target . . . . . . . . . . . . 17

2.2.3. Scattering from a Distributed Target . . . . . . . . . . . . 18

2.2.4. Surface and Volume Scattering . . . . . . . . . . . . . . 20

2.2.5. Chapter Remarks . . . . . . . . . . . . . . . . . 21

3. Principles of SAR Imaging and Interferometry 22

3.1. SAR Image Formation . . . . . . . . . . . . . . . . . . . . . . 22

3.1.1. Acquisition Geometry and Resolution . . . . . . . . . . . . . 22

3.1.2. Perspective Deformations . . . . . . . . . . . . . 26

3.1.3. Range Focusing . . . . . . . . . . . . . . . . . . . . . . . . . . 26

3.1.4. Azimuth Focusing . . . . . . . . . . . . . . . . . . . 27

3.1.5. Image Speckle and Multilooking . . . . . . . . . . . . . . 28

3.1.6. SAR Acquisition Modes . . . . . . . . . . . . . . . . . 30

3.1.7. Absolute Calibration of SAR Images . . . . . . . . . . . . 31

viii 
3.1.8. SAR Image Geocoding . . . . . . . . . . . . . . . . . . . . . . . . . . . . . . 32

3.2. SAR Interferometry . . . . . . . . . . . . . . . . . . . 34

3.2.1. From the Acquisition Geometry to the Height Retrieval . . . . . 34

3.2.2. The Interferogram . . . . . . . . . . . . . . . . 36

3.2.3. Spectral Shift . . . . . . . . . . . . . . . . . . . . . . 39

3.2.4. The interferometric Coherence . . . . . . . . . . . . 40

3.2.5. Phase Unwrapping and DEM Generation . . . . . . . . . . 40

3.2.6. Chapter Remarks . . . . . . . . . . . . . . . . . . . . . 43

4. The TanDEM-X Mission and its Global Data Set 44

4.1. TerraSAR-X and TanDEM-X Satellites . . . . . . . . . . . . . . . 44

4.2. Mission Overview . . . . . . . . . . . . . . . . . . . . 45

4.3. TanDEM-X Data: Quicklook Images . . . . . . . . . . . . . . . . 50

5. A New Approach for Statistical Modeling of Radar Backscatter 56

5.1. State of the Art in Radar Backscatter Modeling . . . . . . . . . . . . . 56

5.2. Mapping Radar Backscatter . . . . . . . . . . . . . . . . . . . . 57

5.3. A New Method for Modeling Backscatter . . . . . . . . . . . . . 58

5.3.1. Backscatter Characterization from Single SAR Acquisitions . . 58

5.3.2. Modeling Algorithm . . . . . . . . . . . . . . . . 65

5.3.3. Summary of methods output . . . . . . . . . . . . . . . 69

5.3.4. Discussion upon the Sources of Error . . . . . . . . . . . 71

5.4. Performance Evaluation Approach of Global Backscatter Models . . . . 75

5.4.1. Check for Absolute Offsets . . . . . . . . . . . . . . . . . . 75

5.4.2. Curvature and Slope Accuracy . . . . . . . . . . . . . . . 77

5.4.3. Combination of Different Test Sites . . . . . . . . . . . . 79

5.5. Seasonal and Geographic Backscatter Dependency . . . . . . . . . . 83

5.6. A Large-Scale Study of Tropical Rainforests Backscatter . . . . . . . . 86

5.6.1. Input Test Sites and $\gamma^{0}$ Quicklook Mosaics . . . . . . . . . . 87

5.6.2. Analysis of $\gamma^{0}$ Incidence Angle Dependency . . . . . . . . . 87

5.6.3. Analysis of $\gamma^{0}$ Seasonal Dependency . . . . . . . . . . . 90

6. The Greenland Ice Sheet Case 94

6.1. The Greenland Ice Sheet: an Introduction to the Work . . . . . . . . . . 95

6.2. Fuzzy Clustering for Snow Facies Classification . . . . . . . . . . . . . 97

6.2.1. The c-Means Fuzzy Clustering Optimization . . . . . . . . . 98

6.2.2. Algorithm Initialization . . . . . . . . . . . . . . . . . . . . 99

6.3. Input Data: TanDEM-X Mosaics over Greenland . . . . . . . . . . . . 100

6.3.1. TanDEM-X Input Mosaics . . . . . . . . . . . . . . . . . . . . 100

6.3.2. Generation of the Ice Sheet Mask . . . . . . . . . . . . . . . 102

6.4. Classification Results . . . . . . . . . . . . . . . . . . . . . 103 
6.5. Snow Facies Interpretation and Further Considerations ． . . . . . . 108 6.5.1. Reference Snow Melt Data . . . . . . . . . . . . . . . . 108

6.5.2. In Situ Measurements along the EGIG Line . . . . . . . . . . . 110

6.5.3. Refined Classification of the Inner Snow Facies . . . . . . . . . 112

6.5.4. Statistical Analysis of the Derived Snow Facies . . . . . . . . . 113

6.5.5. Volume Decorrelation Dependency on the Height of Ambiguity 114

6.6. Applications . . . . . . . . . . . . . . . . . . . . 115

6.6.1. Backscatter Modeling using Modified Weighting . . . . . . . . 116

6.6.2. Estimation of the Penetration Depth . . . . . . . . . . . . . 120

7. Generation of Backscatter Maps 127

7.1. Generation of Backscatter Mosaics . . . . . . . . . . . . . . . . . . . . 127

7.2. Global Backscatter Map Final Structure . . . . . . . . . . . . . . . . . 134

7.3. Filling of Missing Values . . . . . . . . . . . . . . 135

$\begin{array}{ll}\text { 8. Conclusions and Future Work } & 138\end{array}$

$\begin{array}{ll}\text { Bibliography } & 143\end{array}$

$\begin{array}{ll}\text { Appendices } & 158\end{array}$

A. Backscatter Models Database 159 


\section{List of Symbols}

$\begin{array}{ll}a & \text { radar brightness quality weight } \\ A & \text { reference integration area } \\ A_{\beta} & \text { backscatter normalization area in slant range direction } \\ A_{\gamma} & \text { backscatter normalization area perpendicular to the slant range direction } \\ A_{\sigma} & \text { backscatter normalization area projected on ground } \\ \alpha & \text { local slope } \\ B & \text { baseline } \\ B_{\perp} & \text { normal baseline } \\ B_{\|} & \text {parallel baseline } \\ B_{\perp, c} & \text { critical baseline } \\ B_{\mathrm{rg}} & \text { pulse bandwidth } \\ \beta & \text { baseline orientation angle with respect to the local horizontal } \\ \beta^{0} & \text { radar brightness } \\ \beta_{u n W}^{0} & \text { mean radar brightness unweighted model } \\ \beta_{W}^{0} & \text { mean radar brightness weighted model } \\ \beta_{\text {sim }}^{0} & \text { simulated radar brightness image } \\ c & \text { number of output clusters } \\ \mathbf{C} & \text { noise covariance matrix for unweighted radar brightness measurements } \\ \mathbf{C}_{\mathrm{w}} & \text { noise covariance matrix for weighted radar brightness measurements } \\ D & \text { DEM in spatial distances coordinates } \\ d(\tau) & \text { complex chirp in range } \\ d_{\mathrm{az}} & \text { azimuth pulse repetition time } \\ d_{1 w} & \text { one-way penetration depth } \\ d_{2 w} & \text { two-way penetration depth } \\ \delta \beta^{0} & \text { radar brightness correction factor } \\ \Delta f_{\mathrm{R}} & \text { spectral shift } \\ \Delta h & \text { mean height difference between ICESat and TanDEM-X } \\ \Delta H & \text { difference between the mean estimated two-way penetration depth and the } \\ & \text { difference between ICESat and TanDEM-X } \\ & \end{array}$




\begin{tabular}{|c|c|}
\hline$\Delta R$ & range difference \\
\hline$\Delta \theta$ & incidence angle difference \\
\hline$e$ & Euler's number \\
\hline$\epsilon$ & regularization term \\
\hline$\varepsilon$ & complex dielectric constant \\
\hline$\varepsilon_{0}$ & permittivity of free space \\
\hline$\varepsilon^{\prime}$ & permittivity of the material relative to that of free space \\
\hline $\mathbf{E}$ & electric field intensity \\
\hline$E[\cdot]$ & mean value \\
\hline $\mathbf{E}(x, y, z)$ & vector phasor of the electric field \\
\hline $\mathbf{E}(x, y, z ; t)$ & instantaneous electric field at time $t$ \\
\hline$E_{\mathrm{x}}, E_{\mathrm{y}}, E_{\mathrm{z}}$ & Cartesian components of the electric field phasor \\
\hline$\eta$ & intrinsic impedance \\
\hline$f$ & frequency \\
\hline $\mathbf{F}$ & vector of observations belonging to a single snow facies \\
\hline $\bar{F}_{\mathrm{w}}$ & mean value of all pixels within the considered boxcar window \\
\hline$f_{\mathrm{D}}\left(t_{\mathrm{az}}\right)$ & Doppler frequency \\
\hline$\phi\left(t_{\mathrm{az}}\right)$ & azimuth phase \\
\hline$\phi_{\mathrm{a}}$ & squint angle \\
\hline$\phi_{o b j}$ & target's phase within a SAR image \\
\hline$\phi_{\text {int }}$ & interferometric phase \\
\hline$\phi_{f e}$ & flat Earth phase \\
\hline$\phi_{\text {topo }}$ & topographic phase \\
\hline$\phi_{\text {unw }}$ & unwrapped phase \\
\hline$\varphi$ & angle between the horizontal and the slant range direction \\
\hline$G$ & fitting Gaussian function \\
\hline$G_{\mathrm{t}}$ & transmitting antenna gain \\
\hline$G_{\mathrm{r}}$ & receiving antenna gain \\
\hline$\gamma$ & propagation constant \\
\hline$\gamma^{0}$ & backscattering coefficient per unit area perpendicular to the antenna beam \\
\hline$\gamma_{W}^{0}$ & mean $\gamma^{0}$ weighted model \\
\hline$\gamma_{\text {Tot }}$ & total interferometric coherence \\
\hline & correlation factor due to limited SNR \\
\hline Quant & correlation factor due to quantization \\
\hline
\end{tabular}




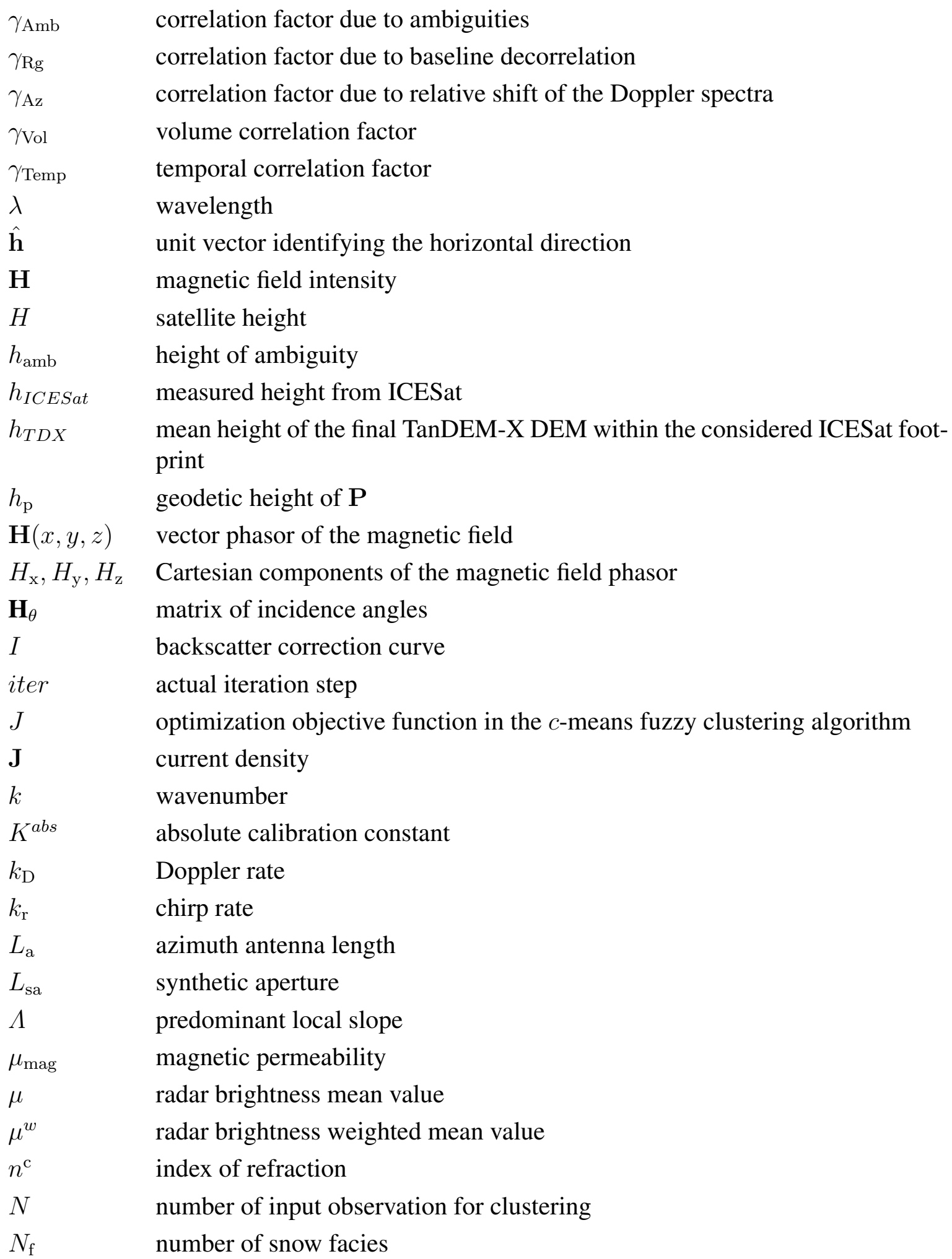




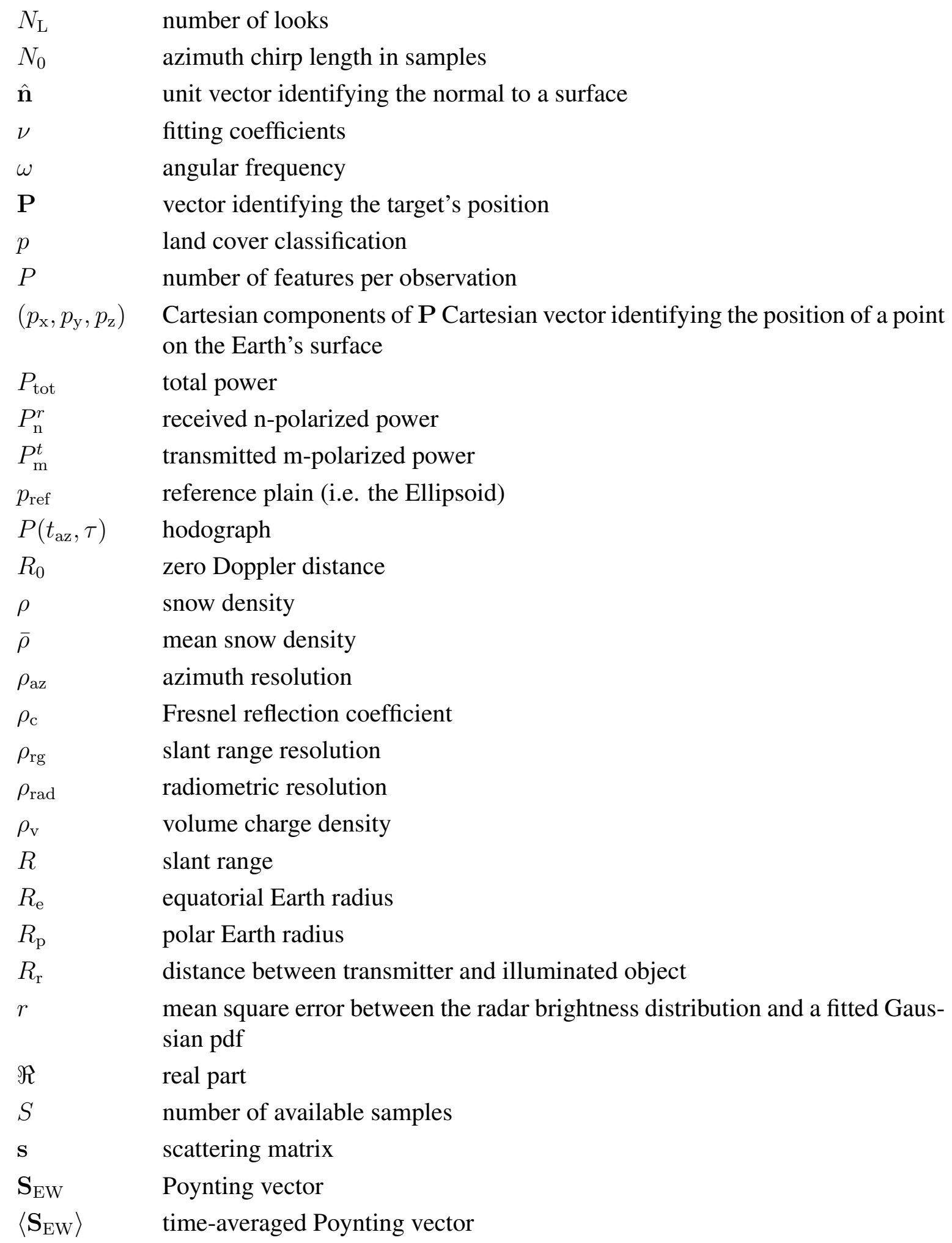


S vector identifying the sensor's position

$\left(s_{\mathrm{vv}}, s_{\mathrm{vh}}, \quad\right.$ polarized components of the object's scattering amplitude

$\left.s_{\mathrm{hv}}, s_{\mathrm{hh}}\right) \quad(\mathrm{h}=$ horizontal, $\mathrm{v}=$ vertical $)$

$S_{\mathrm{n}}^{s} \quad$ scattered power density at the location of the receiving antenna

$S_{\mathrm{m}}^{i} \quad$ power density illuminating the object

$s_{\mathrm{az}} \quad$ received signal in azimuth

$s_{\mathrm{r}} \quad$ received signal in range

$s_{\text {ac }} \quad$ azimuth compressed signal

$s_{\mathrm{cr}} \quad$ range compressed signal

$S 1, S 2 \quad$ InSAR satellites position

$\sigma^{0} \quad$ backscattering coefficient

$\sigma^{\text {ref }} \quad$ reference radar cross-section

$\sigma \quad$ standard deviation

$\sigma_{\mathrm{c}} \quad$ conductivity

$\sigma_{\mathrm{mn}} \quad$ mn-polarized radar cross section

$\sigma_{\mathrm{mn}}^{0}$

$\sigma_{W}^{0}$

$\sigma^{2}$

$\sigma^{2 w}$

$t$

$t_{\mathrm{az}}$

mn-polarized backscattering coefficient

mean $\sigma^{0}$ weighted model

radar brightness variance

radar brightness weighted variance

time

azimuth time

$T_{\mathrm{o}} \quad$ acquisition duration

$\tau \quad$ slant range time

$\tau_{\mathrm{c}} \quad$ Fresnel transmission coefficient

$\tau_{\text {rg }} \quad$ pulse duration

$\tau_{\mathrm{p}} \quad$ slant range of a point located on the Earth's surface

$\theta \quad$ incidence angle

$\theta_{\mathrm{az}} \quad$ angular resolution in azimuth

$\theta_{1} \quad$ local incidence angle

$\theta_{c} \quad$ critical angle

$\theta_{\mathrm{sa}} \quad$ azimuth angular resolution of the synthetic aperture

$\theta_{\mathrm{s}} \quad$ slave image incidence angle

$\theta_{\mathrm{m}} \quad$ master image incidence angle

$\theta_{\text {ref }} \quad$ reference incidence angle 


$\begin{array}{ll}u & \text { SAR image } \\ \mathbf{U} & \text { membership matrix } \\ (x, y, z) & \text { Cartesian coordinates system } \\ (\hat{x}, \hat{y}, \hat{z}) & \text { Cartesian unit coordinates vector } \\ \times & \text { vector product } \\ \boldsymbol{\xi} & \text { vector of polynomial coefficients to be estimated for unweighted radar bright- } \\ & \text { ness measurements } \\ \boldsymbol{\xi}^{w} & \text { vector of polynomial coefficients to be estimated for weighted radar bright- } \\ & \text { ness measurements } \\ v_{\mathrm{p}} & \text { wave phase velocity } \\ v_{\mathrm{s}} & \text { sensor speed } \\ \hat{\mathbf{v}} & \text { unit vector identifying the vertical direction } \\ V & \text { coefficient of variation } \\ v_{\text {lux }} & \text { speed of light } \\ \mathbf{w} & \text { zero mean white Gaussian noise for unweighted radar brightness measure- } \\ & \text { ments } \\ \hat{\mathbf{w}} & \text { vector of quality weights } \\ \mathbf{w}_{\mathrm{w}} & \text { zero mean white Gaussian noise for weighted radar brightness measurements } \\ w_{\mathrm{h}} & \text { boxcar half width } \\ \mathbf{Y} & \text { vector of input observation (backscatter and volume correlation coefficient) } \\ z & \text { for the classification of the Greenland Ice Sheet } \\ & \text { topographic height }\end{array}$




\section{List of Mathematical Operators}

$\begin{array}{ll}\Re & \text { real part } \\ \nabla & \text { gradient operator } \\ \nabla \cdot & \text { divergence operator } \\ \nabla \times & \text { curl operator } \\ \nabla^{2} & \text { Laplacian operator } \\ E[\cdot] & \text { mean value } \\ {[\cdot]^{\mathrm{T}}} & \text { matrix transposition } \\ (\cdot)^{*} & \text { complex conjugate } \\ \min (\cdot) & \text { minimum value } \\ \|\cdot\| & \text { norm of the argument }\end{array}$




\section{List of Acronyms}

$\begin{array}{ll}\text { ALOS } & \text { Advanced Land Observing Satellite } \\ \text { ALOS-2 } & \text { Advanced Land Observing Satellite 2 } \\ \text { ASAR } & \text { Advanced Synthetic Aperture Radar } \\ \text { ASC } & \text { Ascending Orbit Direction } \\ \text { BAQ } & \text { Block Adaptive Quantization } \\ \text { CEOS } & \text { Committee on Earth Observation Satellites } \\ \text { COSMO-SkyMed } & \text { Constellation of Small Satellites for Mediterranean basin Obser- } \\ \text { CoSSC } & \text { vation } \\ \text { DEM } & \text { Coregistered Single look Slant range Complex } \\ \text { DESC } & \text { Digital Elevation Model } \\ \text { DLR } & \text { Descending Orbit Direction } \\ \text { DN } & \text { Deutsches Zentrum für Luft- und Raumfahrt } \\ \text { EM } & \text { Digital Number } \\ \text { ENVISAT } & \text { Electromagnetic Wave } \\ \text { ERS-1 } & \text { Environmental Satellite } \\ \text { ERS-2 } & \text { European Remote-sensing Satellite 1 } \\ \text { ESA } & \text { European Remote-sensing Satellite 2 } \\ \text { GLOBCOVER } & \text { European Space Agency } \\ \text { GTOPO30 } & \text { Global Land Cover Map } \\ \text { HH } & \text { Global 30 Arc-Second Elevation Model } \\ \text { HUTSCAT } & \text { Horizontal polarization in transmission, Horizontal polarization } \\ \text { HV } & \text { in reception } \\ \text { ICESat } & \text { Helsinki University of Technology Scatterometer } \\ \text { InSAR } & \text { Horizontal polarization in transmission, Vertical polarization in } \\ \text { ITP } & \text { reception } \\ \text { LHC } & \text { Ice, Cloud, and land Elevation Satellite } \\ & \text { SAR interferometry } \\ \text { Integrated TanDEM-X Processor } \\ \text { Left-Hand Circular } \\ \end{array}$


LHE

JAXA

JERS-1

KOMPSAT-5

NASA

NESZ

NSCAT

PALSAR

pdf

PRF

RADARSAT

rawDEM

RCS

RHC

RHE

RMSE

ROI

SAR

SIR-A

SIR-B

SLAR

SRTM

SNR

TanDEM-X

TEM

TerraSAR-X

TSX

TDX

USGS

VV

$\mathrm{VH}$

WGS84
Left-hand Elliptical

Japan Aerospace Exploration Agency

Japanese Earth Resources Satellite 1

Korea Multi Purpose Satellite 5

National Aeronautics and Space Administration

Noise Equivalent Sigma Zero

NASA Scatterometer

Phased Array type L-band Synthetic Aperture Radar

probability density function

Pulse Repetition Frequency

Radar Satellite

TanDEM-X geocoded and roughly calibrated DEM

Radar Cross Section

Right-Hand Circular

Right-Hand Elliptical

Root Mean Square Error

Region of Interest

Synthetic Aperture Radar

Shuttle Imaging Radar A

Shuttle Imaging Radar B

Side Looking Airborne Radar

Shuttle Radar Topography Mission

Signal-to-Noise Ratio

TerraSAR-X add-on for Digital Elevation Measurements

Transverse Electromagnetic Wave

Terra (Earth) Synthetic Aperture Radar X-Band

TerraSAR-X Satellite

TanDEM-X Satellite

United States Geological Survey

Vertical polarization in transmission, Vertical polarization in reception

Vertical polarization in transmission, Horizontal polarization in reception

World Geodetic System 1984 


\section{Introduction}

\subsection{Radar in Remote Sensing}

Remote sensing identifies the applied science which allows for the retrieval of information about a distant object, without having any physical interaction with it. In the last decades it has become one of the most advanced techniques for observing our planet and studying its dynamic processes. The urgency for a global monitoring of climate changes and their consequences has pushed for the development of airborne and spaceborne missions, providing a unique data base for scientific applications.

Remote sensing sensors can be divided into two categories: passive and active. Passive sensors capture the reflected solar radiation or the thermal radiation emitted from the Earth's surface itself. On the other hand, active sensors, such as radar, transmit their own signal and receive that portion of the signal which is redirected back from the ground towards the sensor. They have the advantage of being able to operate without the presence of sun light and they are not significantly effected by clouds or precipitation.

Radar systems can be classified as monostatic, if the transmitting antenna coincides with the receiving one, or bistatic (or multistatic, depending on the number of receiving antennas) if different antennas are used for transmitting and receiving. They operate in the domain of the microwaves (from $300 \mathrm{MHz}$ to $300 \mathrm{GHz}$ ) and their ability to penetrate clouds, precipitation, or land surface cover typically depends on the carrier frequency: the longer the wavelength, the higher the penetration capability. Typical carrier frequencies used for remote sensing purposes are summarized in Table 1.1.

Radar sensors for remote sensing applications can be divided into three classes: scatterometers, altimeters, and imaging radars.

- Scatterometers are radar devices designed to determine the backscatter level of the illuminated area [1], defined as the portion of the transmitted electromagnetic signal that the target redirects back towards the radar antenna. Backscatter data from scatterometers are applied to the study of vegetation, polar ice, soil moisture, and ocean current tracking.

- Radar altimeters are used to measure the altitude above the terrain beneath the sensor platform by measuring the time delay that occurs between the transmission and reception times of the reflected radio wave. 
Table 1.1.: Summary of typical carrier frequencies for remote sensing radar systems.

\begin{tabular}{lcc}
\hline \hline \multicolumn{3}{c}{ Radar Frequencies } \\
\hline Denomination & Frequency & Wavelength \\
\hline & {$[\mathbf{G H z}]$} & {$[\mathbf{c m}]$} \\
\hline$P$ & $0.23-1$ & $30-130$ \\
\hline$L$ & $1-2$ & $15-30$ \\
\hline$S$ & $2-4$ & $7.5-15$ \\
\hline$C$ & $4-8$ & $3.75-7.5$ \\
\hline$X$ & $8-12$ & $2.5-3.75$ \\
\hline$K_{u}$ & $12-18$ & $1.67-2.5$ \\
\hline$K$ & $18-27$ & $1.11-1.67$ \\
\hline$K_{a}$ & $27-40$ & $0.75-1.11$ \\
\hline$V$ & $40-75$ & $0.4-0.75$ \\
\hline$W$ & $75-110$ & $0.27-0.4$ \\
\hline
\end{tabular}

- Imaging radars are able to map the returning electromagnetic waves from onground objects onto a two-dimensional plane. The intensity of the backscattered signals can be properly composed to obtain an optical-like image of the illuminated ground: the higher the received signal is, the brighter the corresponding pixel inside the final image will be. Moreover, the information about the phase of the reflected wave is available as well, containing information on the distance of the illuminated objects from the sensor. Modern imaging radar techniques are based on the Synthetic Aperture Radar (SAR) principle, which exploits the motion of the radar antenna over a defined ground region, in order to obtain a finer resolution.

Given their characteristics, SAR images are suitable not only for simple imaging purposes, but for a variety of different scientific applications as well, among which are:

- SAR interferometry: by exploiting the phase difference between two or more SAR images acquired at different sensor positions, it is possible to generate elevation maps of the surface [2]. If a series of images subsequently acquired is considered, it is possible to detect the changes in time of the position of the illuminated targets on ground, allowing to monitor subsidence and deformation phenomena (differential interferometry) [3].

- SAR tomography: SAR interferometry has the limitation of retrieving the simple phase center of all the targets on ground within a resolution cell, which does not always coincide with the terrain topograpy (e.g. in the presence of vegetation or urban areas). SAR tomography was born in order to fill this gap, by estimating the 
vertical distribution of the scatterers, by means of repeat-pass acquisitions over the same area from slightly different positions [4].

- SAR polarimetry: it is based on the combination of SAR images acquired over the same ground area with different wave polarizations. In this way, different backscattering mechanisms can be characterized and associated to several physical processes [5], [6].

\subsection{A Historical Overview of Spaceborne SAR}

The birth of radar dates back to the beginning of the twentieth century. In 1904, building on Heinrich Hertz's discovery that electromagnetic waves were reflected from metallic surfaces [7], Christian Hülsmeyer invented the so called Telemobiloscope, able to detect the presence of distant objects using electromagnetic waves [8]. However, the working principle of radar can even be found in nature. Bats, for example, utilize a natural SONAR (SOund Navigation And Ranging), being able to locate animals by emitting calls out in the environment and listening to those calls which return from objects nearby. The term RADAR was coined in 1939 by the Unites States Navy, as the acronym of RAdio Detection And Ranging, becoming later on a common noun. During World War II, radar started to be used for object detection purposes, and later on for civilian applications as well, such as traffic and weather monitoring.

After the second World War, the SLAR (Side Looking Airborne Radar) was invented, aiming at imaging ground areas from airborne platforms. Flying along a defined path (along track dimension), it was able to acquire images by pointing the antenna beam perpendicularly to the flight direction (across track dimension). Its resolution, defined as the ability to distinguish between two targets on ground, depends on the transmitted pulse bandwidth in the across track direction, and on the beamwidth of the radar antenna in the along track direction.

At the beginning of the 1950s the need for higher-resolution images led to the development of Synthetic Aperture Radar (SAR). In 1951, Carl A. Wiley observed that a finer resolution in the along track dimension can be obtained by exploiting the motion of the radar antenna over a target region [9]. The principle resides in synthesizing an antenna of large dimensions (on the order of kilometers) in the along track dimension, by properly combining the information coming from each target on ground, obtained at different positions of the radar sensor. Since then, SAR has been widely recognized as a paramount mean for remote sensing applications, and an increasing number of airborne and spaceborne missions has been developed throughout the years, significantly improving the resolution and quality of the delivered images.

NASA Jet Propulsion Laboratory's Seasat was the first spaceborne SAR satellite (Fig. 


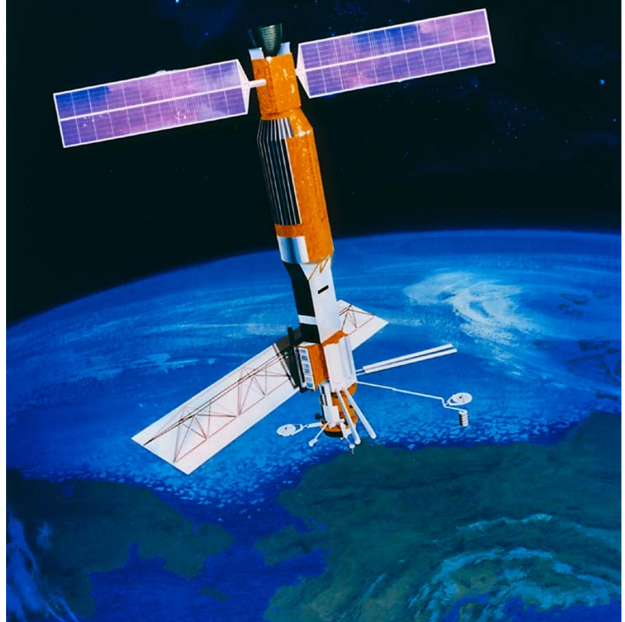

(a)

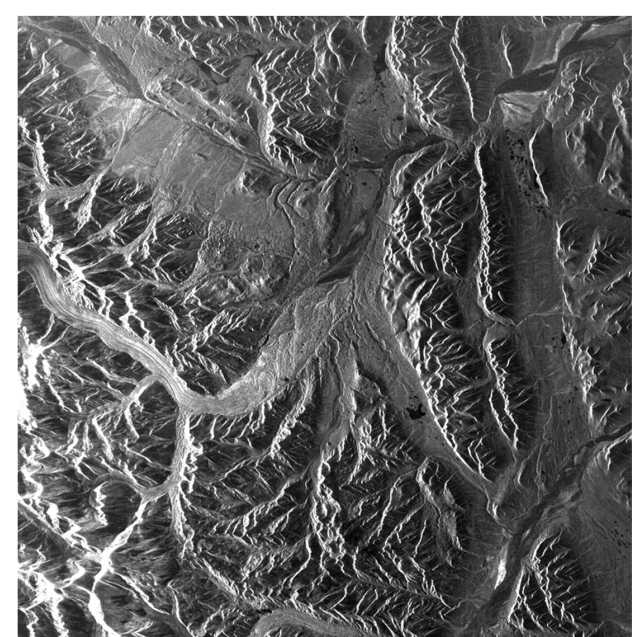

(b)

Figure 1.1.: (a) NASA's Seasat satellite, launched in 1978. (b) Seasat SAR image over the Teepee Park, Yukon, Canada. Acquired on July $21^{\text {st }}, 1978$, by NASA.

1.1) [10]. Launched in 1978, it was mounted with an L-band radar antenna, designed to monitor oceanographic phenomena on a global scale, such as sea surface winds and temperature, wave heights, and sea ice features. Later on, a series of L-band SAR sensors were mounted on-board the Space Shuttle, leading to the Shuttle Imaging Radar missions SIR-A (1981) [11], [12] and SIR-B (1984) [13].

The Japanese Space Agency (JAXA) further developed L-band spaceborne SAR sensors, launching JERS-1 in 1992, ALOS in 2006, and ALOS-2 in 2014 [14], [15].

The first remote sensing satellite developed by the European Space Angency (ESA) was ERS-1 [16]. Launched in 1991, it was equipped with a C-band SAR antenna and operated until 2000. The continuity of acquired data was assured first by its follow-up twin satellite ERS-2, launched in 1995, and then by ENVISAT, launched in 2002 and in operation until 2012 [17] [18]. The ENVISAT satellite mounted nine different instruments for the observation of the Earth, among which the ASAR C-band active antenna array, allowed to electronically steer the antenna beam in both transmitting and receiving directions. In 2014 and 2016, ESA launched Sentinel-1A and Sentinel-1B, respectively, as part of the Copernicus Program [19]. These two satellites are equipped with a SAR antenna operating at $\mathrm{C}$ band, and provide continuity of data after ENVISAT, with enhanced capabilities in terms of revisit time and coverage.

The Canadian Space Agency launched RADARSAT-1 and RADARSAT-2 in 1995 and 2007, respectively: two C-band SAR satellites, whose orbit is optimized for regularly observing the Arctic up to the pole, at the disadvantage of loosing the illumination of the central areas of Antarctica [20], [21]. 
Another milestone in spaceborne SAR is represented by the SIR-C/X-SAR missions, developed in a joint venture between NASA JPL and a European consortium of the German Aerospace Center (DLR) and the Italian Space Agency (ASI) [22]. The instrument, with multi-bandwidth capabilities (L, C, and X bands), was flown on two Space Shuttle flights in April and October 1994, opening the way for the development of the Shuttle Radar Topography Mission (SRTM) in 2000 [23]. Both NASA and DLR took part in that mission, whose objective was the generation of an Earth Digital Elevation Model (DEM) on a near global scale, from $56^{\circ} \mathrm{S}$ to $60^{\circ} \mathrm{N}$ latitudes.

In June 2007, TerraSAR-X was launched: an imaging radar Earth observation satellite, developed under a public-private-partnership between DLR and Airbus Defence \& Space. The satellite operates at $\mathrm{X}$ band and is able to acquire high-quality SAR images

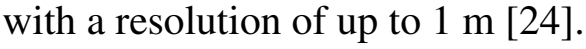

A twin satellite, TanDEM-X, was then launched in 2010, officially starting, together with TerraSAR-X, the bistatic TanDEM-X mission. Flying in a close-orbit configuration, both satellites act as single-pass interferometer, allowing for the acquisition of high-resolution interferograms [25]. The generation of a global DEM, completed in 2016, was the main objective of the TanDEM-X mission and the generation of an additional change-DEM layer is foreseen for 2020.

Other on-going spaceborne SAR missions are the Italian X-band constellation of four satellites COSMO-SkyMed [26], the first military-civil system for Earth observation, and the Korean KOMPSAT-5 [27], an X-band SAR mission launched in 2013.

\subsection{Motivation of the Work}

Radar backscatter represents a fundamental quantity in radar measurements. Its properties change depending on several factors, such as sensor parameters (e.g. frequency and polarization), soil conditions, and surface roughness. Moreover, it is influenced by atmospheric conditions, on-ground vegetation, topographic characteristics of the illuminated ground area, and acquisition geometry.

High accuracy in commanding, processing and system performance is required in order to provide high quality SAR images. The accurate knowledge of backscatter represents a valuable input for an optimized operation of the whole SAR system.

As an example, one can take into account the TerraSAR-X and TanDEM-X satellites, where no automatic adaptation of the commanding radar parameters is performed onboard during a data take. Acquisitions are commanded with pre-defined receiver gain setting and no automatic gain control is performed. For known backscatter characteristics of a requested SAR scene, the receiver gain can be suitably adapted to mitigate clipping or signal saturation [24].

Moreover, during the design phase of future SAR missions, an acurate knowledge of 
backscatter is necessary for the prediction of SAR performance, and therefore for the optimization of the design strategy. An example is given by the design of the future Tandem-L mission, which will make use of innovative digital beamforming techniques to achieve high-resolution wide swath imaging [28], [29].

Backscatter information is widely used for a number of scientific applications as well. For example, backscatter levels can be related to biomass using polarimetry [30], or serve as input for retrieving snow mass from radar images [31].

With the availability of X-band SAR data for scientific purposes, the investigation of $\mathrm{X}$-band backscatter from spaceborne platforms has become a highly interesting research topic in the last years. Furthermore, the global coverage provided by the TanDEM-X mission, together with the availability of a fine-scale land cover classification, allows for the generation an up-to-date data set of radar backscatter models at X band, opening the door for the generation of high resolution X-band backscatter maps on a global scale, which represents a highly interesting topic for both the scientific community and the design of future SAR missions.

The main goal of this work is to exploit the global TanDEM-X data set to statistically model radar backscatter at $\mathrm{X}$ band, considering different acquisition parameters and land cover types, and to provide the remote sensing community with an up-to-date data base of models, describing the Earth's reflectivity at X band.

The backscatter dependency on season and geographic location has been investigated as well and a proper selection of the input observations allows to derive specific models, which locally increase the final accuracy.

Moreover, particular attention has been focused on the Greenland Ice Sheet, which represents a highly interesting topic for the scientific community. In fact, melt phenomena have strongly increased in the last years, leading to modifications in the characteristics of the snow pack. A better knowledge of the Ice Sheet can substantially contribute to a better understanding of the arctic and its response to climate change. The unique interferometric signature of the bistatic TanDEM-X system has allowed for the development of a method to characterize different snow facies and subsequently refine the derived backscatter models, depending on the characteristics of the snow pack.

Finally, the availability of the global TanDEM-X SAR data set, together with the derived data base of backscatter models, allows for the derivation of a global backscatter map at $\mathrm{X}$ band, a key-quantity for the design of future SAR missions.

\subsection{Thesis Structure}

The thesis is structured as follows: 
- Basic concepts on electromagnetic waves, radar backscatter, SAR image formation, and SAR interferometry are summarized in chapters 2 and 3. This theoretical part is not meant as a comprehensive compendium on the proposed subjects, but rather as a summary of all necessary concepts for understanding the developed work.

- An overview of the TanDEM-X mission is presented in chapter 4, together with the description of the global data set of derived quicklook images. Such data serves as input for the application, testing, and validation of the developed algorithms.

- The newly developed backscatter modeling algorithm is presented in chapter 5, together with a discussion on the sources of error and on the proposed models verification approach. Moreover, the impact of backscatter seasonal changes within the modeling process is addressed as well and a possible solution is proposed. The attention is then focused on rain forests, which represent a widely used target for SAR calibration purposes. Here, an analysis of backscatter behavior depending on acquisition daytime and geometry is presented and discussed.

- Chapter 6 is dedicated to the Greenland Ice Sheet. The presence of different kinds of snow cover and the lack of an appropriate ground classification map led to the development of the proposed algorithm for the identification of different snow facies. Based on the derived two-dimensional snow facies map, ad-hoc backscatter models are derived for each snow facies, separately, and the radar wave penetration depth into the ice sheet is estimated as well.

- Chapter 7 presents a possible application of a global data base of backscatter models, consisting in the generation of large-scale backscatter maps. Here, the developed algorithm for the equalization of independent input backscatter data to a common reference incidence angle is presented, together with their mosaicking on a common output grid. An iterative algorithm is finally implemented to fill remaining gaps due to missing input data.

- Finally, conclusions and future work are summarized in chapter 8, while a data base of polynomial backscatter models and statistical values, obtained from the global TanDEM-X mission data, is provided in appendix A.

\subsection{Original Contribution of the Thesis}

This thesis is the result of the work carried out at the Microwaves and Radar Institute (HR) of the German Aerospace Center (DLR), Germany. Its original contribution comprises: 
- The extraction and processing of the entire global data set of TanDEM-X quicklook images (lower resolution images derived by averaging full resolution ones), together with the derivation of additional quantities, such as the signal-to-noise ratio (SNR) or the terrain predominant slope, which are necessary for the development of the presented algorithms and analysis.

- The development of a new algorithm for modeling radar backscatter. The unique amount of data made available by the TanDEM-X mission has provided the motivation to model backscatter by concentrating on the statistics provided by the SAR data itself, rather than building a theoretical model, based on the physical principles of radar backscattering mechanisms.

- The generation of an up-to-date data base of backscatter models for X band, covering different kinds of land cover at a global scale.

- The seasonal and geographic dependency of the backscatter has been analyzed as well and backscatter models have been separately derived for different seasons. In particular, the analysis of such a dependency has been focused on the tropical rain forest, which, given its isotropic characteristics, is commonly used as a reference distributed target for sensor calibration purposes.

- A dedicated analysis of the Greenland Ice Sheet, aimed at locating different snow facies, as seen by TanDEM-X. A classification approach has been developed taking into account the interferometric signature of TanDEM-X data.

The derived snow facies are used for two main purposes:

- To provide a set of backscatter models, depending of the characteristics of the snow pack,

- To estimate the two-way X-band penetration depth into the ice sheet.

- Finally, an algorithm for the generation of backscatter maps has been developed and represents a possible application of the derived backscatter models data base.

Moreover, the research work presented in this thesis can also be seen as an ignition for the development of the global TanDEM-X Forest/Non-Forest Map product, derived from the global data set of TanDEM-X bistatic acquisitions [32], and of the related ongoing activities at the institute, aimed at developing novel multi-sensor approaches for monitoring deforestation [33].

It is also worth mentioning that the derived snow facies map is the first information product on a continental scale, generated by an interferometric spaceborne SAR mission beyond the standard digital elevation model (DEM). 


\section{Theoretical Background}

\subsection{Electromagnetic Waves}

To fully understand radar backscattering mechanisms, it is important to recall the Electromagnetic (EM) Wave theory basics and the way in which such waves interact with materials. An exhaustive compendium of the subject can be found in [34] and [1]. This section provides a short overview of EM waves, focusing on EM plane waves and some of their properties, which are useful for the understanding of the present work. The content of this section is confined to lossless media, where waves do not suffer from any attenuation. A more detailed description of propagation in lossy media can be found in [35] and [1].

\subsubsection{Electromagnetic Plane Waves}

An EM wave is the result of a time-varying electric field which induces a magnetic field or, vice versa, of a time-varying magnetic field which induces an electric one. Waves can propagate both in lossless mediums, without attenuation, or in lossy ones, where part of the wave's energy is dissipated into heat. Materials in general are characterized by four constitutive parameters [1]:

- the conductivity $\sigma_{\mathrm{c}}$,

- the volume charge density $\rho_{\mathrm{v}}$,

- the magnetic permeability $\mu_{\mathrm{mag}}$,

- the electrical permittivity $\varepsilon^{\prime} \varepsilon_{0}$, where $\varepsilon_{0}$ is the permittivity of free space and $\varepsilon^{\prime}$ is the permittivity of the material relative to that of free space.

If a wave is generated by a punctiform or localized source, it expands in all directions with the same velocity, leading to the generation of a spherical wave. If the wave's front is observed at a very large distance from the source (in the so-called far field region), it appears approximately planar, with identical properties all over the plane tangent to the wavefront. Such waves are called plane waves and they can be described in a Cartesian coordinate system $(x, y, z)$.

The way in which electric and magnetic fields are generated and altered by each other and by currents and charges is described by Maxwell's differential equations, which, in a homogeneous and isotropic medium, are given by: 


$$
\begin{array}{rlr}
\nabla \cdot \mathbf{E} & =\frac{\rho_{v}}{\varepsilon^{\prime} \varepsilon_{0}} & \text { (Gauss's law) } \\
\nabla \times \mathbf{E} & =-\mu_{\mathrm{mag}} \frac{\partial \mathbf{H}}{\partial t} & \text { (Faraday's law) } \\
\nabla \cdot \mathbf{H} & =0 & \text { (Gauss's law for magnetism) }, \\
\nabla \times \mathbf{H} & =\mathbf{J}+\varepsilon^{\prime} \varepsilon_{0} \frac{\partial \mathbf{E}}{\partial t} & \text { (Ampère's law) },
\end{array}
$$

where $\mathbf{E}$ is the electric field intensity, $\mathbf{H}$ the magnetic field intensity, and $\mathbf{J}$ the current density flowing through the medium.

If the time variation of the electric and magnetic fields $(\mathbf{E}$ and $\mathbf{H})$ is sinusoidal with angular frequency $\omega, \mathbf{E}$ and $\mathbf{H}$ can be represented with vector phasors which depend on $(x, y, z)$ only. In this case, the vector phasor of the electric field $\mathbf{E}(x, y, z)$ is related to the instantaneous electric field at the time $t, \mathbf{E}(x, y, z ; t)$, by:

$$
\mathbf{E}(x, y, z ; t)=\Re\left[\mathbf{E}(x, y, z) e^{j \omega t}\right],
$$

where $j=\sqrt{-1}$.

Differentiation in the time domain corresponds to a multiplication by $j \omega$ in the phasor domain. Maxwell's equations in the phasor domain therefore become:

$$
\begin{aligned}
\nabla \cdot \mathbf{E} & =0, \\
\nabla \times \mathbf{E} & =-j \omega \mu_{\operatorname{mag}} \mathbf{H}, \\
\nabla \cdot \mathbf{H} & =0, \\
\nabla \times \mathbf{H} & =j \omega \varepsilon \varepsilon_{0} \mathbf{E},
\end{aligned}
$$

where $\varepsilon$ is the complex dielectric constant, defined as [1]:

$$
\varepsilon=\varepsilon^{\prime}-j \frac{\sigma_{\mathrm{c}}}{\omega \varepsilon_{0}} .
$$

The explicit solutions for $\mathbf{E}$ and $\mathbf{H}$ can be derived from Maxwell's equations as presented in [34], obtaining the homogeneous wave equation for $\mathbf{E}$ and $\mathbf{H}$ as:

$$
\begin{aligned}
& \nabla^{2} \mathbf{E}+\gamma^{2} \mathbf{E}=0, \\
& \nabla^{2} \mathbf{H}+\gamma^{2} \mathbf{H}=0,
\end{aligned}
$$

where $\gamma$ represents the propagation constant and is defined as:

$$
\gamma^{2}=\omega^{2} \mu_{\mathrm{mag}} \varepsilon \varepsilon_{0}
$$


Since both (2.11) and (2.12) are of the same form, the solution of the wave equation is now derived for $\mathbf{E}$ only. The same considerations stand for $\mathbf{H}$ as well.

By considering a lossless medium, where $\varepsilon=\varepsilon^{\prime}$, and by defining the wavenumber $k$ as:

$$
k=\omega \sqrt{\mu_{\mathrm{mag}} \varepsilon^{\prime} \varepsilon_{0}},
$$

(2.11) can be written as:

$$
\nabla^{2} \mathbf{E}+k^{2} \mathbf{E}=0
$$

In Cartesian coordinates the electric field phasor can be decomposed as:

$$
\mathbf{E}=\hat{\mathbf{x}} E_{\mathrm{x}}+\hat{\mathbf{y}} E_{\mathrm{y}}+\hat{\mathbf{z}} E_{\mathrm{z}}
$$

where $(\hat{\mathbf{x}}, \hat{\mathbf{y}}, \hat{\mathbf{z}})$ is the Cartesian unit vector, and the Laplacian of $\mathbf{E}$ is given by:

$$
\nabla^{2} \mathbf{E}=\left(\frac{\partial^{2}}{\partial x^{2}}+\frac{\partial^{2}}{\partial y^{2}}+\frac{\partial^{2}}{\partial z^{2}}\right) \mathbf{E}
$$

By substituting (2.17) into (2.11), the following relationship is obtained for the $E_{\mathrm{x}}$ component:

$$
\left(\frac{\partial^{2}}{\partial x^{2}}+\frac{\partial^{2}}{\partial y^{2}}+\frac{\partial^{2}}{\partial z^{2}}+k^{2}\right) E_{x}=0 .
$$

Analogous expressions are valid for $E_{\mathrm{y}}$ and $E_{\mathrm{z}}$, which means that, if an infinite plane is considered, both the electric and magnetic fields components belonging to it are characterized by uniform properties and their derivatives are therefore equal to zero. Assuming such a plane to be the $(x, y)$ one, $(2.18)$ becomes:

$$
\frac{\partial^{2} E_{\mathrm{x}}}{\partial z^{2}}+k^{2} E_{\mathrm{x}}=0
$$

The same applies to $E_{\mathrm{y}}, H_{\mathrm{x}}$, and $H_{\mathrm{y}}$. For the remaining components it is valid that $E_{z}=H_{z}=0$, leading to the conclusion that the direction of propagation of a plane wave is characterized by the absence of electric and magnetic field components. The general solution of the ordinary differential equation in (2.19) can be derived by applying the boundary conditions, as presented in [1]. For the phasor $E_{\mathrm{x}}$ it can be obtained that:

$$
E_{\mathrm{x}}(z)=E_{\mathrm{x} 0}^{+} e^{-j k z}+E_{\mathrm{x} 0}^{-} e^{j k z},
$$

where $E_{\mathrm{x} 0}^{+}$and $E_{\mathrm{x} 0}^{-}$are two constants, whose value can be determined by applying the boundary conditions. The term $e^{-j k z}$ identifies a wave travelling in the $+z$ direction, while the term $e^{j k z}$ represents a wave travelling in the $-z$ one. The same kind of solution can be derived for the other $\mathbf{E}$ and $\mathbf{H}$ components. The electric and magnetic field components are perpendicular to each other and to the direction of propagation of the 


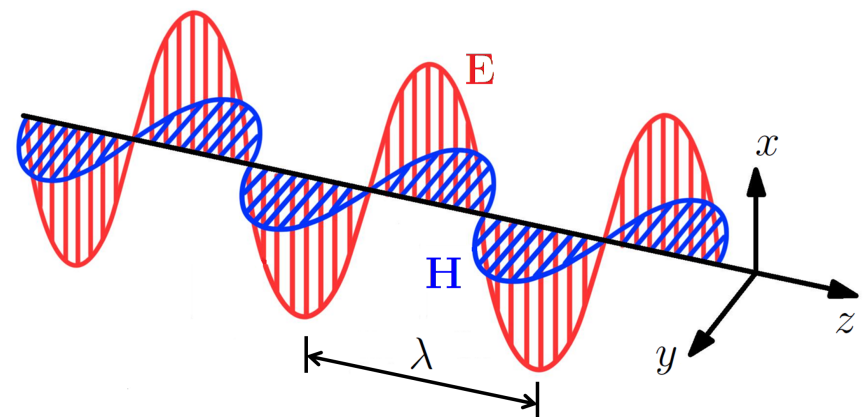

Figure 2.1.: Propagation of a TEM wave, decomposed into its electric (E) and magnetic (H) field components, which are perpendicular to the propagation's direction along the $z$ axis. $\lambda$ represents the wavelength.

wave, identifying a so called transverse electromagnetic (TEM) wave, whose representation is shown in Fig. 2.1. The direction of propagation of such a wave is defined by the cross product $\mathbf{E} \times \mathbf{H}$, being the latter oriented accordingly and parallel to the $z$ axis. Assuming to have $\mathbf{E}$ components along $x$ only $\left(E_{y}=0\right)$ and travelling in the $+z$ direction only, (2.20) becomes:

$$
\mathbf{E}(z)=\hat{\mathbf{x}} E_{\mathrm{x} 0}^{+} e^{-j k z},
$$

and, by applying (2.7), the associated magnetic field is:

$$
\mathbf{H}(z)=\hat{\mathbf{y}} H_{\mathrm{y} 0}^{+} e^{-j k z},
$$

where:

$$
H_{\mathrm{y} 0}^{+}=\frac{k}{\omega \mu_{\mathrm{mag}}} E_{\mathrm{x} 0}^{+} .
$$

For a lossless medium, the intrinsic impedance can now be defined as the ratio between the magnitude of the electric and magnetic fields as:

$$
\eta=\frac{E_{x 0}^{+}}{H_{y 0}^{+}}=\frac{\omega \mu_{\mathrm{mag}}}{k}=\sqrt{\frac{\mu_{\mathrm{mag}}}{\varepsilon^{\prime} \varepsilon_{0}}} .
$$

The $\mathbf{E}$ and $\mathbf{H}$ components on the $z$ plane, perpendicular to the propagation direction, show the same dependency on time $t$, by mean that they both reach their maximum or minimum amplitudes at the same time. This is a typical characteristic of waves in lossless media and both $\mathbf{E}$ and $\mathbf{H}$ are said to be in phase. Hence, the phase velocity of the wave can now be defined as: 


$$
v_{\mathrm{p}}=\frac{\omega}{k}=\frac{1}{\sqrt{\mu_{\mathrm{mag}} \varepsilon^{\prime} \varepsilon_{0}}}
$$

and the wavelength as:

$$
\lambda=\frac{2 \pi}{k}=\frac{v_{\mathrm{p}}}{f}
$$

where $f=\omega / 2 \pi$ is the frequency.

\subsubsection{Electromagnetic Power Density}

The energy flow carried by an electromagnetic wave per unit area $\mathbf{S}_{\mathrm{EW}}(t)$ is described by the Poynting vector:

$$
\mathbf{S}_{\mathrm{EW}}(t)=\mathbf{E}(t) \times \mathbf{H}(t) .
$$

$\mathbf{S}_{\mathrm{EW}}(t)$ is perpendicular to $\mathbf{E}(t)$ and $\mathbf{H}(t)$, and oriented accordingly to the wave's propagation direction. From (2.27) the time-averaged Poynting vector per unit time $T$ for EM planar waves is derived as [34]:

$$
\left\langle\mathbf{S}_{\mathrm{EW}}\right\rangle=\frac{1}{T} \int_{0}^{T} \mathbf{S}_{\mathrm{EW}}(t) d t=\frac{1}{2} \Re\left[\mathbf{E} \times \mathbf{H}^{*}\right] .
$$

By assuming that the wave flows through an aperture of area $A$, the total power $P_{\text {tot }}$ that is intercepted by the aperture is derived by integrating $\left\langle\mathbf{S}_{\mathrm{EW}}\right\rangle$ over the surface $A$ :

$$
P_{\mathrm{tot}}=\int_{A}\left\langle\mathbf{S}_{\mathrm{EW}}\right\rangle \cdot \hat{\mathbf{n}} d A,
$$

where $\hat{\mathbf{n}}$ represents the normal to the surface.

\subsubsection{Wave Polarization}

Polarization is a characteristic of electromagnetic waves and indicates the direction of oscillation of the electric field during the wave's propagation in space and time. The magnetic field is consequently polarized in the orthogonal direction with respect to the electric field and to the propagation's direction. If the direction of oscillation of the electric field randomly changes, such wave is said to be unpolarized. For plane waves, three types of polarization can be distinguished:

- Linear polarization: the electric field oscillates in one direction only, describing a linear segment in time on the plain in which it resides. Moreover, the plane 


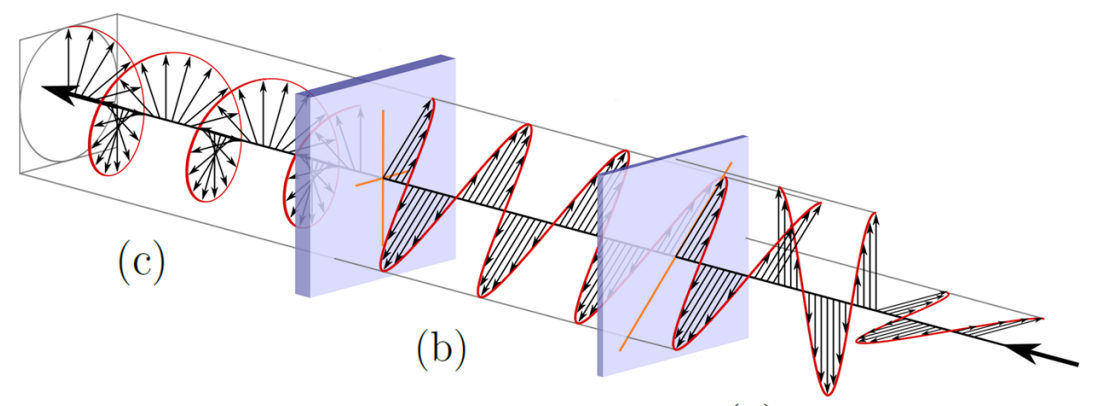

(a)

Figure 2.2.: Polarization of an electromagnetic wave. The electric field $\mathbf{E}$ is depicted for: (a) unpolarized wave, (b) linearly polarized wave, (c) left-hand circularly (LHC) polarized wave [36].

of incidence is defined as the plane to which the direction of propagation of the incident wave and the normal to the boundary belong to. Linearly polarized waves can be further classified as horizontal, if the electrical field is perpendicular to such a plane, or vertical, if the electrical field is parallel to it.

- Circular Polarization: the electric field describes a circle in time on the plain in which it resides. It can be distinguished between left-hand circular polarization (LHC), if the electric field describes in time a clockwise circle, and right-hand circular polarization (RHC), if the electric field describes in time a counterclockwise circle.

- Elliptical Polarization: the electric field describes an ellipse in time in the plane in which it resides. Also in this case it is possible to distinguish between left-hand (LHE) and right-hand elliptical (RHE) polarization.

A summary of different polarization is shown in Fig. 2.2.

\subsubsection{Wave Reflection and Transmission at the Interface}

Consider now two different homogeneous and lossless dielectric media, with permeability $\mu_{\text {mag, } 0}$ and relative permittivity $\varepsilon_{1}^{\prime}$ and $\varepsilon_{2}^{\prime}$, respectively. The considered media are separated by a planar interface located at $z=0$, as presented in Fig. 2.3 (a). Assume to have a TEM wave, which propagates within medium 1 in the direction perpendicular to the $z=0$ plain, towards the interface with medium 2. The incident electric and magnetic fields are identified as $\mathbf{E}^{i}$ and $\mathbf{H}^{i}$. At the moment of the interaction between the incident wave and the interface $(z=0)$, a portion of the incident electric and magnetic fields $\left(E_{0}^{i}\right.$ and $\left.H_{0}^{i}\right)$ is reflected back into medium $1\left(E_{0}^{r}\right.$ and $\left.H_{0}^{r}\right)$, while the rest is transmitted into 


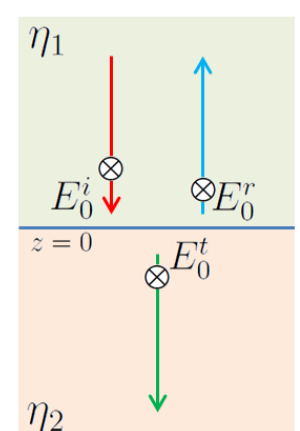

(a)

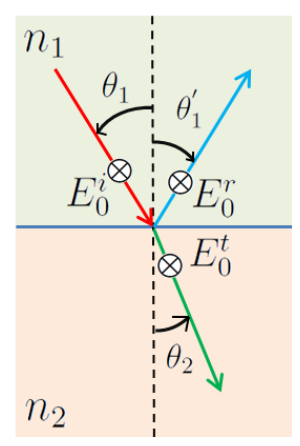

(b)

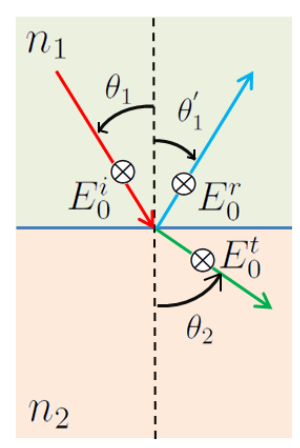

(c)

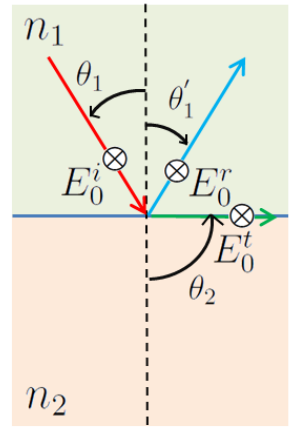

(d)

Figure 2.3.: EM wave reflection and transmission at the interface between medium 1 (intrinsic impedance $\eta_{1}$ and index of refraction $n_{1}$ ) and medium 2 (intrinsic impedance $\eta_{2}$ and index of refraction $n_{2}$ ). (a) Normal incidence. (b) Inward refraction. (c) Outward refraction. (d) critical angle (no transmission).

medium $2\left(E_{0}^{t}\right.$ and $\left.H_{0}^{t}\right)$. The mechanisms of reflection and transmission are regulated by the Fresnel laws for normal incidence, which state that:

$$
\begin{aligned}
& \rho_{\mathrm{c}}=\frac{E_{0}^{r}}{E_{0}^{i}}=\frac{\eta_{2}-\eta_{1}}{\eta_{2}+\eta_{1}}, \\
& \tau_{\mathrm{c}}=\frac{E_{0}^{t}}{E_{0}^{i}}=\frac{2 \eta_{2}}{\eta_{2}+\eta_{1}},
\end{aligned}
$$

where $\eta_{1}$ and $\eta_{2}$ are respectively the intrinsic impedances of medium 1 and 2 , and $\rho_{\mathrm{c}}$ and $\tau_{\mathrm{c}}$ identify the Fresnel reflection and transmission coefficients.

If the incident wave meets the interface between the two media with a certain angle $\theta_{1}$, as in Fig. 2.3 (b) - 2.3 (d), a portion if it is reflected back into medium 1, forming an angle $\theta_{1}^{\prime}$ with the normal to the boundary which is related to $\theta_{1}$ by the Snell's law of reflection:

$$
\theta_{1}=\theta_{1}^{\prime}
$$

while part of it will be transmitted into medium 2 changing its direction accordingly to Snell's law of refraction:

$$
\frac{\sin \theta_{2}}{\sin \theta_{1}}=\frac{v_{\mathrm{p} 2}}{v_{\mathrm{p} 1}}=\sqrt{\frac{\varepsilon_{1}^{\prime}}{\varepsilon_{2}^{\prime}},}
$$

where $v_{\mathrm{p} 1}$ and $v_{\mathrm{p} 2}$ are the phase velocities characterizing medium 1 and 2, respectively. Each medium can be characterized by a different index of refraction $n^{\mathrm{c}}$, defined as the 
ratio between the phase velocity in free space, which for a lossless medium of permittivity $\varepsilon^{\prime} \varepsilon_{0}$ can be described using the speed of light $v_{\text {lux }}$, and the phase velocity in the medium itself:

$$
n^{\mathrm{c}}=\frac{v_{\mathrm{lux}}}{v_{\mathrm{p}}}=\sqrt{\frac{\mu_{\mathrm{mag}, 0} \varepsilon^{\prime} \varepsilon_{0}}{\mu_{\mathrm{mag}, 0} \varepsilon_{0}}}=\sqrt{\varepsilon^{\prime}} .
$$

$n^{\mathrm{c}}$ quantifies the decrease of the wave's propagation velocity when it travels in a medium, with respect to free space. If the indices of refraction $n_{1}^{\mathrm{c}}$ and $n_{2}^{\mathrm{c}}$ are associated to medium 1 and 2, respectively, (2.33) becomes:

$$
\frac{\sin \theta_{2}}{\sin \theta_{1}}=\frac{v_{\mathrm{p} 2}}{v_{\mathrm{p} 1}}=\frac{n_{1}^{\mathrm{c}}}{n_{2}^{\mathrm{c}}}
$$

In particular, one can refer to inward refraction if $n_{1}^{\mathrm{c}}<n_{2}^{\mathrm{c}}$, and therefore $\theta_{1}>\theta_{2}$ (Fig. 2.3 (b)), and to outward refraction if $n_{1}^{\mathrm{c}}>n_{2}^{\mathrm{c}}$, leading to $\theta_{1}<\theta_{2}$ (Fig. 2.3 (c)). A particular case happens when $\theta_{2}=\pi / 2$, called critical angle $\theta_{c}$. In this case, the refracted wave travels on the interface between the two media, and no energy is transmitted into medium 2, as shown in Fig. 2.3 (d).

\subsection{Radar Backscattering}

In the following section the fundamental concepts of radar backscattering mechanisms, such as the scattering matrix and radar backscattering from a point or distributed target, are discussed. The radar equation is introduced, together with basic concepts of surface and volume scattering. The concept of speckle will be discussed later on in chapter 3, since its interpretation is directly related to SAR images.

\subsubsection{Scattering Matrix}

Consider an EM plane wave, polarized in both horizontal and vertical directions, illuminating a small scattering object in the far-field region. The transmitted and received electric fields $\mathbf{E}^{t}$ and $\mathbf{E}^{r}$ can be decomposed in the following way:

$$
\begin{aligned}
& \mathbf{E}^{t}=\hat{\mathbf{v}}_{\mathrm{t}} E_{\mathrm{v}}^{t}+\hat{\mathbf{h}}_{\mathrm{t}} E_{\mathrm{h}}^{t}, \\
& \mathbf{E}^{r}=\hat{\mathbf{v}}_{\mathrm{r}} E_{\mathrm{r}}^{t}+\hat{\mathbf{h}}_{\mathrm{r}} E_{\mathrm{h}}^{r},
\end{aligned}
$$

where the unit vectors $\hat{\mathbf{h}}_{\mathrm{t}}, \hat{\mathbf{h}}_{\mathrm{r}}, \hat{\mathbf{v}}_{\mathrm{t}}$, and $\hat{\mathbf{v}}_{\mathrm{r}}$ identify the directions of the horizontal and vertical components [1]. $\mathbf{E}^{r}$ and $\mathbf{E}^{t}$ are related by: 


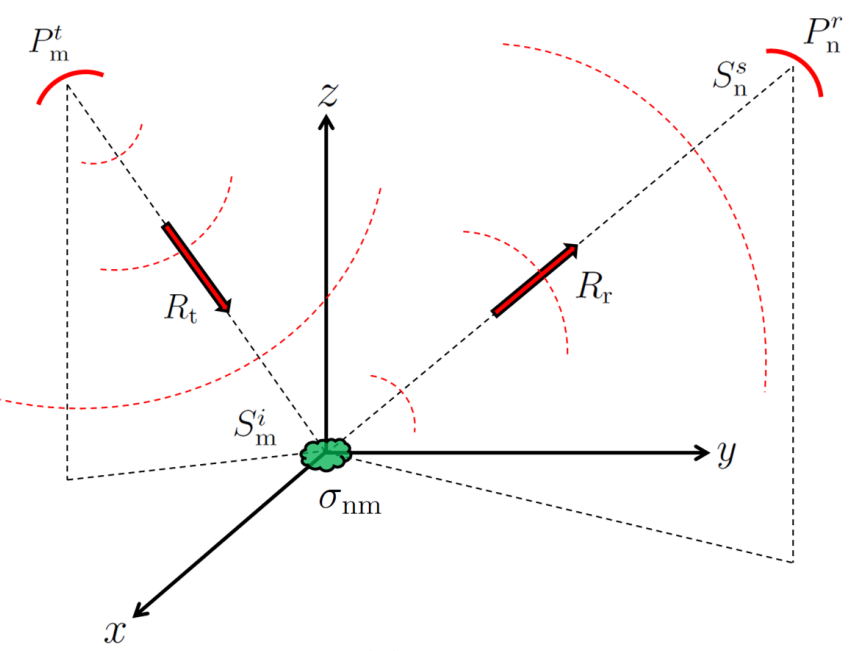

(a)

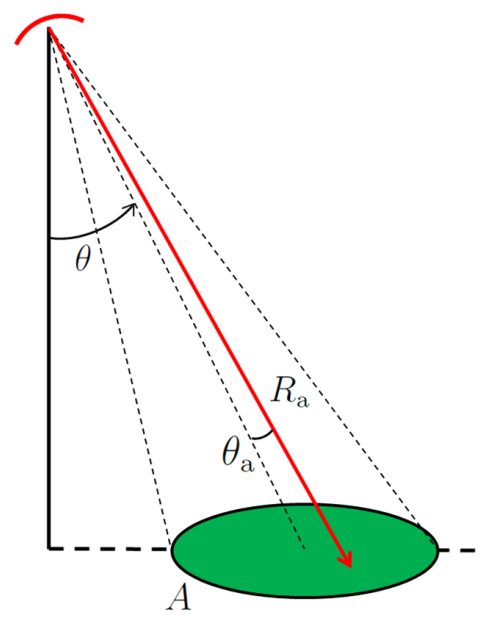

(b)

Figure 2.4.: (a) Reference geometry for the computation of the radar equation. (b) Illumination geometry for a distributed target of area $A$.

$$
\left(\begin{array}{c}
E_{\mathrm{v}}^{r} \\
E_{\mathrm{h}}^{r}
\end{array}\right)=\frac{e^{-j k R_{\mathrm{r}}}}{R_{\mathrm{r}}}\left(\begin{array}{cc}
s_{\mathrm{vv}} & s_{\mathrm{vh}} \\
s_{\mathrm{hv}} & s_{\mathrm{hh}}
\end{array}\right)\left(\begin{array}{c}
E_{\mathrm{v}}^{t} \\
E_{\mathrm{h}}^{t}
\end{array}\right)
$$

where $R_{\mathrm{r}}$ is the distance between the receiver and the illuminated object, $\left(e^{-j k R_{\mathrm{r}}} / R_{\mathrm{r}}\right)$ is the spherical propagation factor, and the matrix

$$
\mathbf{s}=\left(\begin{array}{ll}
s_{\mathrm{vv}} & s_{\mathrm{vh}} \\
s_{\mathrm{hv}} & s_{\mathrm{hh}}
\end{array}\right)
$$

is called scattering matrix. It contains the differently polarized components of the object's scattering amplitude, where, as stated in [37], for the principle of reciprocity, $s_{\mathrm{vh}}=s_{\mathrm{hv}}$.

\subsubsection{Scattering from a Point Target}

A point target is an object of small dimensions compared to the angular resolution of the radar, which means that the solid angle that it subtends is much smaller than the one subtended by the antenna beam.

Consider now the radar bistatic configuration in Fig. 2.4, composed of a transmitting m-polarized antenna, where the m-index can either correspond to linear horizontal (hindex) or vertical ( $\mathrm{v}$-index) polarization, and a receiving n-polarized one. A single point target situated at a distance equal to $R_{\mathrm{t}}$ is illuminated with an incident wave from the 
transmitting antenna, which is then scattered back along different directions. Only a portion of the reradiated $n$-polarized energy is intercepted by the receiving antenna. This process is described by the point target bistatic radar equation [38]:

$$
P_{\mathrm{n}}^{r}=\frac{P_{\mathrm{m}}^{t} G_{\mathrm{t}} G_{\mathrm{r}} \lambda^{2}}{(4 \pi)^{3} R_{\mathrm{t}}^{2} R_{\mathrm{r}}^{2}} \sigma_{\mathrm{nm}}
$$

where $P_{\mathrm{n}}^{r}$ is the received n-polarized power, $P_{\mathrm{m}}^{t}$ is the transmitted m-polarized power, $G_{\mathrm{t}}$ and $G_{\mathrm{r}}$ the transmitting and receiving antenna gains, $R_{\mathrm{r}}$ the distance between the target and the receiving antenna, and $\sigma_{\mathrm{nm}}$ the radar cross section. In case of a monostatic radar, where $G_{\mathrm{t}}=G_{\mathrm{r}}=G$ and $R_{\mathrm{t}}=R_{\mathrm{r}}=R,(2.39)$ becomes:

$$
P_{\mathrm{n}}^{r}=\frac{P_{\mathrm{m}}^{t} G^{2} \lambda^{2}}{(4 \pi)^{3} R^{4}} \sigma_{\mathrm{nm}} .
$$

The radar cross section $\sigma_{\mathrm{nm}}$ represents the strength, with which the target redirects back the illuminating energy and is defined as in [38]:

$$
\sigma_{\mathrm{nm}}=\lim _{R_{\mathrm{r}} \rightarrow \infty}\left(4 \pi R_{\mathrm{r}}^{2} \frac{S_{\mathrm{n}}^{s}}{S_{\mathrm{m}}^{i}}\right)
$$

where $S_{\mathrm{n}}^{s}$ is the scattered power density at the location of the receiving antenna and $S_{\mathrm{m}}^{i}$ is the power density illuminating the target. The limit $R_{\mathrm{r}} \rightarrow \infty$ underlines the fact that the scattered power density $S_{\mathrm{n}}^{s}$ is measured in the far-field region, where the wave front can be considered to be planar. As presented in [1], the radar cross-section in (2.41) can be expressed in terms of scattering amplitude, introduced in section 2.2.1, as:

$$
\sigma_{\mathrm{nm}}=4 \pi\left|s_{\mathrm{nm}}\right|^{2}
$$

\subsubsection{Scattering from a Distributed Target}

Distributed targets are extended targets where the contribution at the receiving antenna is given by the coherent sum of multiple reflections and where there is no predominant scattering mechanism within the resolution cell. (2.40) can therefore be extended to this case by integrating over the illuminated area $A$ :

$$
P_{\mathrm{n}}^{r}(\theta)=\iint_{A_{\sigma}} \frac{P_{\mathrm{m}}^{t} G^{2}\left(\theta_{\mathrm{a}}, \phi_{\mathrm{a}}\right) \lambda^{2}}{(4 \pi)^{3} R_{\mathrm{a}}^{4}} \sigma_{\mathrm{nm}}^{0} d A_{\sigma},
$$

where $\theta$ is the incidence angle of the boresight direction of the transmitting antenna and $\phi_{\mathrm{a}}$ is the squint angle. $\left(\theta_{\mathrm{a}}, \phi_{\mathrm{a}}\right)$ define the direction to a point inside $A$ with respect to the antenna boresight direction, and $R_{\mathrm{a}}$ is the distance between the point and the antenna. $\sigma_{\mathrm{nm}}^{0}$ represents the backscattering cross section per unit area (or simply the 


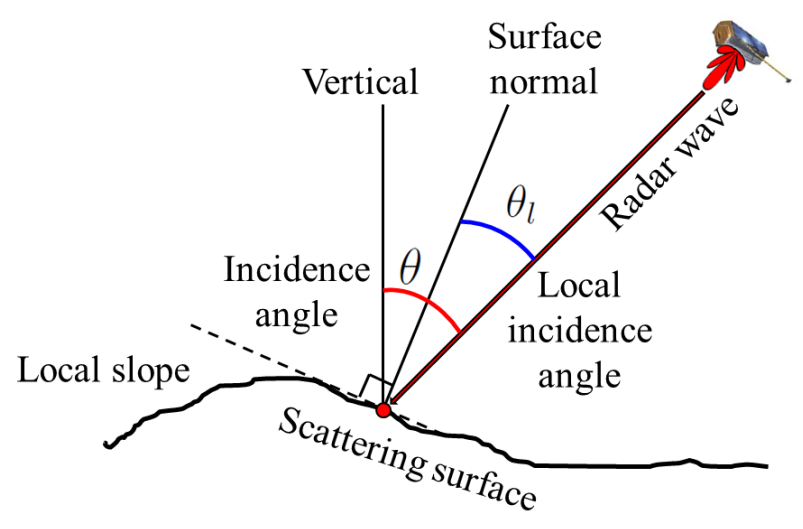

Figure 2.5.: Reference geometry for the incidence angle $\theta$ and for the local incidence angle $\theta_{l}$.

backscattering coefficient), defined as the radar cross section $\sigma_{\mathrm{nm}}$ of a distributed target of area $A_{\sigma}$, normalized with respect to $A_{\sigma}$ itself:

$$
\sigma_{\mathrm{nm}}^{0}=\sigma_{\mathrm{nm}} / A_{\sigma} .
$$

Moreover, the radar backscatter can be defined as the direct ratio between the scattered power and the incident power at ground level; in this case it is identified as $\beta_{\mathrm{nm}}$ and the backscattering coefficient can be expressed in terms of reflectivity per unit area in slant range $A_{\beta}$. It is called radar brightness $\beta_{\mathrm{nm}}^{0}$ and is related to $\sigma_{\mathrm{nm}}^{0}$ by:

$$
\beta_{\mathrm{nm}}^{0}=\frac{\beta_{\mathrm{nm}}}{A_{\beta}}=\frac{\sigma_{\mathrm{nm}}^{0}}{\sin \left(\theta_{\mathrm{l}}\right)},
$$

where $\theta_{1}$ is the local incidence angle and describes the angle between the radar wave incident direction and the normal direction to the scattering surface, as depicted in Fig. 2.5. In case of flat Earth, $\theta_{l}$ coincides with the incidence angle $\theta$.

Finally, it is also possible to express the backscattering coefficient in terms of unit area perpendicular to the antenna beam $A_{\gamma}$. In such a case, it is denoted as $\gamma_{\mathrm{nm}}^{0}$ and given by:

$$
\gamma_{\mathrm{nm}}^{0}=\beta_{\mathrm{nm}}^{0} \frac{A_{\beta}}{A_{\gamma}}=\beta_{\mathrm{nm}}^{0} \tan \theta_{\mathrm{l}} .
$$

The reference geometry for flat Earth displaying the backscatter normalization areas $A_{\sigma}, A_{\gamma}$, and $A_{\beta}$ is shown in Fig. 2.6. 


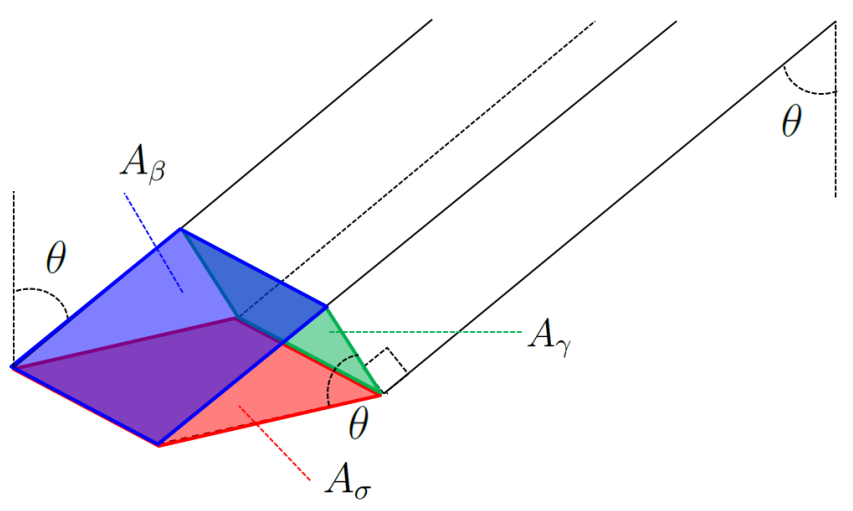

Figure 2.6.: Reference geometry for flat Earth displaying the backscatter normalization areas $A_{\sigma}, A_{\gamma}$, and $A_{\beta}$.

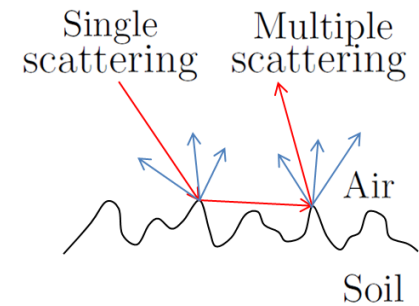

(a)

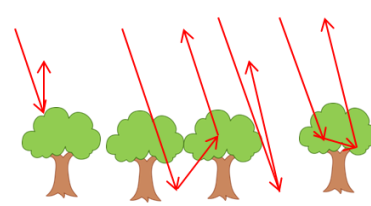

Soil

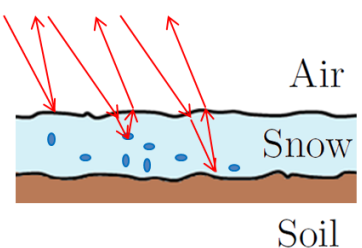

(c)

Figure 2.7.: (a) Surface scattering from a random surface, characterized by single and multiple scattering. (b) Volume scattering from vegetation canopy. (c) Surface and volume scattering from snow-covered soil.

\subsubsection{Surface and Volume Scattering}

The received backscattered radar signal is generally given by either surface or volume scattering mechanisms, or by a combination of both of them [1]:

- Surface scattering denotes the reradiated signal from the air-soil interface. It can be divided into single or multiple scattering, depending on whether the signal is directly sent back towards the radar antenna or it involves multiple reflections against other targets. An example is presented in Fig. 2.7 (a).

- Volume scattering occurs when the reradiated signal is the sum of the contributions from many individuals scatterers located at different positions within a certain volume, e.g. between the soil and the top of the canopy, as presented in Fig. 2.7 (b). Volume scattering is influenced by several factors, such as density, threedimensional orientation, shape of the targets, their dielectric composition, and the 
radar wavelength.

- Surface and volume scattering is a combination of both scattering mechanisms. Snow-covered areas are a typical example of where both surface and volume scattering mechanisms interact with each other, as shown in Fig. 2.7 (c). The snow surface directly contributes with surface scattering, while the snow layer is characterized by the presence of ice crystals which can act as reflectors, contributing to volume scattering. Depending on the conditions of the snow pack and on the wavelength, the EM wave can penetrate into the snow layer and reach the ground, where a further backscattering occurs.

\subsubsection{Chapter Remarks}

This chapter is meant to provide the reader with basic background concepts on electromagnetic plane waves and radar backscatter, needed for the understanding of the following chapters.

In particular, the reader should now be aware of the main mechanisms regulating the propagation of a TEM wave, characterized by electric and magnetic field components perpendicular to each other and to the direction of propagation.

The concepts of wave polarization and wave reflection and transmission at an interface become of fundamental importance for understanding radar backscatter and its properties.

This topic has been introduced by defining the scattering matrix and the concept of radar cross section, which quantifies the strength with which a target redirects the illuminating energy towards the receiving antenna.

Given a certain transmitted power, the antenna gain, and the acquisition geometry, the received power is regulated by the radar equation. The latter has been formulated for both a point and a distributed target. Moreover, for distributed targets, the backscattering coefficients for different projections have been derived as well.

Finally, the chapter is concluded with a brief introduction on different kinds of scattering mechanisms, divided into surface, volume, and a combination of surface and volume scattering. 


\section{Principles of SAR Imaging and Interferometry}

In this chapter the fundamental principles of Synthetic Aperture Radar and SAR interferometry are discussed.

In section 3.1, the SAR image formation is presented, starting from the acquisition geometry, until the final image focusing, absolute calibration, and geocoding. Exhaustive contributions on the subject can be found in [39], [35], [40], and [41].

In section 3.2, the attention is focused on the description across-track interferometry, which allows to retrieve a digital elevation model (DEM) starting from a pair of SAR images, acquired from two slightly different positions. A detailed compendium on the topic can be found in [2].

\subsection{SAR Image Formation}

\subsubsection{Acquisition Geometry and Resolution}

A Synthetic Aperture Radar is a side-looking radar used in remote sensing for imaging purposes. It is normally mounted on either airborne or spaceborne platforms, sending electromagnetic pulses at a defined pulse repetition frequency (PRF). The acquisition geometry is displayed in Fig. 3.1, where $x$ identifies the along-track dimension (azimuth), $y$ the across-track dimension (ground range), and $z$ the sensor hight direction. The sensor is a radar antenna with azimuth length $L_{\mathrm{a}}$ and flies along the azimuth dimension. The slant range (or simply range) dimension identifies the direction between the sensor and the target on ground. The slant range resolution $\rho_{\mathrm{rg}}$, defined as the minimum distance that allows to correctly resolve two separate targets, is proportional to the pulse duration in time $\tau_{\mathrm{rg}}$ and inversely proportional to the bandwidth $B_{\mathrm{rg}}$ of the transmitted signal:

$$
\rho_{\mathrm{rg}}=\frac{v_{\mathrm{lux}} \tau_{\mathrm{rg}}}{2}=\frac{v_{\mathrm{lux}}}{2 B_{\mathrm{rg}}},
$$

Once the slant range resolution $\rho_{\text {rg }}$ has been defined, it is possible to derive the resolution on ground as:

$$
\rho_{\mathrm{g}}=\frac{\rho_{\mathrm{rg}}}{\sin \left(\theta_{\mathrm{i}}\right)}=\frac{\rho_{\mathrm{rg}}}{\sin (\theta-\alpha)}=\frac{v_{\mathrm{lux}}}{2 B_{\mathrm{rg}} \sin (\theta-\alpha)},
$$

where $\theta_{\mathrm{i}}$ is the incidence angle with respect to the ground, $\theta$ is the one with respect to the reference plane $p_{\text {ref }}$ (e.g. the ellipsoid), and $\alpha$ is the local slope. The reference geometry 


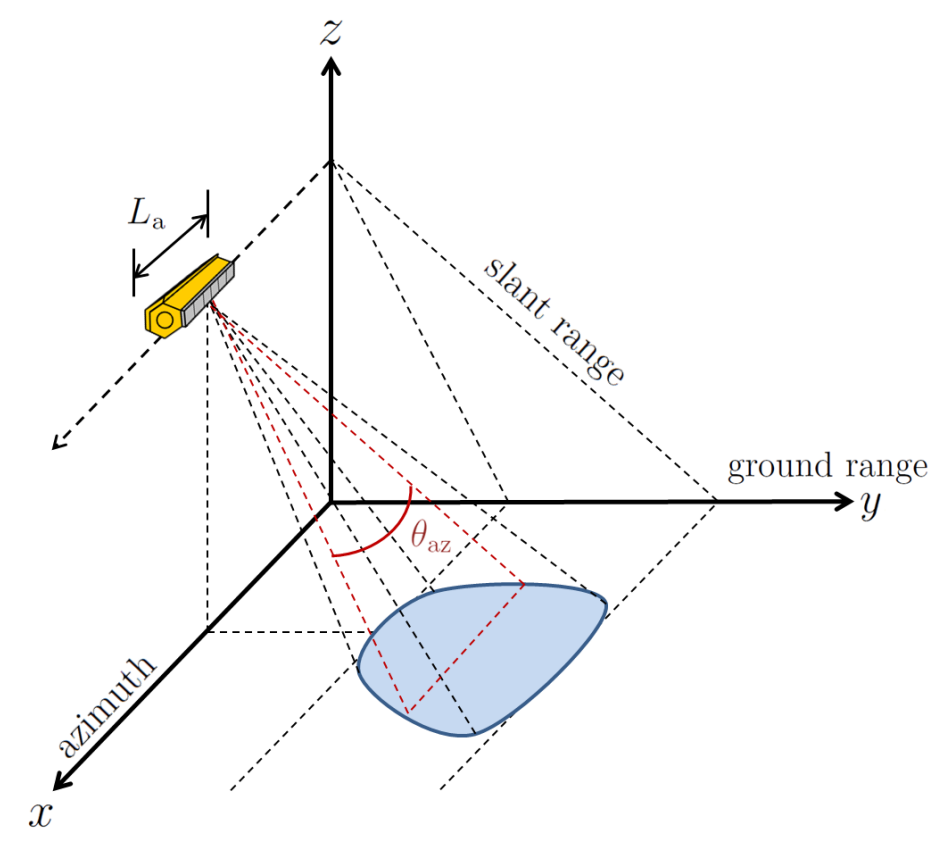

Figure 3.1.: Reference geometry for a SAR acquisition.

is presented in Fig. 3.4 (a).

The transmitted pulse $d(\tau)$ is typically a complex chirp, characterized by a constant amplitude in the time domain and given by:

$$
d(\tau)=e^{j \pi k_{\mathrm{r}} \tau^{2}} \operatorname{rect}\left(\tau / \tau_{\mathrm{rg}}\right),
$$

where $k_{\mathrm{r}}$ is the chirp rate and $\tau$ is the slant range time. An example is given in Fig. 3.2. Such an impulse is linearly frequency modulated, and it allows to spread the signal power on a wider time window, avoiding the necessity of increased transmitted power for assuring a high resolution.

The angular resolution in azimuth of a side-looking radar $\theta_{\mathrm{az}}$ (shown in Fig. 3.1) depends on the antenna beam width and is given by:

$$
\theta_{\mathrm{az}}=\frac{\lambda}{L_{\mathrm{a}}}
$$

By increasing the observation time of an object, a higher azimuth resolution $\rho_{\text {az }}$ can be achieved by exploiting the concept of the synthetic aperture $L_{\mathrm{sa}}$, which is defined as the distance that the sensor covers during a complete acquisition with duration $T_{\mathrm{o}}$. It is given by: 

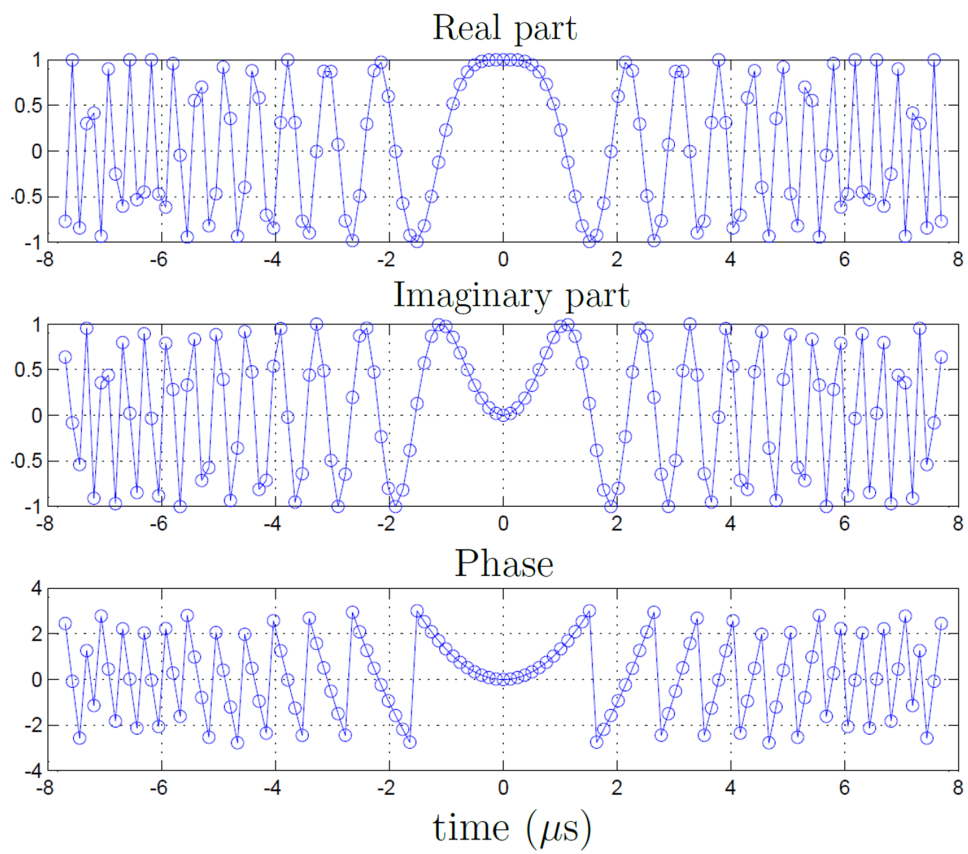

Figure 3.2.: Example of sampled complex chirp pulse.

$$
L_{\mathrm{sa}}=\frac{\lambda R_{0}}{L_{\mathrm{a}}}
$$

where $R_{0}$ represents the minimum distance between the sensor and the closest approach to the target (zero Doppler distance). In this way, the backscattered signal coming from an on-ground object is recorded during the whole observation time $T_{\mathrm{o}}$ and then reconstructed by means of a coherent sum of all the energy contributions spread along $T_{\mathrm{o}}$. This operation, called azimuth focusing, is explained in section 3.1.3.

The angular resolution $\theta_{\mathrm{sa}}$ corresponding to the synthetic aperture $L_{\mathrm{sa}}$ can be evaluated as:

$$
\theta_{\mathrm{sa}}=\frac{\lambda}{2 L_{\mathrm{sa}}}
$$

where factor 2 takes into account the two-way path of the electromagnetic wave. Hence, the enhanced azimuth resolution $\rho_{\mathrm{az}}$ is given by:

$$
\rho_{\mathrm{az}}=\theta_{\mathrm{sa}} R_{0}=\frac{L_{\mathrm{a}}}{2} .
$$

By assuming now a rectilinear geometry, where the sensor's orbit is considered to be linear, and the start-stop approximation, where both sensor and scatterers remain steady during both transmission and reception, the signature left by a single point target within 


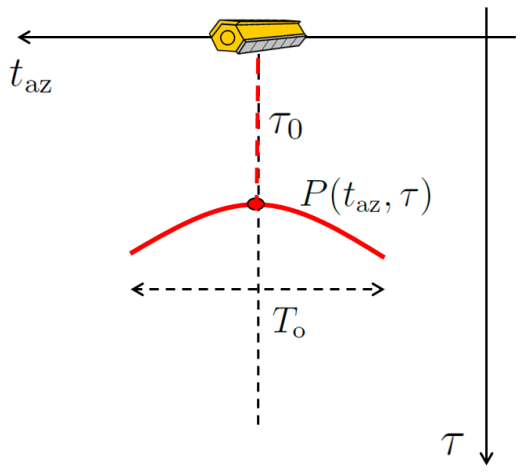

(a)

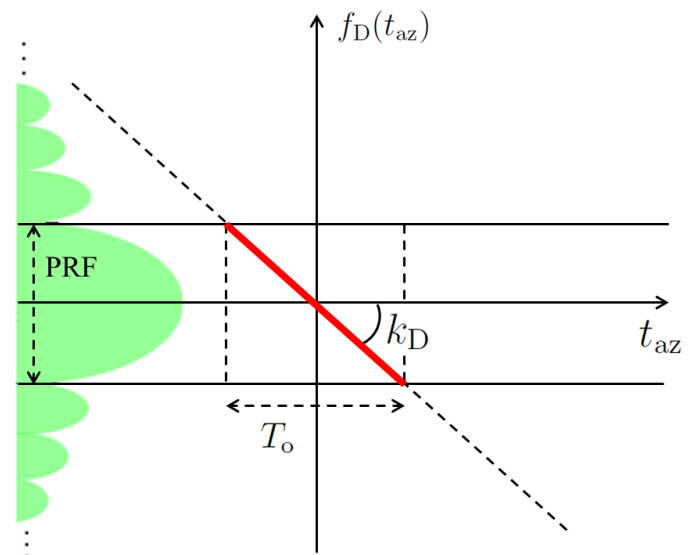

(b)

Figure 3.3.: (a) The hodograph: the signature of a point target left within the acquired SAR data and subjected to range migration. (b) Doppler history of a point target in the azimuth time/Doppler frequency plane.

the acquired data matrix $P\left(t_{\mathrm{az}}, \tau\right)$ (called hodograph) is simplified in Fig. 3.3 (a). For each sensor position along the azimuth time dimension $t_{\mathrm{az}}$, the range time $\tau$ between the sensor and $P\left(t_{\mathrm{az}}, \tau\right)$ is given by:

$$
\tau=\frac{2}{v_{\text {lux }}} R\left(t_{\mathrm{az}}\right) .
$$

$R\left(t_{\mathrm{az}}\right)$ is defined as:

$$
R\left(t_{\mathrm{az}}\right)=\sqrt{R_{0}^{2}+\left(v_{\mathrm{s}} t_{\mathrm{az}}\right)^{2}},
$$

where $v_{\mathrm{s}}$ is the sensor speed and $R_{0}=\left(v_{\text {lux }} / 2\right) \tau_{0}$, being $\tau_{0}$ the range time associated to the zero Doppler position of the target. (3.9) identifies an hyperbole, whose summit corresponds to the target position in SAR geometry. The shift of a target's signature in slant range depending on the sensor's azimuth position is also known as range migration. The backscattered energy from a target, spread over the hodograph, is used to reconstruct the target by applying the azimuth focusing, as explained in section 3.1.4.

By using a Taylor approximation, (3.9) can be simplified with a parabolic function as:

$$
R\left(t_{\mathrm{az}}\right) \simeq R_{0}+\frac{v_{\mathrm{s}}^{2}}{2 R_{0}} t_{\mathrm{az}}^{2} .
$$

The phase in azimuth of the impulse response from the considered target can now be obtained from the hodograph as: 


$$
\phi\left(t_{\mathrm{az}}\right)=-\frac{4 \pi}{\lambda} R\left(t_{\mathrm{az}}\right)=-\frac{4 \pi}{\lambda}\left(R_{0}+\frac{\left(v_{\mathrm{s}} t_{\mathrm{az}}\right)^{2}}{2 R_{0}}\right) .
$$

The instantaneous frequency $f_{\mathrm{D}}\left(t_{\mathrm{az}}\right)$, called Doppler frequency, is obtained by differentiating (3.11) with respect to the azimuth time:

$$
f_{\mathrm{D}}\left(t_{\mathrm{az}}\right)=\frac{1}{2 \pi} \frac{\partial \phi\left(t_{\mathrm{az}}\right)}{\partial t_{\mathrm{az}}}=-\frac{2 v_{\mathrm{s}}^{2}}{\lambda R_{0}} t_{\mathrm{az}}
$$

Similarly, the derivative of the Doppler frequency $k_{\mathrm{D}}$ is called Doppler rate and is given by:

$$
k_{\mathrm{D}}=\frac{\partial f_{\mathrm{D}}\left(t_{\mathrm{az}}\right)}{\partial t_{\mathrm{az}}}=-\frac{2 v_{\mathrm{s}}^{2}}{\lambda R_{0}} .
$$

Finally, the linear relationship between the azimuth time $t_{\mathrm{az}}$ and the frequency $f_{\mathrm{D}}\left(t_{\mathrm{az}}\right)$ is called Doppler history of a target and is displayed in Fig. 3.3 (b). The observable Doppler history of a target is limited by the observation time along the azimuth time dimension and by the azimuth antenna pattern beam width (depicted in green) in the Doppler frequency domain.

\subsubsection{Perspective Deformations}

The regular sampling of distances in slant range causes a variable sampling on ground, which follows (3.2). The effect is a series of geometric distortions, which can be explained by considering the geometry in Fig. 3.4 (a). Slopes oriented towards the satellite are concentrated inside a few pixels and they appear closer to the sensor (foreshortening). In the presence of slopes where $\theta<\alpha<\pi$, the top of a mountain appears closer to the sensor than the bottom and their positions are switched inside the SAR image. This phenomenon is known as layover. On the other hand, negative slopes where $-\pi<\alpha<-\pi+\theta$ (descents) are not illuminated by the sensor, appearing as black shadows within the image. Shadow and layover phenomena are described in Fig. 3.4 (b).

\subsubsection{Range Focusing}

The signal focusing in the slant range dimension is performed in base band by applying a matched filter to the received signal in range. Such an operation maximizes the signal-to-noise ratio (SNR) by correlating the received pulse $s_{\mathrm{r}}(\tau)$ with a replica of the transmitted chirp $d(\tau)$ as: 


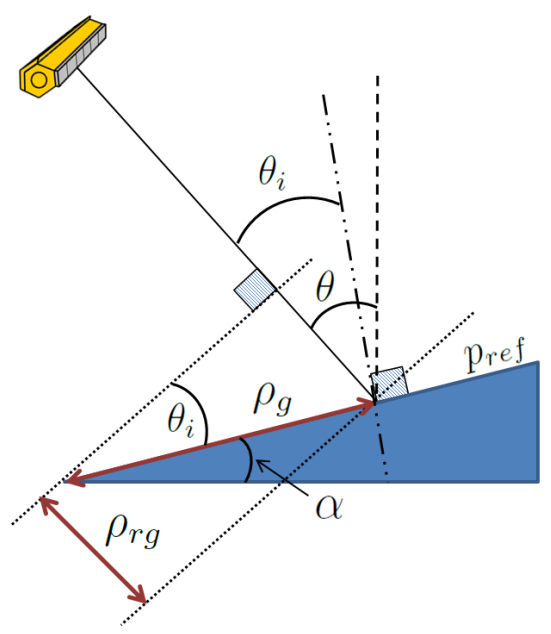

(a)

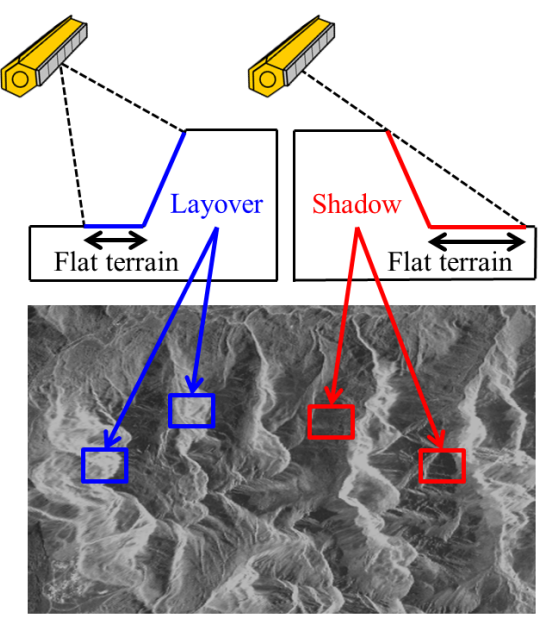

(b)

Figure 3.4.: (a) Reference geometry for the computation of the ground range resolution and for the definition of geometric distortions. (b) Description of shadow and layover effects within a SAR image.

$$
s_{\mathrm{cr}}(\tau)=\int_{-\infty}^{\infty} s_{\mathrm{r}}(\tau) d(t-\tau) d t=\tau_{\mathrm{rg}} \operatorname{sinc}\left(k_{\mathrm{r}} \tau_{\mathrm{rg}} \tau\right)
$$

where $s_{\mathrm{cr}}(\tau)$ represents the range compressed signal, which is approximately given by a sinc function, whose geometric resolution at half-power points $(-3 \mathrm{~dB})$ equals $(3.1)$, as demonstrated in [42].

\subsubsection{Azimuth Focusing}

As inferred from Fig. 3.3 (a), the energy of a single point target is spread along the azimuth dimension, following the law of range migration. Therefore, it is necessary to concentrate it in the correct position (zero Doppler position), in order to correctly reconstruct the target. Accordingly to (3.11), the received signal in azimuth can be written as:

$$
s_{\mathrm{az}}\left(t_{\mathrm{az}}\right)=e^{-j \phi\left(t_{\mathrm{az}}\right)}=e^{\left(j \frac{4 \pi}{\lambda} R_{0}\right)} e^{\left(j \frac{4 \pi}{\lambda} \frac{\left(v_{\mathrm{s}} t_{\mathrm{az}}\right)^{2}}{2 R_{0}}\right)} .
$$

The first exponential can be seen as a complex constant, while the second one is a chirp generated by the acquisition geometry of a SAR image itself. As presented in [43], the complex constant can be neglected and the azimuth chirp sampled with frequency PRF as: 


$$
t_{\mathrm{az}}=n d_{\mathrm{az}}=1 / \mathrm{PRF},
$$

where $d_{\mathrm{az}}$ is the azimuth pulse repetition time and $n \in\left[-N_{0} / 2, N_{0} / 2\right]$, being $N_{0}$ the azimuth chirp length in samples. Once the complex constant has been omitted, (3.15) can be rewritten as:

$$
s_{\mathrm{az}}\left(n d_{\mathrm{az}}\right)=e^{\left(j \frac{2 \pi}{\lambda R_{0}}\left(v_{\mathrm{s}} n d_{\mathrm{az}}\right)^{2}\right)} .
$$

By applying a coherent correlation, as presented in [43], the azimuth compressed pulse $s_{\mathrm{ac}}\left(n d_{\mathrm{az}}\right)$ is given by the periodic Dirichlet function:

$$
s_{\mathrm{ac}}\left(n d_{\mathrm{az}}\right)=\frac{\sin \left(\frac{2 \pi\left(v_{\mathrm{s}} d_{\mathrm{az}}\right)^{2}}{\lambda R_{0}} n(N+1-n)\right)}{\sin \left(\frac{2 \pi\left(v_{\mathrm{s}} d_{\mathrm{az}}\right)^{2}}{\lambda R_{0}} n\right)} .
$$

By substituting $t_{\mathrm{az}}$ in (3.18) again, as in (3.16), the following expression is obtained:

$$
s_{\mathrm{ac}}\left(t_{\mathrm{az}}\right)=\frac{\sin \left(\frac{2 \pi v_{\mathrm{s}}^{2} d_{\mathrm{az}}}{\lambda R_{0}} t_{\mathrm{az}}\left(N+1-\frac{t_{\mathrm{az}}}{d_{\mathrm{az}}}\right)\right)}{\sin \left(\frac{2 \pi v_{\mathrm{s}}^{2} d_{\mathrm{az}} t_{\mathrm{az}}}{\lambda R_{0}}\right)} .
$$

The characteristic periodicity of $s_{\mathrm{ac}}\left(t_{\mathrm{az}}\right)$ define the position of azimuth ambiguities within the focused image. The choice of the PRF to be used represents therefore a key parameter for the minimization of the azimuth ambiguities within the final SAR image.

\subsubsection{Image Speckle and Multilooking}

The concept of image speckle describes the pixel-to-pixel intensity fluctuation detectable in a SAR image, even though the terrain presents the same characteristics. An example is visible in Fig. 3.5 (a), depicting an agricultural area acquired by the TerraSAR-X sensor. This phenomenon is particulary visible over vegetated areas (forests and agricultural fields) and rough surfaces. It is due to the coherent sum of scattering contributions within the resolution cell, where all scatterers are assumed to be statistically independent and with comparable scattering strengths. Moreover, speckle can be statistically described as a realization of a stationary process, with circular complex Gaussian distribution. By assuming such a model, the intensity of the backscattered signal $\sigma^{0}$ can be modeled as Rayleigh-distributed, whose probability density function (pdf) is given by:

$$
P\left(\sigma^{0}\right)=\frac{1}{\mathrm{E}\left[\sigma^{0}\right]} e^{-\sigma^{0} / \mathrm{E}\left[\sigma^{0}\right]},
$$




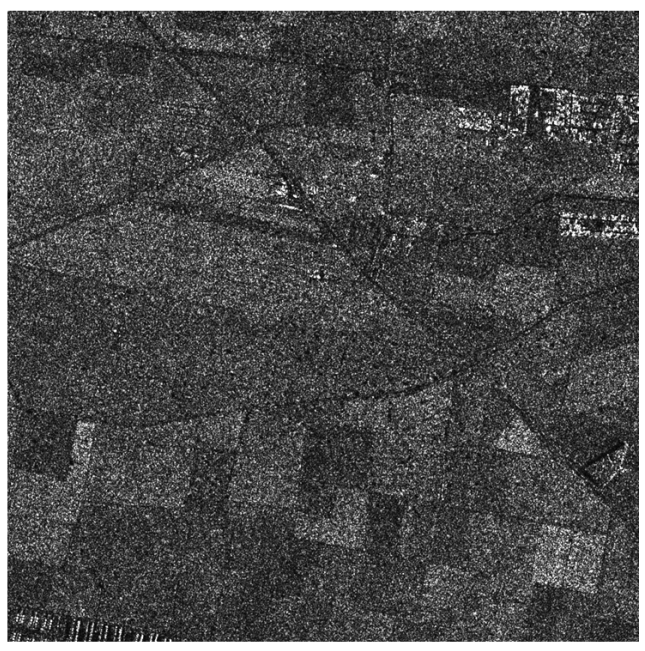

(a)

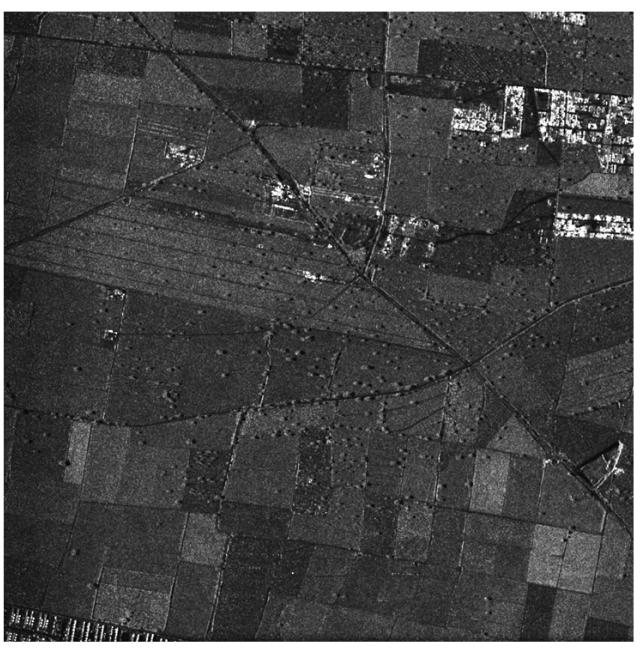

(b)

Figure 3.5.: (a) SAR image an agricultural area acquired by TerraSAR-X. (b) Speckle removal by temporal multilooking of 32 TerraSAR-X images acquired over the same ground area.

where $\mathrm{E}\left[\sigma^{0}\right]$ is the reflectivity mean value.

Recalling now the definition of the coefficient of variation of a random variable $\mathrm{x}$ as:

$$
V=\sigma_{\mathrm{x}} / \mathrm{E}[x],
$$

where $\sigma_{\mathrm{x}}$ is the standard deviation and $\mathrm{E}[x]$ the mean value, it is possible to define the radiometric resolution as:

$$
\rho_{\text {rad }}=10 \log (1+V),
$$

which quantifies the fluctuation of the backscattered signal and therefore the accuracy of its measurement. Since the variance related to $\sigma^{0}$ is given by $\left(\mathrm{E}\left[\sigma^{0}\right]\right)^{2}$, if (3.20) is valid, then $V=1$ and $\rho_{\text {rad }}=3 \mathrm{~dB}$. In order to reduce the radiometric resolution and improve the determination of $\sigma^{0}$, it is possible to consider the incoherent sum of $N_{\mathrm{L}}$ measurements for which (3.20) is valid, obtaining:

$$
V_{\mathrm{N}}=\frac{1}{\sqrt{N_{\mathrm{L}}}}
$$

and therefore:

$$
\rho_{\mathrm{rad}}=10 \log \left(1+\frac{1}{\sqrt{N_{\mathrm{L}}}}\right)
$$


Such process is called multilooking and $N_{\mathrm{L}}$ is the number of looks. If performed in the spatial dimension, it improves the radiometric resolution at the expense of a lower spatial one. Alternatively, it can be performed in the temporal dimension, if a stack of images over the same ground area is available. In this way, the radiometric resolution is improved maintaining the initial spatial resolution.

The choice between spatial and temporal multilooking may also be driven by the type of considered application. For example, temporal multi-looking could cause the loss of information when monitoring temporal changes. On the other hand, spatial multilooking could not be appropriate for edge detection applications, given the blurred effect introduced by spatial averaging.

An example of speckle removal by temporal multilooking is presented in Fig. 3.5 (b) where 32 SAR images acquired by TerraSAR-X in a time span of almost two years have been incoherently averaged as if they corresponded to different looks. The result is a despeckled image, with respect to the single one displayed in Fig. 3.5 (a), where detailed features can be clearly recognized.

\subsubsection{SAR Acquisition Modes}

The classical acquisition mode of SAR, addressed in the current chapter in order to explain the image formation principles, is commonly known as StripMap mode (Fig. 3.6 (a)). It involves the continuous acquisition of radar pulses within a certain incidence angle range, without changing the orientation of the antenna beam during an acquisition. The dimension of the illuminated swath on ground in the range dimension typically varies between $30 \mathrm{~km}$ and $100 \mathrm{~km}$.

In the last years, several advanced and more complex acquisition modes have been developed, in order to satisfy different necessities. The Spotlight mode, illustrated in Fig. 3.6 (b), allows to increase the synthetic aperture by electronically steering the antenna beam in the azimuth direction [44]. However, a continuous illumination of the ground, as in StripMap mode, is not possible. A higher resolution is achieved at the expense of a reduced coverage (typical scene size of about $15 \mathrm{~km}$ ).

In order to obtain wide swaths, between $100 \mathrm{~km}$ and $500 \mathrm{~km}$, burst modes have been implemented, such as ScanSAR [45] and TopSAR [46], presented in Fig. 3.6 (c) and Fig. 3.6 (d), respectively. The ScanSAR mode consists in the successive illumination of several parallel swaths. A high coverage is obtained at the expense of a reduced resolution and a mosaicking operation is required to join the different subswaths in range and azimuth dimensions. On the other hand, the TopSAR mode adds an azimuth beam steering of the antenna pattern, in order to improve the resolution and the image quality. Both ScanSAR and TopSAR modes are considered to be quite-continuous acquisition modes, since continuous strips on ground are composed together during the data processing phase. All modes can operate in different polarizations, and have interferometric capabilities (see 


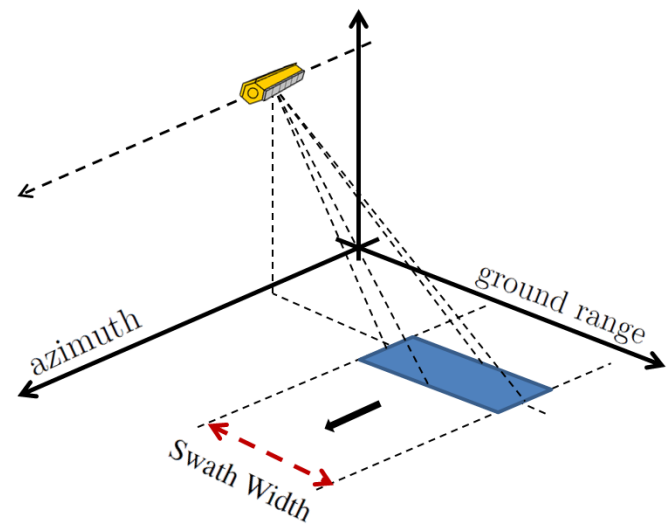

(a)

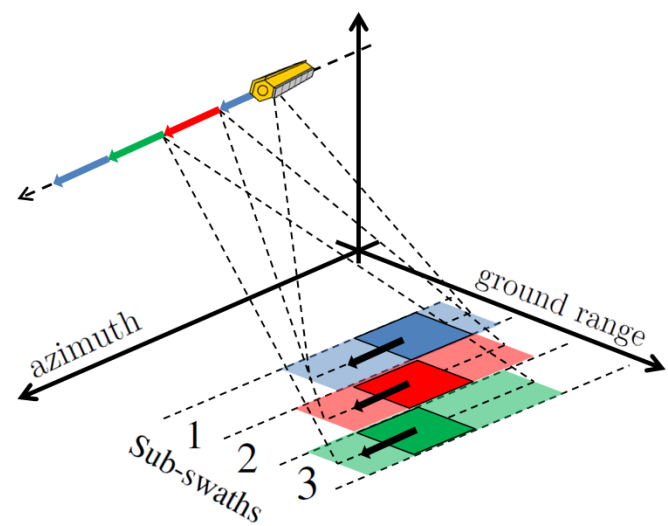

(c)

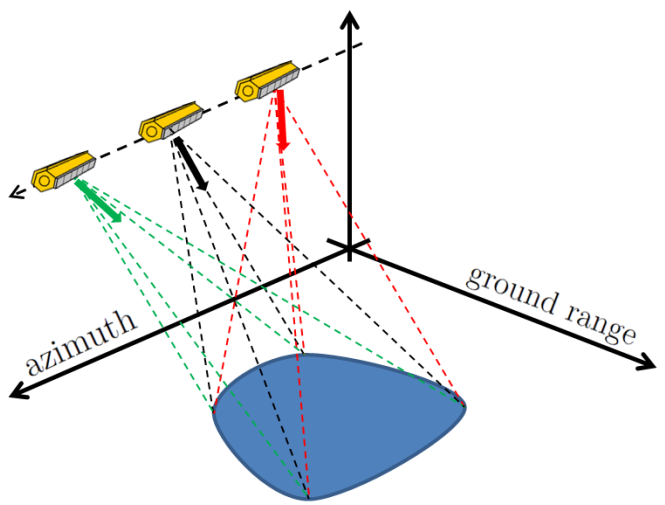

(b)

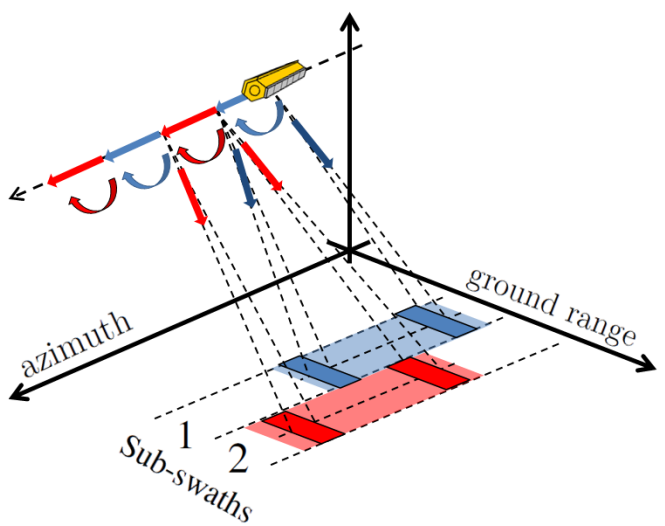

(d)

Figure 3.6.: Simplified geometries for different SAR acquisition modes. (a) StripMap mode, (b) Spotlight mode, (c) ScanSAR mode, (d) TopSAR mode.

section 3.2).

\subsubsection{Absolute Calibration of SAR Images}

The absolute calibration of a SAR sensor allows to translate the focused signal amplitude (called digital number $D N_{\mathrm{nm}}$, where indices $\mathrm{n}$ and $\mathrm{m}$ identify the transmitting and receiving polarizations, respectively) to a physical quantity, such as the scattering coefficient. It is typically performed using reference targets on ground, such as corner reflectors (passive calibrators, Fig. 3.7 (a)) and transponders (active calibrators), whose reference radar cross-section $\sigma_{\mathrm{nm}}^{r e f}$ is known a priori [1]. The typical bi-dimensional signature of a corner reflector within a focused SAR image is presented in Fig. 3.7 (b). By 


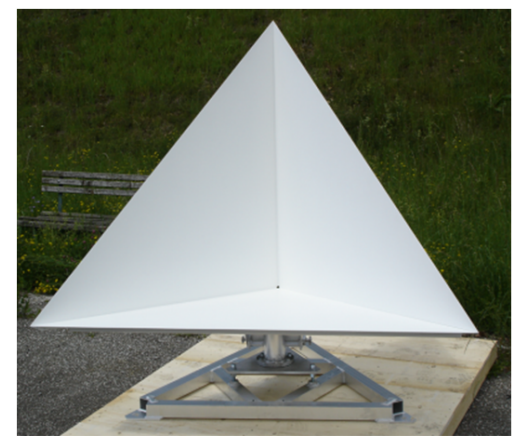

(a)

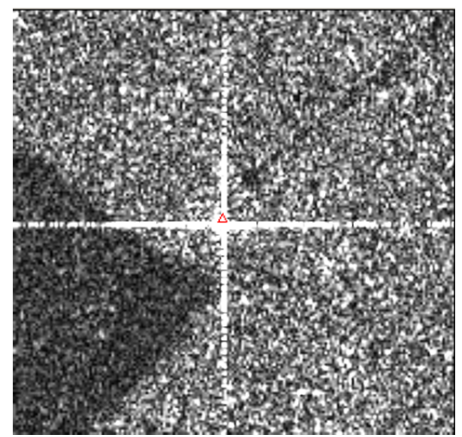

(b)

Figure 3.7.: (a) Trihedral corner reflector used as reference target for absolute calibration. (b) Bi-dimensional target response of a corner reflector within a focused SAR image, surrounded by speckle.

comparing $\sigma_{\mathrm{nm}}^{r e f}$ to the one evaluated from the corresponding focused target within the SAR image, it is possible to derive an absolute calibration constant $K_{\mathrm{nm}}^{a b s}$, which is used to estimate the radar brightness $\beta_{\mathrm{nm}}^{0}$ as:

$$
\beta_{\mathrm{nm}}^{0}=K_{\mathrm{nm}}^{a b s} D N_{\mathrm{nm}}^{2} .
$$

\subsubsection{SAR Image Geocoding}

Geocoding determines the correspondence between a pixel in the SAR image range/azimuth reference system and its position on ground in a cartesian reference system $(x, y, z)$ [47].

The most frequently used cartesian reference system is characterized by $x$ and $y$ lying on the Equatorial plane, and $z$ oriented towards the north pole. The reference geometry is presented in Fig. 3.8. The $\mathbf{P}$ vector identifies the target's position in SAR coodinates while $\mathbf{S}$ represents the satellite position, usually given in terms of its position along the orbit, called state vector. Since a SAR acquisition is bi-dimensional, additional external information is needed, in order to localize target $\mathbf{P}$ in a three-dimensional space. Such information is typically derived from a digital elevation model (DEM) of the ground, which defines the altitude of a point lying on the surface of the Earth, typically modeled with an ellipsoid.

The geolocation of $\mathbf{P}$ is obtained by solving a three-equations system:

1. The range equation defines the distance between the sensor and the target:

$$
|\mathbf{S}-\mathbf{P}|=\frac{v_{\mathrm{lux}} \tau_{\mathrm{p}}}{2},
$$




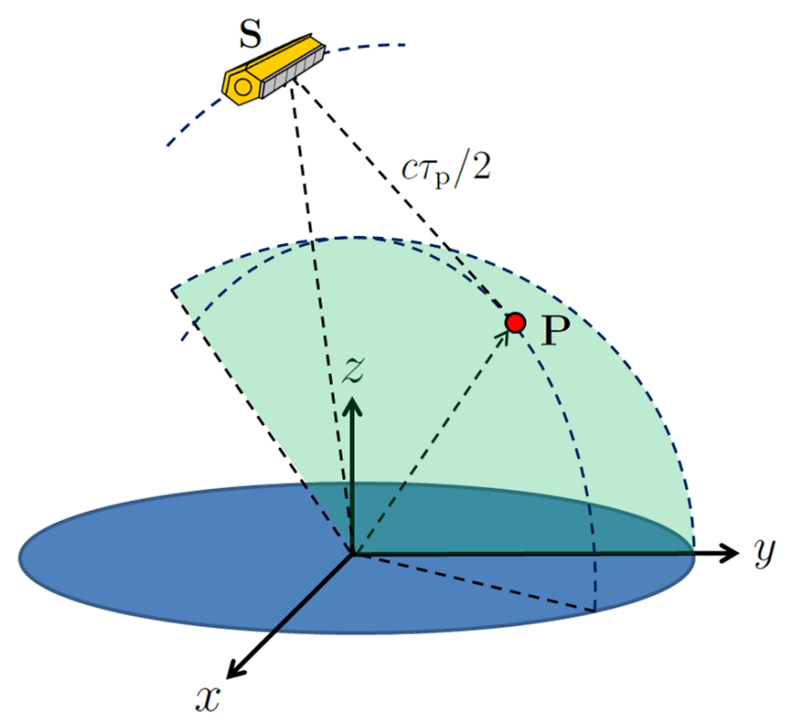

Figure 3.8.: Reference geometry in $(x, y, z)$ coordinates for SAR image geocoding.

and localizes $\mathbf{P}$ on a sphere of radius $v_{\text {lux }} \tau_{\mathrm{p}} / 2$, where $\tau_{\mathrm{p}}$ is the range time of $\mathbf{P}$.

2. The Doppler equation describes the shift due to the Doppler effect between the sensor and the target:

$$
\dot{\mathbf{S}} \frac{\mathbf{S}-\mathbf{P}}{|\mathbf{S}-\mathbf{P}|}=-\frac{\lambda}{2} f_{\mathrm{D}}
$$

where $f_{\mathrm{D}}$ is the Doppler frequency. In case of zero Doppler focusing, (3.27) becomes $\dot{\mathbf{S}}(\mathbf{S}-\mathbf{P})=0$.

3. The equation which identifies a point on the ellipsoid:

$$
\frac{p_{\mathrm{x}}^{2}+p_{\mathrm{y}}^{2}}{\left(R_{\mathrm{e}}+h_{\mathrm{p}}\right)^{2}}+\frac{p_{\mathrm{z}}^{2}}{R_{\mathrm{p}}^{2}}=1,
$$

where $\left(p_{\mathrm{x}}, p_{\mathrm{y}}, p_{\mathrm{z}}\right)$ are the Cartesian components of $\mathbf{P}, R_{\mathrm{e}}$ and $R_{\mathrm{p}}$ are the equatorial and polar Earth radius, respectively, and $h_{\mathrm{p}}$ is the geodetic height of $\mathbf{P}$, which can be obtained from a DEM. 


\subsection{SAR Interferometry}

The main objective of SAR interferometry is to derive the ground topography information by properly combining two images of the same area acquired from different positions. A first overview of the fundamental concepts and applications can be found in [48] and [49].

The fundamental principle of SAR interferometry resides in the fact that a difference in the height of two targets on ground, observed from two distinct positions in the plane perpendicular to the flight direction (across-track interferometry), corresponds to a change in the phase difference of the received signal coming from such an object.

Two different kind of acquisitions can be carried out for estimating the ground topography:

- Single-pass interferometry, where two images over the same area are recorded within a single survey. In this case a high and constant quality of the final DEM can be expected, since temporal decorrelation does not affect the final performance, as explained in section 3.2.4. The major disadvantage of such an approach is the complexity and cost of the deployment of a multi-receiver.

- Repeat-pass interferometry, where the interferometric images pair is acquired during two different passes. In this case, conventional single-receiver SAR sensors can be used. Such a configuration is subjected to atmospheric changes, which cause delay variations, and temporal decorrelation.

In the case of along-track interferometry, a compensation for the across-track baseline is performed, as if the acquisitions were carried out with a null distance between the two sensors in the plane perpendicular to the flight direction. In this case, the interferometric phase can be exploited to estimate changes in the illuminated scene on ground between the two passes, as presented in [50], [51].

In this section a short review of across-track SAR interferometry is presented, concentrating on the generation of digital elevation models (DEM).

\subsubsection{From the Acquisition Geometry to the Height Retrieval}

Consider two SAR images, acquired over the same area by two sensors $S 1$ and $S 2$ in a dual-pass interferometric configuration, as presented in Fig. 3.9. The distance between the two sensors in the plane perpendicular to the orbit is called baseline $B$. Its projections, perpendicular and parallel to the slant range dimension, are the normal baseline $B_{\perp}$ and the parallel baseline $B_{\|}$, respectively. They are oriented at an angle $\beta$ with respect to the local horizontal.

Interferometry relies on the use of the phase information and the parallel-ray approximation must be assumed, which states that: 


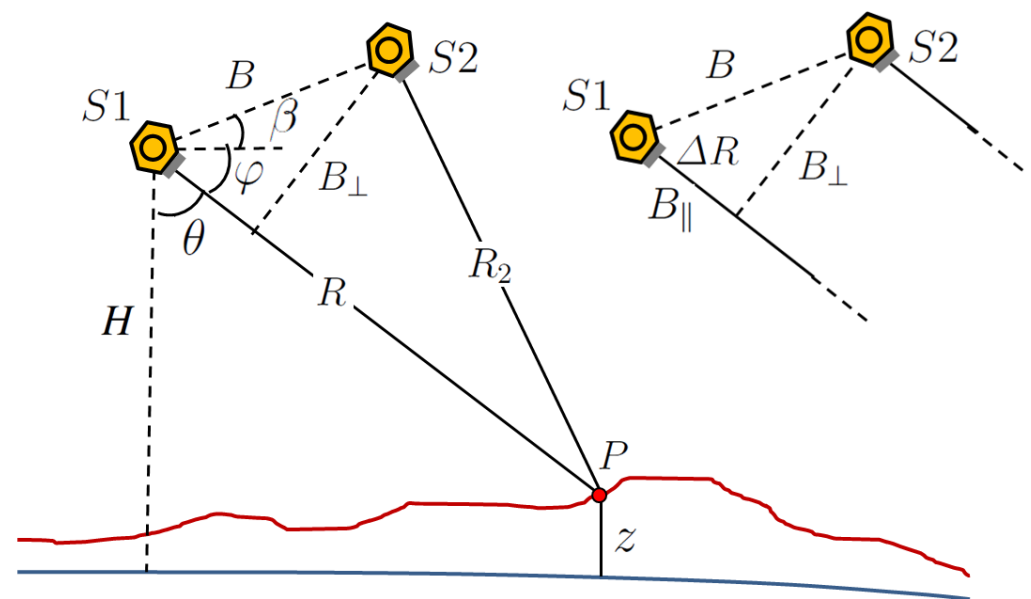

Figure 3.9.: Reference geometry for across-track interferometry. The blue curve identifies the Ellipsoid.

$$
\left|R-R_{2}\right| \ll R .
$$

Considering a monostatic configuration, where both radar sensors independently acquire an image of the same ground area, the distances between $S_{1}$ and $S_{2}$ and a point target $P$ are equal to $R$ and $R_{2}$, while the two-way path length is equal to $2 R$ and $2 R-2 \Delta R$, respectively. In a bistatic configuration, where only one radar sensor transmits $\left(S_{1}\right)$ and both of them receive, the two-way path between $P$ and $S_{2}$ is equal to $2 R-\Delta R$.

The $\varphi$ angle is given by:

$$
\varphi=\frac{\pi}{2}-\theta
$$

and the law of cosines gives:

$$
(R-\Delta R)^{2}=R^{2}+B^{2}-2 B R \cos (\varphi+\beta) .
$$

since $\Delta R \ll R$ the parallel-ray approximation can be applied, leading to:

$$
\Delta R=B \sin (\theta-\beta) .
$$

Moreover, the topographic height $z$, referred to the Ellipsoid, can be derived as:

$$
z=H-R \sin (\varphi),
$$


where $H$ is the height of $S 1$.

For a monostatic configuration, the phase of a complex pixel within the SAR image $u_{1}$, acquired by $S 1$, is given by:

$$
\phi_{1}=-\frac{4 \pi}{\lambda} R+\phi_{o b j, 1},
$$

and for image $u_{2}$, acquired by $S 2$ :

$$
\phi_{2}=-\frac{4 \pi}{\lambda}(R-\Delta R)+\phi_{o b j, 2},
$$

where $\phi_{o b j, 1}$ and $\phi_{o b j, 2}$ are the target's phase in image $u_{1}$ and $u_{2}$, respectively. By assuming $\phi_{o b j, 1}=\phi_{o b j, 2}$, the interferometric phase $\phi_{\mathrm{int}}$ is given by:

$$
\phi_{\text {int }}=\phi_{1}-\phi_{2}=-\frac{4 \pi}{\lambda} \Delta R \text {. }
$$

For a bistatic configuration (3.36) becomes:

$$
\phi_{\text {int }}=-\frac{2 \pi}{\lambda} \Delta R
$$

The equation above allows for the determination of $\Delta R$ from the interferometric phase $\phi_{\text {int }}$.

The location of the considered target in the zero Doppler plane can now be retrieved by finding the interception between the circles identified by:

$$
\begin{aligned}
R & =\text { constant } \\
\Delta R & =\text { constant }
\end{aligned}
$$

\subsubsection{The Interferogram}

The two-dimensional image of the interferometric phase $\phi_{\text {int }}$ is called interferogram. Before its computation, following (3.36), it is necessary to properly coregister the interferometric SAR images pair $u_{1}$ and $u_{2}$. The image taken as reference is commonly called master while the other one is called slave and is coregistered to the master.

Typically, coregistration is performed by cross-correlating small portions of the master and slave images, where relative shifts between the two can then be estimated from the location of the cross-correlation's maximum.

Spatial multilooking can be applied as well, in order to improve the estimated phase accuracy, at the cost of a lower spatial resolution, as explained in section 3.1.5.

Once the interferogram has been generated, the interferometric phase $\phi_{\text {int }}$ can be decomposed into two components:

$$
\phi_{\mathrm{int}}=\phi_{\mathrm{topo}}+\phi_{\mathrm{fe}}
$$




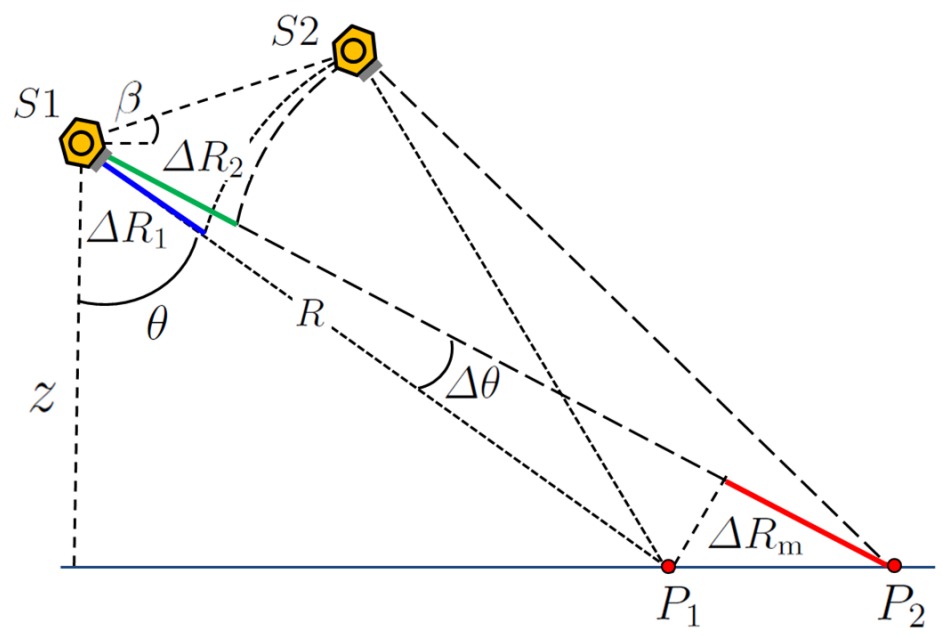

Figure 3.10.: Reference geometry for the evaluation of the flat Earth phase component.

where $\phi_{\text {topo }}$ is the topographic phase difference and $\phi_{\mathrm{fe}}$ is the flat Earth component. The latter accounts for the interferometric phase difference occurring between two scatterers at the same topographic height, but at different positions with respect to the radar sensor. The reference geometry, describing $\phi_{\mathrm{fe}}$, is presented in Fig. 3.10.

Consider now two targets on ground $P_{1}$ and $P_{2}$, characterized by the same topographic height. In this case, the interferometric phase for $P_{1}$ is:

$$
\phi_{\text {int }}^{1}=-\frac{4 \pi}{\lambda} \Delta R_{1}=-\frac{4 \pi}{\lambda} B_{\perp} \sin (\theta-\beta),
$$

and for $P_{2}$ is:

$$
\phi_{\text {int }}^{2}=-\frac{4 \pi}{\lambda} \Delta R_{2}=-\frac{4 \pi}{\lambda} B_{\perp} \sin (\theta+\Delta \theta-\beta) \text { for } P_{2} .
$$

By evaluating the phase difference $\Delta \phi$ between $P_{2}$ and $P_{1}$ as:

$$
\phi_{\text {int }}^{2}-\phi_{\text {int }}^{1}=-\frac{4 \pi}{\lambda}[B \sin (\theta+\Delta \theta-\beta)-B \sin (\theta-\beta)],
$$

a residual component remains, even though both targets have the same topographic height. Such component is exactly $\phi_{\mathrm{fe}}$ and it depends on the difference between $\Delta R_{1}$ and $\Delta R_{2}$. Starting from (3.42), $\phi_{\mathrm{fe}}$ can be expressed as:

$$
\phi_{\mathrm{fe}}=\Delta \phi=\frac{4 \pi}{\lambda} B \cos (\theta-\alpha) \Delta \theta \simeq \frac{4 \pi}{\lambda} \frac{B \cos (\theta-\alpha) \Delta R_{\mathrm{m}}}{R \tan (\theta)},
$$




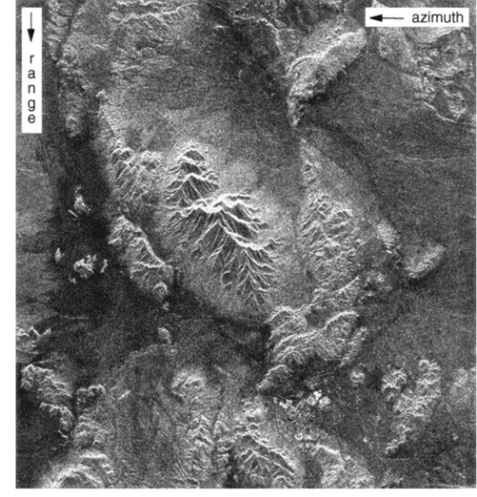

(a)

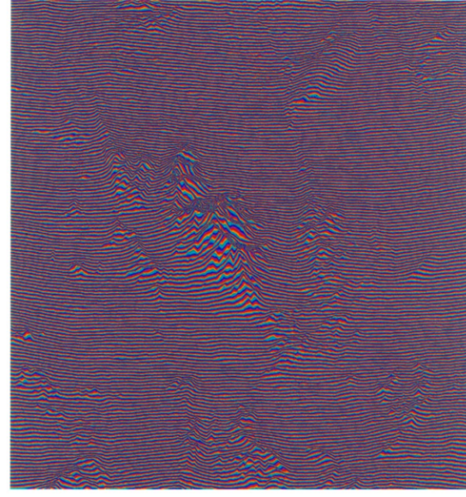

(b)

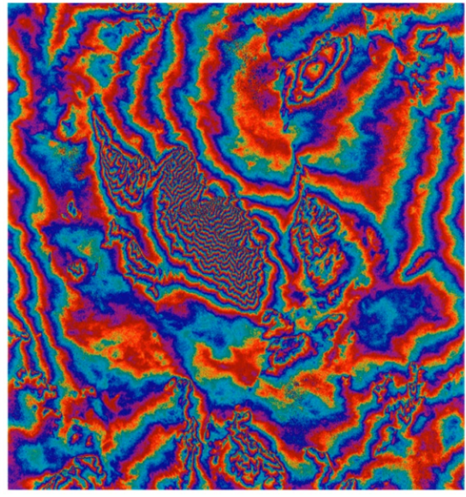

(c)

Figure 3.11.: (a) Amplitude of the master image of a SAR interferometric pair, acquired by the ERS-1/2 satellites at $\mathrm{C}$ band, with $B_{\perp}=133 \mathrm{~m}$, (b) Interferogram before the removal of the flat Earth component, (c) Interferogram after the removal of the flat Earth component, (C)ESA.

where $\Delta R_{\mathrm{m}}$ is the slant range difference between $P_{1}$ and $P_{2}$, depicted in red in Fig. 3.10. $\phi_{\mathrm{fe}}$ has the form of a phase ramp in the slant range dimension and has to be removed from the interferogram, in order to correctly estimate the topographic phase component $\phi_{\text {topo }}$.

As an example, the amplitude image of an interferometric SAR acquisition, acquired by the ERS-1/2 C-band satellites with a normal baseline $B_{\perp}$ of $133 \mathrm{~m}$, is presented in Fig. 3.11 (a). The corresponding interferograms before and after the removal of the flat Earth phase component are shown in 3.11 (b) and (c).

It is important to note that the interferometric phase difference remains ambiguous within intervals of $2 \pi$ and leads to the presence of fringes within the interferogram.

The altitude difference $h_{\mathrm{amb}}$ corresponding to a phase variation of $2 \pi$ is called height of ambiguity. It is inversely proportional to the perpendicular baseline $B_{\perp}$ and is defined as:

$$
h_{\mathrm{amb}}=\frac{\lambda R \sin \theta}{n B_{\perp}}
$$

where $n=2$ for a monostatic configuration and $n=1$ for a bistatic one.

In principle, the higher the perpendicular baseline is, the lower the height of ambiguity is and, as a consequence, the more accurate the height estimation will be. 


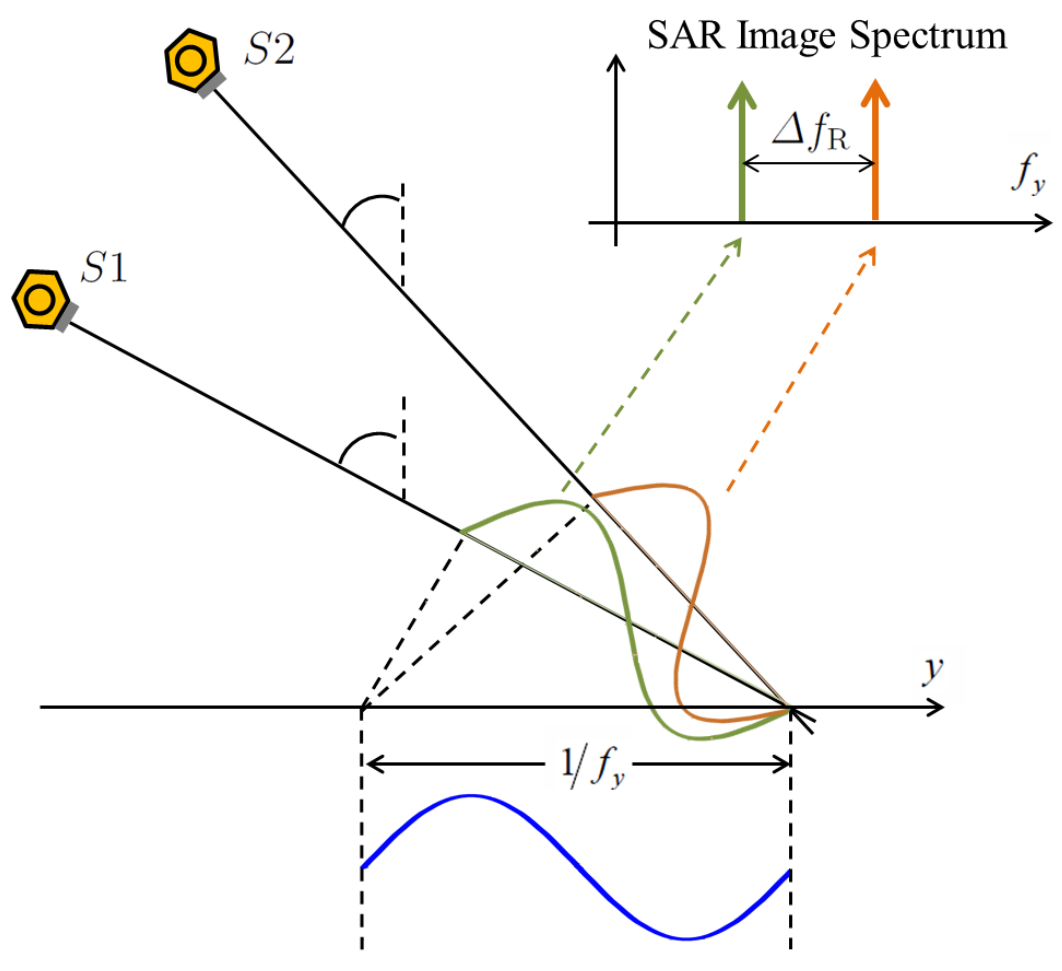

Figure 3.12.: Geometric principle of range spectral shift. Depending on the viewing angle, a ground range sinusoidal frequency component is mapped into two different SAR signal frequencies in the slant range dimension.

\subsubsection{Spectral Shift}

During an interferometric acquisition, a target on ground is typically illuminated under two different look angles by the $S 1$ and $S 2$ sensors, respectively. This causes a misalignment of the recorded signal spectra called spectral shift.

The geometrical interpretation of this phenomenon is simplified in Fig. 3.12, where it can be seen how a ground range frequency component (a sinusoid, in this case) is mapped into two different SAR signal frequencies in the slant range dimension, depending on the illumination angle. This results in a mutual shift $\Delta f_{\mathrm{R}}$ between the two acquired SAR images spectra. Interferometry can therefore be carried out only within the common ground frequency band, which can be accomplished by filtering both images within the same bandwidth in the slant range dimension.

Moreover, spectral shift sets an upper bound to $B_{\perp}$, above which the range spectra of the interferometric images pair $\left(u_{1}\right.$ and $\left.u_{2}\right)$ do not overlap with each other. Such a boundary is called critical baseline $B_{\perp, c}$ and can be derived as [52]: 


$$
B_{\perp, c}=\frac{2 B_{\mathrm{rg}} \lambda R \tan (\theta-\beta)}{v_{\mathrm{lux}}} .
$$

In this case, $u_{1}$ and $u_{2}$ are completely decorrelated and no interferogram can be generated at all.

\subsubsection{The interferometric Coherence}

The interferometric coherence $\gamma_{\text {Tot }}$ is the key parameter for measuring the performance of an interferogram [52]. It represents the correlation coefficient between the two complex SAR images $u_{1}$ and $u_{2}$ and is given by:

$$
\gamma_{\mathrm{Tot}}=\frac{E\left\{u_{1} u_{2}^{*}\right\}}{\sqrt{E\left\{\left|u_{1}\right|^{2}\right\} E\left\{\left|u_{2}^{*}\right|^{2}\right\}}},
$$

where the symbol $*$ identifies the complex conjugate. In reality, the interferometric coherence is estimated from the real SAR data by applying a small window $W$ centered around the considered pixel of indices $[i, j]$ :

$$
\left|\hat{\gamma}_{\text {Tot }}[i, j]\right|=\frac{\left|\sum_{W} u_{1}[i, j] u_{2}^{*}[i, j]\right|}{\sqrt{\sum_{W}\left|u_{1}[i, j]\right|^{2} \sum_{W}\left|u_{2}^{*}[i, j]\right|^{2}}} .
$$

As presented in [25], $\gamma_{\text {Tot }}$ can be theoretically decomposed into different contributions as:

$$
\gamma_{\text {Tot }}=\gamma_{\mathrm{SNR}} \gamma_{\text {Quant }} \gamma_{\mathrm{Amb}} \gamma_{\mathrm{Rg}} \gamma_{\mathrm{Az}} \gamma_{\mathrm{Vol}} \gamma_{\mathrm{Temp}}
$$

where the different terms on the right-hand side of (3.48) identify the correlation factors due to limited SNR $\left(\gamma_{\mathrm{SNR}}\right)$, quantization $\left(\gamma_{\text {Quant }}\right)$, ambiguities $\left(\gamma_{\mathrm{Amb}}\right)$, baseline decorrelation $\left(\gamma_{\mathrm{Rg}}\right)$, relative shift of the Doppler spectra $\left(\gamma_{\mathrm{Az}}\right)$, volume decorrelation $\left(\gamma_{\mathrm{Vol}}\right)$, and temporal decorrelation $\left(\gamma_{\text {Temp }}\right)$.

The lower the coherence is, the noisier the interferogram and, therefore, the less reliable the final DEM will be. An example of the interferometric coherence from TanDEM-X data is depicted in Fig. 3.14 (c).

\subsubsection{Phase Unwrapping and DEM Generation}

As already mentioned in section 3.2.2, the interferometric phase is measured modulo $2 \pi$. Therefore, to reconstruct the topographic height from the interferogram, it is necessary to convert the wrapped interferometric phase $\phi_{\text {int }}$ into an absolute phase $\phi_{\text {unw }}$, by adding to each fringe the correct multiple of $2 \pi$. Such process is called phase unwrapping and its principle is presented in Fig. 3.13. 
(a)
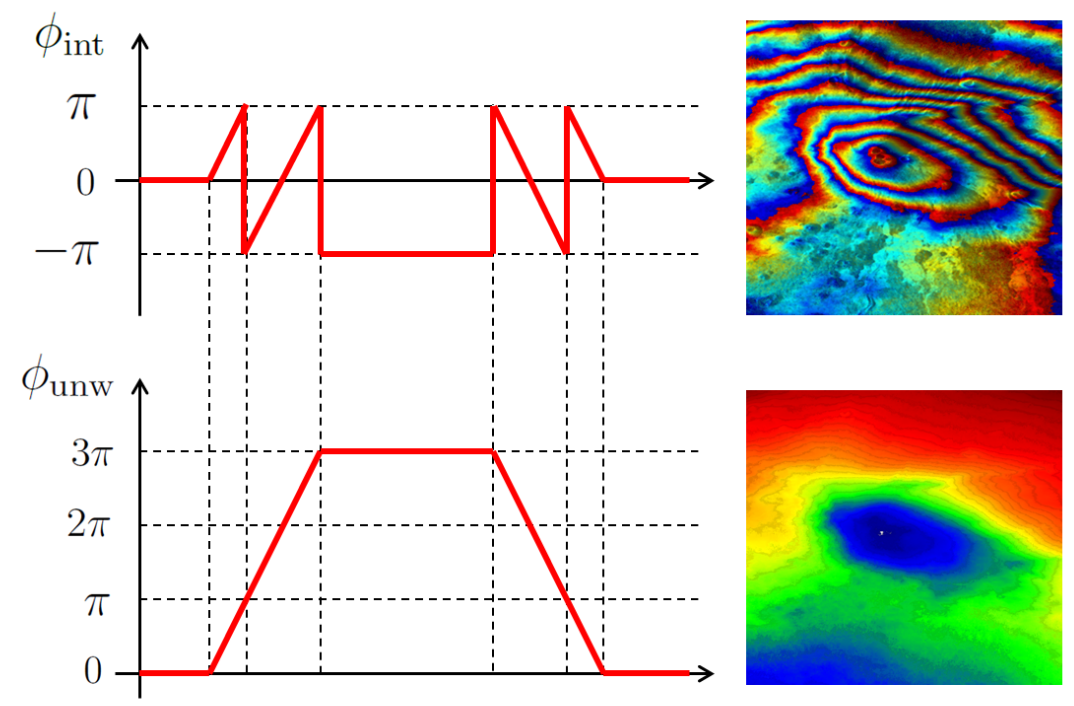

Figure 3.13.: Principle of phase unwrapping. (a) Interferometric phase $\phi_{\text {int }}$, wrapped within $[-\pi, \pi]$, (b) Corresponding unwrapped absolute phase $\phi_{\text {unw }}$. The two images on the right-hand side are taken from a repeat-pass SIR-C/XSAR interferometric acquisition over Mount Etna, Italy.

Several complex algorithms for solving the problem have been developed throughout the years. In the field of SAR interferometry, the issue was first introduced by Goldstein et al. in 1988, who introduced the concept of inconsistencies, called residues, in the wrapped interferometric phase [53]. The basic idea relies on the fact that joining residues with curves, which cannot be crossed during the phase integration of nearby pixels, allows to derive a consistent unwrapped phase.

Further developments in phase unwrapping can be found in [54], [55], [56], and [57]. The unwrapped phase from the interferogram finally allows for the estimation of the ground topography as presented in section 3.2.1.

A final summarizing example is presented in Fig. 3.14, where a TanDEM-X acquisition over British Columbia, Canada is considered. Here, the four main results from the entire interferometric processing are displayed: from the SAR amplitude image, through the generation of the interferogram and of the interferometric coherence, up to the final geocoded DEM. 


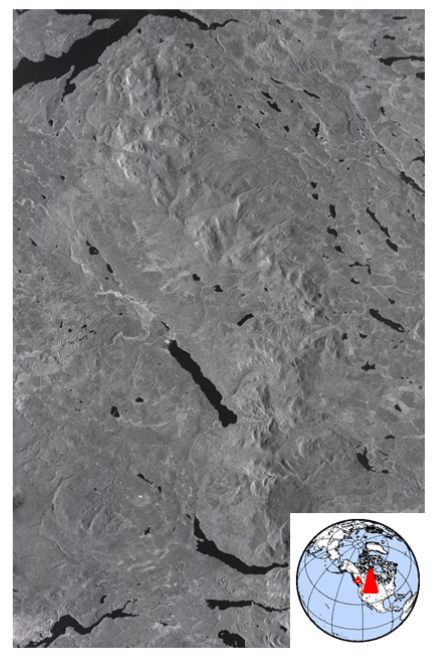

(a)

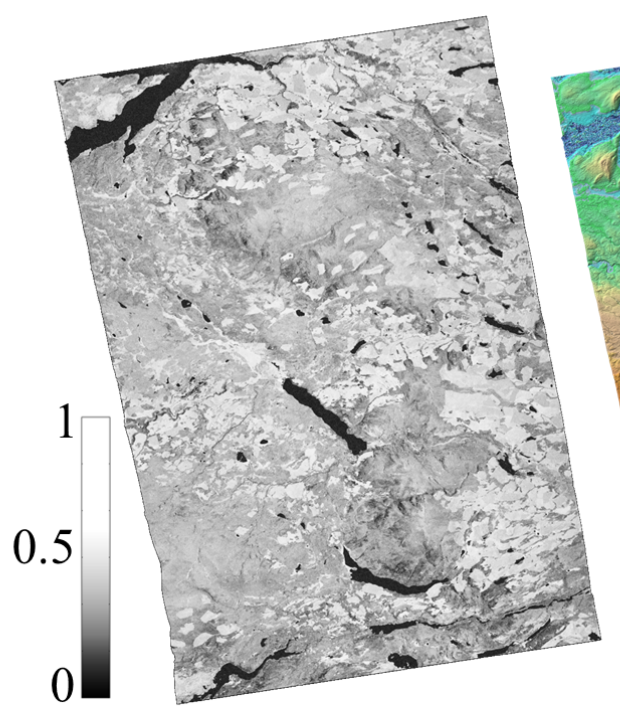

(c)

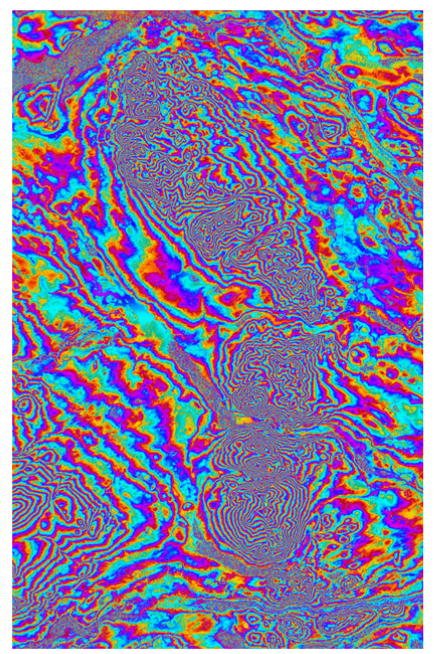

(b)

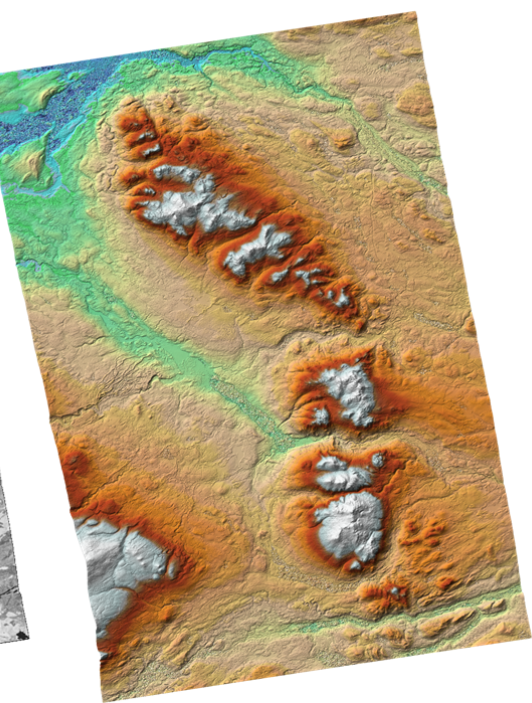

(d)

Figure 3.14.: (a) Amplitude image in SAR coordinates of a TanDEM-X acquisition over British Columbia (Canada), acquired with a perpendicular baseline $B_{\perp}$ of $146 \mathrm{~m}$. (b) Corresponding interferogram after the removal of the flat Earth phase component. (c) Geocoded Image of the interferometric coherence. (d) Geocoded DEM. 


\subsubsection{Chapter Remarks}

In this chapter, the basic concepts of SAR image formation and SAR interferometry have been presented.

One of the novel contributions of the thesis, presented in chapter 5, aims at modeling radar backscatter from detected SAR images. For this reason, the reader has now been provided with a short description of all main steps, which lead to the generation of a SAR image, starting from the acquisition geometry, up to range and azimuth focusing, absolute calibration, and geocoding.

Additionally, several key aspects of SAR images, whose knowledge contributes to a better understanding of backscatter properties, have been discussed as well. For example, in presence of high-relief terrain, geometric distortions, such as shadow and layover, result from the typical side-looking geometry of SAR systems and strongly affect radar backscatter levels. Moreover, image speckle, caused by the coherent sum of scattering contributions within a single resolution cell, results in a random pixel-to-pixel intensity fluctuation, which can be mitigated with multilooking.

In the second part of the chapter, the basic concepts of SAR interferometry have been introduced. Here, the main steps for generating a DEM from a pair of interferometric SAR acquisitions have been presented, including the computation of the interferogram, the effects of spectral shift, and the final phase unwrapping.

Moreover, the key parameter for assessing the performance of an interferogram, namely the interferometric coherence, has been defined as well.

The reader should keep in mind all the mentioned aspects, especially for the understanding of the work presented later on in chapter 6 , where different snow facies of the Greenland Ice Sheet are located my means of interferometric TanDEM-X data. 


\section{The TanDEM-X Mission and its Global Data Set}

In this chapter a brief overview of the spaceborne TanDEM-X mission is presented, focusing on its goals and on the description of the provided data set of quicklook images. The latter serves as input for the application of the developed algorithm for modeling backscatter, described in chapter 5 .

\subsection{TerraSAR-X and TanDEM-X Satellites}

TerraSAR-X (TSX) and TanDEM-X (TDX), launched in June 2007 and June 2010, respectively, are two German SAR satellites, developed within a public/private partnership between the German Aerospace Center (DLR) and Airbus Defence and Space. Both satellites are nearly identical and serve two different SAR missions: the TerraSAR-X mission [58], where both satellites provide high-quality SAR products for both the scientific community and commercial purposes, and the TanDEM-X mission [25], which has the primary goal of generating a global, high precision, and consistent DEM [59]. An artistic graphic of the two satellites in formation flight is shown in Fig. 4.1. Both of them are equipped with a phased array synthetic aperture radar antenna operating at $\mathrm{X}$ band, with a center frequency of $9.65 \mathrm{GHz}$, and can operate in different imaging modes: stripMap, scanSAR, Spotlight, staring Spotlight, and experimental TopSAR. Additionally, by exploiting the unique design of such antennas, single, dual, and even full polarimetric data takes can be performed. The main system parameters, together with ground resolutions and scene sizes, are summarized in Table 4.1.

Dedicated calibration campaigns have been performed during the commissioning phases in order to precisely calibrate both satellites, using transponders and corner reflectors of the DLR SAR calibration facility [60]. The complete performance of the delivered SAR products and of both on-board antennas was also verified during the commission phases and has been constantly monitored ever since. Currently, both satellites show very stable performance and no relevant drifts or changes in their operation has been detected [61]. The TSX and TDX satellites were initially designed for a nominal lifetime of 5.5 years. Based on the current status of system resources, TSX is expected to operate for up to ten additional years, therefore supporting an extended joint coverage with TDX. 


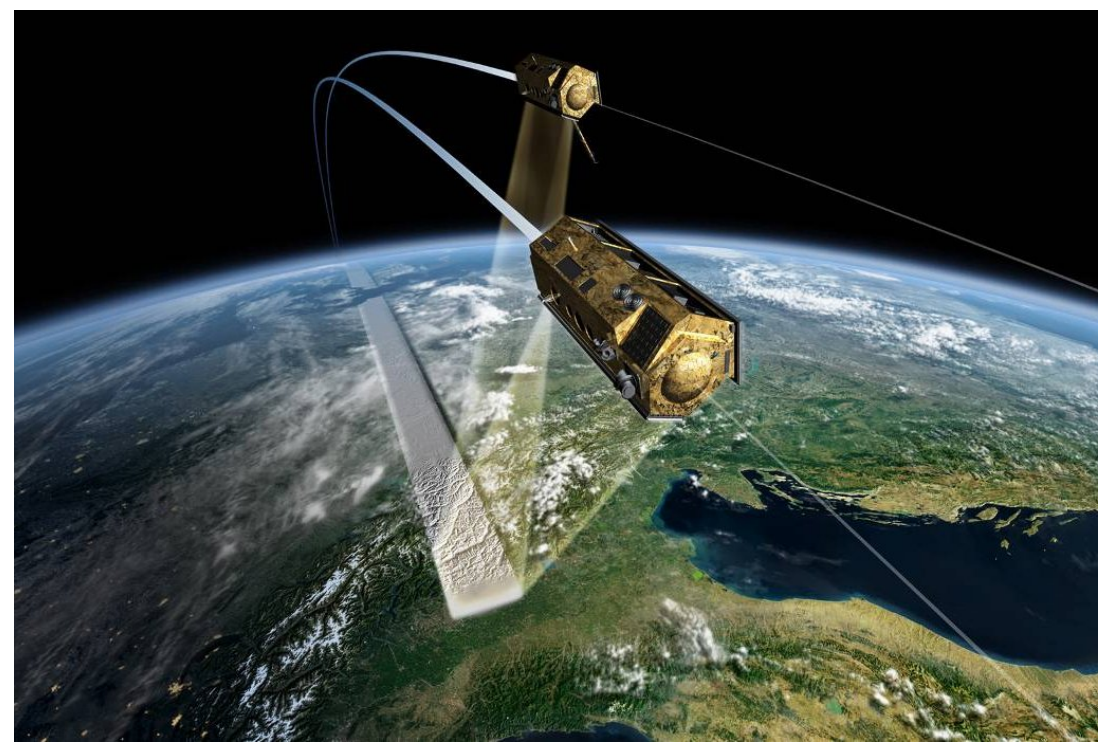

Figure 4.1.: Representation of the TerraSAR-X and TanDEM-X satellites flying in close orbit formation.

Table 4.1.: TerraSAR-X and TanDEM-X System Parameters

\begin{tabular}{cr}
\hline \hline & System Parameters \\
\hline Center frequency & $9.65 \mathrm{GHz}$ \\
\hline Bandwidth & up to $300 \mathrm{MHz}$ \\
\hline Antenna size & $4.8 \mathrm{~m}$ in azimuth, $0.7 \mathrm{~m}$ in elevation \\
\hline Polarization & $\mathrm{H}$ and $\mathrm{V}$ (single, dual, quad) \\
\hline Look angle range & $15^{\circ}-60^{\circ}$ \\
\hline Nominal operation modes & Spotlight, Stripmap, ScanSAR \\
\hline Ground resolution & $0.25 \mathrm{~m}$ (Spotlight) $-40 \mathrm{~m}$ (ScanSAR) \\
\hline Scene size (range) & $10 \mathrm{~km}$ (Spotlight) $-100 \mathrm{Km}$ (ScanSAR) \\
\hline Scene size (azimuth) & $5 \mathrm{Km}$ (Spotlight) $-150 \mathrm{Km} \mathrm{(ScanSAR)}$ \\
\hline Pulse repetition frequency & $2 \mathrm{KHz}-6.5 \mathrm{KHz}$ \\
\hline
\end{tabular}

\subsection{Mission Overview}

The main goal of the TanDEM-X mission is the generation of a worldwide consistent DEM with unprecedented global accuracy. Up to now, DEMs have been derived using several SAR sensors, both airborne and spaceborne. Available global DEMs are for instance the GTOPO3O [62], provided by the United States Geological Survey (USGS) and characterized by a horizontal grid spacing of 30 arc seconds (approximately $1 \mathrm{~km}$ ); the DEM provided by the Shuttle Radar Topography Mission (SRTM) [23], whose coverage is limited to a latitude range between $56^{\circ} S$ and $60^{\circ} \mathrm{N}$ and which is delivered with a grid 


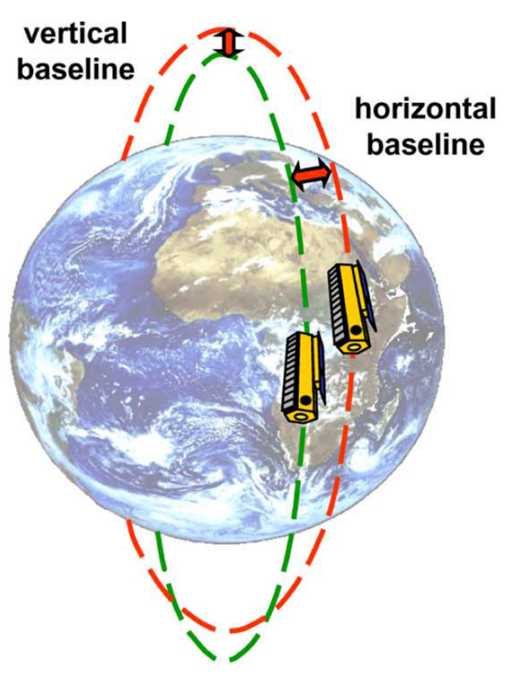

(a)

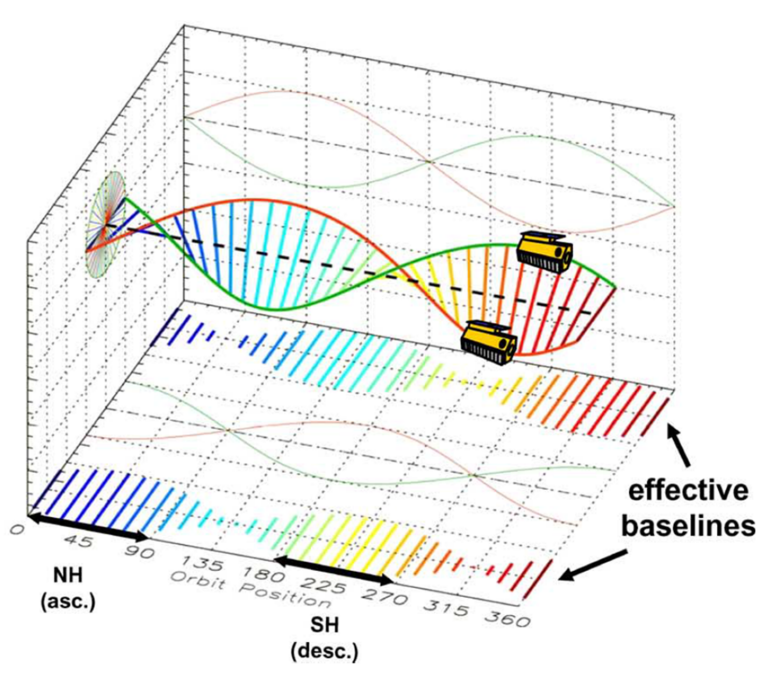

(b)

Figure 4.2.: (a) Orbit illustration of the Helix satellite formation around the Earth and (b) along the orbit direction for TerraSAR-X (red) and TanDEM-X (green) satellites.

spacing of 1 arc second for regions within the U.S. territory and 3 arc seconds for the rest of the mapped areas.

Since October 2010, the TSX and TDX satellites have been flying in a close orbit formation, enabling the acquisition of highly accurate cross- and along-track single-pass interferograms and, subsequently, the generation of high-resolution DEMs. Such a configuration avoids the limitations of repeat-pass interferometry due to temporal decorrelation and atmospheric disturbance.

Both satellites fly in sun-synchronous dusk-dawn orbits, combined together in the innovative Helix satellite formation, whose principle is shown in Fig. 4.2 [63], [64]. It consists of an horizontal and vertical orbital displacement, resulting in a helix-like relative orbit evolution of the satellites, where the two single orbits never cross. Moreover, free shifts and drifts of the satellites along their orbits are possible, allowing for a safe operation of the spacecrafts and for a flexible selection of along- and across-track baselines, typically varying from about $100 \mathrm{~m}$ up to $500 \mathrm{~m}$.

TanDEM-X interferometric acquisitions can be performed in three different operational modes, as presented in Fig. 4.3: 


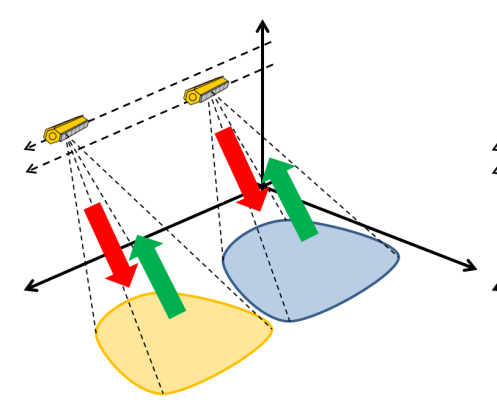

(a)

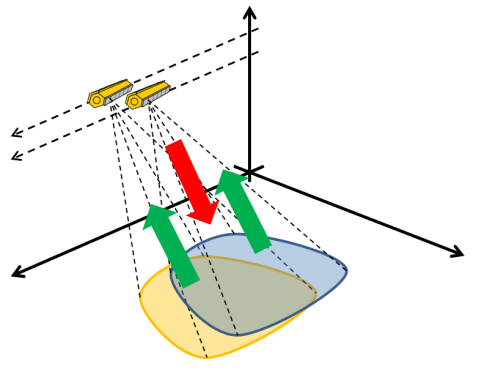

(b)

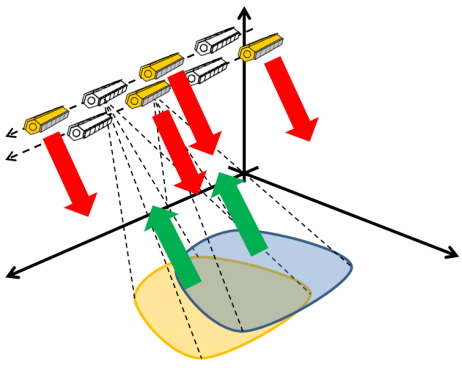

(c)

Figure 4.3.: TanDEM-X mission acquisition modes: (a) Pursuit monostatic, (b) Bistatic, (c) Alternating bistatic.

- Pursuit Monostatic mode: the two satellites operate independently from each other, with no need for precise synchronization.

- Bistatic mode: either TSX or TDX are used as transmitter and both of them receive the signal coming from a common illuminated footprint on ground.

- Alternating Bistatic Mode: The transmitter is switched from pulse to pulse between the two satellites.

All nominal acquisitions for the generation of the global DEM were performed in bistatic configuration.

The generation of the global TanDEM-X DEM has been completed in September 2016 and the global DEM is now available. The main parameters, which have been taken into account for assessing the final performance are explained in the following and their specification is summarized in Table 4.2:

- The DEM spatial resolution describes the minimum distance which allows to correctly discriminate between two different targets. It is specified for both range and azimuth directions and is defined as in (3.2) and (3.7).

- The absolute vertical height accuracy is defined as the uncertainty in the height of a point with respect to the WGS84 ellipsoid caused by random and uncorrected systematic errors. The specified value is expressed as a linear error at the $90 \%$ confidence level at global scale. In case of $X$ band, one should note that elevation data, obtained from SAR interferometry, is defined with respect to the reflective surface 
Table 4.2.: Global TanDEM-X DEM specification.

\begin{tabular}{ccc}
\hline \hline Requirement & Description & Specification \\
\hline Relative Vertical Accuracy & $\begin{array}{c}90 \% \text { linear point-to-point error } \\
\text { over a } 1^{\circ} \text { x } 1^{\circ} \text { cell }\end{array}$ & $\begin{array}{c}2 \mathrm{~m}(\text { slope }<20 \%) \\
4 \mathrm{~m}(\text { slope }>20 \%)\end{array}$ \\
\hline Absolute Vertical Accuracy & $90 \%$ linear error & $10 \mathrm{~m}$ \\
\hline Horizontal Accuracy & $90 \%$ circular error & $3 \mathrm{~m}$ \\
\hline Spatial Resolution & independent pixels & $12 \mathrm{~m}$ \\
& Land masses $\%$ & $3 \%$
\end{tabular}

of X-band SAR returns from on-ground targets. This process locates the position of the mean phase center from multiple backscattered signals within the same resolution cell. Hence, the data includes vegetation canopy elevations, seasonal variations, and man-made structures. The absolute vertical accuracy is specified to be $\leq 10 \mathrm{~m}$ and reference information (e.g. tie points) may be used where necessary to achieve the required accuracy.

- The relative vertical height accuracy is defined as the uncertainty in height between two points caused by random errors. The specified values are expressed as linear errors at a $90 \%$ confidence interval. The relative vertical accuracy shall be better than $2 \mathrm{~m}$ for low and medium relief terrain ( 0 to $20 \%$ predominant slope) and $4 \mathrm{~m}$ for high relief terrain (higher than $20 \%$ predominant slope), over a geocell of $1^{\circ} \times 1^{\circ}$ in latitude/longitude coordinates.

- The horizontal accuracy is defined as the uncertainty in the horizontal position of a point with respect to WGS84 caused by random and uncorrected systematic errors. The value is expressed as a circular error at the $90 \%$ confidence level. The absolute horizontal accuracy shall be $\leq 10 \mathrm{~m}$.

- Voids or Invalid Data are pixels in the DEM that have no data and occur for many reasons, including poor SNR return signal from sandy desert areas or shadow and layover in mountains and canyons, DEM Gaps which result from missing suitable input DEMs to be mosaicked. The specification states that at least $97 \%$ of all DEM pixels over land-covered areas (water bodies excluded) must not be flagged as invalid. Differently from the relative vertical accuracy, the percentage of voids is evaluated on a global scale and not on a geocell basis.

The acquisition strategy has been monitored and optimized during the entire mission in order to satisfy the specification. All land masses have been acquired at least twice and 


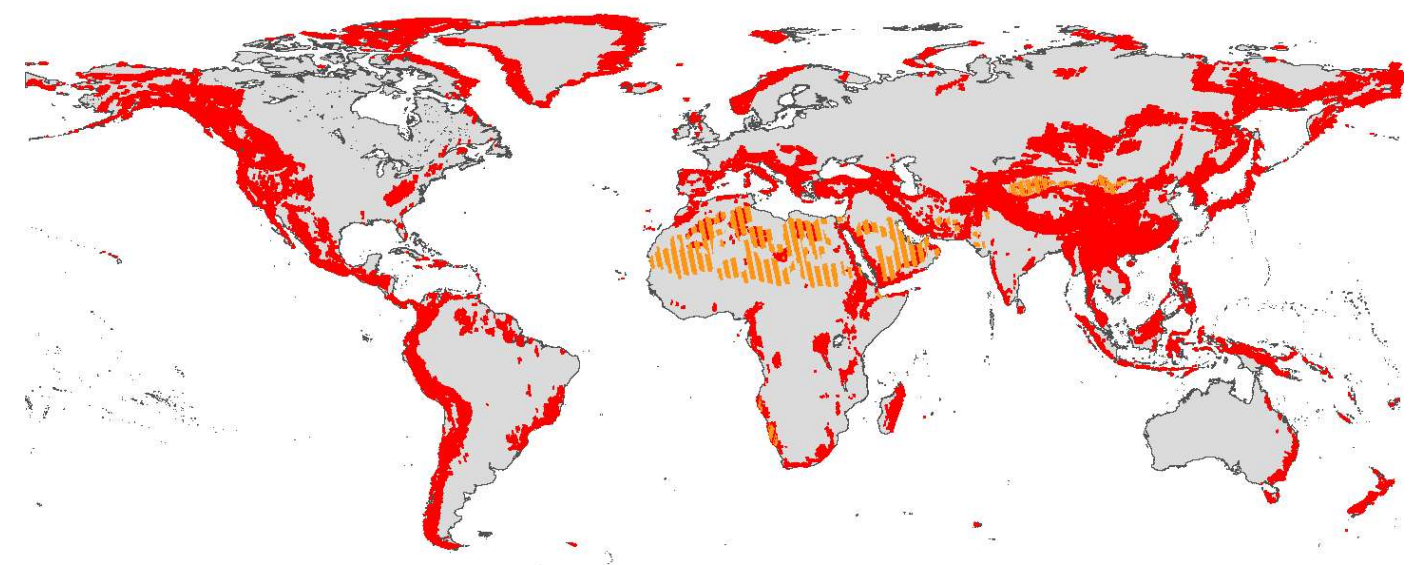

Figure 4.4.: Critical regions affected by shadow and layover (mountains - red) and by low SNR (sandy deserts - orange), which needed to be acquired using optimized geometries for improving the final performance of the global TanDEM-X DEM.

dedicated additional acquisitions have been performed with optimized geometry over critical areas [65]. For example, crossing-orbit acquisitions (acquired in ascending and descending orbit over the same area on ground) have been performed over mountainous areas, in order to correctly fill gaps caused by shadow and layover. Moreover, optimized acquisitions with steep incidence angles have been performed over sandy desert areas, in order to mitigate the effects of low backscatter [66], [67]. Such areas are visible in Fig. 4.4 .

The global performance of the TanDEM-X DEM has been continuously verified during and at the end of the DEM generation process. As exhaustively presented in [68], the mission specification has been fully fulfilled. The global TanDEM-X DEM is finally depicted in Fig. 4.5.

The analysis of the global unedited TanDEM-X DEM product therefore confirms its stunning accuracy and global consistency and is now ready to be put forward as a new global topographic reference and be delivered to both the commercial and scientific communities. Moreover, the TanDEM-X data set represents a highly valuable starting point for monitoring the dynamic topographic changes of our planet.

Beside the generation of the global DEM and thanks to its unique configuration, the TanDEM-X mission allows for the demonstration of innovative bistatic SAR techniques, such as multistatic SAR [69], polarimetry [70] [71], interferometry [72] [73] [74], digital beam forming [75], and superresolution [76], and represents a milestone for the develop- 


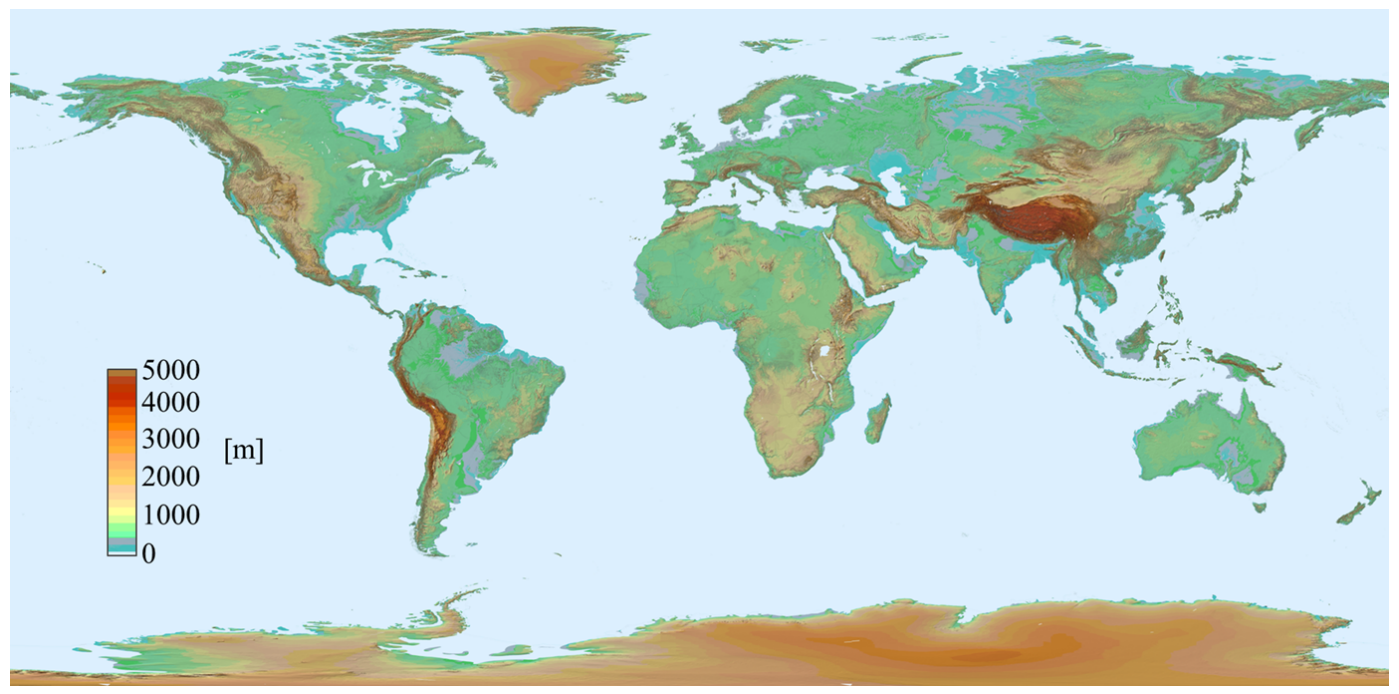

Figure 4.5.: The TanDEM-X global DEM.

ment of future spaceborne formation-flying SAR missions, such as Tandem-L: an L-band bistatic SAR mission proposed by DLR, with the goal of monitoring dynamic processes of the Earth's with unprecedented temporal coverage [77].

\subsection{TanDEM-X Data: Quicklook Images}

Due to processing constraints, TanDEM-X bistatic acquisitions are split into single scenes by the operational Interferometric TanDEM-X Processor (ITP), covering an area on ground of about $30 \mathrm{~km} \times 50 \mathrm{~km}$ in range and azimuth dimensions, respectively. Several quicklooks are delivered as by-products from the interferometric processing chain, including:

- Detected amplitude SAR image in SAR coordinates, which can be absolutely calibrated according to the TSX and TDX absolute calibration constant as in (3.25),

- Geocoded and roughly calibrated DEM (rawDEM), absolutely calibrated using radargrammetry [78],

- Interferometric coherence,

- Incidence angle,

- Georeferencing and geocoding information,

- Meta data (acquisition parameters). 
These quicklooks are obtained by applying a spatial averaging process to the corresponding operational TanDEM-X full-resolution $12 \mathrm{~m} \times 12 \mathrm{~m}$ images. The results are images characterized by a ground pixel spacing of about $50 \mathrm{~m} \times 50 \mathrm{~m}$. All the considered images for the current work were acquired in bistatic stripmap mode and single horizontal polarization $(\mathrm{HH})$, covering the entire range of incidence angles of the nominal TanDEM-X mission, between $29.8^{\circ}$ and $45.8^{\circ}$.

Regarding the detected amplitude SAR quicklook image, a geocoded $\beta^{0}$ image is evaluated from the detected and absolutely calibrated amplitude master image, acquired by the active satellite. Both TSX and TDX can alternatively act as the active sensor, which means transmit and receive radar pulses (the passive one receives only), leading to the generation of an image in monostatic configuration. Since both satellites show an almost identical behavior in terms of calibration [79] and system performance [61], either TSX and TDX are indistinctively used as active satellite for the current analysis of radar backscatter.

Because of computational costs in time, geocoding is obtained by bilinearly interpolating the detected and absolutely calibrated input image in SAR coordinates on a coarse mapping grid [80], available within each quicklook product itself, which correlates pixels of the geocoded image with range/azimuth times. Such an interpolation can introduce geolocation errors, due to the lower resolution of the geocoding grid with respect to the one of the input amplitude image. A more detailed quantification of such errors is given in section 5.3.4.

Several other quicklook images can be derived from the ones described above, among which:

- Signal-to-noise ratio (SNR), evaluated as:

$$
\mathrm{SNR}=\frac{\beta^{0} \sin \theta-\mathrm{NESZ}}{\mathrm{NESZ}},
$$

being NESZ the noise equivalent sigma zero, which represents the system noise floor. Fig. 4.6 (a) depicts the calculated NESZ profiles for all different operational TanDEM-X beams, evaluated as in [25].

- SNR correlation factor, identified a $\gamma_{\mathrm{SNR}}$ in (3.48):

$$
\gamma_{\mathrm{SNR}}=1 /\left(1+\mathrm{SNR}^{-1}\right)
$$

where SNR is is assumed to be equal in both monostatic and bistatic images. 

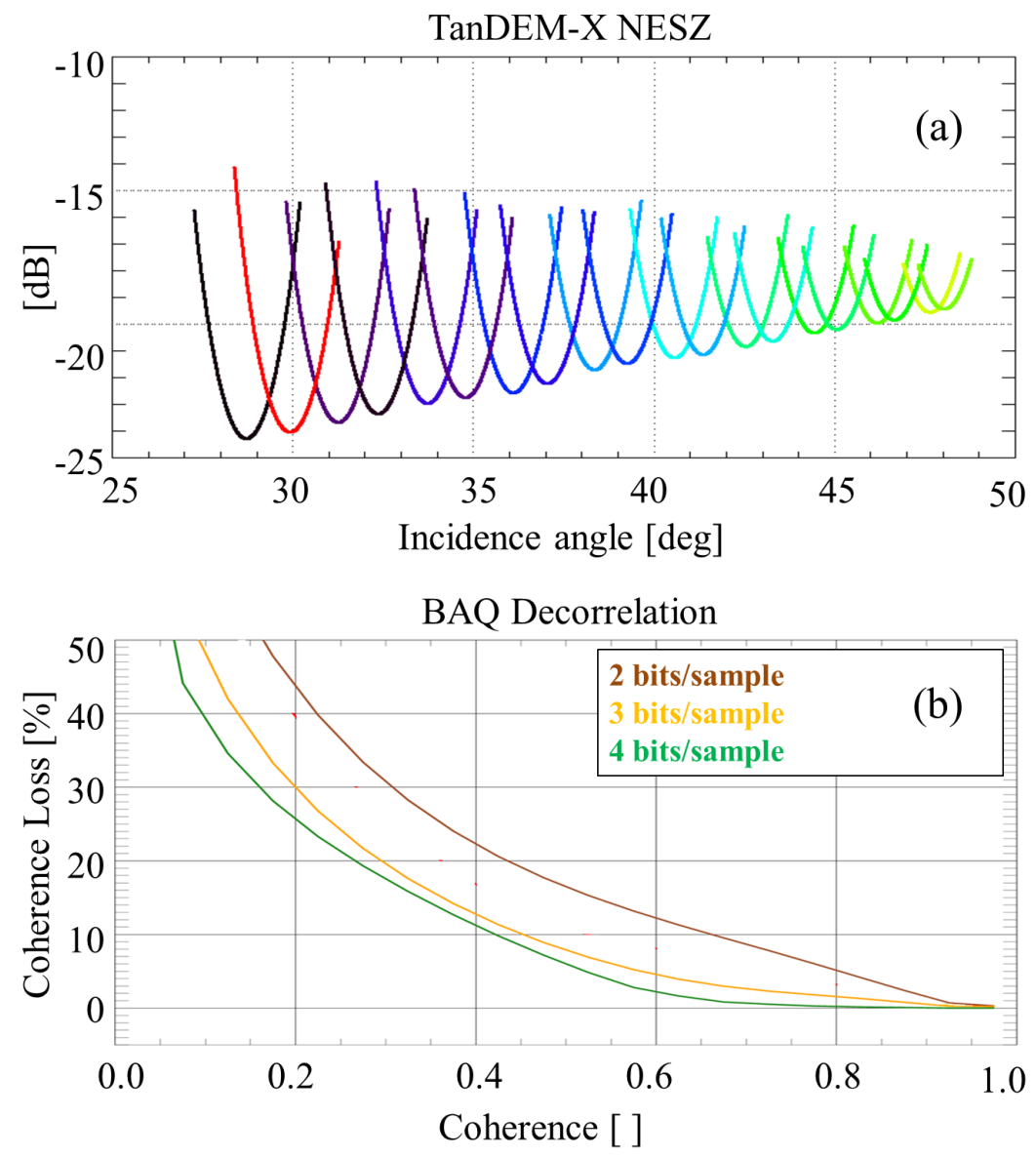

Figure 4.6.: (a) NESZ (Noise Equivalent Sigma Zero) profiles for the operational TanDEM-X beams [25]. (b) Coherence loss due to BAQ (Block Adaptive Quantization). Each curve identifies a different quantization rate (2, 3, and 4 bits/sample).

- Quantization correlation factor $\gamma_{\text {Quant }}$, found by interpolating the look-up-table functions in Fig. 4.6 (b), which is derived from real TanDEM-X data [81]. Block adaptive quantization (BAQ) has been used [82].

- The coherence contribution due to volume decorrelation, called volume correlation factor $\gamma_{\mathrm{Vol}}$, can be estimated from the total interferometric coherence by inverting (3.48) as:

$$
\gamma_{\text {Vol }}=\frac{\gamma_{\text {Tot }}}{\gamma_{\text {SNR }} \gamma_{\text {Quant }} \gamma_{\text {Amb }} \gamma_{\text {Rg }} \gamma_{\text {Az }} \gamma_{\text {Temp }}}
$$

where, according to the performance estimation analysis for TanDEM-X [25], $\gamma_{\mathrm{Amb}}, \gamma_{\mathrm{Rg}}$, and $\gamma_{\mathrm{Az}}$ are assumed to introduce a further overall correlation factor 
of $0.98(2 \%)$, while $\gamma_{\text {Temp }} \simeq 1$, since images are acquired in bistatic configuration.

- A map of the predominant local slope can be derived starting from the available rawDEM. Firstly, such a DEM, expressed in latitude/longitude (lat/lon) reference coordinates, has to be converted into horizontal and vertical spatial distances, $x$ and $y$, by evaluating the distance between adjacent pixels on the ellipsoid, leading to:

$$
\operatorname{DEM}(\text { lat, lon }) \underset{\mathrm{WGS} 84}{\Rightarrow} D(x, y) \text {. }
$$

Secondly, the bi-dimensional local gradient of $D(x, y)$, evaluated as:

$$
\nabla D(x, y)
$$

can be decomposed into its horizontal and vertical components as:

$$
\nabla D(x, y)=\left(\frac{\partial D(x, y)}{\partial x}, \frac{\partial D(x, y)}{\partial y}\right)
$$

The predominant local slope $\Lambda(x, y)$ is then retrieved by computing the Euclidean norm of the gradient vector as:

$$
\Lambda(x, y)=\sqrt{\left(\frac{\partial D(x, y)}{\partial x}\right)^{2}+\left(\frac{\partial D(x, y)}{\partial y}\right)^{2}} .
$$

A summarizing example of geocoded TanDEM-X quicklook images is finally presented in Fig. 4.7.

A crucial aspect is to verify that multilooking processing does not significantly impact the backscatter statistics, which, would lead to biased estimations. The verification of the derived backscatter quicklook images has been performed prior to the development of the modeling algorithm, presented later on in chapter 5 , by comparing a finite set of test images to the corresponding interferometric products at $12 \mathrm{~m} \times 12 \mathrm{~m}$ resolution (CoSSC).

An example is presented in Fig. 4.8 (a) for a TanDEM-X acquisition over the Amazon rain forest. The normalized histograms of $\gamma^{0}$ are shown in Fig. 4.8 (b). As expected, the multilooking process, applied to quicklook images, leads to a compression of the overall $\gamma^{0}$ dynamics. However, this process does not significantly impact the $\gamma^{0}$ mean value, as can be inferred from Fig. 4.8 (c), where the mean $\gamma^{0}$ slant range profile is depicted. Considering the entire image, the following mean $\gamma^{0}$ values are obtained: $\mathrm{E}\left[\gamma^{0}\right]_{\operatorname{coSSC}}=-6.82 \mathrm{~dB}$ and $\mathrm{E}\left[\gamma^{0}\right]_{\text {quicklook }}=-6.83 \mathrm{~dB}$ for the coSSC and quicklook 


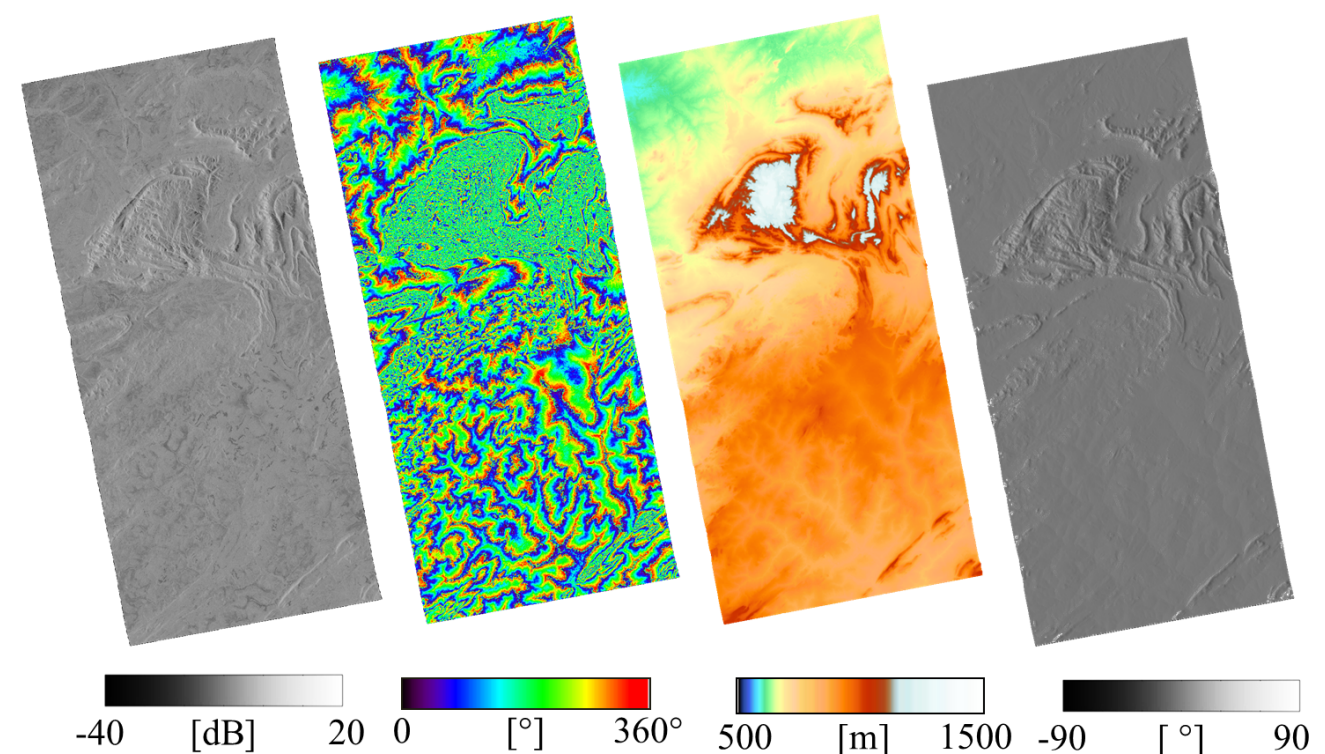

(a)

(b)

(c)

(d)

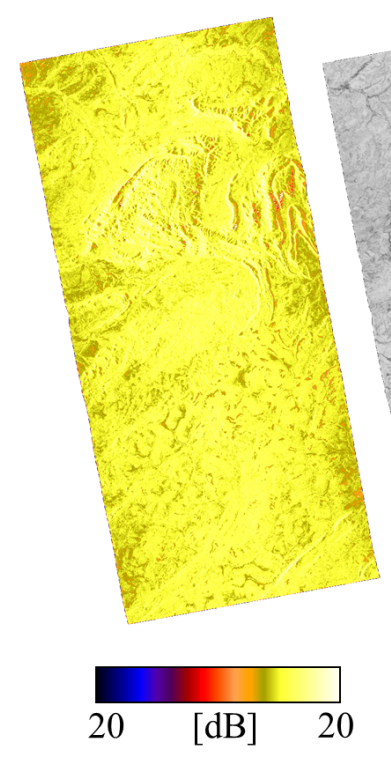

(e)

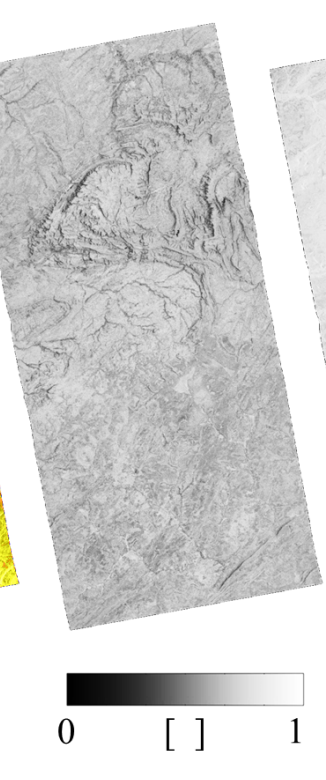

(f)

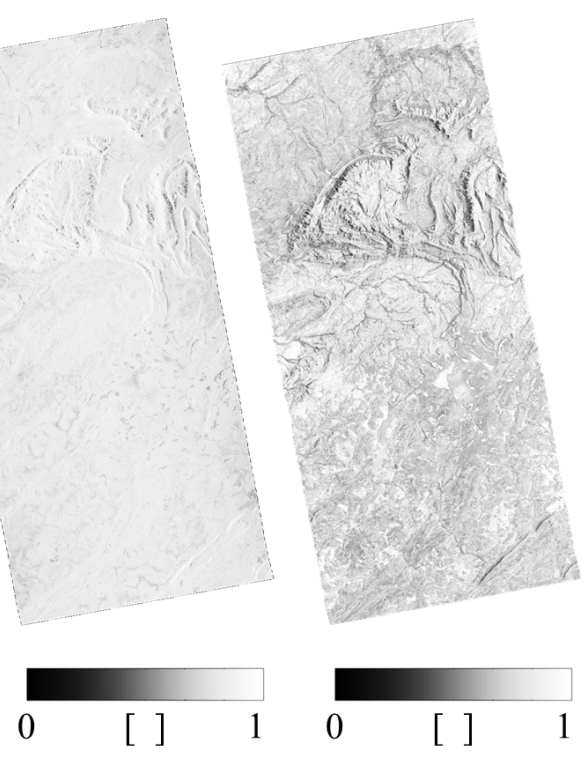

(g)

(h)

Figure 4.7.: Example of TanDEM-X geocoded quicklooks associated to a single processed scene. (a) Backscatter $\beta^{0}$, (b) Interferogram, (c) DEM, (d) Predominant local slope, (e) SNR, (f) Interferometric coherence $\gamma_{\text {Tot }}$, (g) SNR correlation factor $\gamma_{\mathrm{SNR}}$, (h) Volume correlation factor $\gamma_{\mathrm{Vol}}$. 

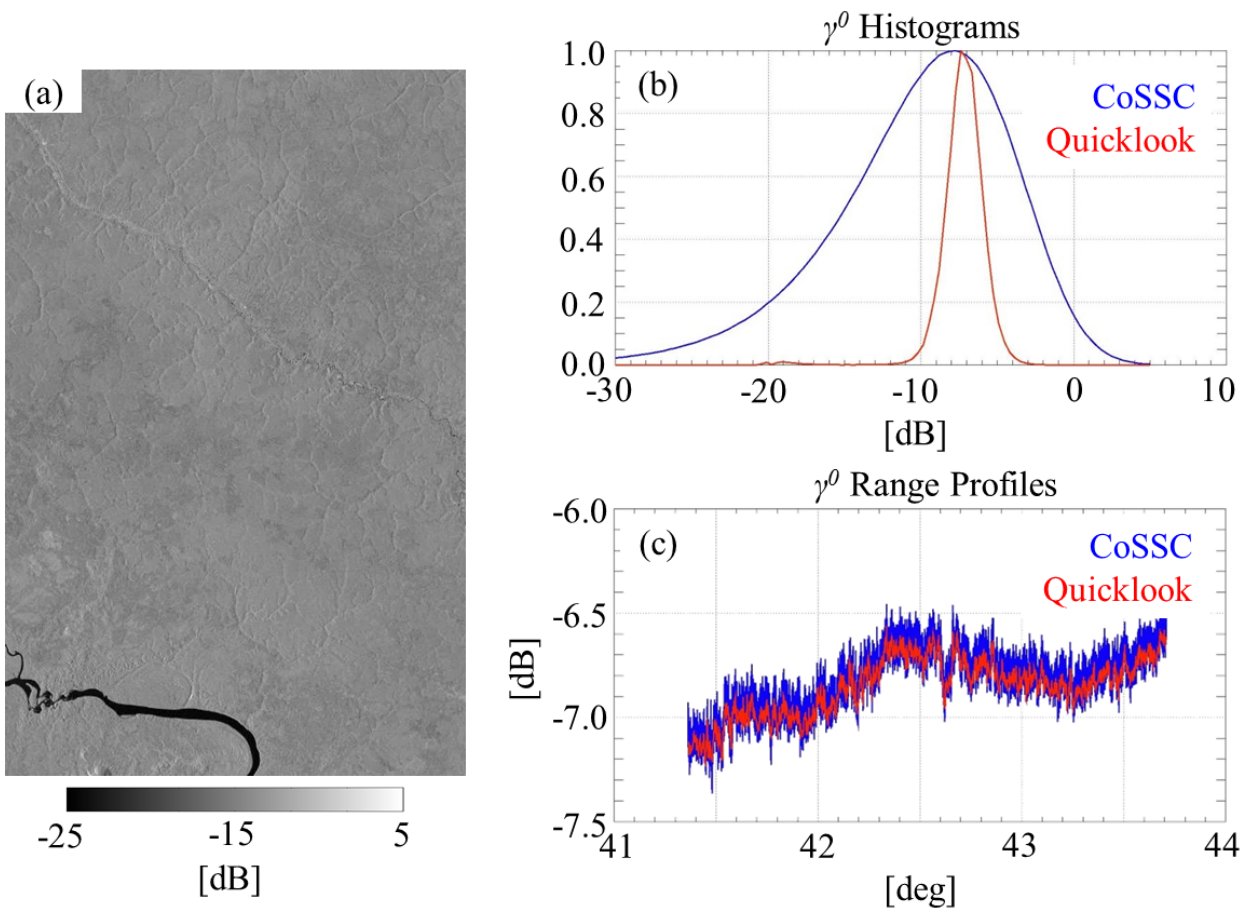

Figure 4.8.: (a) $\gamma^{0}$ image of a TanDEM-X acquisition over the Amazon Rain forest. (b) Corresponding normalized $\gamma^{0}$ histograms from the CoSSC $(12 \mathrm{~m} \times 12 \mathrm{~m}$ resolution, blue) and quicklook (50 $\mathrm{m} \times 50 \mathrm{~m}$ resolution, red) products, respectively. (c) Mean $\gamma^{0}$ range profiles for the CoSSC (blue) and quicklook (red) products; the horizontal axis displays the incidence angle in degrees.

image, respectively. Both products match together well, showing a negligible mean $\gamma^{0}$ difference of just $0.01 \mathrm{~dB}$.

The choice of using quicklooks instead of full-resolution products resides in the necessity of reducing the computational costs when working at a global scale. The Integrated TanDEM-X Processor (ITP) has delivered more than 500,000 scenes as input data base for the global DEM. As an example, a full resolution map of the the interferometric coherence is generated for each scene by the ITP as well. Each coherence map has a size of about 350 megabytes per scene, leading to an overall data volume in the order of 175 terabytes. A quicklook image of the interferometric coherence for a single TanDEM-X scene typically has a size of about 0.8 megabytes. The overall amount of quicklook data that has to be managed is therefore in the order of 0.5 terabytes. Hence, for a single scene, the use of quicklook images at a global scale instead of full-resolution products allows for reducing the overall data volume by about 400 times. 


\section{A New Approach for Statistical Modeling of Radar Backscatter}

In this chapter, a novel approach, developed to model radar backscatter from SAR acquisitions, is presented. It is based on a statistical analysis of backscatter properties, depending on several parameters such as the acquisition geometry and the type of land cover. Such an approach has been developed using TanDEM-X data as reference, but can be generalized for a theoretical SAR acquisition scenario.

The main content of the work presented in this chapter was published in [83].

\subsection{State of the Art in Radar Backscatter Modeling}

A precise knowledge of the radar backscatter properties, depending on different parameters, such as acquisition geometry, ground vegetation, seasonal time, and meteorological conditions, represents a powerful mean in many branches of remote sensing. Several models and modeling techniques have been developed in the past for characterizing backscatter behavior, depending on the described parameters.

The comprehensive database provided by F. T. Ulaby and M. C. Dobson in 1989 is widely used within the scientific community. This was generated by composing measurements at different frequencies, derived from field observations using ground scatterometers in North America [38].

Many significant works for modeling radar backscatter at a local scale take into account a specific kind of on ground cover type and find application in different research fields.

For example, radar backscatter modeling of forest canopies is a highly interesting topic and finds application e. g. in the estimation of the biomass. One of the first works about forest backscatter modeling was provided by Richards et al. in [84]: they derived a Lband $\mathrm{HH}$ radar backscatter model of a coniferous forest and compared it with data from the SIR-B L-band images of the Mount Shasta region of northern California. Specular reflections from tree trucks were introduced and backscatter returns were modeled as the sum of canopy and trunk contributions. Relevant works have been published by G. Sun 
and K. Ranson in [85], who modeled backscatter returns from a volumetric vegetated cell using ray-tracing techniques. Martinez et al. in [86] used data from the airborne ranging Helsinki University of Technology Scatterometer (HUTSCAT) to model vertical backscatter profiles within a canopy over an Austrian pine forest in southern France and studied the radar wave penetration into a volume.

Backscatter behavior from Sahara desert sandy dunes was investigated by H. Stephen and D. G. Long in [87], using data from different scatterometers. They were able to observe a characteristic relationship between backscatter returns and dunes topography: small ripples were modeled using cosinusoidal surface waves, while longitudinal and transverse dunes were associated to Gaussian tilt distributions.

Relevant works in the field of glaciology are e.g. the characterization of radar backscatter of ice-covered areas in Greenland by I. Ashcraft and D. Long in [88], carried out by analyzing data from the NASA scatterometer NSCAT and from the C-band ERS satellite, or the backscatter modeling for river ice from I. Gherboudj et al. in [89]. More recently, Parrella et al. investigated L-band polarimetric SAR data from the E-SAR airborne sensor of the German Aerospace Center, associating different scattering models to specific glacier facies [90].

The investigation of X-band radar backscatter from spaceborne platform has started relatively recently, and the interest of the scientific community in such a topic has significantly grown in the last years, given the availability of X-band SAR images for scientific purposes from the TerraSAR-X, TanDEM-X, and COSMO-SkyMed missions.

\subsection{Mapping Radar Backscatter}

Different quantities can be used to represent backscatter from SAR data, as described in [91] and [38]. The backscattering coefficients $\sigma^{0}$ and $\gamma^{0}$, defined in (2.44) and (2.46) as the backscatter cross-section per unit area on ground and per unit area perpendicular to the antenna beam, respectively, are commonly used within the scientific community for the retrieval of bio- or geophysical parameters.

The reflectivity per unit area in slant range, called radar brightness $\beta^{0}$ and introduced in (3.25), is the only quantity which does not require the knowledge of the local incidence angle for its computation [92]. Such an angle varies according to the local slope of the illuminated ground area, as shown in Fig. 2.5. This is one of the main reasons why modern SAR missions, such as TerraSAR-X and TanDEM-X [93], RADARSAT [94], and ENVISAT ASAR [95], deliver their radar products in $\beta^{0}$ format, avoiding inaccurate estimation of local incidence angles due to an approximate terrain knowledge. The 
excellent calibration of the TanDEM-X system assures that the provided $\beta^{0}$ images are not affected by significant systematic errors [79] [96]. Thus, the current derivation of backscatter models is performed by using radar brightness $\beta^{0}$ as a starting point.

Eventually, $\sigma^{0}$ and $\gamma^{0}$ can be directly estimated from the radar brightness by compensating for the sine and the tangent, of the local incidence angle, respectively.

\subsection{A New Method for Modeling Backscatter}

The proposed method for modeling radar backscattering and its verification approach consists of two main steps:

1. A statistical analysis of the backscatter information within a single SAR acquisitions is performed, as explained in section 5.3.1. If repeated over a large number of acquisitions, such an approach allows to build an input data base of backscatter statistics.

2. The data base generated at step 1 represents the input starting point for generating statistical models of the mean backscatter at a global scale, as presented in section 5.3.2.

This technique could be applied to different data sets from several SAR missions. For practical reasons, the examples presented in this section to support the proposed theory are based on the use of SAR data from the TanDEM-X mission, which has been presented in chapter 4.

\subsubsection{Backscatter Characterization from Single SAR Acquisitions}

A statistical approach is used to characterize the mean backscatter behavior within a single SAR acquisition. For each given input acquisition, a geocoded $\beta^{0}(x, y)$ image is evaluated from the detected and absolutely calibrated amplitude image, derived as presented in section 3.1.7, where $x$ and $y$ are the horizontal and vertical coordinates, respectively.

For the current analysis the GlobCover classification map, provided by the European Space Agency (ESA), is used to perform the classification [97]. Nevertheless, other classification maps could be considered as well. GlobCover is composed by 23 different classes (22 valid classes and 1 invalid class), shown in Fig. 5.1. The available classes are summarized in the legend, where a numerical identifier is associated to each of them. The map is characterized by a ground pixel spacing of $300 \mathrm{~m} \times 300 \mathrm{~m}$, projected on the WGS84 ellipsoid. In order to classify each $\beta^{0}(x, y)$ input pixel, a nearest neighbor 


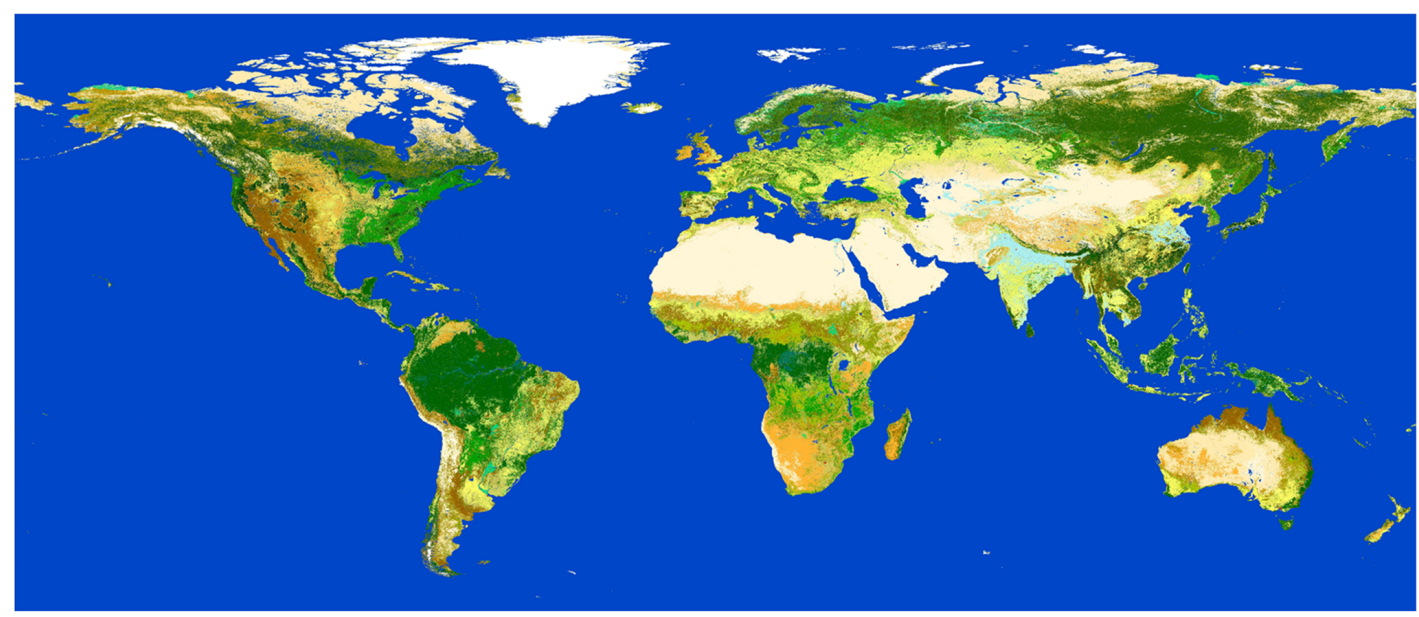

\begin{tabular}{|c|c|}
\hline 1 & Post-flooding or irrigated croplands (or aquatic) \\
\hline 2 & Rainfed croplands \\
\hline 3 & Mosaic cropland (50-70\%) / vegetation (grassland/shrubland/forest) (20-50\%) \\
\hline 4 & Mosaic vegetation (grassland/shrubland/forest) $(50-70 \%) /$ cropland (20-50\%) \\
\hline 5 & Closed to open (gt 15\%) broadleaved evergreen or semi-deciduous forest $(>5 \mathrm{~m})$ \\
\hline 6 & Closed $(>40 \%)$ broadleaved deciduous forest $(>5 \mathrm{~m})$ \\
\hline 7 & Open (15-40\%) broadleaved deciduous forest/woodland $(>5 \mathrm{~m})$ \\
\hline 8 & Closed $(>40 \%)$ needleleaved evergreen forest $(>5 \mathrm{~m})$ \\
\hline 9 & Open $(15-40 \%)$ needleleaved deciduous or evergreen forest $(>5 \mathrm{~m})$ \\
\hline 10 & Closed to open $(>15 \%)$ mixed broadleaved and needleleaved forest $(>5 \mathrm{~m})$ \\
\hline 11 & Mosaic forest or shrubland (50-70\%) / grassland (20-50\%) \\
\hline 12 & Mosaic grassland (50-70\%) / forest or shrubland (20-50\%) \\
\hline 13 & Closed to open $(>15 \%)$ (broadleaved or needleleaved) shrubland $(<5 \mathrm{~m})$ \\
\hline 14 & Closed to open (>15\%) herbaceous vegetation (grassland, savannas or lichens/mosses) \\
\hline 15 & Sparse $(<15 \%)$ vegetation \\
\hline 16 & Close to open $(>15 \%)$ broadleaved forest regularly flooded \\
\hline 17 & $\begin{array}{l}\text { Closed ( }>40 \%) \text { broadleaved forest or shrubland permanently flooded - Saline or brackish } \\
\text { water }\end{array}$ \\
\hline 18 & $\begin{array}{l}\text { Closed to open }(>15 \%) \text { grassland or woody vegetation on regularly flooded or waterlogged } \\
\text { soil }\end{array}$ \\
\hline 19 & Artificial surfaces and associated areas (Urban areas $>50 \%$ ) \\
\hline 20 & Bare areas \\
\hline 21 & Water bodies \\
\hline 22 & Permanent snow and ice \\
\hline 23 & Invalid pixels - No data (burnt areas, clouds) \\
\hline
\end{tabular}

Figure 5.1.: GlobCover Classification Map, ESA. 
interpolation has been performed on the GlobCover map.

Mountainous areas are typically affected by geometric distortion phenomena, such as shadow and layover, which generate misleading backscatter values, as explained in section 3.1.2. Areas affected by shadow appear extremely dark, while the ones affected by layover are very bright. Therefore, only pixels corresponding to low-relief terrain are taken into account, as in this case the impact of such errors is negligible. Given a DEM $D(x, y)$ of the considered area on ground, low- and high-relief terrain can be discriminated by setting a threshold on the predominant local slope, evaluated as presented in section 4.3.

An example of the impact of applying a threshold on the local slope for a single classification class is presented in Fig. 5.2, showing a nominal TanDEM-X SAR acquisition in $\mathrm{HH}$ polarization. It is characterized by the presence of both flat and mountainous terrain, and represents therefore a suitable test site for investigating the impact of topography on backscatter behavior. The slope map, derived from the corresponding DEM as in (4.7), is depicted in Fig. 5.2 (a).

Samples corresponding to the GlobCover class Mosaic cropland (50-70\%) /vegetation (grassland/shrubland/forest) (20-50\%) (class 3 in Fig. 5.1) are selected, as presented in Fig. 5.2 (b): red and blue areas correspond to such vegetation class over flat and mountainous terrain, respectively. The histograms of the selected $\beta^{0}$ samples are presented in Fig. 5.2 (c). Flat areas (red) are characterized by a mean $\beta^{0}$ of $-5.02 \mathrm{~dB}$. If mountainous areas only (blue) are discriminated as presented above and considered, $\beta^{0}$ is mainly affected by shadowing phenomena, leading to a lower mean $\beta^{0}$ of $-6.3 \mathrm{~dB}$. If both flat and mountainous areas are simultaneously considered, the obtained mean $\beta^{0}$ is equal to $-5.49 \mathrm{~dB}$, showing a difference of about $0.5 \mathrm{~dB}$ from the mean $\beta^{0}$ over flat regions only.

After the first selection based on the local slope, valid $\beta^{0}$ samples are then discriminated depending on the corresponding ground classification class to which they belong. Since at this point the position in space of a single pixel within the $\beta^{0}$ image is not relevant any more, the spatial coordinates $(x, y)$ are from now on to be omitted in the text for the sake of simplicity.

In order to better understand the dependency of radar backscatter properties on ground classification, an example is presented in Fig. 5.3. A single TanDEM-X mission acquisition, acquired in $\mathrm{HH}$ polarization over an agricultural area and characterized by the presence of several ground classification classes, has been used. The histograms of the radar brightness in $\mathrm{dB}$ for 6 different GlobCover classes are presented in Figure 5.3. The considered classification classes and the corresponding mean $\beta^{0}$ are:

- class 3 - Mosaic cropland (50-70\%) / vegetation (grassland/shrubland/forest) (20$50 \%)$, mean $\beta^{0}$ of $-5.02 \mathrm{~dB}$, 

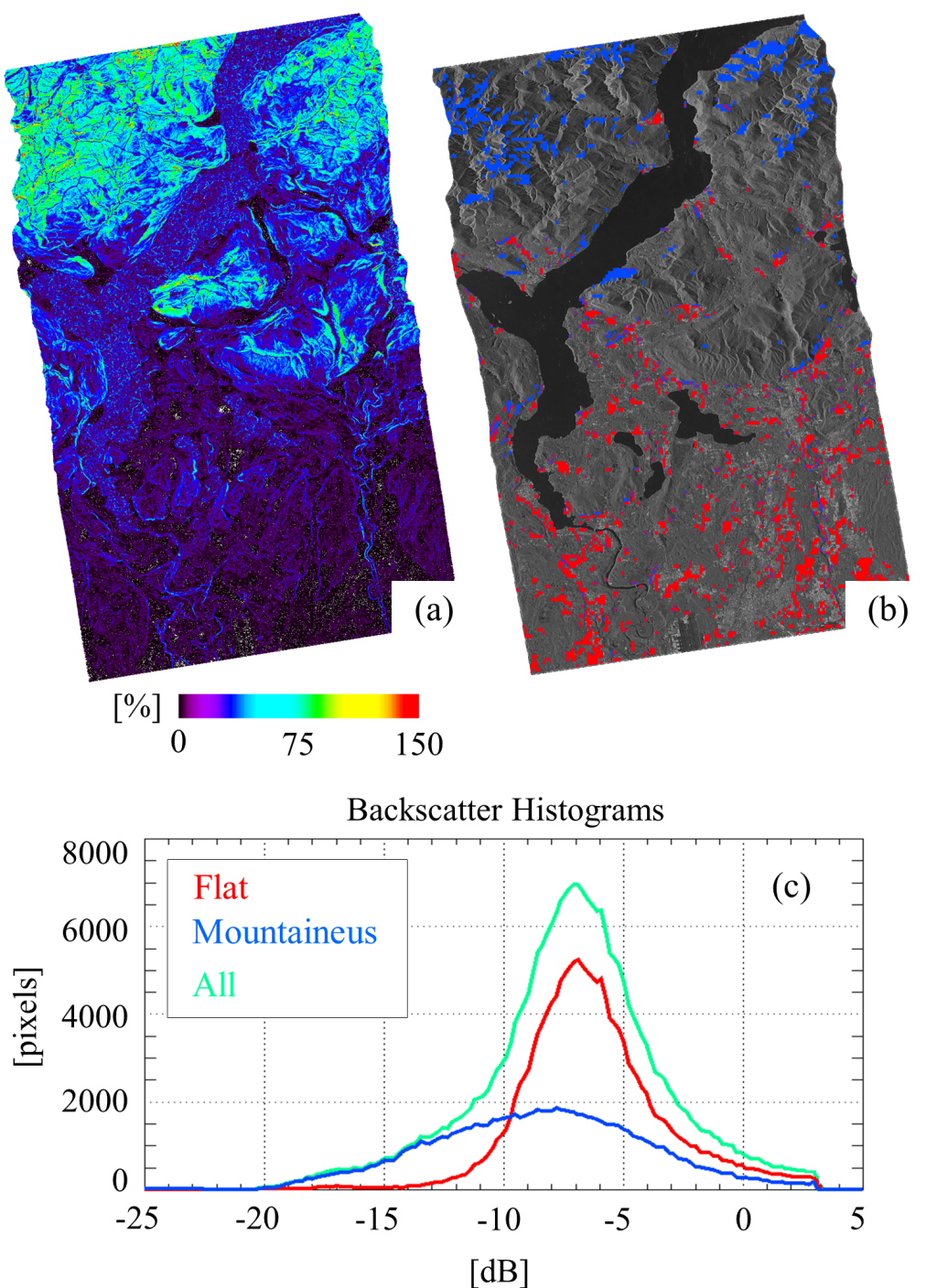

Figure 5.2.: Impact of applying a threshold on the local slope for the estimation of the mean $\beta^{0}$ from a TanDEM-X SAR image. (a) Slope map of the considered area, derived from the corresponding DEM as in (4.7). (b) Selected samples over flat (red) and mountainous (blue) areas for the GlobCover class Mosaic cropland (50-70\%) / vegetation (grassland/shrubland/forest) (20-50\%) (class 3 in Tab. 5.1). (c) Histograms of the selected $\beta^{0}$ samples: blue - samples belonging to mountainous areas only, red - samples belonging to flat areas only, green - combination of both samples belonging to mountainous and flat areas. 


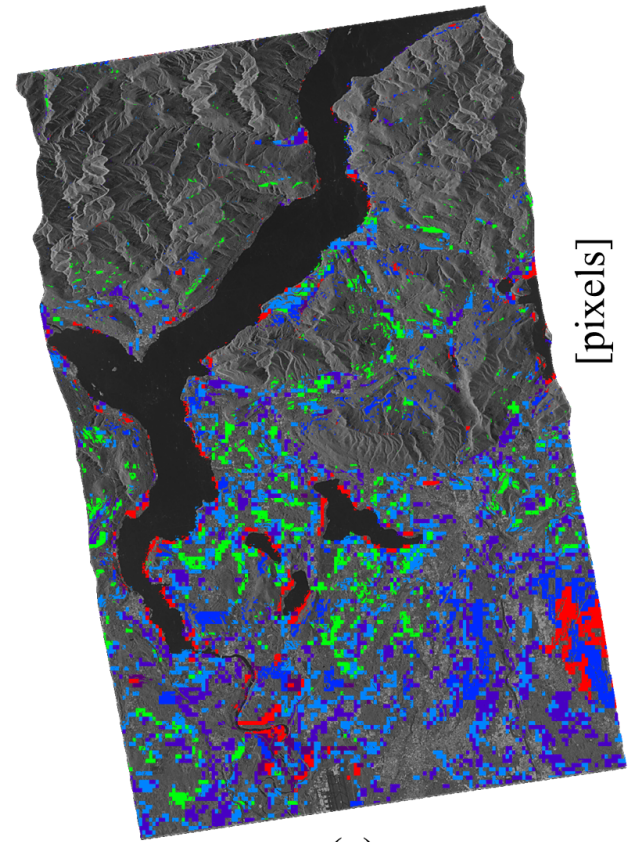

(a)

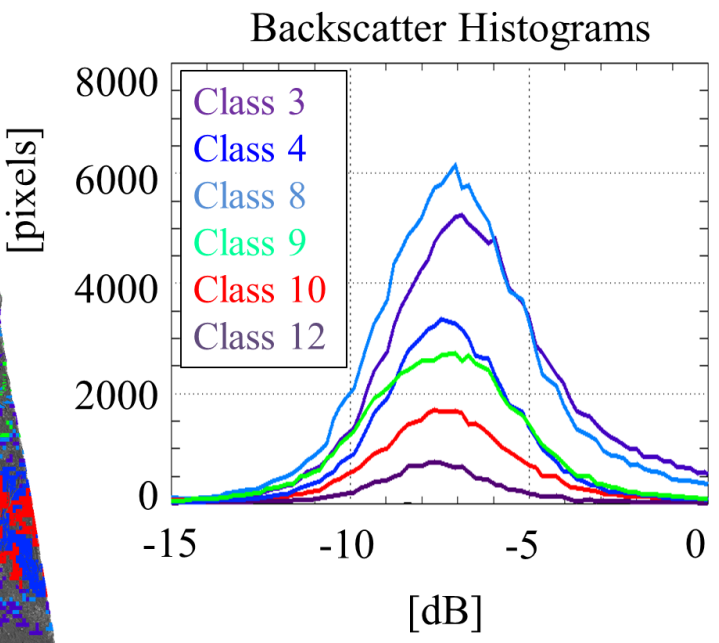

(b)

Figure 5.3.: Example of the dependency of the radar backscatter on ground classification. (a) Distribution of GlobCover classes, superimposed on a detected SAR image. (b) $\beta^{0}$ histograms of different ground classification classes from a single TanDEM-X acquisition in HH polarization. Only areas characterized by mean local slopes smaller than $20 \%$ are taken into account. The considered classification classes are: class 3 - Mosaic cropland (50-70\%) / vegetation (grassland/shrubland/forest) (20-50\%), class 4: Mosaic vegetation (grassland/shrubland/forest) (50-70\%) / cropland (20-50\%), class 8 Closed $(>40 \%)$ needleleaved evergreen forest $(>5 \mathrm{~m})$, class 9 - Open $(15-$ $40 \%)$ needleleaved deciduous or evergreen forest $(>5 \mathrm{~m})$, class 10 - Closed to open $(>15 \%)$ mixed broadleaved and needleleaved forest $(>5 \mathrm{~m})$, and class 12 - Mosaic grassland (50-70\%) / forest or shrubland (20-50\%)

- class 4: Mosaic vegetation (grassland/shrubland/forest) (50-70\%) / cropland (20$50 \%$ ), mean $\beta^{0}$ of $-5.74 \mathrm{~dB}$,

- class 8 - Closed ( $>40 \%)$ needleleaved evergreen forest $(>5 \mathrm{~m})$, mean $\beta^{0}$ of -6.54 $\mathrm{dB}$,

- class 9 - Open (15-40\%) needleleaved deciduous or evergreen forest ( $>5 \mathrm{~m})$, mean $\beta^{0}$ of $-6.92 \mathrm{~dB}$, 
- class 10 - Closed to open (>15\%) mixed broadleaved and needleleaved forest $(>5 \mathrm{~m})$, mean $\beta^{0}$ of $-6.4 \mathrm{~dB}$,

- class 12 - Mosaic grassland (50-70\%) / forest or shrubland (20-50\%), mean $\beta^{0}$ of $-6.78 \mathrm{~dB}$.

Besides the different number of available samples for each considered class, the $\beta^{0}$ distributions present different mean values and standard deviations as well, confirming the dependence of the radar backscatter properties on the ground classification.

A discussion on the introduction of errors due to geocoding, a threshold on the estimated local slope, and a wrong classification is provided in section 5.3.4.

A further sub-step consists in grouping samples together depending on the local incidence angle $\theta_{l}$, i.e. the angle between the radar wave incident direction and the normal direction to the scattering surface, as depicted in Fig. 2.5. In case of flat Earth, $\theta_{l}$ coincides with $\theta$. By taking into account pixels characterized by moderate local slopes only, one can assume from now on that the local incidence angle can be approximated by the incidence angle itself and, therefore,

$$
\theta_{l} \simeq \theta
$$

One can now consider an incidence angle spread, which goes from $\theta_{\min }$ to $\theta_{\max }$, and divide it into $N_{j}$ sub-intervals, small enough to consider the angular variation of $\beta^{0}$ within a single interval to be negligible.

For the $p-t h$ classification class available inside the $i-t h$ considered image, the radar brightness mean value $\beta_{j}^{0}(i, p)$, its variance $\sigma_{j}^{2}(i, p)$, and the total number of available samples $S_{j}(i, p)$ are evaluated, where the $j$ index identifies the incidence angle interval $\left(j=\left[1, \cdots, N_{j}\right]\right)$. Moreover, the normalized histogram of the initial $\beta^{0} \mathrm{~dB}$ distribution is evaluated as well, together with the mean squared error $r_{j}(i, p)$ between such a distribution and the Gaussian probability density function (pdf) which best fits the data [98]. The assumption of a Gaussian distribution of $\beta^{0} \mathrm{~dB}$ is driven by computational convenience, given the high volume of available input data to be analyzed. $r_{j}(i, p)$ is an indicator of how much the considered distribution is affected by the presence of outliers, which modify the shape of the histogram itself. The complete set of statistical parameters, derived from each single scene, together with additional information on the acquisition parameters represents a valuable input for the global characterization of radar backscatter for different ground classification types, incidence angles and polarizations. Fig. 5.4 presents the flowchart of the developed algorithm, which leads to the generation of a backscatter data base. In particular, all the following parameters are extracted and stored into a such a data base, which is the starting point for modeling backscatter behavior, as lately presented in section 5.3.2:

- $\beta_{j}^{0}(i, p)$ : mean radar brightness, 


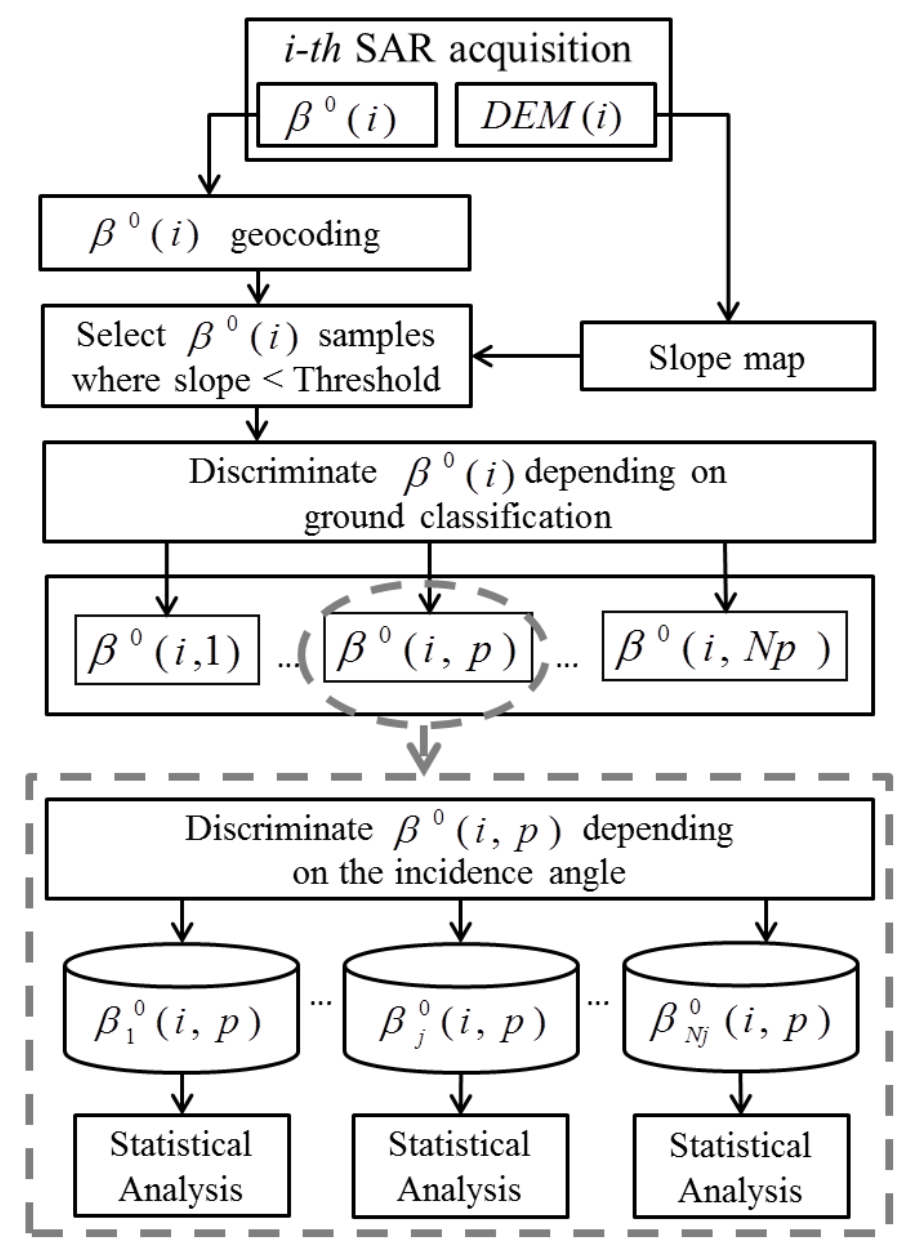

Figure 5.4.: Flowchart of the developed algorithm for the characterization of backscatter behavior from a single TanDEM-X scene. $\beta^{0}(i)$ and $D E M(i)$ represent respectively the radar brightness mean value of the $i-t h$ input QL image and the corresponding DEM. Index $p=\left[1, \cdots, N_{p}\right]$ identifies different ground classification classes, while $j=\left[0, \cdots, N_{j}\right]$ the incidence angle interval.

- $\sigma_{j}^{2}(i, p)$ : radar brightness variance,

- $S_{j}(i, p)$ : number of available samples,

- $r_{j}(i, p)$ : mean square error between the radar brightness distribution and a fitted Gaussian pdf,

- polarization channel,

- sensor center frequency, 
- acquisition date,

- orbit track,

- unique acquisition identifier.

\subsubsection{Modeling Algorithm}

Using the backscatter data base generated as described in section 5.3.1, a complete set of backscatter models can be derived to characterize the mean radar brightness. The idea is to model the backscatter behavior based on measurements from a high volume of input SAR images, which have been grouped together depending on polarization channel, local incidence angle, seasonal time and ground classification, improving the approach introduced in [98].

Two different models are generated, using the same estimation technique but first not including (unweighted modeling) and secondly including (weighted modeling) the quality of the input observations. A summary of the two methods output is finally given in section 5.3.3.

\section{Mean $\beta^{0}$ Unweighted Modeling}

For the $p-t h$ ground classification class, backscatter values, acquired from different images but within the same incidence angle interval, are first taken into account. Given $N_{j}$ incidence angle intervals, a vector $\boldsymbol{\beta}_{j}^{0}(p)$ of radar brightness mean values in $\mathrm{dB}$ is obtained for each $j-t h$ incidence angle interval:

$$
\boldsymbol{\beta}_{j}^{0}(p)=\left[\beta_{j}^{0}(1, p), \cdots, \beta_{j}^{0}\left(N_{n}, p\right)\right],
$$

where the $n-t h$ element $\beta_{j}^{0}(n, p)$ is the radar brightness mean value obtained by averaging all samples belonging to the $p-t h$ classification class within a single SAR image, and $N_{n}$ represents the available number of images for the $j-t h$ incidence angle interval. The $\boldsymbol{\beta}_{j}^{0}(p)$ vector is characterized by its mean value $\mu_{j}(p)$ and variance $\sigma_{j}^{2}(p)$. By repeating the operation for each $j-t h$ incidence angle interval, one can obtain a vector of $N_{j}$ radar brightness mean values:

$$
\boldsymbol{\mu}(p)=\left[\mu_{1}(p), \cdots, \mu_{N_{j}}(p)\right]
$$

and variances:

$$
\boldsymbol{\sigma}^{2}(p)=\left[\sigma_{1}^{2}(p), \cdots, \sigma_{N_{j}}^{2}(p)\right] .
$$

They can be used to generate an incidence angle dependent parametric backscatter model. If now the $p-t h$ vegetation class is considered, the system can be described as follows: 


$$
\boldsymbol{\mu}=\mathbf{Z}(\boldsymbol{\theta}, \boldsymbol{\xi})+\mathbf{w},
$$

where the dependency on the $p$ parameter has been omitted for the sake of simplicity. $\mathbf{Z}$ depends on the incidence angle vector $\boldsymbol{\theta}=\left[\theta_{1}, \cdots, \theta_{N_{j}}\right]$ and on the models's parameters vector $\boldsymbol{\xi}$ to be estimated, while $\mathbf{w}$ represents a noise contribution, modeled as a zero mean white Gaussian noise, which distribution is defined as:

$$
\mathbf{w} \sim \mathcal{N}(\mathbf{0}, \mathbf{C}),
$$

where $\mathbf{C}$ represents the covariance matrix. Assuming that different acquisitions are characterized by independent noise contributions, $\mathbf{C}$ can be described as a diagonal matrix, defined as:

$$
\mathbf{C}=\operatorname{diag}\left\{\boldsymbol{\sigma}^{2}\right\} \text {. }
$$

One can now assume to use an $m-t h$ order polynomial function to model the mean radar brightness behavior. In this case, the parametric model $\mathbf{Z}(\boldsymbol{\theta}, \boldsymbol{\xi})$ becomes linear with respect to the $\boldsymbol{\xi}$ parameters to be estimated, and can be written as:

$$
\boldsymbol{\mu}=\mathbf{H}_{\theta} \boldsymbol{\xi}+\mathbf{w}
$$

where

$$
\boldsymbol{\xi}=\left[\xi_{0}, \cdots, \xi_{m-1}\right]^{T}
$$

and

$$
\mathbf{H}_{\theta}=\left[\begin{array}{cccc}
1 & \theta_{1} & \cdots & \theta_{1}^{m-1} \\
\vdots & \vdots & \vdots & \vdots \\
1 & \theta_{N_{j}} & \cdots & \theta_{N_{j}}^{m-1}
\end{array}\right]
$$

The choice of a polynomial model resides in the fact that the target of the current approach is to develop a modeling technique which is as much as possible model-free, without needing a priori knowledge on the backscattering mechanism itself. The optimum estimator of the parameter vector is the maximum likelihood (ML) estimator, which can be derived by maximizing the probability density function of measured observations with respect to the parameters:

$$
\widehat{\boldsymbol{\xi}}=\arg \max _{\boldsymbol{\xi}} p[\boldsymbol{\mu} \mid \boldsymbol{\xi}]
$$

As presented in [99], $\widehat{\xi}$ can be obtained by minimizing the following quadratic form: 


$$
\widehat{\boldsymbol{\xi}}=\arg \min _{\boldsymbol{\xi}}\left\{\left[\boldsymbol{\mu}-\mathbf{H}_{\theta} \boldsymbol{\xi}\right]^{T} \mathbf{C}^{-1}\left[\boldsymbol{\mu}-\mathbf{H}_{\theta} \boldsymbol{\xi}\right]\right\}
$$

which leads to:

$$
\widehat{\boldsymbol{\xi}}=\left(\mathbf{H}_{\theta}^{T} \mathbf{C}^{-1} \mathbf{H}_{\theta}\right)^{-1} \mathbf{H}_{\theta}^{T} \mathbf{C}^{-1} \boldsymbol{\mu} .
$$

Since the model is linear with respect to the parameters to be estimated, the ML estimator corresponds to the minimum variance unbiased (MVU) estimator.

From experimental considerations it has been decided to use a third-order polynomial to model the mean radar brightness, defined as:

$$
\beta_{u n W}^{0}(\theta)=\widehat{\xi_{0}}+\widehat{\xi_{1}} \theta+\widehat{\xi_{2}} \theta^{2}+\widehat{\xi_{3}} \theta^{3} .
$$

where the nomenclature $\beta_{u n W}^{0}(\theta)$ underlines the fact that the estimation is based on unweighted $\beta^{0}$ mean values as input.

Note that all the derived models are considered to be valid only within the incidence angle spread where real observations are available.

\section{Mean $\boldsymbol{\beta}^{0}$ Weighted Modeling}

A further refinement in the estimation technique can be developed by taking into account the quality of the input measurements. This can be done by considering the number of available samples $S_{j}(n, p)$ and the mean square error $r_{j}(n, p)$, defined in section 5.3.1. A quality weight $a_{j}(n, p)$ can be associated to each input measurement $\beta_{j}^{0}(n, p)$, being defined as:

$$
a_{j}(n, p)=\sqrt{\frac{S_{j}(n, p)}{r_{j}(n, p)}} / \sum_{k=1}^{N_{n}} \sqrt{\frac{S_{j}(k, p)}{r_{j}(k, p)}} .
$$

The meaning of such weights is that measurements characterized by a lower number of available input observations or by a distribution that does not follow the shape of a theoretical one, and therefore most likely affected by outliers, are considered to be less reliable for the estimation process itself.

Starting from the $\boldsymbol{\beta}_{j}^{0}(p)$ vector as in (5.2), its weighted mean value can be evaluated by taking into account the quality weights in (5.15) as:

$$
\mu_{j}^{w}(p)=E\left[\boldsymbol{\beta}_{j}^{0}(p)\right]^{w}=\sum_{n=1}^{N_{n}} a_{j}(n, p) \beta_{j}^{0}(n, p) .
$$

The corresponding weighted variance $\sigma_{j}^{2 w}(p)$ is then given by: 


$$
\sigma_{j}^{2 w}(p)=\sum_{n=1}^{N_{n}} a_{j}^{2}(n, p) \sigma_{j}^{2}(n, p) .
$$

By grouping together all the available $N_{j}$ incidence angle intervals, one obtains a vector of $N_{j}$ radar brightness weighted mean values:

$$
\boldsymbol{\mu}^{w}(p)=\left[\mu_{1}^{w}(p), \cdots, \mu_{N_{j}}^{w}(p)\right]
$$

and weighted variances:

$$
\boldsymbol{\sigma}^{2 w}(p)=\left[\sigma_{1}^{2 w}(p), \cdots, \sigma_{N_{j}}^{2 w}(p)\right] .
$$

Again, the system can be modeled as presented in (5.8), this time by substituting the $\beta^{0}$ mean values vector $\boldsymbol{\mu}$ by its weighted version introduced in (5.18) and by defining a new noise contribution $\mathbf{w}_{w}$ as:

$$
\mathbf{w}_{w} \sim \mathcal{N}\left(\mathbf{0}, \mathbf{C}_{w}\right),
$$

where the covariance matrix $\mathbf{C}_{w}$ is defined as:

$$
\mathbf{C}_{w}=\operatorname{diag}\left\{\boldsymbol{\sigma}^{2 w}\right\} .
$$

The linear model in (5.8) can therefore be modified as:

$$
\boldsymbol{\mu}^{w}=\mathbf{H}_{\theta} \boldsymbol{\xi}^{w}+\mathbf{w}_{w}
$$

where:

$$
\boldsymbol{\xi}^{w}=\left[\xi_{0}^{w}, \cdots, \xi_{m-1}^{w}\right]^{T}
$$

which leads to the estimation of a vector $\widehat{\xi^{w}}$ of fitting coefficients as:

$$
\widehat{\boldsymbol{\xi}^{w}}=\left(\mathbf{H}_{\theta}^{T}\left(\mathbf{C}_{w}\right)^{-1} \mathbf{H}_{\theta}\right)^{-1} \mathbf{H}_{\theta}^{T}\left(\mathbf{C}_{w}\right)^{-1} \boldsymbol{\mu}^{w}
$$

The mean radar brightness $\beta_{W}^{0}(\theta)$, can finally be modeled as:

$$
\beta_{W}^{0}(\theta)=\widehat{\xi_{0}^{w}}+\widehat{\xi_{1}^{w}} \theta+\widehat{\xi_{2}^{w}} \theta^{2}+\widehat{\xi_{3}^{w}} \theta^{3}
$$

where this time the nomenclature $\beta_{W}^{0}(\theta)$ underlines the fact that the estimation is based on weighted $\beta^{0}$ mean values. 


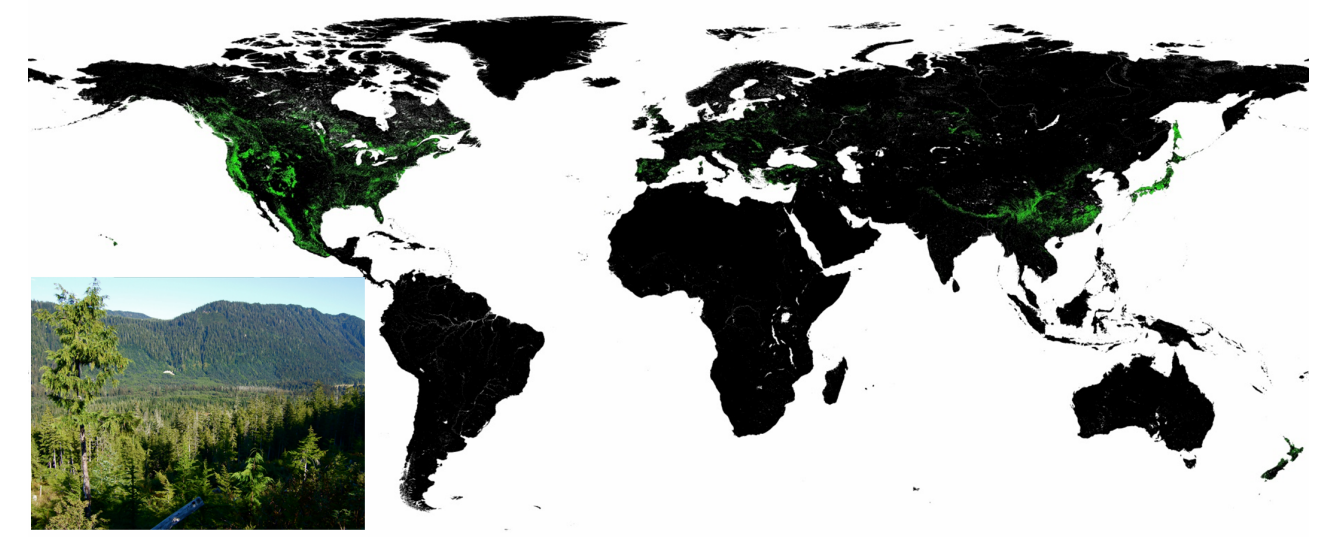

Figure 5.5.: Location and characteristics of the Closed (>40\%) needleleaved evergreen forest ( $>5 \mathrm{~m}$ ) GlobCover class shown in green (class number 8 in Fig. 5.1).

\subsubsection{Summary of methods output}

As an example, both backscatter modeling techniques, presented in section 5.3.2 (unweighted/weighted), are used to model the Closed (>40\%) needleleaved evergreen forest (>5m) GlobCover class (class 8 in Fig. 5.1), which corresponds to a typical boreal forest. Its location on the planisphere is shown in Fig. 5.5. It is important to note that about 90,000 scenes of $30 \mathrm{~km} \times 50 \mathrm{~km}$ located all around the world, where such a class is detected, are simultaneously taken into account, confirming the idea of a global approach for modeling backscatter behavior.

Figure 5.6 (a) presents the results obtained by applying the described backscatter modeling algorithm: violet rhomboidal marks represent the radar brightness mean values $\boldsymbol{\mu}(p)$, for each incidence angle interval, evaluated by simply averaging the $\boldsymbol{\beta}_{j}^{0}(n, p)$ mean values from each available input image:

$$
\boldsymbol{\mu}(p)=\left[\mu_{1}(p), \cdots, \mu_{N_{j}}(p)\right],
$$

where index $p$ identifies the GlobCover class number in Fig. 5.1 (in this case $p=8$ ). The corresponding confidence ranges of the measurements are displayed as well, and labeled as $\boldsymbol{\mu}(p)+\boldsymbol{\sigma}(p)$ and $\boldsymbol{\mu}(p)-\boldsymbol{\sigma}(p)$, where:

$$
\boldsymbol{\sigma}(p)=\left[\sqrt{\sigma_{1}^{2}(p)}, \cdots, \sqrt{\sigma_{N_{j}}^{2}(p)}\right]
$$

The red squared marks identify the $\beta^{0}$ weighted mean values $\boldsymbol{\mu}^{w}(p)$, obtained by applying the weighting procedure explained in (5.15) and (5.16). The continuous lines identify the corresponding fitted models, derived from the original $\beta^{0}$ mean values $\left(\beta_{u n W}^{0}(\theta)\right.$ in green) and from the weighted ones $\left(\beta_{W}^{0}(\theta)\right.$ in blue), respectively. Finally, Fig. 5.6 (b) 

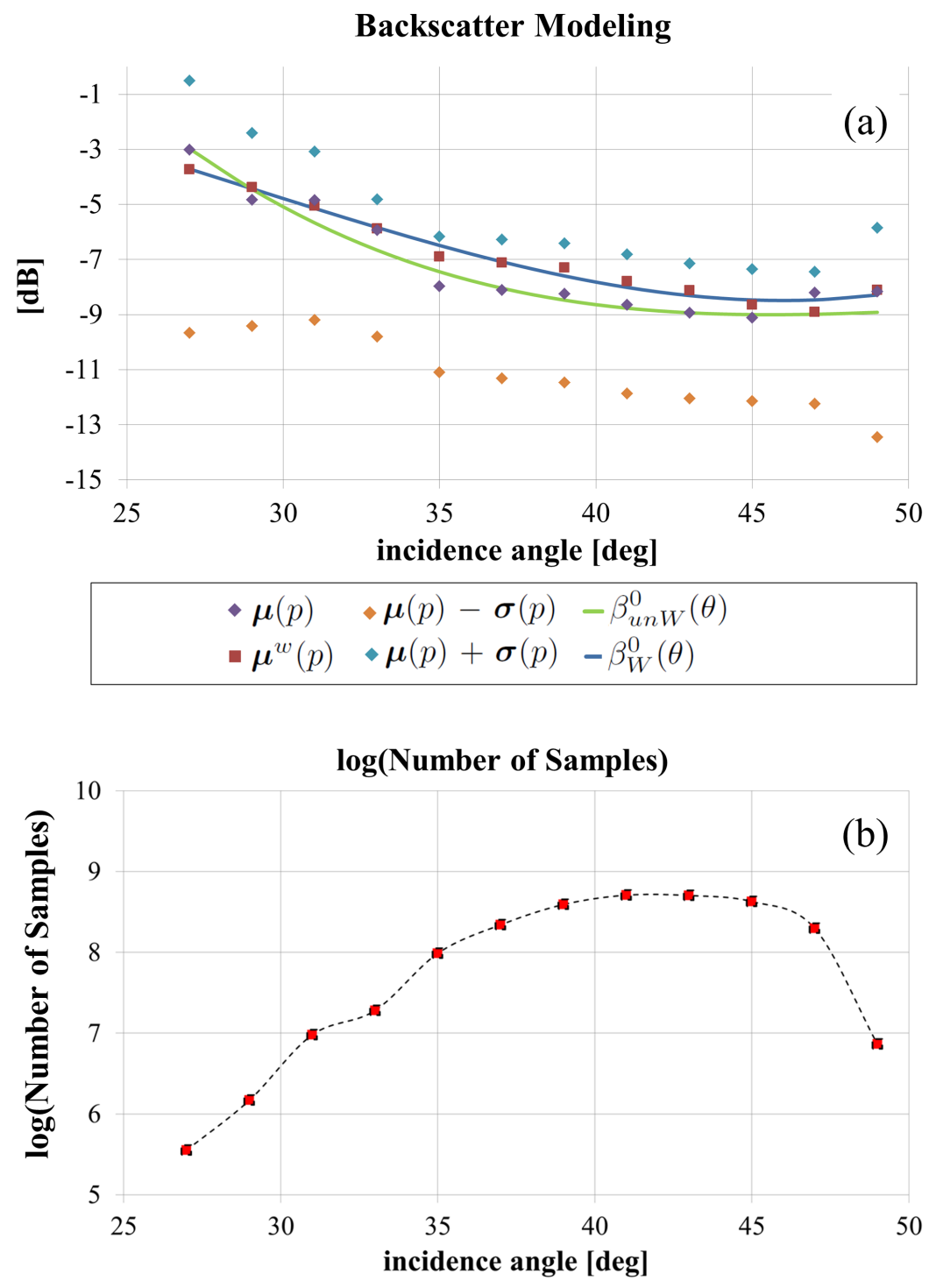

Figure 5.6.: (a) Estimated mean $\beta^{0}$ models: $\boldsymbol{\mu}(p)$ is the vector of radar brightness mean values, plotted together with its confidence ranges $\boldsymbol{\mu}(p)+\boldsymbol{\sigma}(p)$ and $\boldsymbol{\mu}(p)-\boldsymbol{\sigma}(p), \boldsymbol{\mu}^{w}(p)$ identifies the $\beta^{0}$ weighted mean values, while $\beta_{u n W}^{0}(\theta)$ and $\beta_{W}^{0}(\theta)$ are the corresponding fitted mean $\beta^{0}$ models, derived from the original $\beta^{0}$ mean values and from the weighted ones, respectively. (b) Total number of considered samples for each incidence angle interval (in logarithmic scale). 
shows the total number of available input samples in logarithmic scale for each incidence angle interval. As it can be seen, the series of $\boldsymbol{\mu}^{w}(p)$ presents less discontinuities between neighbor measurements with respect to $\boldsymbol{\mu}(p)$. The root mean square errors (computed in $\mathrm{dB}$ ) between the $\beta^{0}$ mean values and the corresponding fitted model are evaluated as:

$$
\begin{aligned}
& R M S E_{u n W}=\sqrt{E\left[\left(\boldsymbol{\mu}(p)-\beta_{u n W}^{0}(\theta)\right)^{2}\right]} \\
& R M S E_{W}=\sqrt{E\left[\left(\boldsymbol{\mu}^{w}(p)-\beta_{W}^{0}(\theta)\right)^{2}\right]}
\end{aligned}
$$

from which one can obtain $R M S E_{u n W}=0.46 \mathrm{~dB}$, and $R M S E_{W}=0.23 \mathrm{~dB}$, which shows that the weighting process, which takes into account both the quality and the quantity of the input measurements, helps for equalizing the input observations to values which can provide a better fitting.

By properly grouping together images acquired with different acquisition parameters such as sensor center frequency, polarization, and incidence angle, the algorithm can be separately applied to all the available ground vegetation classes, leading to the generation of a complete archive of $\beta^{0}$ models for X-band backscatter.

From an analysis on dedicated test sites, no significant difference between ascending and descending orbit tracks has been detected, as explained in section 5.4.3. Therefore, both acquisition geometries have been taken into account for the current analysis. Nevertheless, this last aspect has been separately addressed for the analysis of the backscatter from tropical rainforests, presented in section 5.6.

Moreover, radar backscatter is strongly affected by the seasonal conditions in which an acquisition is performed; this aspect is better detailed in section 5.5.

Finally, as mentioned in section 2.2.3, the backscattering coefficients $\sigma^{0}$ and $\gamma^{0}$ can be directly derived from the radar brightness $\beta^{0}$. The $\beta_{W}^{0}(\theta)$ model is plotted in Fig. 5.7, together with the corresponding $\sigma_{W}^{0}(\theta)$ and $\gamma_{W}^{0}(\theta)$, derived from $\beta_{W}^{0}(\theta)$ by compensating for the sine and the tangent of the incidence angle $\theta$, respectively. Such quantities typically present a smaller angular variation, and are commonly used within the scientific community for a large number of applications, i.e. soil moisture estimation from $\sigma^{0}$ [100], and radar antenna pattern verification from $\gamma^{0}$ [101].

\subsubsection{Discussion upon the Sources of Error}

Different sources of errors can affect the accuracy of the derived backscatter models, in particular:

- Geocoding errors: the geolocation of the absolutely calibrated amplitude quicklooks is obtained by performing a bilinear interpolation on a coarse geocoding 


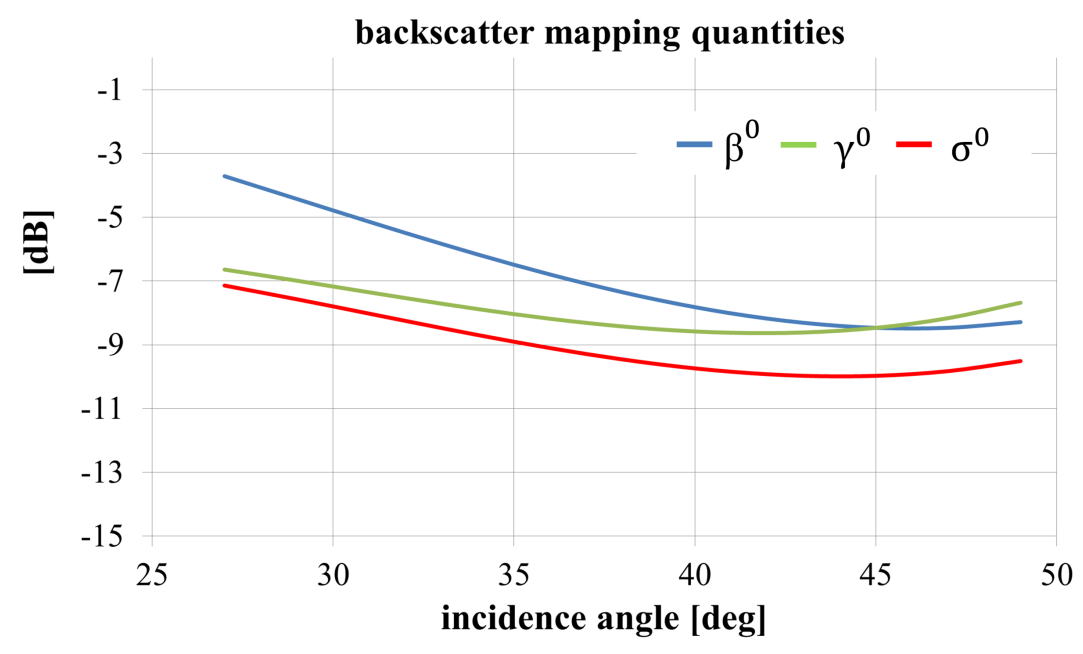

Figure 5.7.: Estimated backscatter characteristics for the Closed (>40\%) needleleaved evergreen forest (>5m) GlobCover class (number 4 in Fig. 5.1), in $\mathrm{HH}$ polarization. $\beta_{W}^{0}(\theta)$ is the mean radar brightness model derived as in (5.25), $\sigma_{W}^{0}(\theta)$ and $\gamma_{W}^{0}(\theta)$ are the backscattering coefficients derived from $\beta_{W}^{0}(\theta)$.

grid, provided within the TanDEM-X quicklook product itself, as explained in section 4.3. The grid projects a SAR coordinate on the DEM surface. Several DEM sources have been used for the operational production of geocoded products, as mentioned in [80]. The elevation accuracy of the considered DEM might locally vary, affecting the pixel location accuracy itself. Taking into account the SRTM DEM specification of $16 \mathrm{~m}$ of vertical accuracy [23], for nominal TanDEM-X products one can expect a pixel geolocation error up to $20 \mathrm{~m}$ [80]. Such an error corresponds to less than a pixel within the input $\beta^{0}$ quicklook images (with resolution of $50 \mathrm{~m} \times 50 \mathrm{~m}$ ). Moreover, since high-relief terrain is not being taken into account, location errors due to the bilinear interpolation of the mapping grid can be considered negligible.

- Local incidence angle estimation errors: as for the geolocation, a rough grid of incidence angles is provided within the TanDEM-X quicklook product and interpolated for each required pixel. The choice of erasing pixels characterized by high local slopes aims at reducing the impact of a misleading approximation of the local incidence angle with the incidence angle itself, as explained in section 5.3.1.

- Atmospheric conditions: radar backscatter can be affected by the presence of rain, wet soil, and general atmospheric perturbations, leading to backscatter variations which can be in the order of dBs [102]. However, the annual probability of strong meteorological events is relatively low [102] and the use of a high number of input data to perform the statistical analysis, as well as the introduction of quality 
weights, should significantly mitigate the effect of such outliers.

- Classification errors: several aspects can affect the quality of the classification of the ground using the GlobCover map. First of all, the GlobCover resolution itself is $300 \mathrm{~m} \times 300 \mathrm{~m}$, which is lower than the one of the considered input quicklook data, and can therefore lead to classification errors. Secondly, errors can occur due to modifications in time of the illuminated area or to a wrong classification of the ground itself (an example over the Amazon Rain Forest is given in section 5.4.1). All these kinds of errors are seen as outliers within the estimation algorithm. Their effect is mitigated by the high amount of TanDEM-X input data and by the use of quality weights as presented in section 5.3.2. Other classification maps could also be used to validate and improve the derived models. A significant scenario for classification errors of the ground is represented by Greenland ice sheet. From [103], it is known that ice sheet can be classified into different types, depending on snow characteristics. Nevertheless, the GlobCover classification map provides only one single class for Greenland: Permanent snow and ice. Therefore a dedicated analysis has to be performed, in order to refine snow classification into different sub-classes, which can be correctly associated to different kinds of snow. This topic is the core of chapter 6 .

- Geometric distortion errors: as previously explained in section 5.3.1, areas affected by geometric distortion phenomena, such as shadow and layover, present a backscatter behavior which diverge from the theoretical one. Moreover, the extension of such regions depends on the acquisition geometry, for example if an area acquired with a near-range beam (low incidence angles) is not affected by shadow, it could instead end up in a shadow region if acquired using a far-range beam (high incidence angle). In order to mitigate such effects, only pixels corresponding to low-relief terrain are taken into account. Nevertheless, flat areas located in the neighborhood of mountainous ones can still be affected by geometric distortion. In order to quantify the error introduced by using the slope information only, an example over a dedicated test site is presented. A worst case scenario is shown in Fig. 5.8, where an acquisition performed over high-relief terrain is used for the current analysis. The quicklook amplitude is displayed in Fig. 5.8 (a), geocoded using the coarse mapping grid as presented in section 5.3.1. Geometric distortion effects are clearly visible. One can now compare results obtained from the slope map and from a more accurate way for the estimation of shadow and layover. Given the insufficient reliability of the SRTM DEM [104] over high-relief terrain, a way to do so is to take into account both amplitude and interferometric coherence, and use a series of thresholds in order to discriminate shadow (characterized by low values 

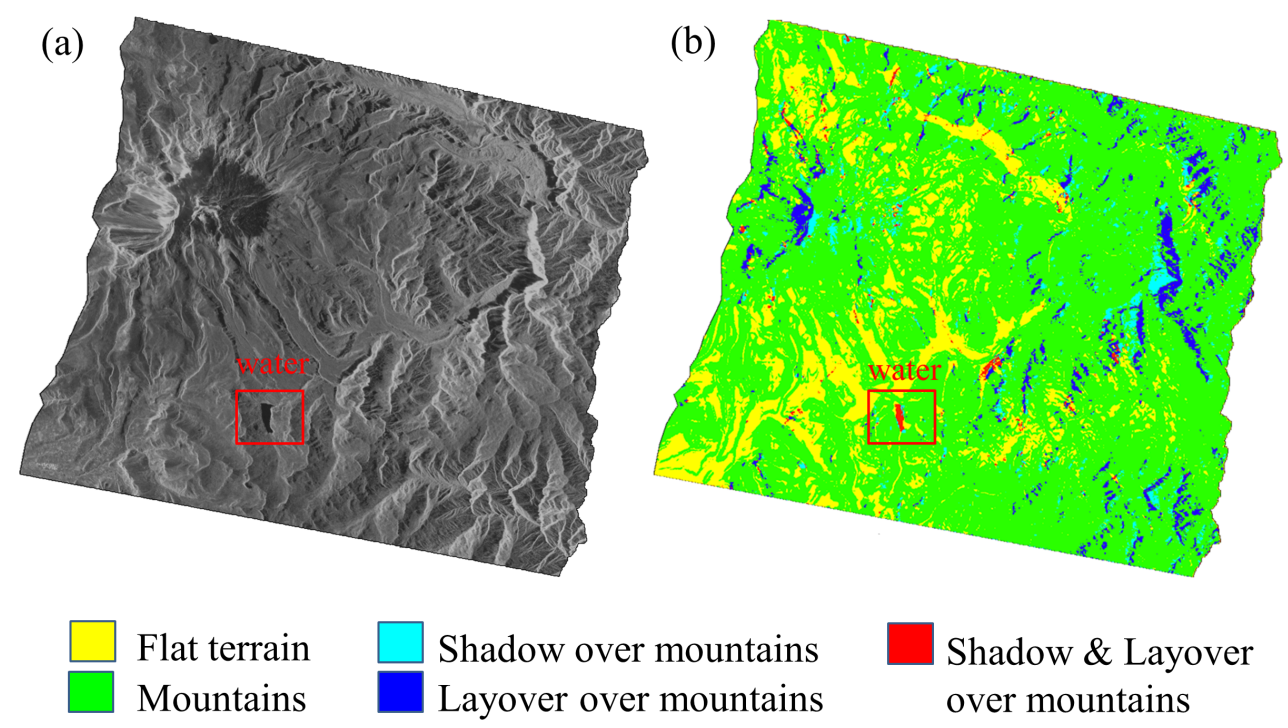

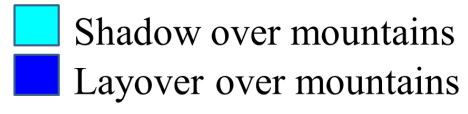

Shadow \& Layover over mountains

Figure 5.8.: Example of quantification of geometric distortion errors. (a) Geocoded amplitude image of a TanDEM-X acquisition over the Andes. (b) Color coded map showing: yellow and green - flat and mountainous terrain, discriminated from the associated slope map, light blue and blue - shadow and layover areas, discriminated using amplitude and interferometric coherence, red Shadow or layover areas mapped as flat terrain.

of both amplitude and interferometric coherence ${ }^{1}$ ), and layover (characterized by high amplitude and low interferometric coherence) [105]. The interferometric coherence layer, available within each TanDEM-X quicklook product, has been used for this purpose. Due to the high computational effort of shadow/layover detection, only a worst case scenario is analyzed. Fig. 5.8 (b) presents a color coded map showing the following information:

- yellow and green: flat and mountainous terrain, discriminated from the associated slope map by setting a threshold at $20 \%$. Samples corresponding to flat areas are considered to be reliable for the current backscatter analysis,

- light blue and blue: shadow and layover areas, discriminated using amplitude and interferometric coherence,

- red: Shadow or layover areas which are mapped as flat terrain, and represent therefore a source of error for the current backscatter analysis.

For the considered example, characterized by extremely high-relief terrain, the

${ }^{1}$ Water presents the same characteristics, and therefore cannot be separated from shadow by such a technique. Water pixels are identified by the GlobCover map. 
overall percentage of shadow or layover areas, which are mapped as flat terrain is below $4 \%$. Given the high number of available input data, acquired over low-relief terrain, such percentage does not represent a critical source of error.

\subsection{Performance Evaluation Approach of Global Backscatter Models}

In order to verify the accuracy of the backscatter models, generated as in sections 5.3.1 and 5.3.2, two aspects have to be taken into account:

1. Check for remaining absolute offsets,

2. Verification of the model's relative curvature and slope accuracy.

The implemented verification procedures are explained in sections 5.4.1 and 5.4.2, respectively. Both aspects are related to the presence of the sources of errors presented in section 5.3.4. By repeating the intended approach over different test sites and grouping the results together, it is possible to get an overview of the overall accuracy of the derived backscatter models, as it is presented in section 5.4.3.

\subsubsection{Check for Absolute Offsets}

In order to verify the presence of residual offsets in the derived models, a single $\beta^{0}$ geocoded image is considered. The intensity of such an image can be simulated by feeding the modeled backscatter into the original acquisition geometry and classification of this scene. For each input $\beta^{0}(m, n)$ pixel, where the $m$ and $n$ indices identify the horizontal and vertical dimensions (in longitude and latitude coordinates), respectively, the associated incidence angle $\theta(m, n)$ and land cover classification $p(m, n)$ can be retrieved from the acquisition parameters and from the classification map. Depending on these two parameters and on the characterizing polarization, the corresponding radar brightness model can be evaluated and associated to the pixel value $\beta_{\text {sim }}^{0}(m, n)$ within the simulated image. For example, using the set of mean radar brightness weighted models $\beta_{W}^{0}$, one has:

$$
\beta_{\text {sim }}^{0}(m, n)=\beta_{W}^{0}(p(m, n), \theta(m, n)),
$$

where the polarization dependency has been omitted for the sake of simplicity. By evaluating the difference between the original input image and the simulated one, remaining offsets can be detected and quantified.

An example is presented in Fig. 5.9, where a TanDEM-X acquisition over the Amazon rain forest, acquired in $\mathrm{HH}$ polarization and with incidence angles between $46.8^{\circ}$ 


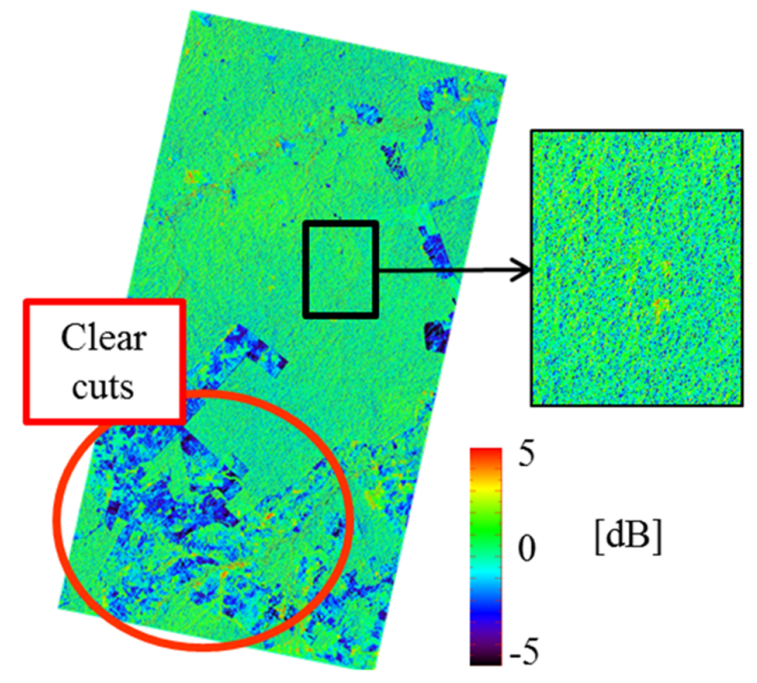

(a)

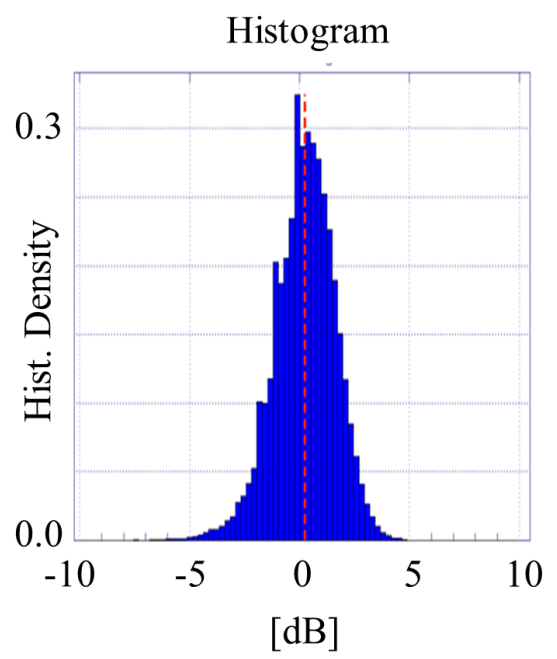

(b)

Figure 5.9.: Amazon rain forest test site. (a) Difference between the input $\beta^{0}$ image and a simulated one, using the TanDEM-X weighted backscatter models. The predominant ground classification class is Closed to open $(>15 \%)$ mixed broadleaved and needleleaved forest $(>5 \mathrm{~m})$ (class 10 in Fig. 5.1). Clear cuts are clearly visible (blue areas). (b) Histogram of the difference between the input $\beta^{0}$ image and the simulated one. It is characterized by a mean value of $0.2 \mathrm{~dB}$ and a standard deviation of $1.3 \mathrm{~dB}$.

and $48.5^{\circ}$, is considered. The predominant ground classification class from GlobCover is Closed to open (>15\%) mixed broadleaved and needleleaved forest ( $>5 \mathrm{~m}$ ) (class 10 in Fig. 5.1). Fig. 5.9 (a) shows the difference between the original input image and the simulated one using the weighted mean $\beta^{0}$ models. Interestingly, one can see that clear cuts are clearly visible within the red circle. This is due to a wrong classification of the ground. The reason for this is that this image was acquired two years after the GlobCover map was delivered. At the time of its delivery in 2009, deforestation had not affected this region yet, while it is clearly visible within the TanDEM-X acquisition. As already mentioned in section 5.3.4, such classification errors are considered as outliers within the backscatter models estimation process. Nevertheless, they also represent a potential starting point for change detection over larger time spans, by comparing actual TanDEM-X or TerraSAR-X data with simulated SAR images derived from the 2009 GLOBCOVER data and the derived backscatter models. For a realistic check of absolute offsets within our modeling results, a stable and homogenous region inside the test site has been selected. Fig. 5.9 (b) shows the histogram of the difference matrix for the con- 


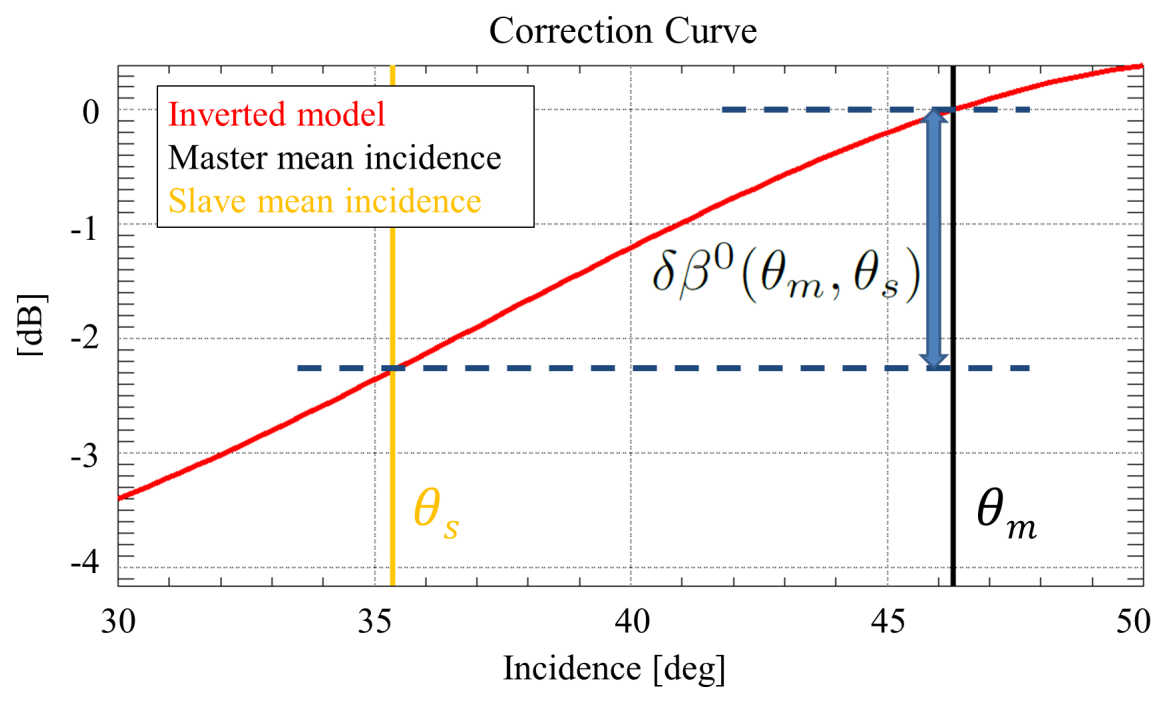

Figure 5.10.: Basic concept for the generation of a backscatter correction curve. $\delta \beta^{0}\left(\theta_{m}, \theta_{s}\right)$ represents the correction factor which has to be applied to a sample belonging to the slave image, with incidence angle $\theta_{s}$, in order to refer it to the same incidence angle $\theta_{m}$ of the correspondent pixel within the master image.

sidered region displayed within the small square in Fig. 5.9 (a). The histogram's mean value is about $0.2 \mathrm{~dB}$, with a standard deviation of $1.3 \mathrm{~dB}$.

\subsubsection{Curvature and Slope Accuracy}

To verify the model's relative curvature and slope accuracy, two SAR images, characterized by an overlapping ground area and acquired with the same polarization but with different incidence angles, are to be considered: one out of the two is taken as reference and therefore called master; while the other one is called slave. After being interpolated over the same ground coordinates, image samples from each single input image are grouped together depending on the corresponding ground classification class. For each available class, a separate analysis is performed. The corresponding mean $\beta^{0}$ model is considered and it is evaluated at the master and slave local incidence angles. By inverting such models as presented in [98], one can evaluate a correction factor $\delta \beta^{0}\left(\theta_{m}, \theta_{s}\right)$ per pixel to be applied in order to transform the slave image $\beta^{0}$ pixel (at incidence angle $\theta_{s}$ ) into the corresponding $\beta^{0}$ value that it would assume if acquired at the master image incidence angle $\theta_{m}$. The basic concept is explained in Fig. 5.10, where a correction fac- 


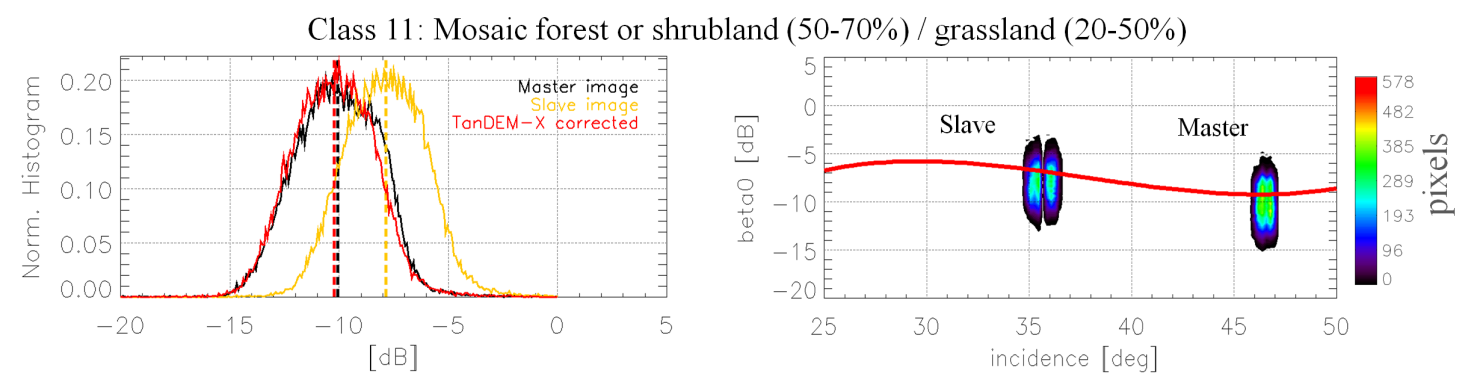

Class 12: Mosaic grassland (50-70\%) / forest or shrubland (20-50\%)
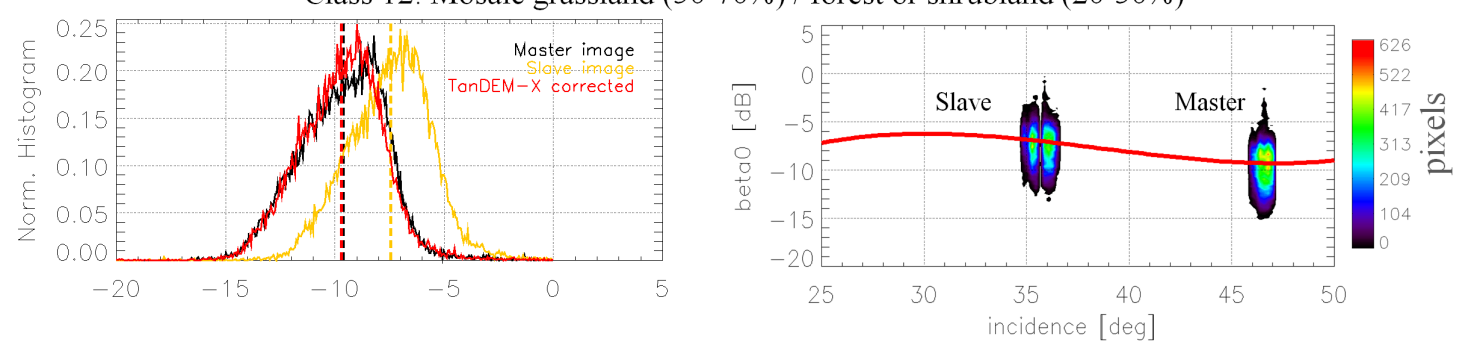

Class 14: Closed to open ( $>15 \%$ ) herbaceous vegetation (grassland, savannas or lichens/mosses)
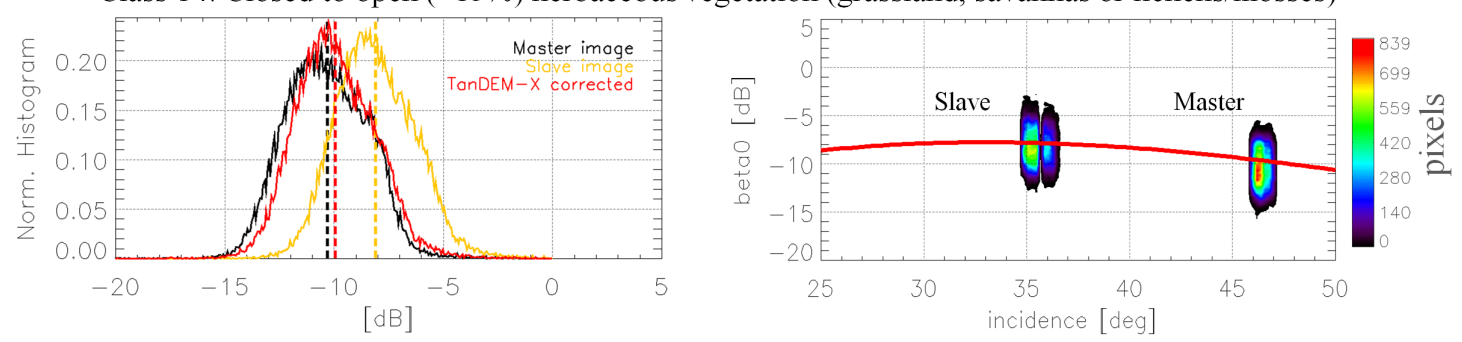

Figure 5.11.: Example of backscatter model accuracy verification approach over an agricultural area in England. Class 11, 12, and 14 in Fig. 5.1 are considered. Left-hand side: $\beta^{0}$ histogram of the master (black), the slave (orange) and the slave corrected to the master incidence angles (red), by applying the correction factors obtained as presented in Fig. 5.10. Right-hand side: $\beta^{0}$ models overplotted to the bi-dimensional histograms of the $\beta^{0}$ distributions for both the master and the slave images. The displayed colorbars identify the number of input $\beta^{0}$ pixels composing such histograms.

tor is derived. From the accuracy of the derived correction curves it is possible to define the accuracy of the models' curvature as well.

Fig. 5.11 presents an example of such an approach by using two images acquired over agricultural areas in England with $\mathrm{HH}$ polarization during March (master image) and September 2011 (slave image), respectively, and characterized by an overlapping region. The master image has a mean incidence angle of about $45^{\circ}$, while the slave one is cen- 
tered around $34^{\circ}$. Three different ground classification classes are taken into account: class 11, 12, and 14 in Fig. 5.1. The plots on the left-hand side show the $\beta^{0} \mathrm{~dB}$ histograms of the master (black), the slave (orange) and the slave corrected to the master incidence angles (red), by applying the correction factors obtained as presented in Fig. 5.10 .

All the histograms are evaluated by taking into account the overlapping region only and the plotted vertical dotted lines identify the histograms' mean values. The mean difference between the distributions of the master image and of the corrected slave varies from $0.16 \mathrm{~dB}$ to $0.7 \mathrm{~dB}$ for the three considered GlobCover classes. A good accordance can be found between the master images histograms and the slave corrected ones. Moreover, in order to visually analyze the presence of remaining absolute offsets for each class, separately, on the right-hand side of Fig. 5.11, the $\beta^{0}$ models are overplotted to the bi-dimensional histograms of the $\beta^{0}$ distribution for both the master and the slave images.

\subsubsection{Combination of Different Test Sites}

The verification of a backscatter model using the presented approach can be considered reliable only if repeated over several test sites, allowing for the analysis of an higher number of measurements.

In this section, a concrete example for the verification of a single class, taking into account data coming from different test sites, is presented. The considered class is closed to open $(>15 \%)$ broadleaved evergreen or semi-deciduous forest $(>5 \mathrm{~m}$ ) (class 5 in Fig. 5.1). Its global distribution is displayed in Fig. 5.12 (a). Ten different test sites have been taken into account, eight of which acquired over the Amazon rain forest and the other two over the African rain forest, shown in Fig. 5.12 (b) and (c), respectively. Each test site is composed by two overlapping acquisitions in HH polarization. A summary of the complete data set is presented in Tab. 5.1. Different incidence angles, as well as both ascending and descending orbits are taken into account.

Both $\beta_{u n W}^{0}(\theta)$ and $\beta_{W}^{0}(\theta)$ models, derived as presented in (5.14) (starting from unweighted $\beta^{0}$ mean values) and (5.25) (from weighted $\beta^{0}$ mean values), respectively, are considered, allowing for a comparison of the obtained performance.

Check for absolute offsets. The remaining offsets between real SAR data and the developed models are presented in Fig. 5.13: for each test site in Tab. 5.1, the master image mean radar brightness $E\left[\boldsymbol{\beta}_{m}^{0}\right]$ (red rhombus), the slave image mean radar brightness $E\left[\boldsymbol{\beta}_{s}^{0}\right]$ (yellow rhombus), and the corresponding $\beta_{u n W}^{0}\left(E\left[\boldsymbol{\theta}_{m}\right]\right)$ (green) and 


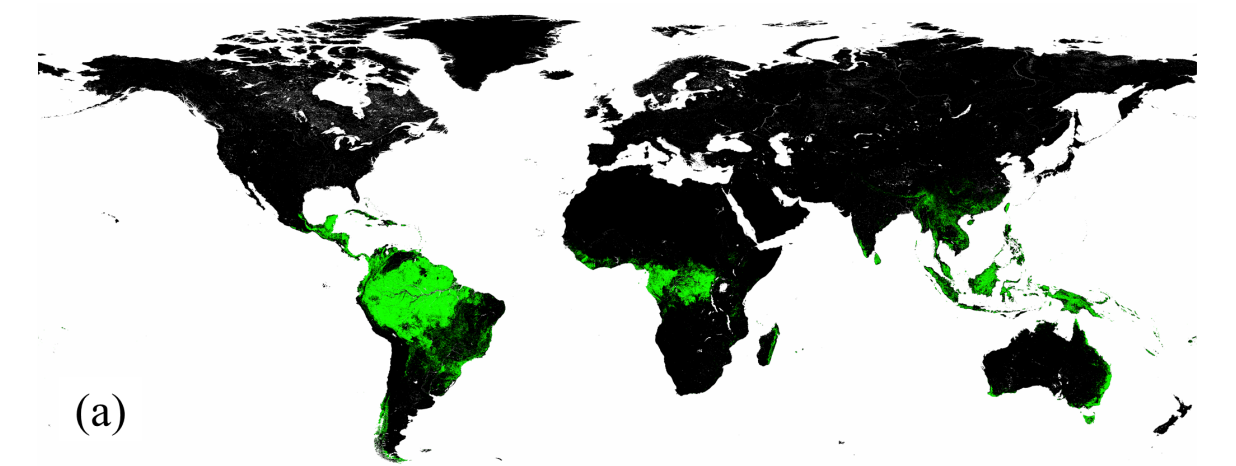

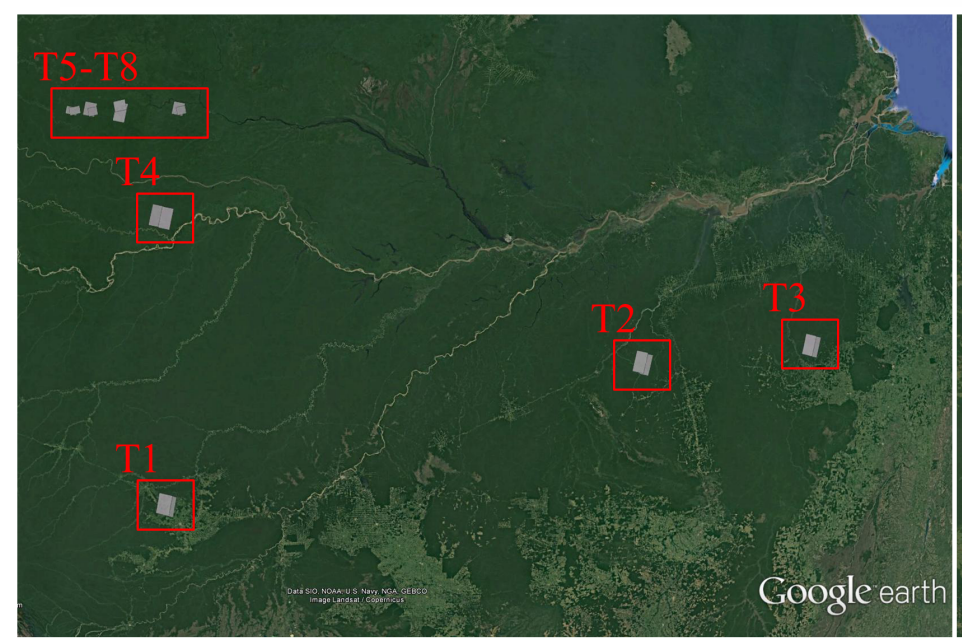

(b)

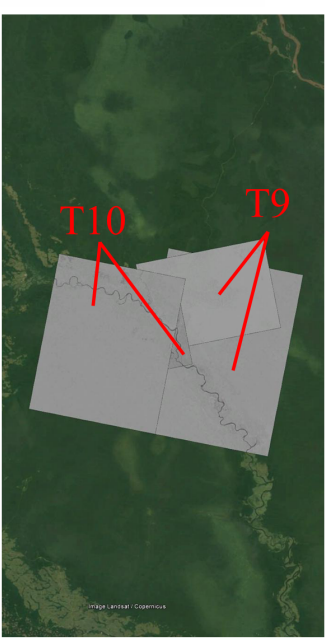

(c)

Figure 5.12.: (a) Global distribution of the closed to open (>15\%) broadleaved evergreen or semi-deciduous forest ( $>5 \mathrm{~m}$ ) GlobCover class (class 5 in Fig. 5.1). Test sites location over the Amazon rain forest (b) and the Congo rain forest (c) used for the backscatter model's verification.

$\beta_{W}^{0}\left(E\left[\boldsymbol{\theta}_{m}\right]\right)$ (light blue) models are depicted. The horizontal axis shows the master and slave images mean incidence angles. One can now evaluate the mean error in $\mathrm{dB}$ between real data (from both master and slave images) and the derived backscatter models. If the $\beta_{u n W}^{0}(\theta)$ model, obtained by taking into account the $\beta^{0}$ unweighted mean values, is considered, one obtains:

$$
E\left[\Delta \beta_{u n W}^{0}\right]=E\left[\left|E\left[\boldsymbol{\beta}_{c}^{0}\right]-\beta_{u n W}^{0}\left(E\left[\boldsymbol{\theta}_{c}\right]\right)\right|\right],
$$

where index $c$ identifies either master $(c=m)$ or slave $(c=s)$ images.

By considering now the $\beta_{W}^{0}(\theta)$ model, derived from the $\beta^{0}$ weighted mean values only, one has:

$$
E\left[\Delta \beta_{W}^{0}\right]=E\left[\left|E\left[\boldsymbol{\beta}_{c}^{0}\right]-\beta_{W}^{0}\left(E\left[\boldsymbol{\theta}_{c}\right]\right)\right|\right] .
$$


Table 5.1.: Rain forest test sites for the validation of the closed to open $(>15 \%)$ broadleaved evergreen or semi-deciduous forest $(>5 \mathrm{~m}$ ) class (class 5 in Fig. 5.1). Acquisition parameters summary. $E\left[\theta_{m}\right]$ and $E\left[\theta_{s}\right]$ are the master and slave mean incidence angles, Master/slave Date are the master and slave images acquisition dates ( in year-month-day format), Orbit identifies the master/slave orbit direction (ASC: ascending, DESC: descending).

\begin{tabular}{ccccc}
\hline \hline Site & Location & $\boldsymbol{E}\left[\boldsymbol{\theta}_{m}\right] / \boldsymbol{E}\left[\boldsymbol{\theta}_{s}\right]$ & Master/slave Date & Master/slave Orbit \\
\hline & & deg & yyyy-mm-dd & \\
\hline T1 & Amazonas & $47.99 / 30.29$ & $2010-12-13 / 2011-09-08$ & DESC/DESC \\
\hline T2 & Amazonas & $48.13 / 30.26$ & $2010-12-21 / 2011-08-14$ & DESC/DESC \\
\hline T3 & Amazonas & $48.11 / 30.20$ & $2010-12-22 / 2011-08-26$ & DESC/DESC \\
\hline T4 & Amazonas & $43.56 / 43.55$ & $2011-01-09 / 2011-12-27$ & DESC/DESC \\
\hline T5 & Amazonas & $44.12 / 47.49$ & $2011-01-09 / 2012-01-10$ & DESC/ASC \\
\hline T6 & Amazonas & $38.67 / 36.09$ & $2011-05-04 / 2011-07-29$ & DESC/ASC \\
\hline T7 & Amazonas & $44.13 / 47.49$ & $2011-02-05 / 2012-01-04$ & DESC/ASC \\
\hline T8 & Amazonas & $47.44 / 44.22$ & $2010-12-12 / 2011-03-13$ & DESC/ASC \\
\hline T9 & Congo & $43.03 / 31.11$ & $2011-02-14 / 2012-03-25$ & DESC/ASC \\
\hline T10 & Congo & $47.77 / 44.15$ & $2010-12-13 / 2011-11-05$ & ASC/DESC \\
\hline
\end{tabular}

For the considered test sites, the mean differences are:

$$
\begin{array}{lll}
E\left[\Delta \beta_{u n W}^{0}\right]=0.48 & \mathrm{~dB} & \text { and } \\
E\left[\Delta \beta_{W}^{0}\right]=-0.05 & \mathrm{~dB}, & \text { respectively. }
\end{array}
$$

Such values show a significant improvement in the offset accuracy of the models, by using quality weights within the estimation process, as presented in section 5.3.2.

Curvature and slope accuracy. Fig. 5.14 presents the obtained results for the verification of the backscatter model slope/curvature accuracy. For each test site, described in Tab. 5.1, the mean $\beta^{0}$ of each master image (red rhombus) is depicted, together with the mean $\beta^{0}$ from the corresponding corrected slave image, evaluated as in Fig. 5.10 using $\beta_{\text {unW }}^{0}(\theta)$ models (yellow triangles) and $\beta_{W}^{0}(\theta)$ (green circles), respectively. $\beta^{0}$ 


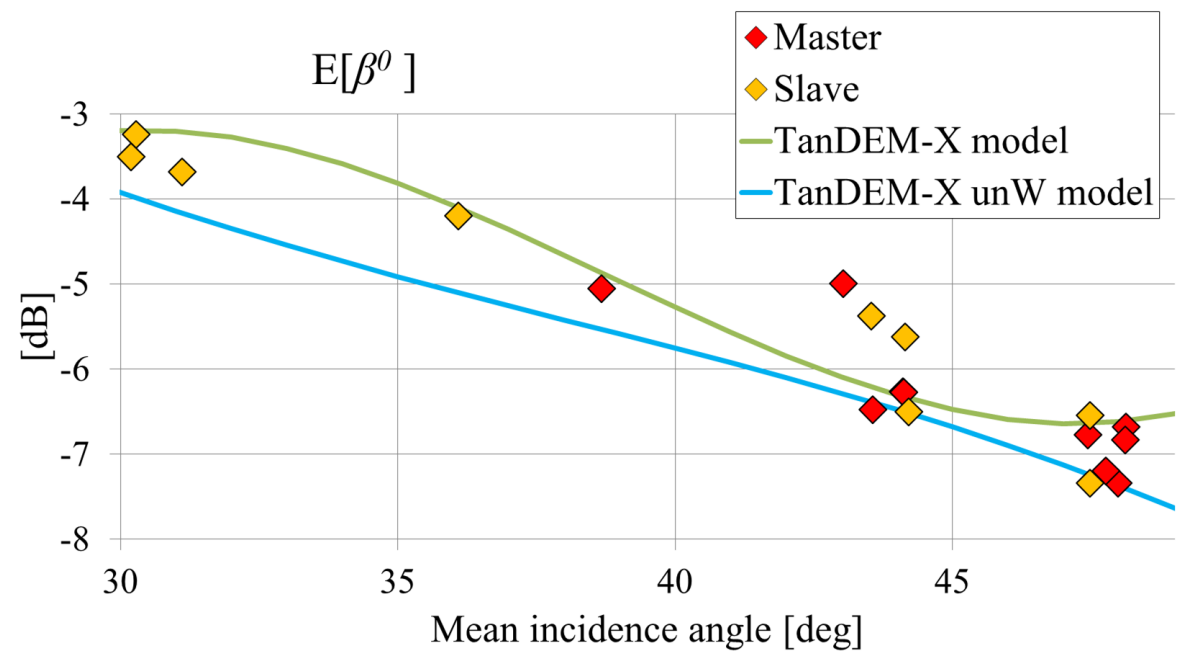

Figure 5.13.: Check for absolute offsets for the closed to open (>15\%) broadleaved evergreen or semi-deciduous forest (>5m) GlobCover class (class 5 in Fig. 5.1). For each considered test site in Tab. 5.1, the master and slave images mean radar brightness are displayed: $E\left[\boldsymbol{\beta}_{m}^{0}\right]$ (red rhombus) and $E\left[\boldsymbol{\beta}_{s}^{0}\right]$ (yellow rhombus), respectively. The bold lines identify the corresponding TanDEM-X backscatter model based on $\beta^{0}$ unweighted mean values (light blue) and the TanDEM-X backscatter model obtained taking into account the $\beta^{0}$ weighted mean values (green), as presented in section 5.3.2.

correction factors are directly evaluated and applied in logarithmic scale. One can now evaluate the mean backscatter difference between master and corrected slave as:

- $\Delta \beta_{m s}^{0}\left(E\left[\boldsymbol{\theta}_{m}\right], E\left[\boldsymbol{\theta}_{s}\right]\right)_{u n W}$ (triangles) takes into account slave images which are corrected to the corresponding master incidence angles by applying a correction factor $\delta \beta^{0}\left(\theta_{m}, \theta_{s}\right)_{u n W}$ obtained by taking into account the $\beta_{u n W}^{0}(\theta)$ models derived as presented in section 5.4.2, equation (5.14).

- $\Delta \beta_{m s}^{0}\left(E\left[\boldsymbol{\theta}_{m}\right], E\left[\boldsymbol{\theta}_{s}\right]\right)_{W}$ (circles) instead considers slave images corrected to the corresponding master incidence angles by applying a correction factor $\delta \beta^{0}\left(\theta_{m}, \theta_{s}\right)_{W}$ obtained by taking into account the $\beta_{W}^{0}(\theta)$ models derived as presented in section 5.4.2, equation (5.25).

On the horizontal axis, the mean incidence angle difference $\Delta \theta_{m s}$ between the master and the corresponding slave acquisition is displayed.

The root mean square error between master and corrected slave images (evaluated in logarithmic scale) is: $R M S E_{W}=0.8 \mathrm{~dB}$ and $R M S E_{u n W}=0.6 \mathrm{~dB}$ for correction curves derived from weighted and unweighted $\beta^{0}$ models, respectively. 


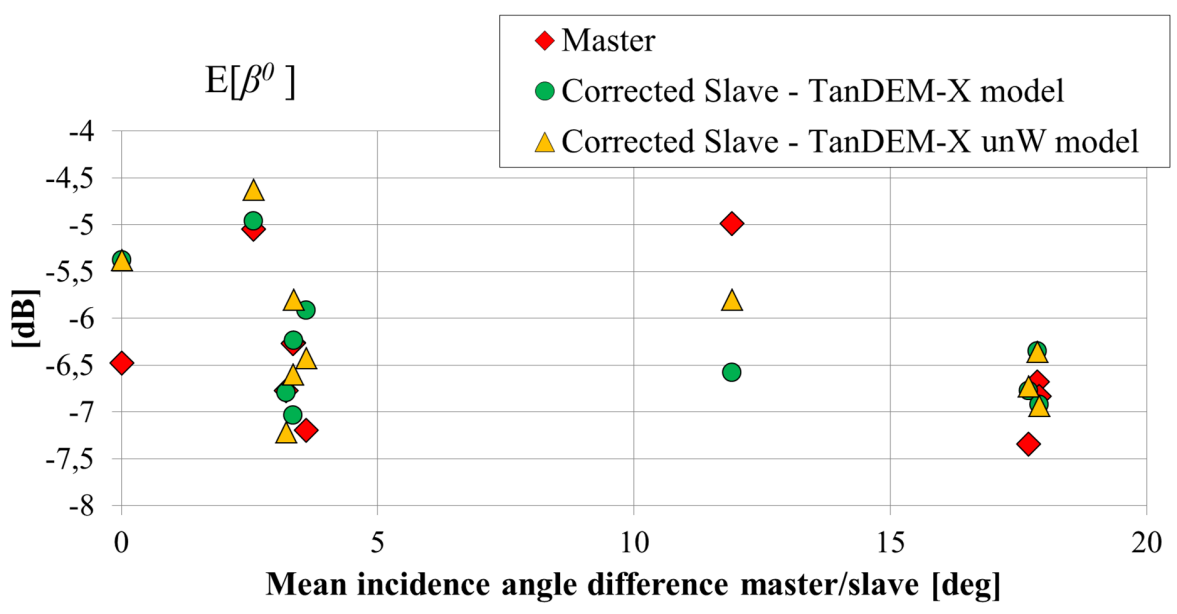

Figure 5.14.: Slope/curvature accuracy of the derived backscatter models for the closed to open $(>15 \%)$ broadleaved evergreen or semi-deciduous forest $(>5 \mathrm{~m})$ GlobCover class (class 5 in Fig. 5.1). Red rhombus: mean $\beta^{0}$ of each master image, green circles and yellow triangles: mean $\beta^{0}$ of the corrected slave images using $\beta_{W}^{0}(\theta)$ and $\beta_{u n W}^{0}(\theta)$ models, respectively. The horizontal axis displays the mean incidence angle difference $\Delta \theta_{m s}$ between the master and the corresponding slave acquisition.

Overall, for the Closed to open (>15\%) broadleaved evergreen or semi-deciduous forest $(>5 \mathrm{~m})$ class, results show that the derived $\beta^{0}$ weighted model presents a better performance compared to the unweighted one in terms of remaining absolute offsets, while the unweighted one performs slightly better when evaluating the model curvature/slope, showing an overall RMSE which is $0.2 \mathrm{~dB}$ lower. Both offset and curvature/slope accuracy are below $1 \mathrm{~dB}$.

\subsection{Seasonal and Geographic Backscatter Dependency}

Radar backscatter can behave differently over the year, depending on when a SAR acquisition is performed. Its intensity is influenced by several factors, such as soil moisture or ground conditions, whose average trends can be related to a particular season of acquisition.

For example, SAR acquisitions over temperate forests during winter, when trees typically do not have leaves, or during summer, lead to different levels of backscatter. On the contrary, if regions around the Equator are considered, seasonal changes are not so relevant.

Hence, it is important to take these factors into account for the generation of a reliable set of backscatter models, starting from a global data base of SAR images, acquired during 
different seasons.

In order to mitigate such effects, the developed backscatter modeling technique can be separately applied for different seasons, allowing for a better temporal refinement of the derived mean $\beta^{0}$ models.

To do so, the complete data base of TanDEM-X input images can be split into different groups, depending on the season of acquisition. For example, if the boreal hemisphere calender is considered as reference, winter and summer can be associated to the time spans which go from December to February and from May to September, respectively. Moreover, seasons in boreal and austral hemispheres have to be correctly grouped together as well. Therefore, if a first image, acquired over Europe in January, and second one, acquired over South America in July, are considered, they will be both associated to the winter season. In this way, the impact of seasonality on the final estimation accuracy is considerably reduced.

An example is presented in Fig. 5.15, where two different GlobCover classes are considered: Rainfed Croplands (class 2 in Fig. 5.1) (a) and Closed to open (>15\%) broadleaved evergreen or semi-deciduous forest ( $>5 \mathrm{~m}$ ) (class 5 in Fig. 5.1) (b). Two different seasonal models have been estimated, depending on the season of the input acquisitions. Class 2 identifies farming practices that rely on rainfall for water supply and is therefore strongly related to seasonal precipitation. In this case, significant offsets can be detected between winter and summer mean $\beta^{0}$ models, with differences up to about $3 \mathrm{~dB}$. On the other hand, class 5 identifies typical rainforests and is mostly situated around the Equator, where seasonal changes are not so intense. Here, offsets below $1 \mathrm{~dB}$ can be detected between winter and summer mean $\beta^{0}$ models.

Moreover, if a global classification map such as GlobCover is used, one should note that the same classification class can typically be spread almost all over the entire planet. Tree species, local weather, and ground conditions, as well as temperatures and soil moisture, can significantly change from one region to another, leading again to different backscatter behaviors. Also in this case, input $\beta^{0}$ images can be further grouped together at a continent scale, leading to the generation of continental backscatter models.

This approach was used for the generation of the data base of backscatter models presented in Appendix A. Here, mean $\beta^{0}$ weighted models are derived for each GlobCover class, separately. For the sake of simplicity, for each class, backscatter models for a single continent are provided. The continent's choice was based on the number of available input observations and on how representative such a continent is for the considered land cover type. 


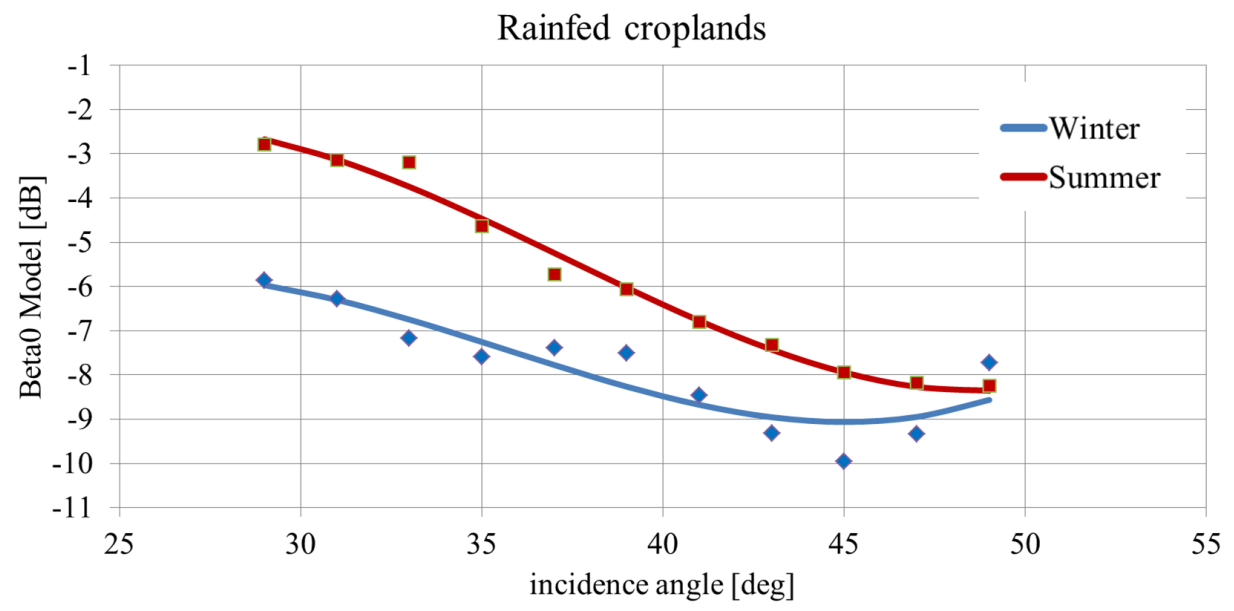

(a)

Closed to open $(>15 \%)$ broadleaved evergreen or semi-deciduous forest $(>5 \mathrm{~m})$

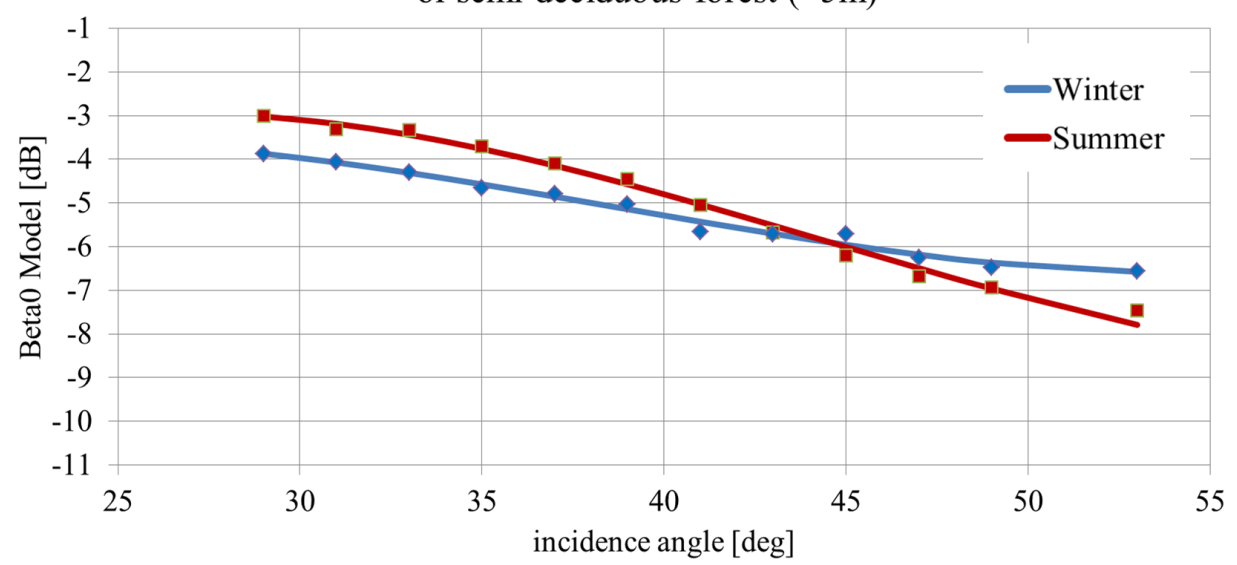

(b)

Figure 5.15.: Estimated weighted mean $\beta^{0}$ models for the GlobCover classes Rainfed Croplands (class 2 in Fig. 5.1) (a) and Closed to open (>15\%) broadleaved evergreen or semi-deciduous forest $(>5 \mathrm{~m})$ (class 5 in Fig. 5.1) (b). Winter models: blue solid line, Summer models: red solid line. The derived weighted mean $\beta^{0}$ values for each incidence angle interval are displayed with marks. 


\subsection{A Large-Scale Study of Tropical Rainforests Backscatter}

The radiometric calibration of spaceborne SAR system is a critical factor for assuring a good performance of the delivered products. For example, a key quantity for performing such a task is the knowledge of the antenna pattern, which is normally characterized by using on-ground measurements in the pre-launch phase. The antenna absolute calibration is then performed after the launch by using reference targets, such as transponders and corner reflectors. The increased complexity of steerable antennas makes it inevitable to verify and monitor the pattern shape through the in-flight phase as well. Tropical rainforests, such as the Amazon and Congo forests, have been established by the SAR community as a useful means for estimating the elevation antenna pattern variations, as explained in [106] and [101]. The homogenous signature of rainforests is used as a reference to derive the pattern shape from the measured backscatter. The assumption is that the considered ground area is isotropic and that its average radar cross-section for a given acquisition geometry and time of year is known.

Nevertheless, several studies have shown a slight dependency of the rainforest backscatter on the incidence angle, e. g. by taking into account the ASCAT C-band scatterometer [107] or the RADARSAT-2 C-band SAR sensor [108]. Moreover, the backscattering properties of the rainforest are influenced by ground target properties and meteorological conditions as well. In particular, they can be influenced by the precipitation occurrence, which impact on the level of humidity on ground, as presented in [109] and [110].

The backscatter mapping quantity used for calibration purposes is the backscattering coefficient $\gamma^{0}$, derived as in (2.46). Given the hypothesis of an isotropic scatterer, the $\gamma^{0}$ profile over incidence angle is flat.

The proposed analysis concentrates on the investigation of homogeneous tropical forests located in different areas using TanDEM-X data. Moreover, ascending (ASC) and descending (DESC) orbit acquisitions are available as well, allowing for a characterization of $\gamma^{0}$ depending on the day time of acquisition, as they are typically acquired during the evening or morning, respectively ${ }^{2}$. Finally, given the time span of the available data, operationally acquired within the TanDEM-X mission since December 2010, the aim of this analysis is to analyze the seasonal variation of X-band backscatter over tropical rainforests as well, by looking at time series of data which cover a temporal interval of about three years.

The current analysis over tropical rainforests has been developed in the frame of the CEOS SAR Calibration and Validation Working Group activities, and published in [111].

\footnotetext{
${ }^{2}$ TanDEM-X is operationally acquiring the northern hemisphere in ASC orbit direction, and the southern hemisphere in DESC, using stripmap mode in single HH polarization.
} 


\subsubsection{Input Test Sites and $\gamma^{0}$ Quicklook Mosaics}

Two different test sites have been taken into account for the current analysis: situated in Amazonas and in the Democratic Republic of the Congo, respectively. The Amazonas test site (Fig. 5.16 (a)) extends over $9^{\circ} \times 6^{\circ}$ in latitude/longitudes coordinates, while the Democratic Republic of the Congo one (Fig. 5.16 (b)) extends over $2^{\circ} \times 2^{\circ}$.

Both test sites cross the Equator, which means that images acquired in both ASC and DESC orbit direction, corresponding to evening and morning acquisitions, respectively, are available. All considered images were acquired in single $\mathrm{HH}$ polarization, with incidence angles between $31^{\circ}$ and $48^{\circ}$, over a time span of three years (from 2011 up to 2013).

Single quicklook images of $\gamma^{0}$ and the corresponding incidence angle $\theta$ were mosaicked together, at a final resolution of $100 \mathrm{~m} \times 100 \mathrm{~m}$. Several mosaics were generated, depending on the purpose of the investigation, i.e. by composing images acquired during a particular season of the year or acquired with a certain orbit direction only. As an example, a zoom of a $\gamma^{0}$ mosaic over the Amazonas, generated using ASC acquisitions only, is presented in Fig. 5.16 (c).

\subsubsection{Analysis of $\gamma^{0}$ Incidence Angle Dependency}

In this section, $\gamma^{0}$ behavior is analyzed with respect to the incidence angle of illumination. In particular, a set of incidence angle intervals is considered, each of them evaluated at the center of each operational TanDEM-X beam, considering an angular span of $1^{\circ}$ (from about $31^{\circ}$ to $48^{\circ}$ ). $\gamma^{0}$ samples from the considered quicklook mosaic, belonging to each single interval, are grouped together, allowing for the derivation of a mean $\gamma^{0}$ value for each interval.

The obtained results for the Amazonas test site are presented in Fig. 5.17. Fig. 5.17 (a) shows two $\gamma^{0}$ mosaics, separately generated using ASC and DESC acquisitions, respectively. On the right-hand side (Fig. 5.17 (b)), the corresponding incidence angles of illumination are depicted. It is worth noting that the full nominal TanDEM-X beam coverage, composed by nine adjacent beams, has to be used to acquire such an area without gaps. Since the next (neighbor) acquisitions can only be performed after a complete orbit cycle of 11 days, more than a three months period is required to achieve the complete coverage.

The mean values of $\gamma^{0}$ per each different incidence angles interval are depicted in Fig. 5.17 (c). Mean $\gamma^{0}$ from ASC acquisitions (mean value of $-6.28 \mathrm{~dB}$, standard deviation of $0.15 \mathrm{~dB}$ ) appears to be more stable with respect to the DESC ones (mean values of 


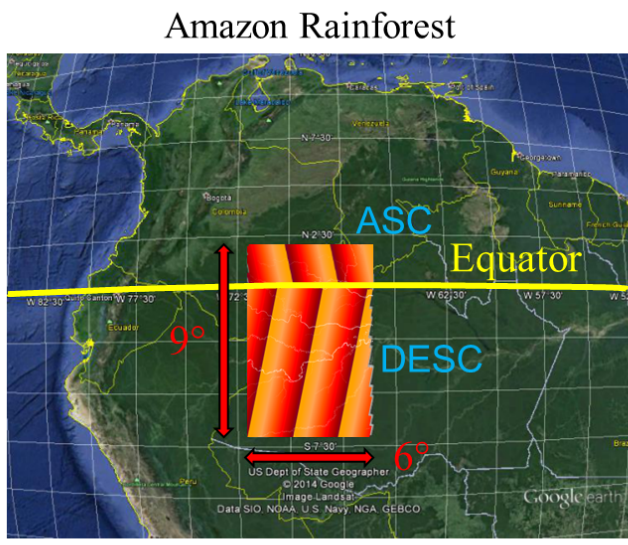

(a)

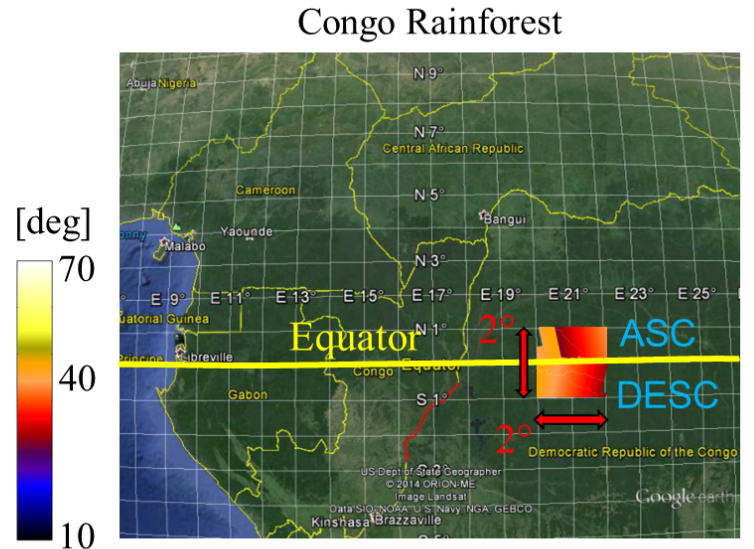

(b)

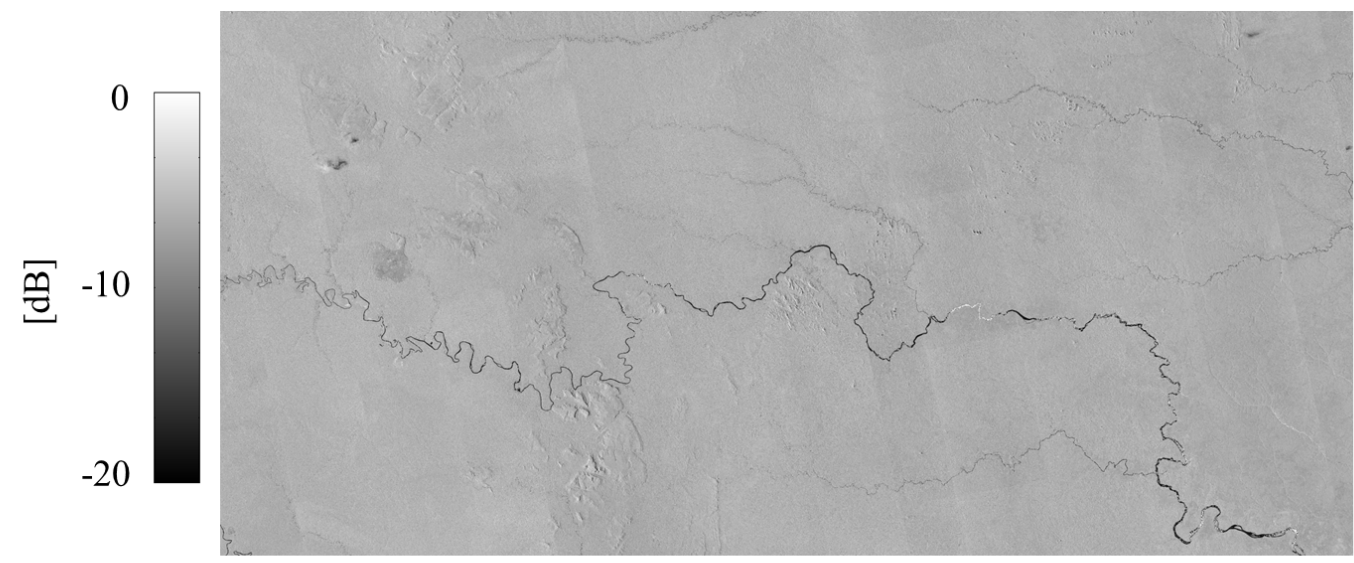

(c)

Figure 5.16.: Test Sites used for the large-scale study of tropical rainforests: (a) Amazonas (extending by $9^{\circ} \times 6^{\circ}$ in latitude/longitudes coordinates), (b) Democratic Republic of the Congo (extending by $2^{\circ} \times 2^{\circ}$ in latitude/longitudes coordinates). Mosaics of the incidence angle for the available acquisitions are displayed. The northern hemisphere is acquired in ascending orbit direction (ASC), while the southern hemisphere in descending one (DESC). (c) Zoom in of a $\gamma^{0}$ mosaic over the Amazon rainforest, generated using ASC acquisitions only.

$-5.89 \mathrm{~dB}$, standard deviation of $0.32 \mathrm{~dB}$ ). Moreover, especially at near range, DESC acquisitions are characterized by higher backscatter levels than ASC ones, showing a slight decrease with the increase of the incidence angle.

The same analysis can be carried out by considering the overlapping region between ASC and DESC acquisitions, as shown in Fig. 5.18. In this way, the same target area is 


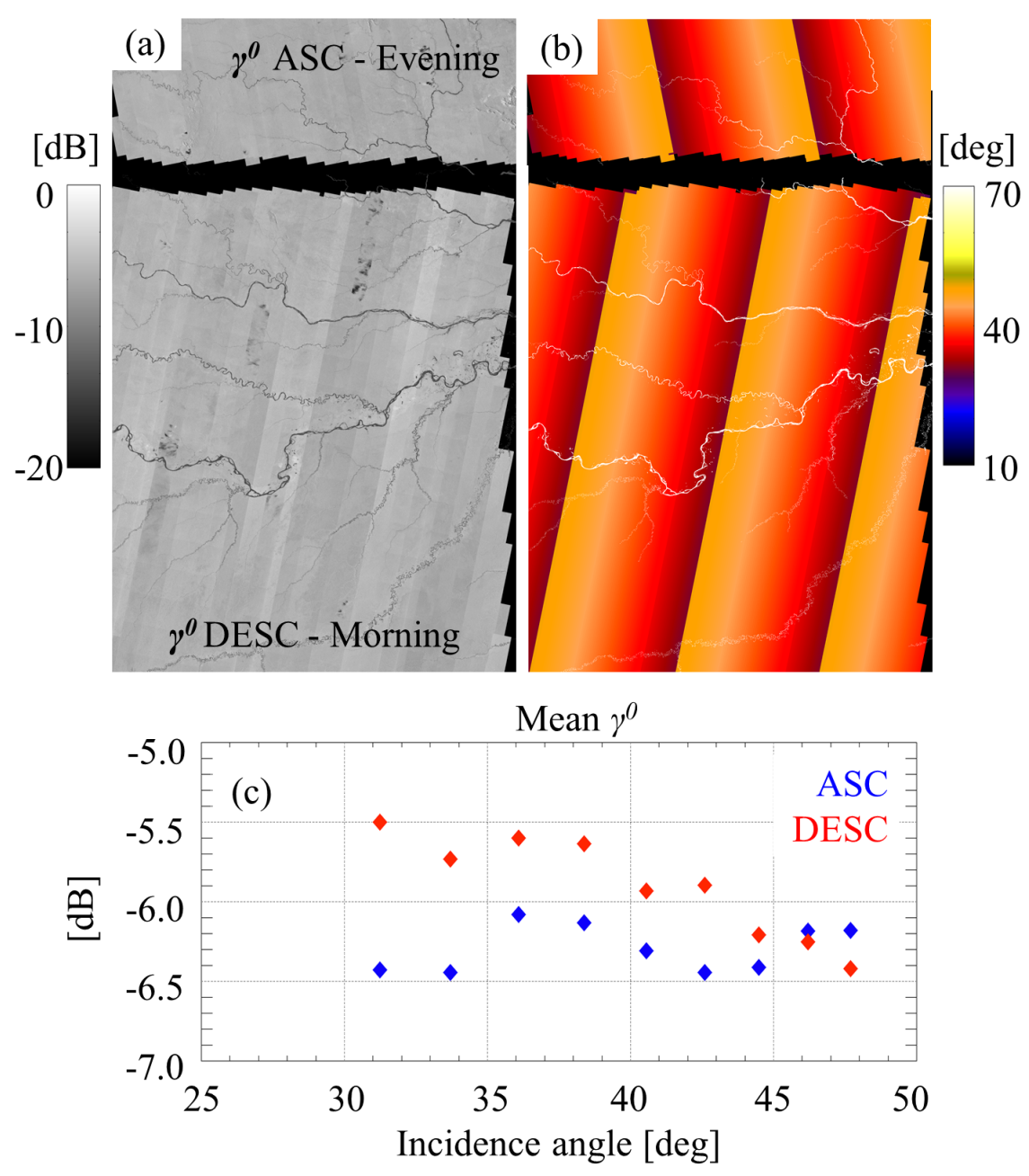

Figure 5.17.: (a) $\gamma^{0}$ mosaic of ascending and descending TanDEM-X acquisitions over the Amazon rainforest. The region of interest is defined as follows: latitudes between $-7^{\circ}$ and $2^{\circ}$, longitudes between $-72^{\circ}$ and $-66^{\circ}$. (b) Corresponding incidence angles. (c) $\gamma^{0}$ dependency on the incidence angle.

considered for both ASC and DESC acquisitions, reducing the impact of different target properties. Also in this case, it can bee seen that DESC acquisitions, performed in the morning, show higher levels of $\gamma^{0}$ at near range.

The same quantities as in Fig. 5.17 are presented in Fig. 5.19 for the Congo rainforest test site. All used images were acquired during the first year of TanDEM-X operation (2011). The mean $\gamma^{0}$ from ASC and DESC acquisitions present a mean value of -6.21 $\mathrm{dB}$ and $-5.91 \mathrm{~dB}$, respectively. The standard deviation in ASC orbit mode $(0.16 \mathrm{~dB})$ is 
(a) - $\gamma^{0}$ ASC - Evening

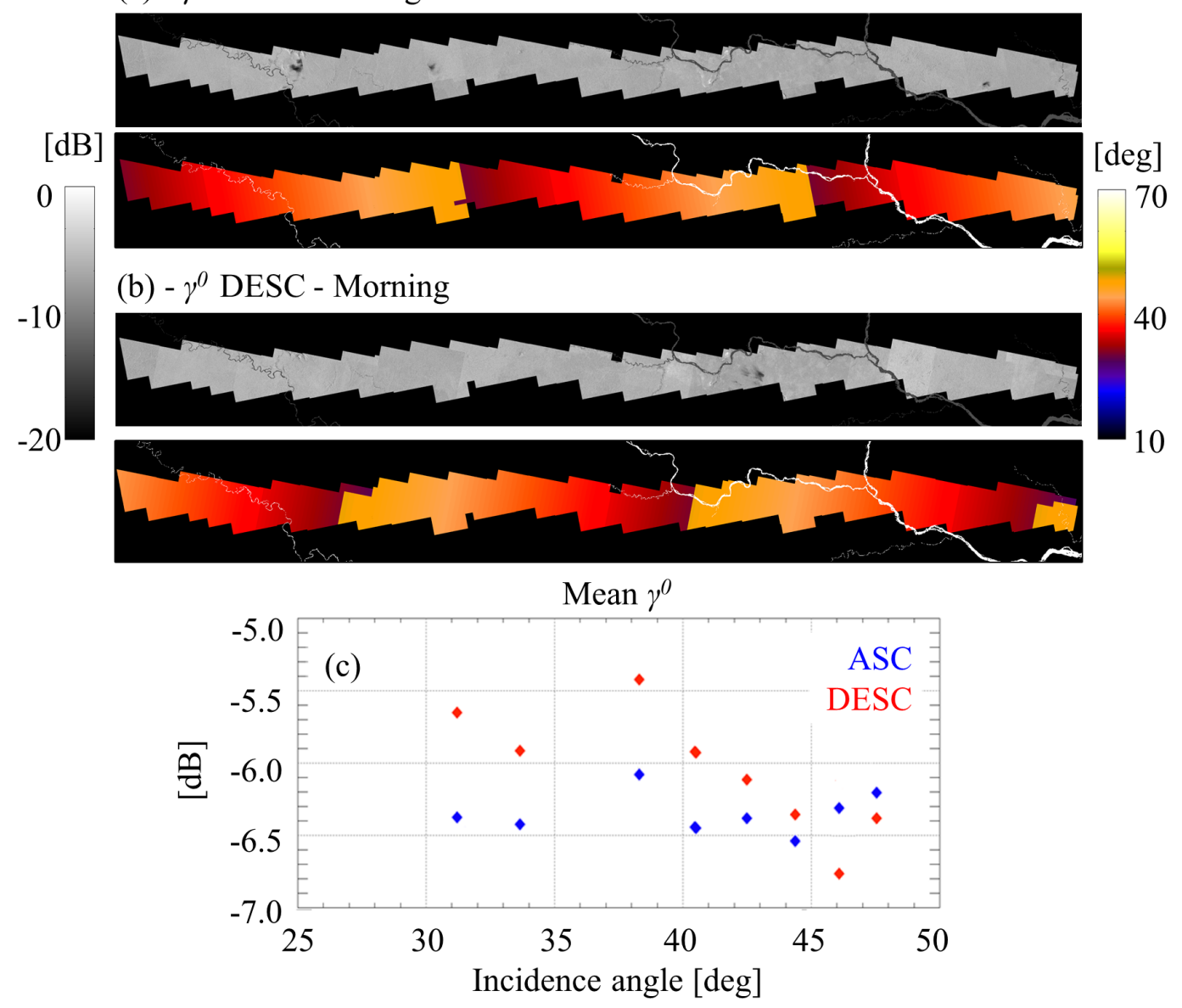

Figure 5.18.: $\gamma^{0}$ mosaics over the overlapping area for ascending (a) and descending (b) TanDEM-X acquisitions over the Amazon rainforest, together with the corresponding incidence angles. (c) $\gamma^{0}$ dependency on the incidence angle.

lower than in the DESC one $(0.35 \mathrm{~dB})$.

\subsubsection{Analysis of $\gamma^{0}$ Seasonal Dependency}

An analysis of $\gamma^{0}$ dependency on the seasonal acquisition period has been performed over the Amazon rainforest test site only, given the availability of data over a time span of three years. Different mosaics were generated, whose input data were acquired in predefined time intervals only. In particular, separate $\gamma^{0}$ mosaics were generated for 


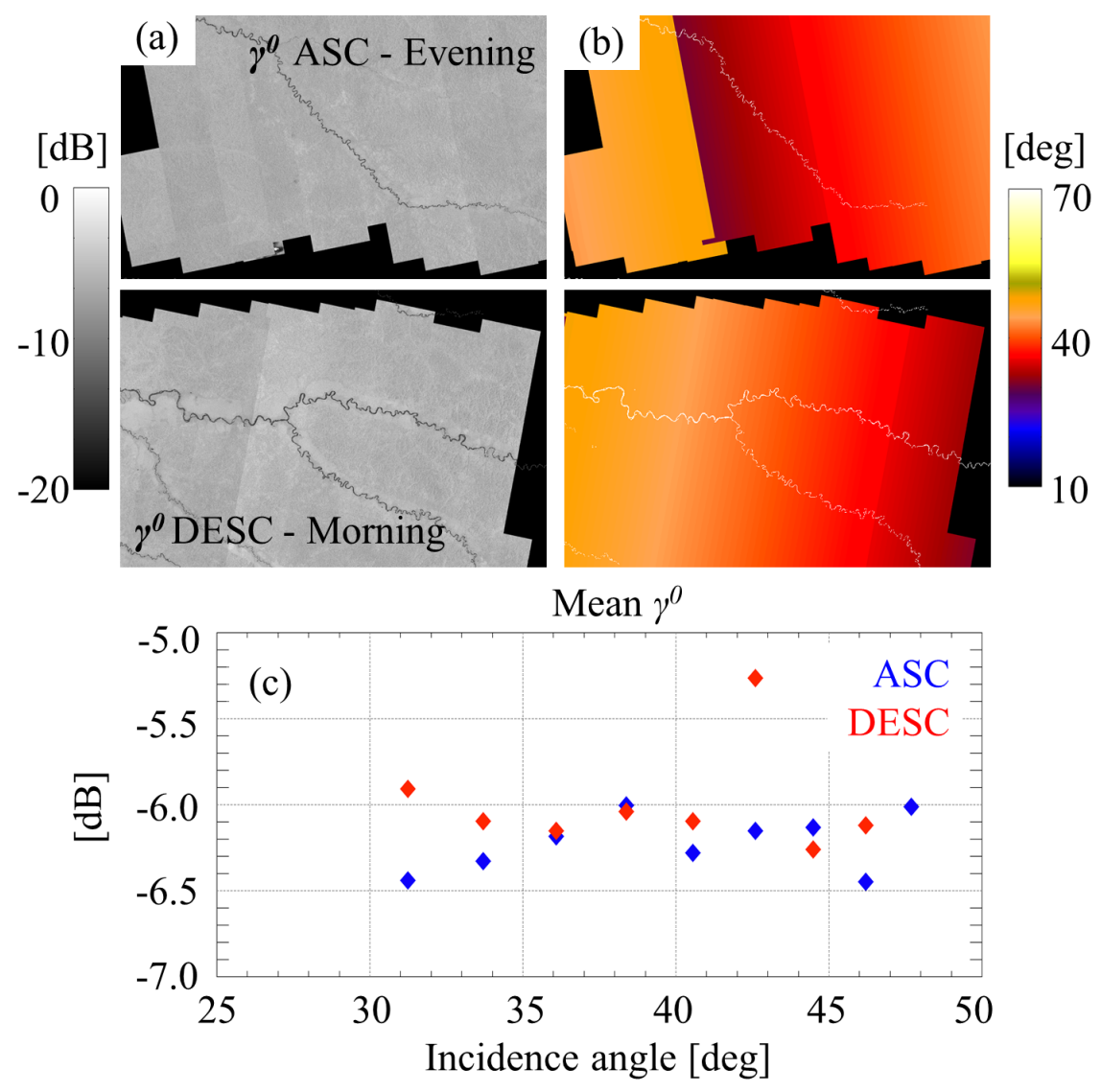

Figure 5.19.: (a) $\gamma^{0}$ mosaics of ascending and descending TanDEM-X acquisitions over the Congo rainforest (acquired over the Democratic Republic of the Congo). The region of interest is defined as follows: latitudes between $-1^{\circ}$ and $1^{\circ}$, longitudes between $20^{\circ}$ and $22^{\circ}$. (b) Corresponding incidence angles. (c) $\gamma^{0}$ dependency on the incidence angle.

summer and winter for the years 2011,2012, and 2013. A summary of the considered acquisition periods is presented in Tab. 5.2.

An example of seasonal mosaics is depicted in Fig. 5.20 (a)-(d), where $\gamma^{0}$ mosaics from TanDEM-X ASC/DESC acquisitions over the Amazon rainforest for summer 20112013 ((a)-(b)) and winter 2011-2013 ((c)-(b)) are shown. As it can be seen, the considered acquisitions do not cover the entire range of TanDEM-X incidence angles in a single season. Therefore, a three years time period has been considered, by grouping together mean $\gamma^{0}$ values coming from either summer or winter mosaics of 2011, 2012, and 2013. 


\begin{tabular}{lcccc}
\hline \hline & \multicolumn{3}{c}{ Seasonal Mosaics Summary } \\
\hline Year & \multicolumn{2}{c}{ Summer } & \multicolumn{2}{c}{ Winter } \\
& Start Date & End Date & Start Date & End Date \\
\hline 2011 & $1^{\text {st }}$ Jun. 2011 & $31^{\text {st }}$ Aug. 2011 & $1^{\text {st }}$ Dec. 2010 & $28^{\text {th }}$ Feb. 2011 \\
\hline 2012 & $1^{\text {st }}$ Jun. 2012 & $31^{\text {st }}$ Aug. 2012 & $1^{\text {st }}$ Dec. 2011 & $28^{\text {th }}$ Feb. 2012 \\
\hline 2013 & $1^{\text {st }}$ Jun. 2013 & $31^{\text {st }}$ Aug. 2013 & $1^{\text {st }}$ Dec. 2012 & $28^{\text {th }}$ Feb. 2013 \\
\hline
\end{tabular}

Table 5.2.: Summary of the acquisition periods taken into account for the generation of seasonal $\gamma^{0}$ mosaics.

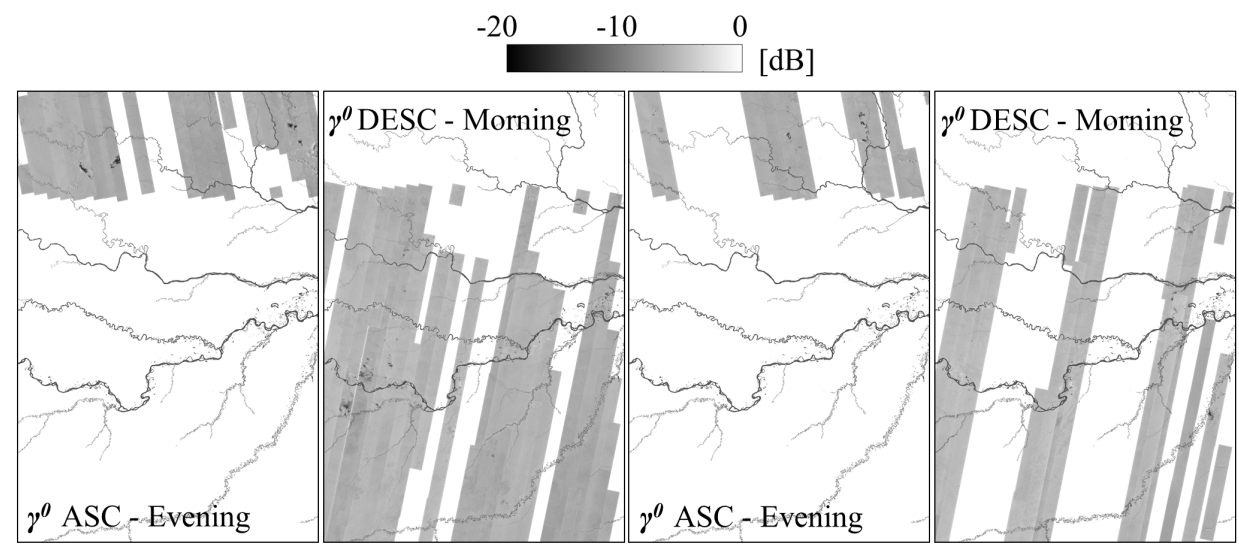

(a)

(b)

(c)

(d)

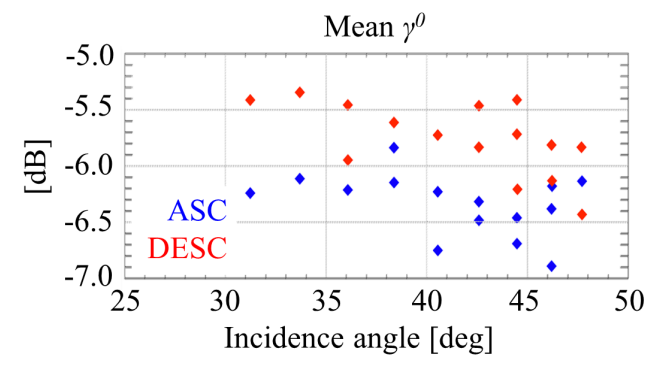

(e)

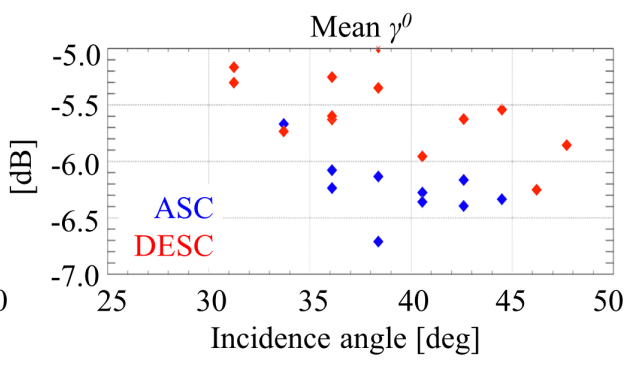

(f)

Figure 5.20.: Seasonal behavior of $\gamma^{0}$ over the Amazon rainforest. (a) and (b) Mosaics of $\gamma^{0}$ from acquisitions acquired from 2011 to 2013 in the months of June, July, and August ((a) ASC, (b) DESC). (c) and (d) Mosaics of $\gamma^{0}$ from acquisitions acquired from 2011 to 2013 in the months of December, January, and February. (e) $\gamma^{0}$ mean values from ASC (blue) and DESC (red) acquisitions, acquired from 2011 to 2013 in the months of June, July, and August. (f) $\gamma^{0}$ mean values from ASC (blue) and DESC (red) acquisitions, acquired from 2011 to 2013 in the months of December, January, and February. 
The obtained results are presented in Fig. 5.20 ((e)-(f)), showing the overall mean $\gamma^{0}$ for summer and winter, obtained by combining all available $\gamma^{0}$ seasonal mosaics from ASC and DESC acquisitions, respectively.

For both summer and winter, mean $\gamma^{0}$ values from DESC acquisitions appear overall brighter than the ones obtained from ASC acquisitions, showing an overall offset of about $0.5 \mathrm{~dB}$. Nevertheless, no significant difference between the two seasons behavior is detectable.

From the performed analysis one can therefore conclude that rainforest backscatter in $\mathrm{X}$ band is mostly influenced by the day time of acquisition, rather than by seasonal effects. Important factors which can explain such a behavior are the level of humidity of the air or the presence of water on the tree leaves, which strongly increase during the night time and whose effects are therefore clearly visible in acquisitions performed during the morning. 


\section{The Greenland Ice Sheet Case}

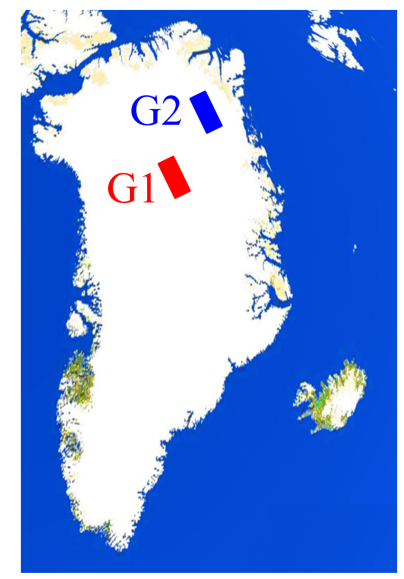

(a)

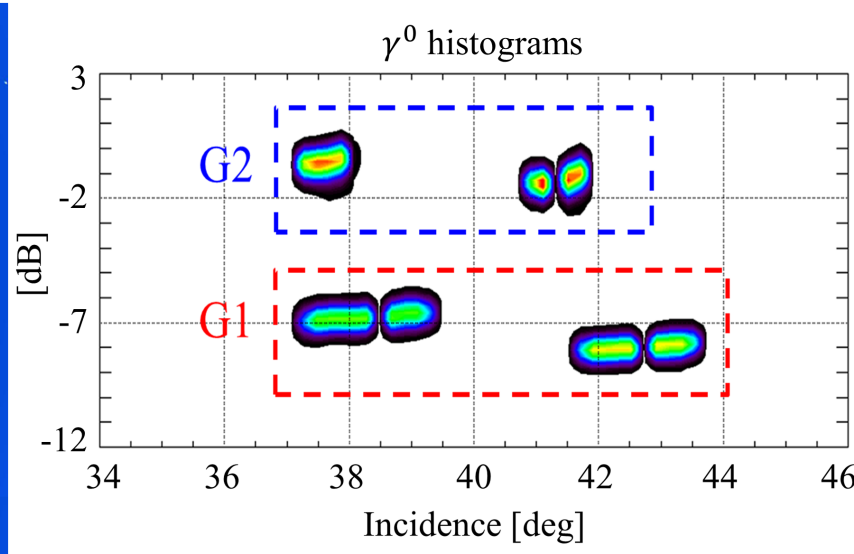

(b)

Figure 6.1.: (a) GlobCover classification Map over Greenland (white: Permanent snow and ice class). G1 and G2 identify two test sites where TanDEM-X acquisitions in $\mathrm{HH}$ polarization were performed. The corresponding histograms of $\gamma^{0}$ are depicted in (b).

The Greenland Ice Sheet is characterized by significant spatial variations of backscatter returns, as detailed in section 6.1. Nevertheless, only one single classification class is provided by the GlobCover map, as shown in Fig. 6.1 (a). Here, two test sites (G1 and G2) are superimposed to the GlobCover map, where TanDEM-X images in HH polarization were acquired during the winter season. The derived histograms of $\gamma^{0}$ for such acquisitions are shown in Figure 6.1 (b). Differences in backscatter levels between the two test sites of more than $5 \mathrm{~dB}$ are detected.

It is therefore not possible to model the backscatter behavior from the entire Greenland Ice Sheet, using the approach presented in chapter 5, since the GlobCover classification map does not provide a sufficient level of detail.

\section{The need for a better classification let to the work presented in this chapter.}

Here, a detailed study on information content of X-band single-pass interferometric spaceborne SAR data with respect to snow facies characterization is presented. 
An approach for classifying different snow facies of the Greenland Ice Sheet by exploiting X-band TanDEM-X interferometric synthetic aperture radar acquisitions is firstly detailed. Large-scale mosaics of radar backscatter and volume correlation factor, derived from quicklook images of the interferometric coherence, represent the starting point for applying an unsupervised classification method based on the $c$-means fuzzy clustering algorithm. The used data were acquired during winter 2010/2011.

A partition of four different snow facies was chosen and interpreted using reference melt data, snow density, and in situ measurements. The variations in the stratification and micro-structure of firn, such as the variations of density with depth and the presence of percolation features, are identified as relevant parameters for explaining the significant differences in the observed interferometric signatures among different snow facies. Moreover, a statistical analysis of backscatter and volume correlation factor provided useful parameters for characterizing the snow facies behavior and analyzing their dependency on the acquisition geometry.

Afterwards, two applications of the derived snow facies are presented:

- In section 6.6.1, the derived snow facies map is used as reference classification for applying the backscatter modeling algorithm presented in chapter 5 .

- In section 6.6.2, the two-way X-band penetration depth over the whole Ice Sheet is estimated. The obtained mean values vary from $2.3 \mathrm{~m}$ for the outer snow facies up to $4.18 \mathrm{~m}$ for the inner one.

The presented approach represents a starting point for a long-term monitoring of ice sheet dynamics, by acquiring time-series, and is of high relevance for the design of future SAR missions as well.

The developed algorithms for locating different snow facies (sections 6.1 to 6.5) and estimating the penetration depth into the Ice Sheet (section 6.6.2) were published in [112].

\subsection{The Greenland Ice Sheet: an Introduction to the Work}

The Greenland Ice Sheet, extending by about $1,700,000 \mathrm{~km}^{2}$ and covering over $80 \%$ of the entire Greenland surface, represents the second largest ice body on the planet after the Antarctic Ice Sheet. Its properties are significantly affected by temperature changes. Their knowledge can substantially contribute to a better understanding of the arctic and its response to climate change. Melt phenomena have strongly increased in the last years, therefore leading to modifications in the characteristics of the snow pack [113]. 


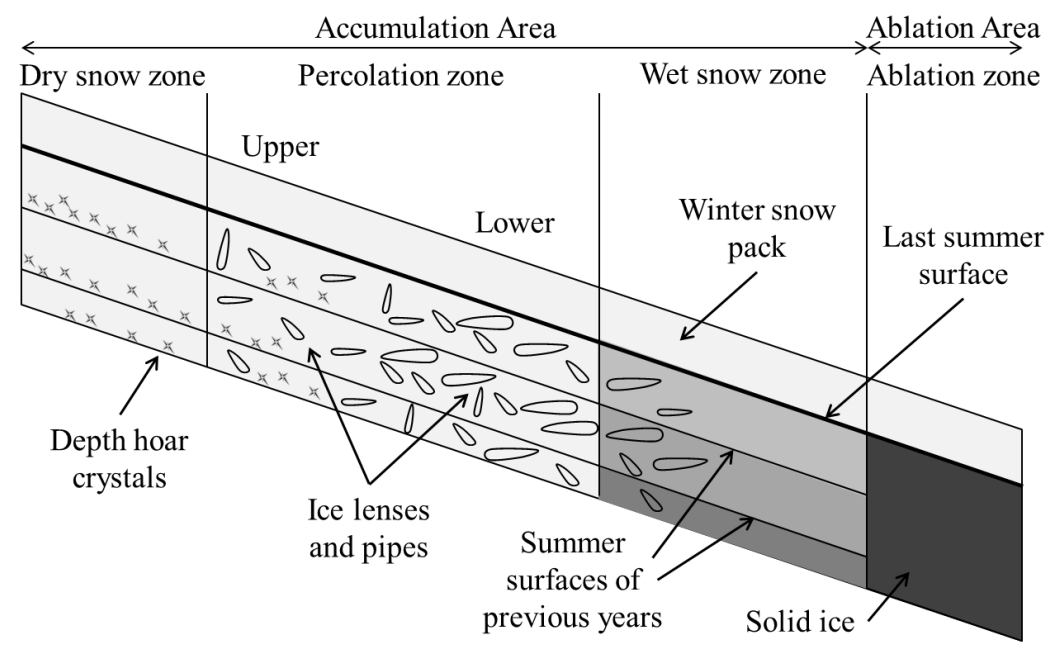

Figure 6.2.: Greenland Ice Sheet facies, classified after C. S. Benson in [103]: the dry snow zone, where no melt occurs, the percolation zone, where a limited amount of melt per year occurs and meltwater percolates and then refreezes within the snow pack, the wet snow zone, where a substantial part of the snow melt drains off during summer, and the ablation zone, where the previous year accumulation completely melts during summer.

Previous studies of the Greenland Ice Sheet led to the definition of different snow facies, depending on the amount of snow melt and on the properties of the snow coverage itself. Using a large number of survey sites, C. S. Benson [103] divided the Ice Sheet into four zones, according to Fig. 6.2. Melt does not occur in the dry snow zone, which is situated at the highest altitudes at the center of the Greenland plateau. The snow is gradually compacted under its own weight and the surface layer is subject to modifications due to wind effects. Moreover, the properties of the dry snow zone are not uniform, since it is characterized by different levels of snow accumulations, systematically decreasing from the southwest to the northeast regions of Greenland $[114,115]$. This inner region is surrounded by the percolation zone, where a limited amount of melt per year occurs, leading to the generation of larger snow grains and to the formation of small ice structures, like lenses and pipes, within the snow pack. The size of such ice formations can vary from some centimeters to tens of centimeters [116]. The wet snow zone is located further down slope towards Greenland's coasts, where a substantial part of the snow melt drains off during summer, and is characterized by the presence of multiple ice layers. Outer coastal regions are finally classified as ablation zone, where the previous year accumulation completely melts during summer, resulting in a surface of bare ice and surface 
moraine. Up to now, the different facies have been located using microwave sensors by estimating the backscatter levels of the reflected signal $[88,117]$. The dry snow zone is characterized by low levels of backscatter, given the absorption of the incident radar wave; on the other hand, the presence of ice pipes and lenses in the percolation snow, whose dimensions are comparable to the wavelength of the incident radar wave, strongly increase the backscattered signal from such a region. The scattering mechanisms occurring in the wet snow zone are similar to those in the percolation zone, even though a higher variability is expected during summer, due to increased melt rates [116]. Moreover, the availability of several spaceborne SAR missions allows for the monitoring of radar backscatter evolution in time, demonstrating the great potential of radar to track down changes in the Ice Sheet properties [118].

The penetration of an incident radar wave on a snow pack is dependent on the sensor's frequency and wave polarization, and on the characteristics of the illuminated target, such as snow density and structure, causing volume scattering. Interferometric synthetic aperture radar (SAR) acquisitions over the Greenland Ice Sheet are therefore subjected to volume decorrelation [119]. Its amount can be associated to the dominant backscattering mechanism for radar waves incident onto a snow pack, helping to classify the characteristics and structure of the snow pack itself.

The goal of classification techniques is to group together the input data in different classes on the basis of a defined measure of similarity. The approach can either be supervised, if a priori knowledge is introduced for defining the properties of the different classes, or unsupervised, if such classes are directly estimated from the input data, without external additional information. Since only a few local studies have been performed for determining the properties of the Greenland and Antarctica Ice Sheets using X-band SAR data [120,121, 122], only a limited a priori knowledge is available for directly defining the characteristics of each snow facies from X-band signatures. Unsupervised classification techniques, such as fuzzy clustering, therefore represent an attractive alternative.

A first preliminary study on the potential of TanDEM-X interferometric data for snow facies analysis using fuzzy clustering was presented in [123]. Here the classification algorithm is refined, its performance investigated in detail, and comparisons with in situ observations are performed to support interpretation of the results.

\subsection{Fuzzy Clustering for Snow Facies Classification}

In this section, the method used to classify the different snow facies of the Greenland Ice Sheet is described. It is based on the use of the $c$-means fuzzy clustering algorithm, developed by J. Bezdek et al. in [124], which is an unsupervised classification algorithm based on fuzzy logic theory.

Two characterizing radar quantities are considered for classifying snow facies: radar 
backscatter and volume correlation factor, which represents the coherence contribution due to volume decorrelation. The choice of an unsupervised classification method resides in the fact that a gradual transition of backscattering intensity between different snow facies on the Greenland Ice Sheet was observed by K. C. Partington in [125], impairing the use of a manual partitioning approach, which would strongly depend on the subjective choice of the decision thresholds. Moreover, the $c$-means fuzzy clustering algorithm has already been used in the literature for discriminating snow facies using Envisat active and passive microwave observations, showing it to be a promising approach for clustering similar regions of the Greenland Ice Sheet, as presented by Tran et al. in [126].

\subsubsection{The c-Means Fuzzy Clustering Optimization}

Clustering defines the task of grouping together elements coming from an input set of observations, depending on how similar they are to each other. The observations are divided into $c$ non-empty subsets called clusters. Since in reality clusters may show some kind of overlap, fuzzy clustering has been introduced [127]. The fuzzy $c$-means clustering algorithm is an iterative optimization algorithm which allows the determination of the optimal cluster centers without requiring a priori information [128]. In literature, the fuzzy $c$-means clustering has been found to be very popular within the research community, being used for a large variety of applications, such as risk and claim classification or vehicular pollution estimation [129, 130].

The idea is to represent the similarity that an observation shares with each cluster by using a membership function, whose values are between $0(0 \%$ probability of belonging to cluster $i$ ) and 1 (100\% probability of belonging to cluster $i)$. The results are fuzzy $c$-partitions of the input observation data set, which contain observations characterized by a high intracluster similarity and a low extracluster one.

For a given input vector of $N$ observations, defined as $\mathbf{Y}=\left[\mathbf{y}_{k}\right](k=1, \cdots, N)$, where each $\mathbf{y}_{k}$ is characterized by $P$ features, the membership function can be expressed using a $c \times N$ real matrix $\mathbf{U}=\left[u_{i k}\right]$. The $i^{\text {th }}$ cluster center is then identified by a $P$-dimensional tie-point vector $\mathbf{v}_{i}$.

If cluster centers are not known by a priori considerations, an optimization method has to be applied in order to estimate them. Their locations are iteratively determined by optimizing the following objective function:

$$
J=\sum_{i=1}^{c} \sum_{k=1}^{N}\left(u_{i k}\right)^{m}\left\|\mathbf{y}_{k}-\mathbf{v}_{i}\right\|^{2},
$$

where $d_{i k}^{2}=\left\|\mathbf{y}_{k}-\mathbf{v}_{i}\right\|^{2}$ is the squared Euclidean distance from point $\mathbf{y}_{k}$ to the cluster center $\mathbf{v}_{i}$. The parameter $m$ controls the fuzziness of the algorithm: $m=1$ produces hard partitions of $\mathbf{Y}$, while increasing $m$ allows the single clusters to overlap, blurring the membership degree to higher levels of fuzziness. Since the Euclidean distances of 
the observations from the cluster centers in Equation (6.1) have to be minimized, it is important to scale the different features to the same order of magnitude, in order to avoid having a predominant one, which would affect the classification accuracy. In this work, it has been decided to normalize each input set of features to a unit standard deviation as:

$$
\hat{\mathbf{Y}}_{p}=\mathbf{Y}_{p} / \sigma_{p}
$$

where $\mathbf{Y}_{p}$ is a vector containing the $N$ input values of $\mathbf{Y}$ for the $p^{\text {th }}$ feature and $\sigma_{p}$ is the standard deviation.

By substituting the normalized input data set $\hat{\mathbf{Y}}=\left[\hat{\mathbf{y}}_{k}\right]$ into (6.1), the optimal clustering of $\hat{\mathbf{Y}}$ is therefore obtained as:

$$
(\hat{\mathbf{U}}, \hat{\mathbf{v}})=\arg \min _{\mathbf{U}, \mathbf{v}}(J) .
$$

$\hat{\mathbf{U}}$ and $\hat{\mathbf{v}}$ can be optimized by iterating over the following equations:

$$
\begin{gathered}
\hat{\mathbf{v}}_{i}=\frac{\sum_{k=1}^{N}\left(\hat{u}_{i k}\right)^{m} \mathbf{y}_{k}}{\sum_{k=1}^{N}\left(\hat{u}_{i k}\right)^{m}}, \quad 1 \leq i \leq c, \\
\hat{u}_{i k}=\left(\sum_{j=1}^{c}\left(\frac{\hat{d}_{i k}}{\hat{d}_{j k}}\right)^{\frac{2}{m-1}}\right)^{-1}, \quad 1 \leq k \leq N, 1 \leq i \leq c .
\end{gathered}
$$

After a random initialization of $\hat{\mathbf{U}},(6.4)$ and (6.5) are iteratively updated until convergence is obtained. A convergence test can be performed by computing the mean square error between $\hat{\mathbf{U}}$ at steps iter and iter +1 .

A recurrent issue of the $c$-means clustering algorithm is to remain stuck in a local minimum, being unable to provide a meaningful set of cluster centers. A proper initialization of the cluster centers is therefore highly recommended, as presented in the next section.

\subsubsection{Algorithm Initialization}

The algorithm initialization represents a crucial step in avoiding local minima. Many investigations have been carried out on finding an effective initialization for the algorithm; in this paper, the initialization is based on the work presented in [131]. The input set of normalized observations per feature $\hat{\mathbf{Y}}_{p}$ is transformed into a positive vector $\tilde{\mathbf{Y}}_{p}$ by:

$$
\tilde{\mathbf{Y}}_{p}=\hat{\mathbf{Y}}_{p}-\min \left(\hat{\mathbf{Y}}_{p}\right) \text {. }
$$

Now, the Euclidean distances of each scaled observation from the origin are evaluated and sorted in increasing order. The corresponding scaled observations are sorted accordingly. Given the desired number of output clusters $c$, the sorted observations are grouped together into $c$ subsequent sub-sets, each of those composed of $N / c$ observations. For each sub-set, a cluster center is then initialized by evaluating, for each feature, the mean value of all available observations. 


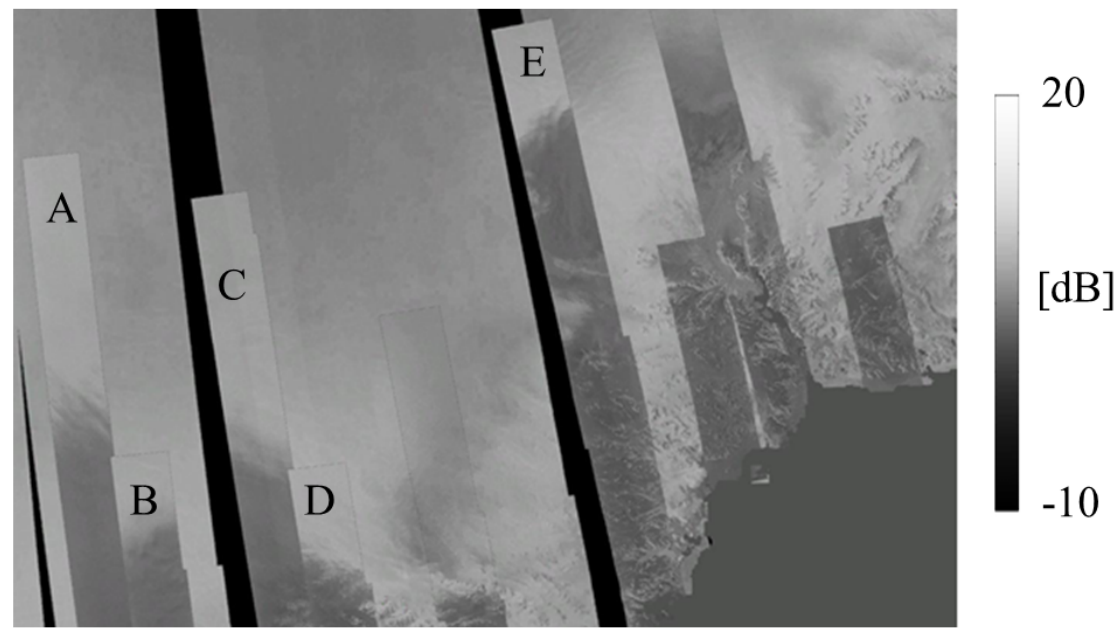

Figure 6.3.: $\gamma^{0}$ over Greenland from TanDEM-X data. Images from summer 2012 (A to E) are superimposed to a mosaic of $\gamma^{0}$, derived from images acquired during winter 2011 only.

\subsection{Input Data: TanDEM-X Mosaics over Greenland}

In this section, the input data set of TanDEM-X interferometric acquisitions, used for classifying Greenland Ice Sheet snow facies, is presented, together with the derivation of a mask of ice/no-ice covered regions to which the current analysis has been confined.

\subsubsection{TanDEM-X Input Mosaics}

The Greenland Ice Sheet has been almost completely covered with TanDEM-X for the first time during winter 2010-2011. All these interferometric SAR acquisitions have been used for the present investigation.

It is worth noting that snow reflectivity changes from winter (typically characterized by dry snow) to summer time (snow melt period). An example is shown in Fig. 6.3, where TanDEM-X $\gamma^{0}$ images from summer 2012 (A to E) are superimposed to a mosaic of $\gamma^{0}$, derived from images acquired during winter 2011 only. Areas affected by snow melt are clearly recognizable by the darker areas and are characterized by the presence of wet snow or even water on the surface, which leads to much lower backscatter levels.

The use of data acquired during winter time only is therefore a necessary condition to assume a reasonable stability of the snow pack, since melt does not significantly occur. The new approach for classifying different snow facies of the Greenland Ice Sheet consists of exploiting the information coming from both the radar backscatter and the volume decorrelation, derived from the interferometric coherence. 

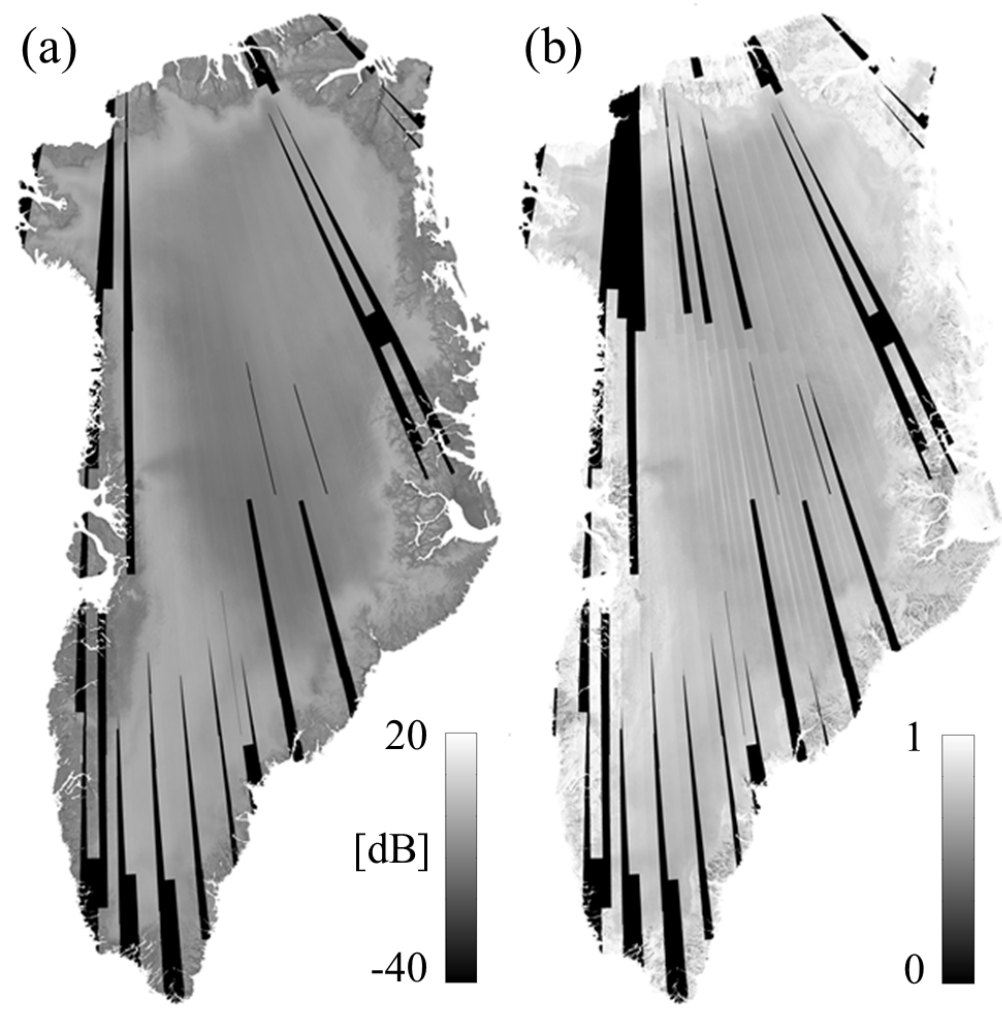

Figure 6.4.: (a) Mosaic of the backscatter $\gamma^{0}$ over Greenland with a resolution of $200 \mathrm{~m}$ $\times 200 \mathrm{~m}$. (b) Corresponding mosaic of the volume correlation factor $\gamma_{\mathrm{Vol}}$. The composed TanDEM-X acquisitions were acquired during winter 2010 2011 only. Areas where no data had been acquired at that time are depicted in black.

Large-scale mosaics of such quantities can be generated by composing quicklook images together, with the constraint of selecting an output resolution equal or larger than the one of each single input image $[83,132]$. For the current work the selected pixel spacing is $0.002^{\circ} \times 0.006^{\circ}$ in latitude/longitude coordinates, which, at a latitude of $73^{\circ} \mathrm{N}$ and longitude of $40^{\circ} \mathrm{W}$, at the center of Greenland, corresponds to a ground resolution of about $200 \mathrm{~m} \times 200 \mathrm{~m}$.

A mosaic of $\gamma^{0}$, the backscatter level projected in the plane perpendicular to the slant range direction, is depicted in Fig. 6.4 (a). This projection has been chosen because, with respect to the other projections in the slant range plane $\left(\beta^{0}\right)$ or on ground $\left(\sigma^{0}\right)$, it is the one which, for an homogeneous type of backscatter, shows a relatively constant reflectivity over a wide range of incidence angles [92]. 
The coherence contribution due to volume decorrelation can be estimated from the total interferometric coherence as explained in section 4.3. The resulting mosaic of $\gamma_{\mathrm{Vol}}$ is shown in Fig. 6.4 (b). It can be seen that, even though the illuminated area on ground is homogeneous, a slight dependency of $\gamma_{\mathrm{Vol}}$ on the slant range for each scene remains, showing a higher $\gamma_{\mathrm{Vol}}$ at the beam's borders (near and far range). This is due to the fact the estimated $\gamma_{\mathrm{SNR}}$ was computed by using theoretical NESZ profiles, caused by a not perfect compensation of the range antenna pattern.

\subsubsection{Generation of the Ice Sheet Mask}

The main part of the Greenland Ice Sheet is flat and is surrounded by mountainous regions characterized by rough topography. Therefore, more gradual variations of both backscatter and topography are expected over the Ice Sheet, with respect to the outer regions. In order to discriminate the Ice Sheet from ice-free areas, a mask is generated by setting thresholds on the local variance of both backscatter and local terrain slope. Single quicklook images of the local slope $\Lambda(x, y)$ have been derived as presented in section 4.3. Moreover, for each $\gamma^{0}$ pixel (in $\mathrm{dB}$ unit), its local variance $\sigma_{\gamma^{0}}^{2}$ has been evaluated as:

$$
\sigma_{\gamma^{0}}^{2}=\mathrm{E}\left[\left(\gamma^{0}\right)^{2}\right]-\left(\mathrm{E}\left[\gamma^{0}\right]\right)^{2}
$$

by computing the mean value $\mathrm{E}[\cdot]$ on a window of $5 \times 5$ pixels around the center one. The local variance of the slope map $\sigma_{\Lambda}^{2}$ has been evaluated following the same approach. Two thresholds have been empirically set at $\sigma_{\gamma^{0}}^{2}=1 \mathrm{~dB}$ and $\sigma_{\Lambda}^{2}=2 \%$. The slope map of Greenland and the corresponding permanent ice mask are depicted in Fig. 6.5 (a) and (b), respectively. Note that the border samples of missing acquisitions are filtered out as well, since the local variance between real data and missing ones within the $\gamma^{0}$ mosaic is obviously high (see Fig. 6.4 (a)).

The obtained Ice Sheet mask has been verified by comparing it to the PROMICE (Programme for Monitoring of the Greenland Ice Sheet) aerophotogrammetric map of Greenland ice masses over different test sites [133]. An example is presented in Fig. 6.6, where the two test sites identified by the red rectangles in Fig. 6.5 (b) are considered. Good results are obtained over the Ice Sheet, while the method frequently fails on outlet glaciers due to the presence of crevasses, small scale features, and steep topography. Nevertheless, the attention is focused on the Ice Sheet as a whole and not on its borders, the derived Ice Sheet mask is assumed to be accurate enough for the purposes of this work. The areas classified to be permanent ice- and snow-covered regions within the TanDEM-X mask are finally taken into account as input observations for locating the different snow facies of the Greenland Ice Sheet. 


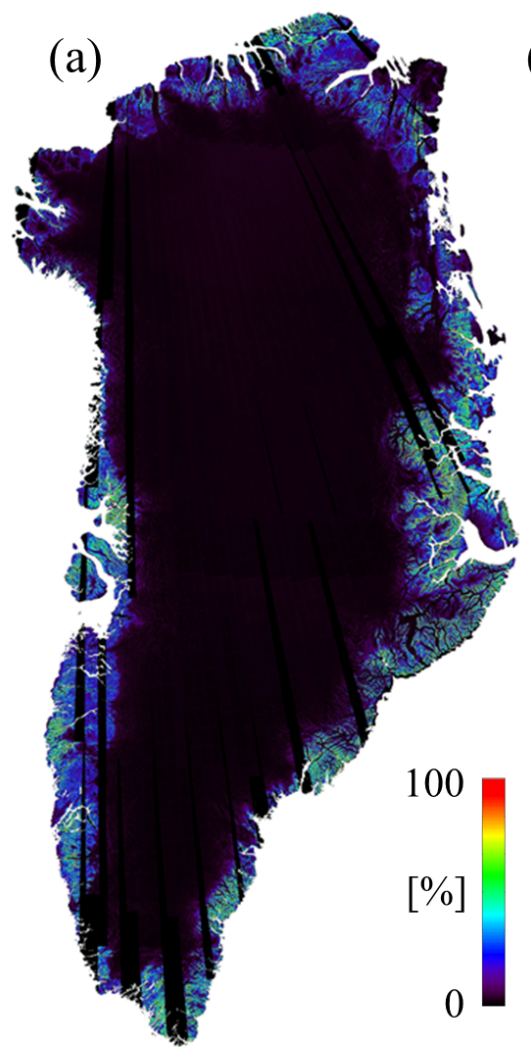

(b)

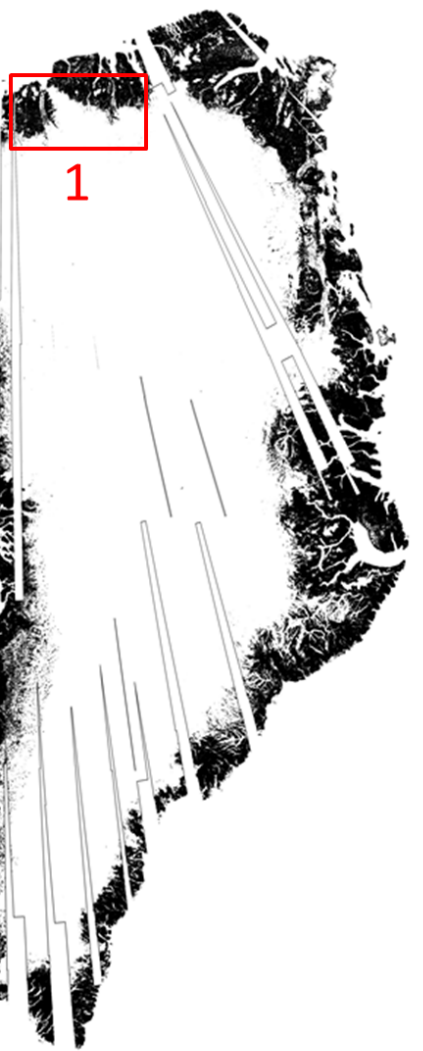

Figure 6.5.: (a) Slope map over Greenland derived from TanDEM-X digital elevation data. (b) Mask of permanent ice areas. White corresponds to the Ice Sheet and black to ice-free areas, derived from the local variance of TanDEM-X backscatter and terrain slope. The red squares identify two test sites used for verification, as presented in Fig. 6.6.

\subsection{Classification Results}

In this section the classification results are presented, obtained by applying the classification method, described in Section 6.2, to the input data set of interferometric TanDEM$\mathrm{X}$ acquisitions, presented in Section 6.3. $P=2$ features have been selected, namely $\gamma^{0}$ and $\gamma_{\text {Vol }}$, and different numbers of clusters. The results obtained using $c=3,4,5$ number of clusters are reported here. The $m$ parameter was set to 2 . The resulting membership maps are displayed in Fig. 6.7. A high percentage corresponds to a high probability of belonging to a specific cluster. The classification results for the three different sets of clusters are presented in Fig. 6.8 (a)-(c). The corresponding normalized histograms of the input data, together with the location of the cluster centers $\hat{\mathbf{v}}$, are depicted in Fig. 6.8 (d)-(f), where the horizontal and vertical axis display the normalized volume correlation 


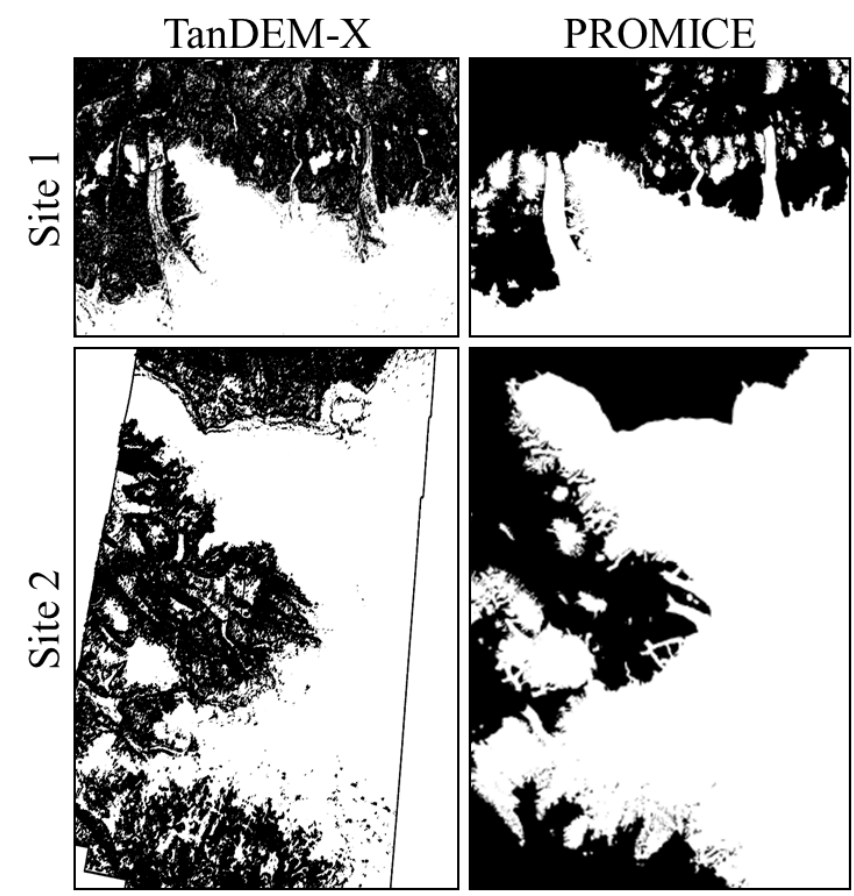

Figure 6.6.: Comparison between masks of permanent ice- and snow-covered regions of Greenland, derived from TanDEM-X interferometric data, and the PROMICE aerophotogrammetric map of Greenland ice masses [133].

factor $\tilde{\gamma}_{\text {Vol }}$ and the normalized backscatter $\tilde{\gamma}^{0}$, evaluated as:

$$
\begin{gathered}
\tilde{\gamma}_{\text {Vol }}=\frac{\gamma_{\text {Vol }}}{\sigma_{\gamma_{\text {Vol }}}}-\min \left(\frac{\gamma_{\text {Vol }}}{\sigma_{\gamma_{\text {Vol }}}}\right), \\
\tilde{\gamma}^{0}=\frac{\gamma^{0}}{\sigma_{\gamma^{0}}}-\min \left(\frac{\gamma^{0}}{\sigma_{\gamma^{0}}}\right),
\end{gathered}
$$

being $\sigma_{\gamma_{\mathrm{Vol}}}$ and $\sigma_{\gamma^{0}}$ the standard deviations of $\gamma_{\mathrm{Vol}}$ and $\gamma^{0}$, respectively. For the considered input data set, one obtains $\min \left(\gamma_{\mathrm{Vol}}\right)=0.14, \sigma_{\gamma_{\mathrm{Vol}}}=0.08, \min \left(\gamma^{0}\right)=-24.7$ $\mathrm{dB}$, and $\sigma_{\gamma^{0}}=3.94 \mathrm{~dB}$. The cluster centers for the selected partition in the normalized histogram of the input data are given in Fig. 6.9.

The fuzzy partition of three clusters shows a higher distance among the single cluster centers. Higher numbers of clusters are also characterized by a higher degree of intercluster overlap and result in a classification where lower values of the membership matrix $\hat{\mathbf{U}}$ are accepted for associating a certain cluster to an input observation. Nevertheless, increasing the number of clusters allows to get a more detailed characterization of the different snow facies, strongly influenced by increasing melt phenomena from the center of the plateau toward the outer edges. The algorithm was run using a higher a-priori 
(a)

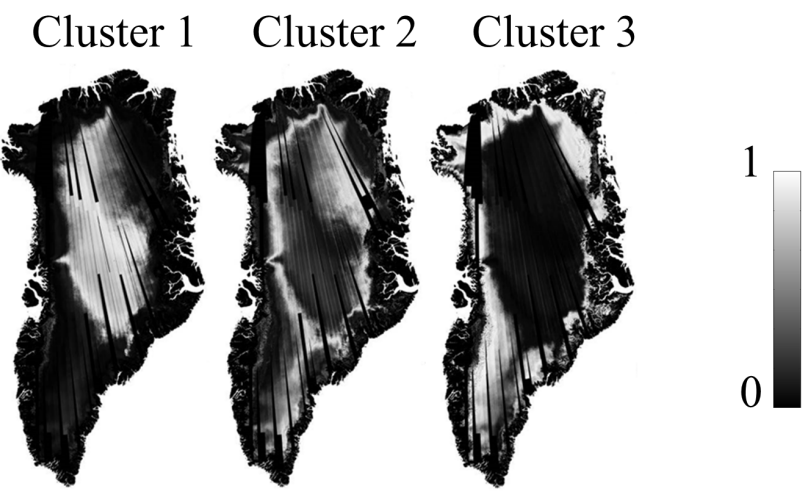

(b)

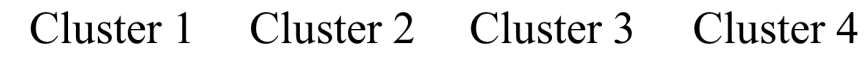

(c)
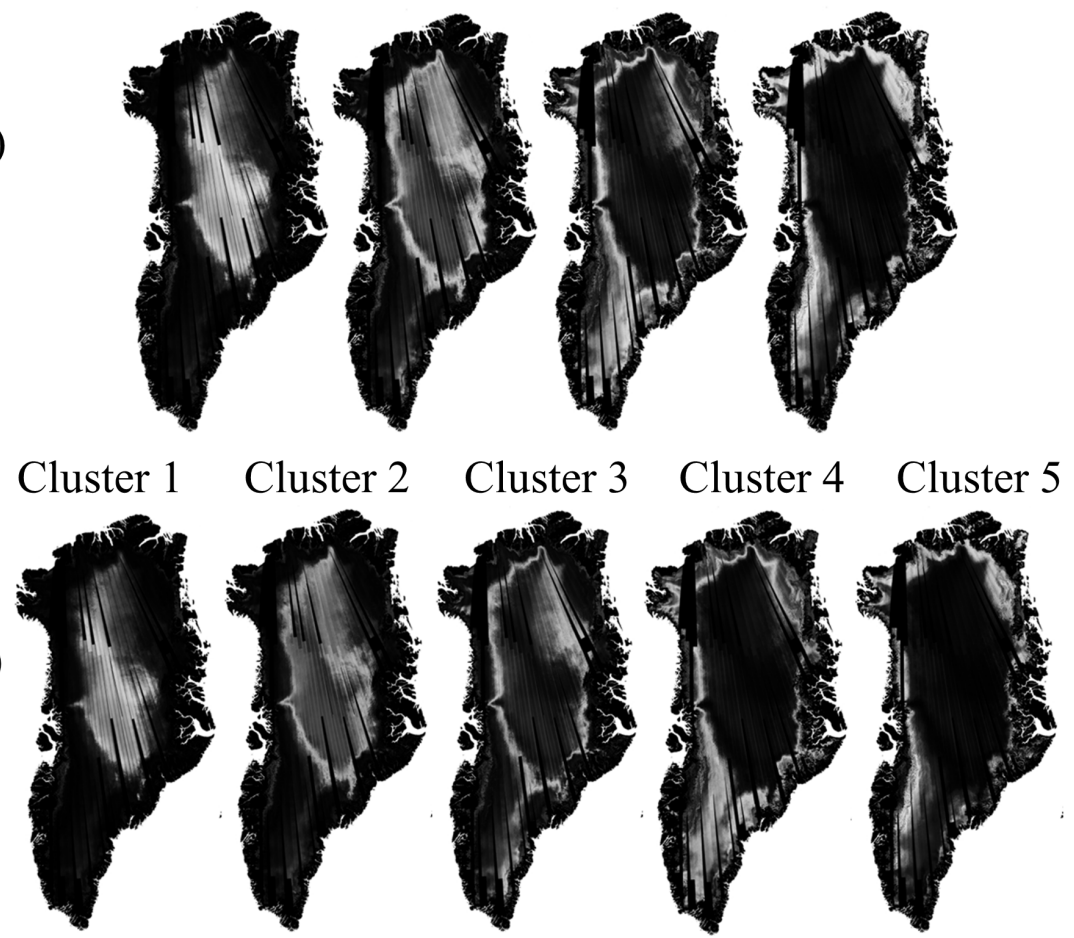

Figure 6.7.: Membership values for each pixel belonging to the Ice Sheet, for the different set of cluster centers used for classifying the Greenland Ice Sheet $(0$ and 1 correspond, respectively, to a probability of $0 \%$ and $100 \%$ of belonging to the considered cluster. Partitions with (a) three, (b) four, and (c) five clusters.

number of clusters $c$ as well, obtaining partitions characterized by a very limited extend and increasing the confusion between adjacent classes. Such a trend is already visible when using $c=5$ (Fig. 6.8 (c)), where cluster 2 (light blue) corresponds to a very thin intermediate layer between cluster 1 (blue) and cluster 3 (green) and is entirely 


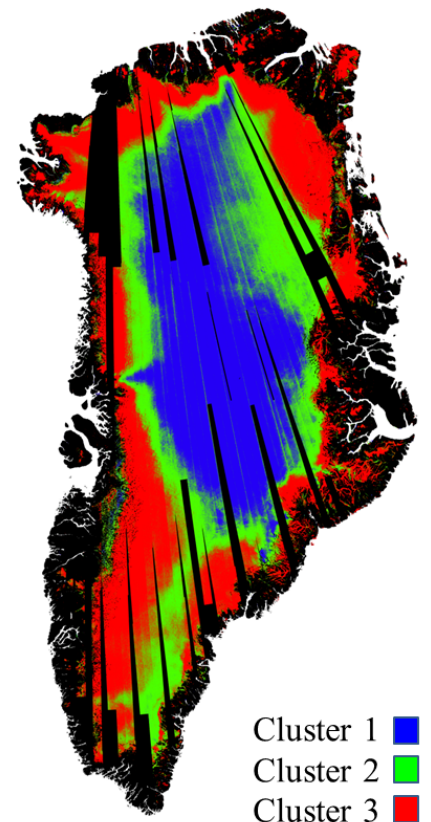

(a)

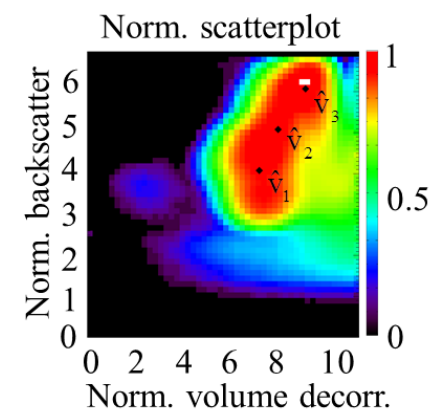

(d)

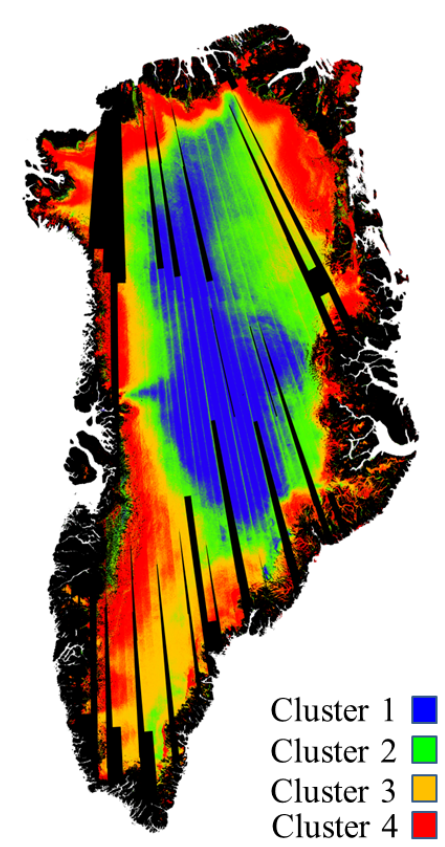

(b)

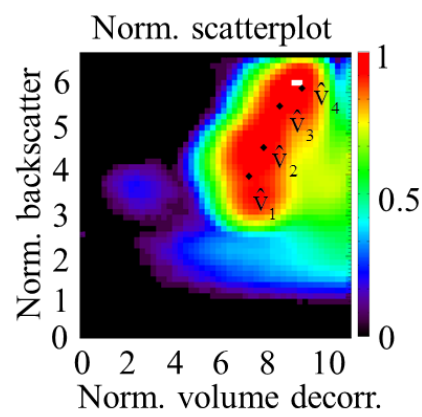

(e)

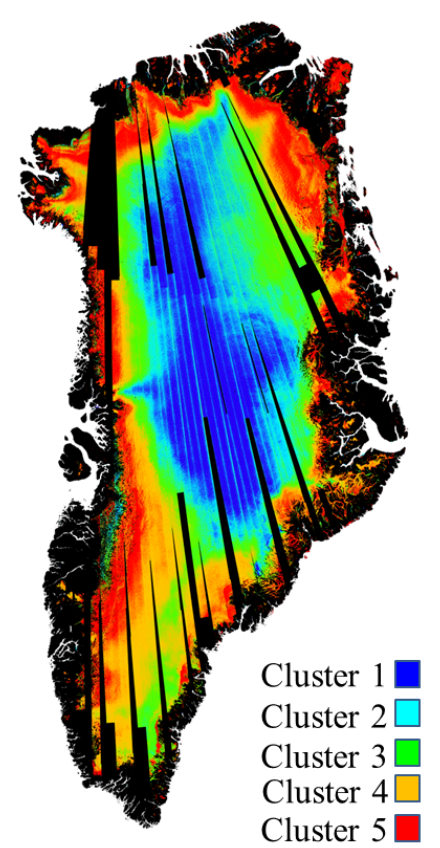

(c)

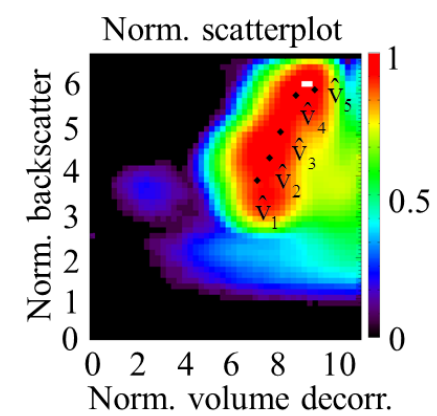

(f)

Figure 6.8.: Classification of the Greenland Ice Sheet facies using (a) three, (b) four, and (c) five clusters; together with the corresponding normalized histograms of the input data and the locations of the cluster centers $\hat{v}((d)-(f))$. The white rectangles locate the maximum of the histogram.

characterized by the presence of pixels classified as both cluster 1 and 3 . This trend is maintained for higher number of cluster centers and the results are here omitted.

Furthermore, for the three different numbers of selected clusters presented in Fig. 6.8, the percentage of pixels classified accordingly to a membership value which is higher than $0.3,0.5,0.7$ and 0.9 are summarized in Tab. 6.1. The results indicate that, using four clusters, over $81 \%$ of the pixels are classified with a membership value above 0.5 . 
Table 6.1.: Percentage of pixels for each set of clusters classified according to a membership value $\hat{u}_{i k}$ higher than $0.9,0.7,0.5$, and 0.3 .

\begin{tabular}{ccccc}
\hline \hline Clusters & $\hat{\boldsymbol{u}}_{\boldsymbol{i k}}>\mathbf{0 . 9 [ \% ]}$ & $\hat{\boldsymbol{u}}_{\boldsymbol{i k}}>\mathbf{P . 7 [ \% ]}$ & $\hat{\boldsymbol{u}}_{\boldsymbol{i k}}>\mathbf{0 . 5 [ \% ]}$ & $\hat{\boldsymbol{u}}_{\boldsymbol{i k}}>\mathbf{0 . 3 [ \% ]}$ \\
\hline 3 & 23.8 & 64.2 & 91.3 & 100.0 \\
\hline 4 & 12.7 & 46.8 & 81.2 & 98.9 \\
\hline 5 & 8.9 & 35.4 & 69.0 & 96.8 \\
\hline
\end{tabular}

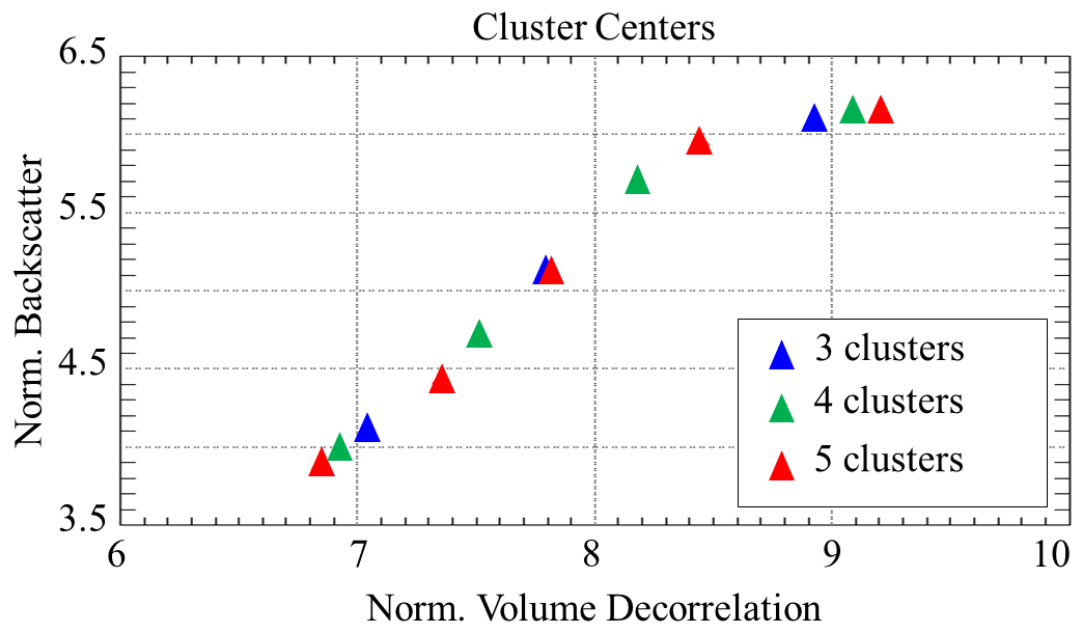

Figure 6.9.: Location of cluster centers in the normalized input domain.

From a pure algorithmic point of view, such a partition shows therefore a reasonably good performance in terms of classification reliability.

Based on this finding, it has been decided to consider the partition with $c=4$ for our further investigation, which represents a good trade-off between a satisfying level of detail and a good separation between adjacent clusters. From now on, the nomenclature will refer to snow facies instead of clusters and the map presented in Fig. 6.8 (b) will be considered as reference, characterized by the presence of 4 different snow facies.

Finally, by considering an overall Ice Sheet surface of $1,700,000 \mathrm{~km}^{2}$, it is possible to estimate the extension of each snow facies. The results are presented in Tab. 6.2. 
Table 6.2.: Extension of the four Ice Sheet snow facies derived with TanDEM-X data, with respect to the overall Ice Sheet surface of $1,700,000 \mathrm{~km}^{2}$, in $\%$ and in $\mathrm{km}^{2}$.

\begin{tabular}{ccc}
\hline \hline Facies & Ice Sheet Percentage [\%] & Extension $\left[\mathrm{km}^{\mathbf{2}}\right.$ ] \\
\hline Facies 1 & 24.1 & 409,700 \\
\hline Facies 2 & 27.8 & 472,600 \\
\hline Facies 3 & 21.9 & 372,300 \\
\hline Facies 4 & 26.2 & 445,400 \\
\hline
\end{tabular}

\subsection{Snow Facies Interpretation and Further Considerations}

The interpretation of the derived snow facies map represents a challenging task, since no global reference data, derived from interferometric SAR acquisitions, is available for a direct comparison. To better understand the properties of each derived snow facies, reference melt data, derived from passive microwave sensors, and in situ measurements (sections 6.5.1 and 6.5.2), was used. This approach allows to characterize the derived snow facies by comparing them to physical parameters such as average amount of snow melt per year, snow density, and structure. Further considerations on the inner snow facies, characterizing statistical parameters, and volume decorrelation dependency on the height of ambiguity are then addressed in sections 6.5.3 and 6.5.5.

\subsubsection{Reference Snow Melt Data}

As reference melt data, the map presented in Fig. 6.10 (b), derived from spaceborne passive microwave sensors as presented by M. Tedesco in [134], was considered. It shows the average amount of melt days per year during the time span between 1981 and 2010 , at a resolution of $25 \mathrm{~km} \times 25 \mathrm{~km}$. Such a long time span has been taken into account in order to be able to identify the dry snow zone, where no significant melt has occurred for at least several years before the observations were carried out.

It is well know that a melt anomaly occurred in summer 2012, characterized by the presence of melt events across almost the entire Ice Sheet. This caused melt/freeze metamorphism with a consequent increase of backscatter levels also over the Greenland dry snow zone [135]. This fact resulted in reduced penetration for CryoSat [136] and possibly also for TanDEM-X. It is therefore important to notice that the data used for the current investigation was acquired before summer 2012, after a quite stable period of several years, documented by the collection of data provided by the C-band scatterometer ASCAT from 2007 onwards [137].

To perform the comparison, the borders between different snow facies of the TanDEM- 


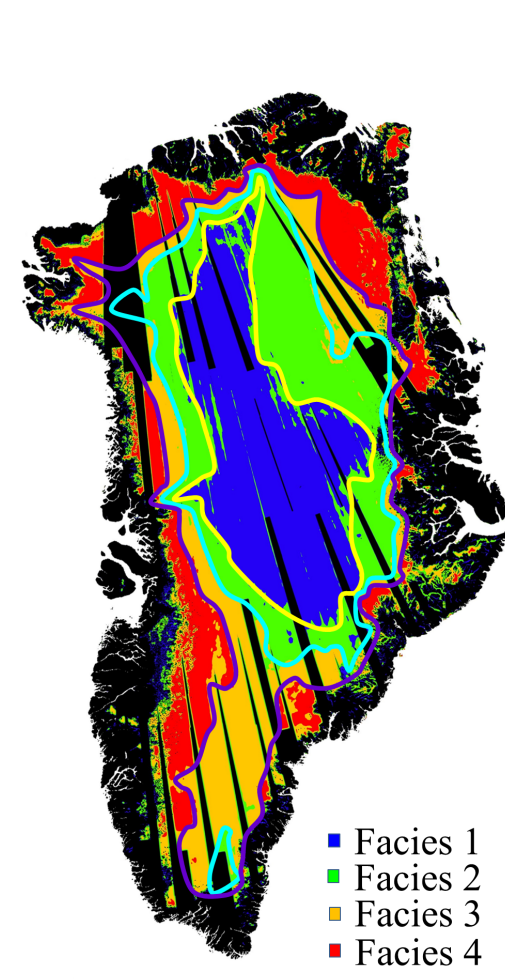

(a)

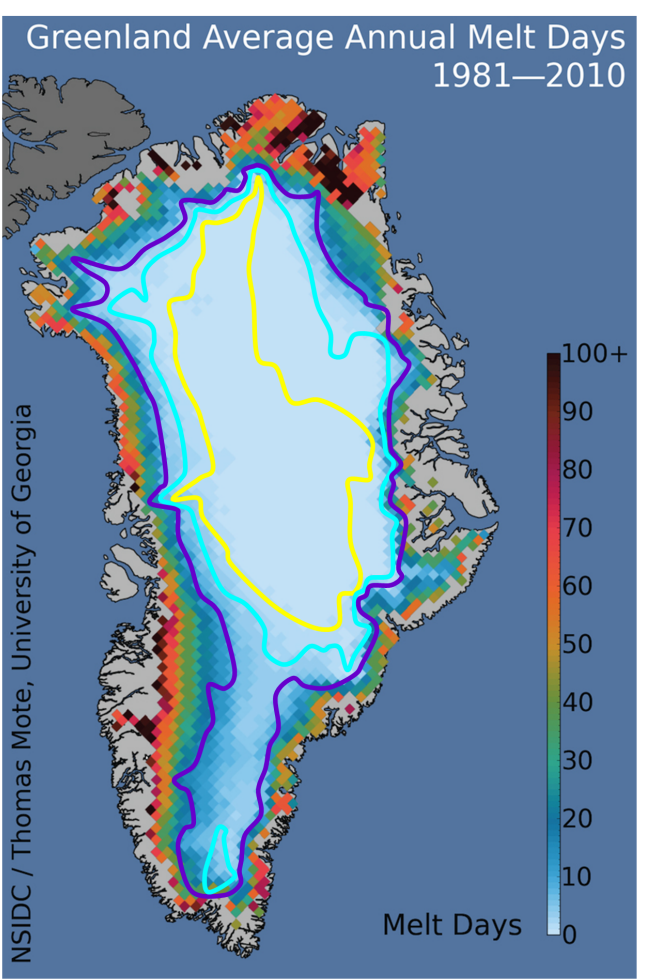

(b)

Figure 6.10.: (a) Estimated snow facies of the Greenland Ice Sheet from TanDEM-X data with a ground resolution of about $4 \mathrm{~km} \times 4 \mathrm{~km}$. The contour lines identify the borders between facies 1 and 2 (yellow), facies 2 and 3 (light blue), and facies 3 and 4 (violet), respectively. (b) Average melt days per year from 1981 to 2010, derived from passive microwave sensors [134].

X classification map are derived. To do so, the classification map in Fig. 6.8 (b) was firstly low-pass filtered using a two-dimensional Gaussian low-pass filter with passband bandwidth at $-3 \mathrm{~dB}$ equal to $5 \%$ of the total bandwidth, obtaining a map with resolution of about $4 \mathrm{~km} \times 4 \mathrm{~km}$ on ground, and then re-quantized it to the 4 available cluster values by applying nearest-neighbor interpolation. The obtained results are shown in Fig. 6.10 (a). At this point, three polygons were manually derived by visual inspection, by selecting tie-points at the border between two adjacent classes (Fig. 6.10 (a)): between the snow facies 1 and 2 (yellow), 2 and 3 (light blue), and 3 and 4 (violet), respectively. The same polygons have also been superimposed onto the melt data in Fig. 6.10 (b).

Facies 1 is mostly unaffected by snow melt. Facies 2 is confined between only a few days and less than ten days of melt per year, while facies 3 is located where melt starts to 


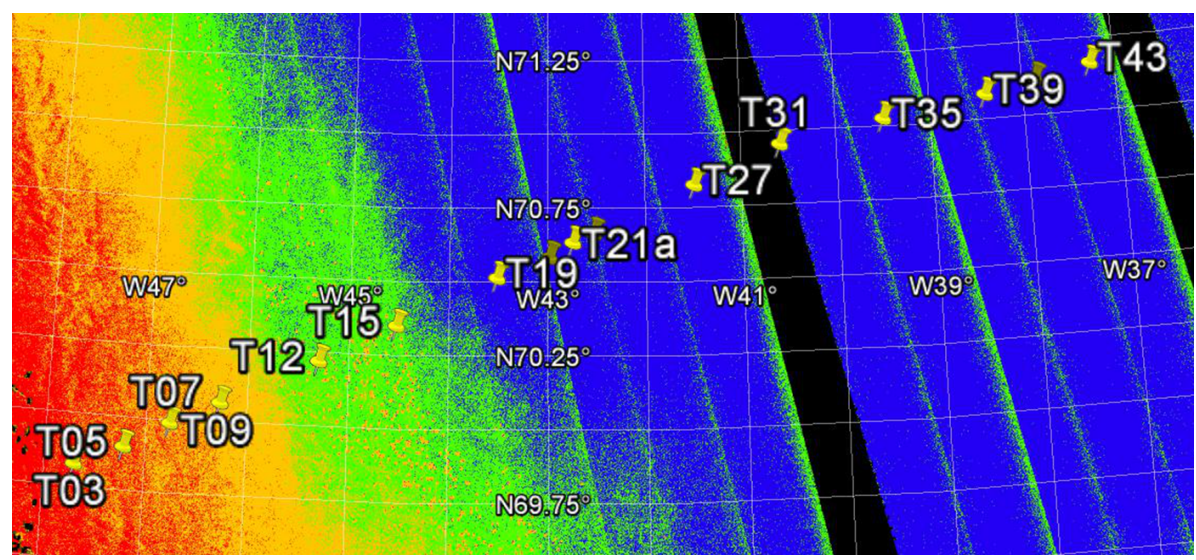

Figure 6.11.: Test sites along the EGIG line used for supporting the CryoSat mission with in situ measurements, superimposed on the snow facies map as derived in Fig. 6.8b (data overlayed in Google Earth).

be considerable (between about ten and twenty days per year). Finally, facies 4 is mostly characterized by more than 20 days of melt per year. The first consideration that can be drawn from this analysis is that facies 1 is principally characterized by the presence of permanently dry snow while the other snow facies belong to a transition zone with increasing melt phenomena toward the outer regions of the Ice Sheet.

\subsubsection{In Situ Measurements along the EGIG Line}

Up to now, many scientific expeditions have been carried out in Greenland to empirically collect data on the state of the Ice Sheet. In particular, several of those acquired data along sections of the Expédition Glaciologique Internationale au Groenland (EGIG) line, a traverse route across the plateau at about $70^{\circ} \mathrm{N}$ [138]. In the last years, selected test sites along the EGIG line were used for supporting the CryoSat Mission [139] with in situ measurements. Some of those are presented in Fig. 6.11, where test sites from T03 to T43 are superimposed on the derived snow facies map.

Scott et al. in [140] analyzed the spatial variation of snow density with depth from in situ measurements at T03, T05, T07, and T12, performed in spring and autumn 2004 and spring 2006. They report that:

- T03 (belonging to facies 4) shows the presence of percolation features, such as ice layers and lenses, generated by meltwater and positioned under the summer melt level.

- at T05 (situated at the transition between facies 3 and facies 4), percolation features do not always reach the melt surface of the previous summer. Moreover, 
because of percolation, an additional moderate densification was observed beneath the previous upper end of summer surface, suggesting that most of the percolating water refreezes before reaching the previous summer surface.

- Between T07 and T12 (situated approximately at the outer and inner borders of facies 3 ) the depth at which percolation features could be found significantly decreased.

Morris and Wingham reported snow density measurements from T05 to T41 in [141], obtained using the neutron probe during field campaigns from 2004 to 2006 . From their work, one can assess that:

- T05 is situated in the percolation zone and characterized by the considerable presence of thick ice layers within the snow pack.

- A transition zone has then been detected between T05 and T21, which matches the borders of the dry snow zone.

- T21 (belonging to facies 1) is indicated as the start of the dry snow zone. From our analysis, the assigned snow facies 1 appears in that region to be slightly more extended toward the outer Ice Sheet of about $30 \mathrm{~km}$.

- Significant differences in the vertical structure are detectable between T12 and T21 (Fig. 5 in [141]), being high-density melt layers clearly visible at T12 only.

- Mean snow density, accumulation rate, and mean snow temperature decrease almost gradually along the EGIG line (from outer to inner regions).

These observations suggest that changes in mean snow density are not suitable for explaining the significant differences in the observed signatures of backscatter and volume correlation factor among the estimated facies, since variations of the mean snow density are small. More relevant are the variations in the stratification and micro-structure of firn, such as the variations of density with depth and the presence of percolation features. As presented by Scott et al. in [142], ice inclusions in the percolation zone produce a complex radar return, characterized by the superposition of multiple strong reflectors within a volume, impacting the characteristic interferometric signature of the acquired radar signal. From the distributions of both backscatter and volume correlation factor from each estimated facies, it can be seen how, within the transition zone, outer facies 3 and 4, where a significant presence of percolation features is detected (e.g., at T03 and T05), are characterized by higher values of both backscatter and volume correlation factor. Facies 2 , on the contrary, is characterized by lower values of both quantities, probably related to the decrease of percolation features within the snow pack. 

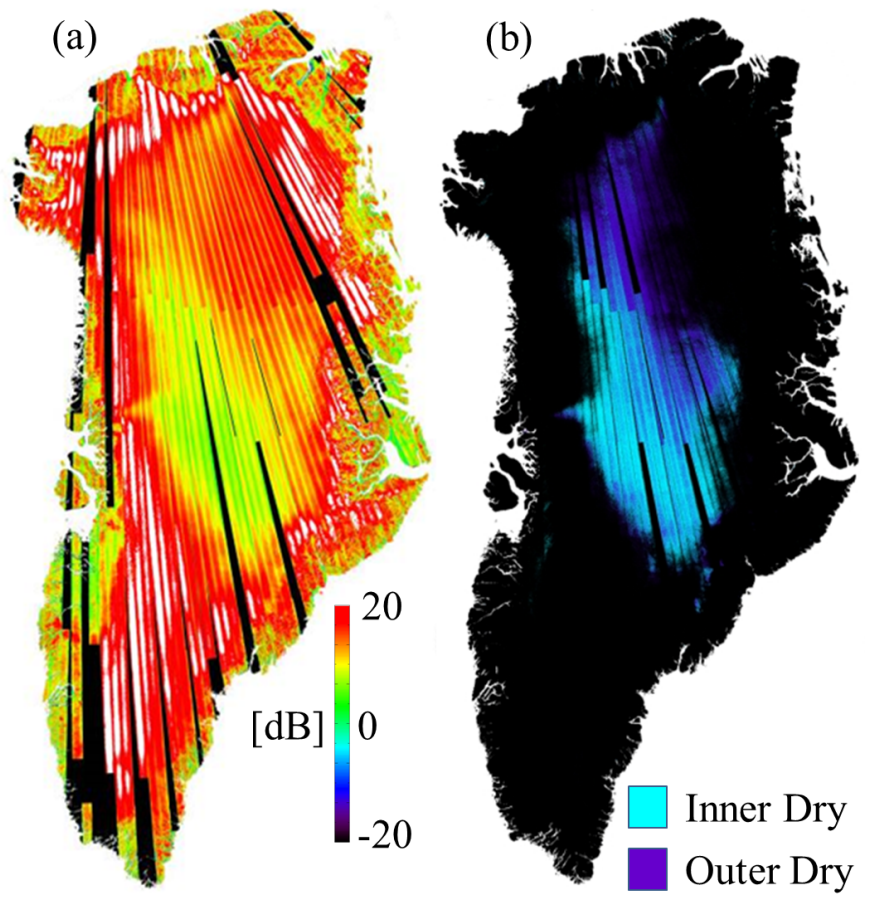

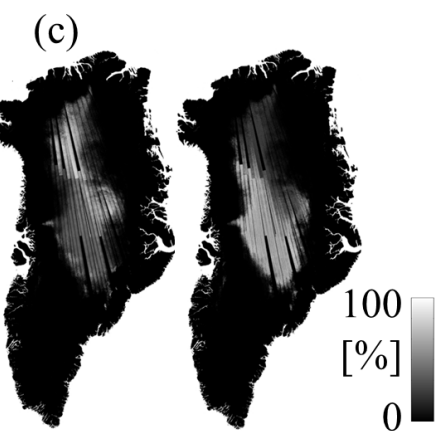

Subcl. 1 Subcl. 2

(d) Norm. Histogram

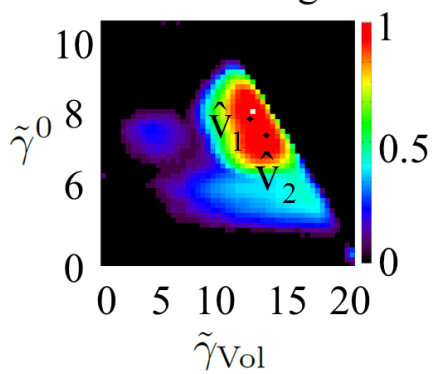

Figure 6.12.: (a) Mosaic of the SNR for the considered TanDEM-X acquisitions. (b) Refined classification of facies 1 into a northern (violet) and a southern (light blue) sub-facies. (c) Membership values for each pixel for the two sub-clusters. (d) Histogram of the input data in the normalized domain.

\subsubsection{Refined Classification of the Inner Snow Facies}

Based on the considerations drawn in Section 6.5.1, one can assume the derived inner snow facies (facies 1) to be dominated by the presence of dry snow. Even if melt does not occur within the whole dry snow zone, this snow facies is characterized by the presence of different types of snow. This can be explained by the lower snow accumulation rate and larger grain size in the north-east part of Greenland [114, 143]. Such differences cannot be so clearly detected by $\gamma_{\mathrm{Vol}}$, but are well visible if $\gamma^{0}$ is considered. This becomes clear when looking at the mosaic of the SNR in Fig. 6.12 (a), which is lower in the inner southern part of snow facies 1 , resulting into a decrease of $\gamma_{\mathrm{SNR}}$. Such loss in the total coherence is therefore compensated with the evaluation of $\gamma_{\mathrm{Vol}}$. A refinement of the classification of facies 1 can be done by performing a sub-clustering, taking into account as input data pixels classified as dry snow zone. In this way, the algorithm is able to detect smaller differences than when applied to the whole Ice Sheet. The results using two sub-clusters are presented in Fig. 6.12 (b), together with the membership matrix 

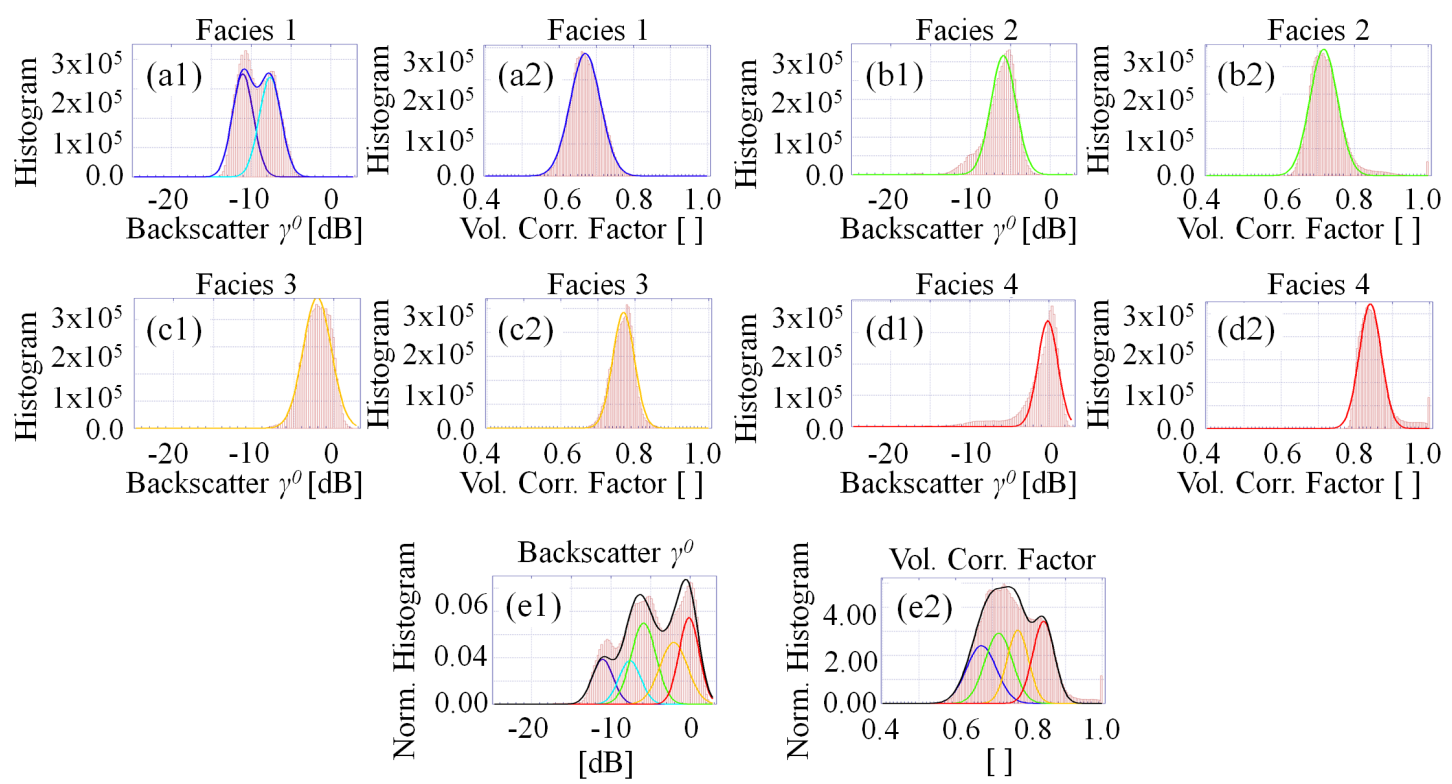

Figure 6.13.: Histograms of the backscatter $\gamma^{0}$ and of the volume correlation factor $\gamma_{\text {Vol }}$ (filled red area), and corresponding Gaussian fitting (solid curves) for facies $1(\mathrm{a} 1, \mathrm{a} 2)$, facies $2(\mathrm{~b} 1, \mathrm{~b} 2)$, facies $3(\mathrm{c} 1, \mathrm{c} 2)$, and facies $4(\mathrm{~d} 1, \mathrm{~d} 2)$. The sum of two Gaussian curves in plot (a1) has been used for fitting the $\gamma^{0}$ of inner snow facies (facies 1). (e1) Overall normalized histogram of the total backscatter $\gamma^{0}$ (red bars) and sum of the five fitting Gaussian distributions (black) derived for the different facies. (e2) Overall normalized histogram of the total volume correlation factor (red bars) and sum of the four fitting Gaussians (black) derived for the different facies.

in Fig. 6.12 (c), and the normalized histogram of the input data in Fig. 6.12 (d). The following values were obtained from the input data for the computation of $\tilde{\gamma}_{\mathrm{Vol}}$ and $\tilde{\gamma}^{0}$ : $\min \left(\gamma_{\mathrm{Vol}}\right)=0.14, \sigma_{\gamma_{\mathrm{Vol}}}=0.04, \min \left(\gamma^{0}\right)=-24.7 \mathrm{~dB}$, and $\sigma_{\gamma^{0}}=2.09 \mathrm{~dB}$. The two classes can be associated to a southern (light blue) and a northern (violet) sub-facies, characterized by higher and lower snow accumulation rates, respectively.

\subsubsection{Statistical Analysis of the Derived Snow Facies}

One can now analyze the statistical properties of the derived snow facies in terms of backscatter $\gamma^{0}$ and volume correlation factor $\gamma_{\mathrm{Vol}}$. To do so, the histograms of these quantities for each snow facies are separately evaluated, as presented in Fig. 6.13 (filled red area). The histograms of backscatter $\gamma^{0}$ have been directly derived from $\gamma^{0}$ in $\mathrm{dB}$. 
Table 6.3.: Mean values and standard deviations of $\gamma^{0}$ and $\gamma_{\mathrm{Vol}}$ for the different snow facies. $\gamma^{0}$ values are displayed in both linear and logarithmic scale.

\begin{tabular}{ccccccc}
\hline \hline & \multicolumn{4}{c}{ Backscatter $\gamma^{\mathbf{0}}$} & \multicolumn{2}{c}{ Volume Correlation Factor $\gamma_{\text {Vol }}$} \\
Facies & \multicolumn{2}{c}{ Mean } & \multicolumn{2}{c}{ Std. Dev. } & Mean & Std. Dev. \\
\hline & {$[$ ] } & {$[\mathbf{d B}]$} & {$[$ ] } & {$[\mathbf{d B}]$} & {[]} & {[]} \\
\hline 1 (southern) & 0.095 & -10.21 & 0.033 & -14.76 & 0.67 & 0.04 \\
1 (northern) & 0.172 & -7.63 & 0.067 & -11.73 & & \\
\hline 2 & 0.253 & -5.96 & 0.101 & -9.95 & 0.73 & 0.05 \\
\hline 3 & 0.664 & -1.78 & 0.247 & -6.07 & 0.77 & 0.03 \\
\hline 4 & 0.883 & -0.54 & 0.365 & -4.37 & 0.85 & 0.04 \\
\hline
\end{tabular}

As it can be seen, each distribution can be clearly fitted by using a Gaussian function, except for $\gamma^{0}$ of facies 1, where two peaks are visible and a better fitting can be performed by summing two Gaussian distributions (Fig. 6.13 (a1)), as confirmed by the refined classification explained in section 6.5.3. The variation of $\gamma_{\mathrm{Vol}}$ from the southern to the northern facies 1 is much less accentuated and a single Gaussian fitting has been used for the whole facies. Mean values and standard deviations, evaluated in linear scale, of $\gamma^{0}$ and $\gamma_{\text {Vol }}$ for the different snow facies are presented in Tab. 6.3.

The fitted Gaussian distributions can now be used to characterize the overall distribution of $\gamma^{0}$ in $\mathrm{dB}$ and $\gamma_{\mathrm{Vol}}$ over the Greenland Ice Sheet as:

$$
G(x)=\sum_{i=1}^{N_{f}} \frac{1}{\sqrt{2 \pi \nu_{1, i}^{2}}} \exp \left(-\frac{\left(x-\nu_{2, i}\right)^{2}}{2 \nu_{3, i}^{2}}\right)
$$

where $N_{f}$ identifies the number of facies and $x$ is either $\gamma^{0}\left(N_{f}=5\right)$ or $\gamma_{\text {Vol }}\left(N_{f}=4\right)$. The estimated set of fitting coefficients $\nu$ is given in Tab. 6.4. The overall normalized histograms of both $\gamma^{0}$ and $\gamma_{\text {Vol }}$, together with the corresponding modeled Gaussian distributions, are shown in Fig. 6.13 (e1,e2).

\subsubsection{Volume Decorrelation Dependency on the Height of Ambiguity}

In presence of volume scattering, the volume correlation factor $\gamma_{\mathrm{Vol}}$ is influenced by the acquisition geometry. The phenomena can be analyzed in terms of dependency of $\gamma_{\text {Vol }}$ on the height of ambiguity $h_{\mathrm{amb}}$, defined as in (3.44). Several investigations on this topic have been already performed within the TanDEM-X mission over forested areas, showing a significant increase of $\gamma_{\text {Vol }}$ with $h_{\mathrm{amb}}$ [144]. 
Table 6.4.: Gaussian fitting coefficients for modeling the distributions of $\gamma^{0}$ and $\gamma_{\text {Vol }}$ as in (6.10).

\begin{tabular}{ccccccc}
\hline \hline \multirow{2}{*}{ Facies } & \multicolumn{3}{c}{ Backscatter $\gamma^{0}$} & \multicolumn{3}{c}{ Volume Correlation Factor $\gamma_{\text {Vol }}$} \\
& $\nu_{1, i}$ & $\nu_{2, i}$ & $\nu_{3, i}$ & $\nu_{1, i}$ & $\nu_{2, i}$ & $\nu_{3, i}$ \\
\hline 1 (southern) & 0.127 & -11.056 & 1.316 & 0.247 & 0.670 & 0.041 \\
1 (northern) & 0.129 & -7.620 & 1.373 & & & \\
\hline 2 & 0.274 & -5.888 & 1.561 & 0.274 & 0.717 & 0.037 \\
\hline 3 & 0.235 & -2.087 & 1.761 & 0.223 & 0.769 & 0.029 \\
\hline 4 & 0.235 & -0.148 & 1.256 & 0.254 & 0.839 & 0.029 \\
\hline
\end{tabular}

Since such a dependency changes with the characteristics of the snow pack and the scattering mechanisms involved, it has not been possible to introduce a correction factor on $\gamma_{\mathrm{Vol}}$ before applying the $c$-means fuzzy clustering algorithm to mitigate such effects, being that the location of the different snow facies is unknown. Hence, a statistical analysis has been carried out afterwards for each estimated snow facies separately, aim to better investigate such a dependency. The different $h_{\mathrm{amb}}$ per input acquisition were grouped together in intervals of $0.5 \mathrm{~m}$ each, evaluating the corresponding distribution of $\gamma_{\mathrm{Vol}}$. For each snow facies, it can be noticed that no significant trend is detectable, probably due to the fact that a limited span of heights of ambiguity is being observed. The clearest trend was observed over facies 3 and is presented in Fig. 6.14. The plot shows the mean values and standard deviations of $\gamma_{\text {Vol }}$ for the different height of ambiguity intervals (vertical axis on the left-hand side), while the number of input measurements in logarithmic scale for each interval of the height of ambiguity is depicted in blue (vertical axis on the right-hand side). Results obtained for $h_{\mathrm{amb}}>53 \mathrm{~m}$ and $h_{\mathrm{amb}}<40 \mathrm{~m}$ are to be considered unreliable, since only a few input measurements were available. An overall slight increase of $\gamma_{\mathrm{Vol}}$ of about 0.05 is visible. Therefore, not introducing a $h_{\mathrm{amb}}$-dependent correction factor on $\gamma_{\mathrm{Vol}}$ does not significantly polarize the estimation of the facies location. Nevertheless, this aspect could be taken into account for a future refinement of the proposed method, by iterating the classification algorithm twice and introducing a different $h_{\mathrm{amb}}$-dependent correction factor for each of the estimated snow facies location at the first step.

\subsection{Applications}

In this section, two application examples of the derived snow facies classification map are presented. In section 6.6.1, the backscatter modeling of the Greenland Ice Sheet is described, while the estimation of the X-band penetration depth is shown in section 6.6.2. 


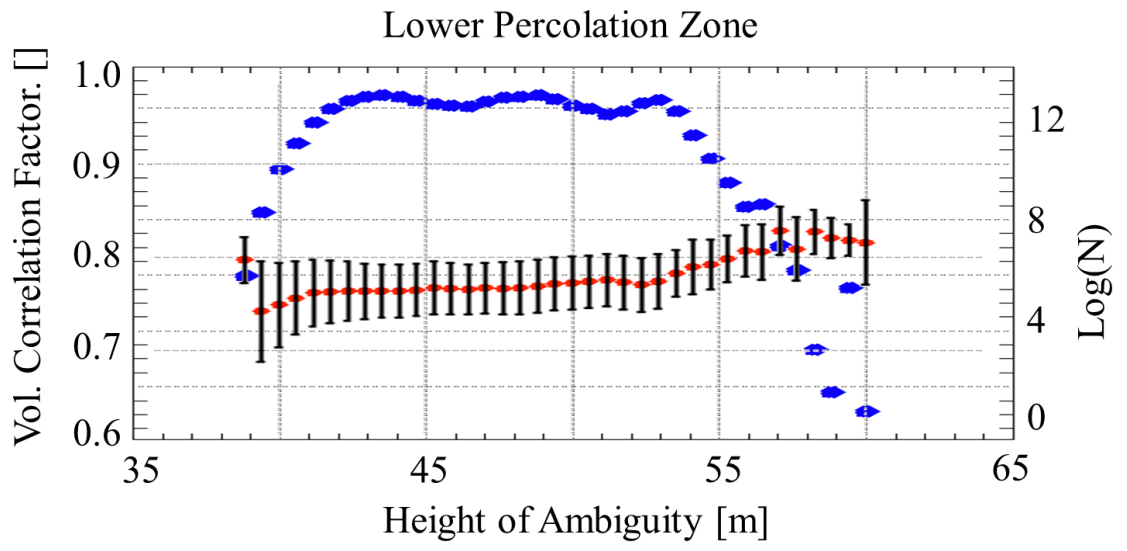

Figure 6.14.: Dependency of the volume correlation factor $\gamma_{\mathrm{Vol}}$ on the height of ambiguity $h_{\mathrm{amb}}$ for the snow facies 3. Red dots: $\gamma_{\mathrm{Vol}}$ mean values, vertical black lines: $\gamma_{\text {Vol }}$ standard deviations (axis on the left-hand side), blue dots: number of available $\gamma_{\text {Vol }}$ samples $N$ per $h_{\text {amb }}$ interval in logarithmic scale (axis on the right-hand side).

\subsubsection{Backscatter Modeling using Modified Weighting}

In this section the backscatter modeling of the Greenland ice sheet is described, based on the classification map derived in the previous sections and on a modified version of the algorithm proposed in chapter 5.

An incidence-dependent backscatter model can now be separately generated for each snow facies derived in section 6.4. Since the characteristics of backscatter are strongly influenced by different snow accumulation rates, a classification map which also includes the refined classification of the dry snow zone, presented in section 6.5.3, has been considered. The complete snow facies classification map is displayed in Fig. 6.15 (a).

Instead of using single acquired scenes separately as in section 5.3.1, the considered input backscatter data is in this case the mosaic of $\gamma^{0}$, presented in section 6.3.1. The Greenland plateau is flat assuring that no significant errors are introduced when deriving $\gamma^{0}$ from $\beta^{0}$. Moreover, in this way, the exact data used for deriving the snow classification map are also used for modeling the backscatter behavior, maximizing the matching between $\gamma^{0}$ values and the corresponding classification. The incidence angles map is depicted in Fig. 6.15 (b).

Instead of using the mean $\gamma^{0}$ derived from a single scene as in section 5.3.1, a proper quality weight has been assigned to each single input $\gamma^{0}$ pixel. The reliability of a single $\gamma^{0}$ value mainly depends on two factor: the reliability of the classification on ground and the corresponding SNR level. 

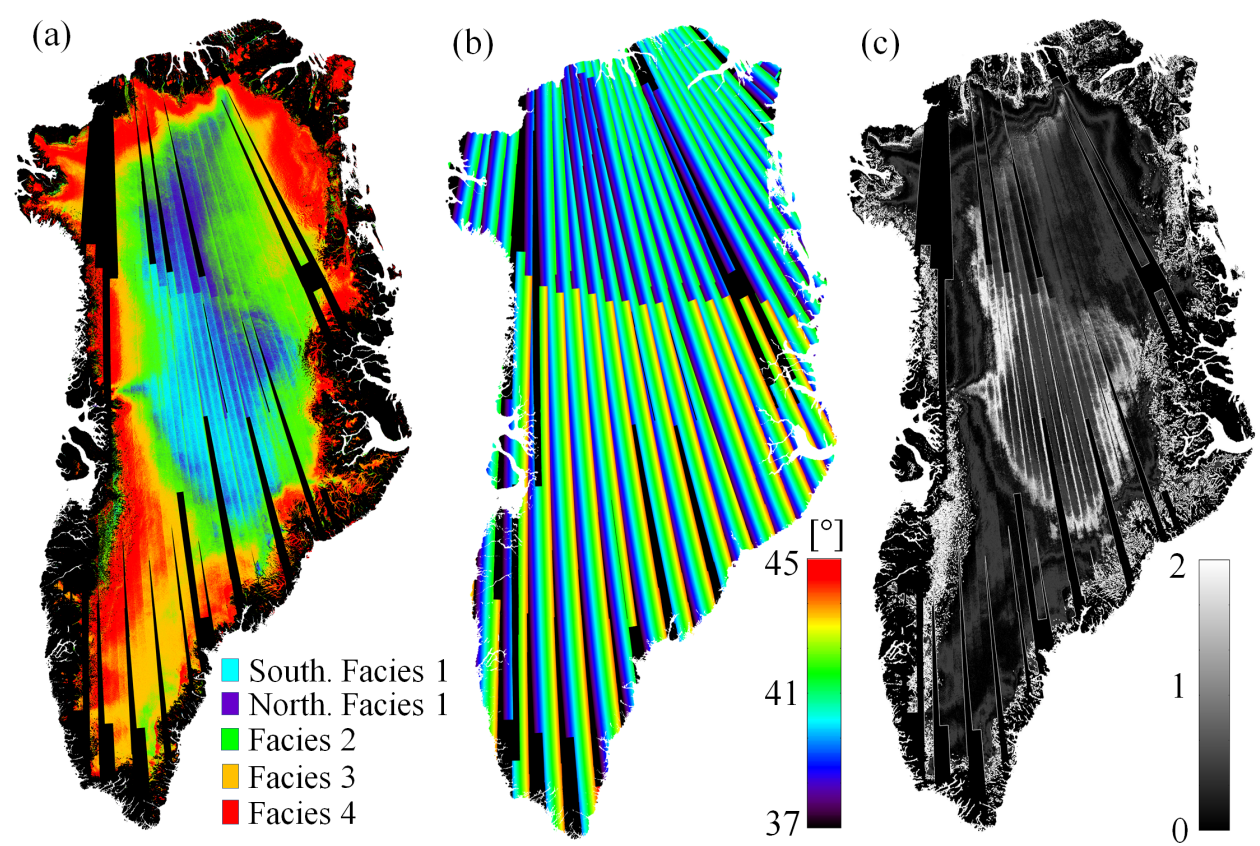

Figure 6.15.: (a) Snow facies map of the Greenland ice sheet, including the dry snow refined classification. (b) Corresponding incidence angles. (c) Local variance of the snow facies map.

The border between different snow facies is significantly blurred, since the properties of snow slowly change from the inside to the coastal regions of the ice sheet. One could therefore decide to quantize the different facies as presented in Fig. 6.10 or, willing to exploit the higher resolution of originally estimated classification map in Fig. 6.8 (b), one can notice that such a classification map is characterized by a higher spatial variation in the intermediate regions between different facies. This can be considered when estimating, for each snow facies, the mean backscatter value per incidence angle interval as presented in (5.16), by introducing quality weights which are inversely proportional to the local variability of the classification map: the more stable the classification map in space, the more reliable a pixel is.

In order to estimate the local variance of the two-dimensional snow facies classification map, the following procedure is proposed. The $N_{\mathrm{s}}$ valid pixels of the snow classification map can now be referred to as $\mathbf{F}=\left[F_{k}\right]$ matrix, with $k=\left[1, \cdots, N_{\mathrm{s}}\right]$, and an integer value is assigned to each class with an increment of one between different classes, which means:

- Southern facies $1 \Rightarrow 1$,

- facies $2 \Rightarrow 2$, 
- facies $3 \Rightarrow 3$,

- facies $4 \Rightarrow 4$,

- Northern facies $1 \Rightarrow 5$.

having therefore $p=5$ different classes.

The local spatial variance $\sigma_{k}^{2 \text { loc }}$ of the $k^{\text {th }}$ pixel of $\mathbf{F}$ can then be evaluated by applying a two-dimensional moving window, namely a boxcar of half width $w_{\mathrm{h}}$, and evaluating the the unbiased sample variance within it as:

$$
\sigma_{j}^{2, \text { loc }}=\frac{1}{N_{\mathrm{w}}-1} \sum_{i=1}^{N_{\mathrm{w}}}\left(F_{i}-\bar{F}_{\mathrm{w}}\right)^{2},
$$

where $\bar{F}_{\text {w }}$ represents the mean value of all $N_{\mathrm{w}}=\left(2 w_{\mathrm{h}}+1\right)^{2}$ pixels within the considered window. For the current analysis a half width $w_{\mathrm{h}}$ of 2 pixels was selected, having a window area of about $1 \mathrm{~km} \times 1 \mathrm{~km}$ on ground. The obtained result is presented in Fig. 6.15 (c).

Each single snow facies can now be separately considered and one can apply a modified version of the algorithm presented in section 5.3.2. For a given snow facies classification, the incidence angles span is divided into $P$ intervals and for the $p^{t h}$ incidence angle interval the following vectors of $N_{p}$ elements can be derived:

- $\gamma^{0}=\left[\gamma_{i}^{0}\right]$ : backscatter $\gamma^{0}$ for each $i^{\text {th }}$ valid pixel from the mosaic in Fig. 6.4 (a) $\left(i=\left[1, \cdots, N_{i}\right]\right)$,

- $\mathrm{SNR}=\left[\mathrm{SNR}_{i}\right]$ : signal-to-noise ratio, as presented in Fig. 6.12 (a),

- $\boldsymbol{\sigma}^{2, \text { loc }}=\left[\sigma_{i}^{2, \text { loc }}\right]$ : local spatial variance as presented in Fig. 6.15 (c).

Quality weights for each input $\gamma^{0}$ pixel are defined by combining SNR and $\boldsymbol{\sigma}^{2 \text {,loc }}$ in the following way. First of all, both quantities are normalized to a unit standard deviation by:

$$
\begin{aligned}
\mathbf{S N} \mathbf{R} & =\mathbf{S N R} / \sigma_{\mathbf{S N R}}, \\
\tilde{\boldsymbol{\sigma}}^{2, \mathrm{loc}} & =\boldsymbol{\sigma}^{2, \mathrm{loc}} / \sigma_{\boldsymbol{\sigma}^{2, \mathrm{loc}}},
\end{aligned}
$$

where $\sigma_{\text {SNR }}$ and $\sigma_{\sigma^{2, \text { loc }}}$ are the standard deviations of SNR and $\boldsymbol{\sigma}^{2, \text { loc }}$ vectors, respectively. In this way, it is assured that there is no predominant contribution between the two of them. The vector of quality weights $\hat{\mathbf{w}}=\left[w_{i}\right]$ for each pixel can then be computed as:

$$
\hat{\mathbf{w}}=\frac{\mathbf{S N} \mathbf{R}}{\tilde{\boldsymbol{\sigma}}^{2, \mathrm{loc}}+\epsilon},
$$




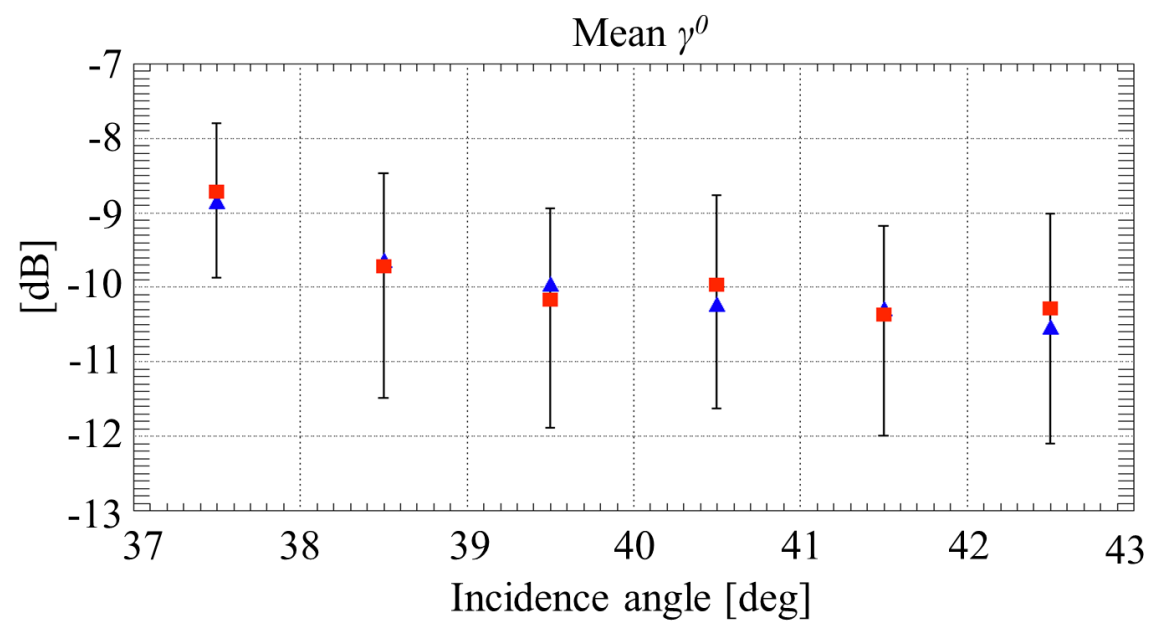

Figure 6.16.: Dry snow zone. Mean $\gamma^{0}$ (triangles), weighted mean $\gamma^{0}$ (squares), and weighted variance (error bars).

where $\epsilon$ is a regularization term to avoid singularities. The weighted mean value $\mu_{p}^{\mathrm{w}}$ and the unbiased weighted variance $\sigma_{p}^{2 \mathrm{w}}$ can now be calculated as:

$$
\begin{aligned}
\mu_{p}^{\mathrm{w}} & =\frac{\sum_{i=1}^{N_{p}} \hat{w}_{i} \gamma_{i}^{0}}{V_{1}} \\
\sigma_{p}^{2 \mathrm{w}} & =\frac{\sum_{i=1}^{N_{\mathrm{j}}} \hat{w}_{i}\left(\gamma_{i}^{0}-\mu_{p}^{\mathrm{w}}\right)^{2}}{V_{1}-\frac{V_{2}}{V_{1}}}
\end{aligned}
$$

where $V_{1}=\sum_{i=1}^{N_{\mathrm{j}}} \hat{w}_{i}$ and $V_{2}=\sum_{i=1}^{N_{\mathrm{j}}} \hat{w}_{i}^{2}$.

The different weighted for each $p^{t h}$ incidence angle interval can finally be grouped together into the following vectors:

$$
\begin{aligned}
\boldsymbol{\mu}^{\mathrm{w}} & =\left[\mu_{1}^{\mathrm{w}}, \cdots, \mu_{P}^{\mathrm{w}}\right], \\
\boldsymbol{\sigma}^{2 \mathrm{w}} & =\left[\sigma_{1}^{2 \mathrm{w}}, \cdots, \sigma_{P}^{2 \mathrm{w}}\right],
\end{aligned}
$$

which are equivalent to the vectors defined in (5.18) and (5.19). An example over the dry snow zone is presented in Fig. 6.16.

The same estimator in (5.24) can now be applied to the current data set to derive the ML polynomial fitting coefficients $\widehat{\boldsymbol{\xi}^{w}}$ and model the $\gamma^{0}$ dependency on the incidence angle as in (5.25), utilizing the estimated weighted variance in the covariance matrix $C_{\mathrm{w}}$. 


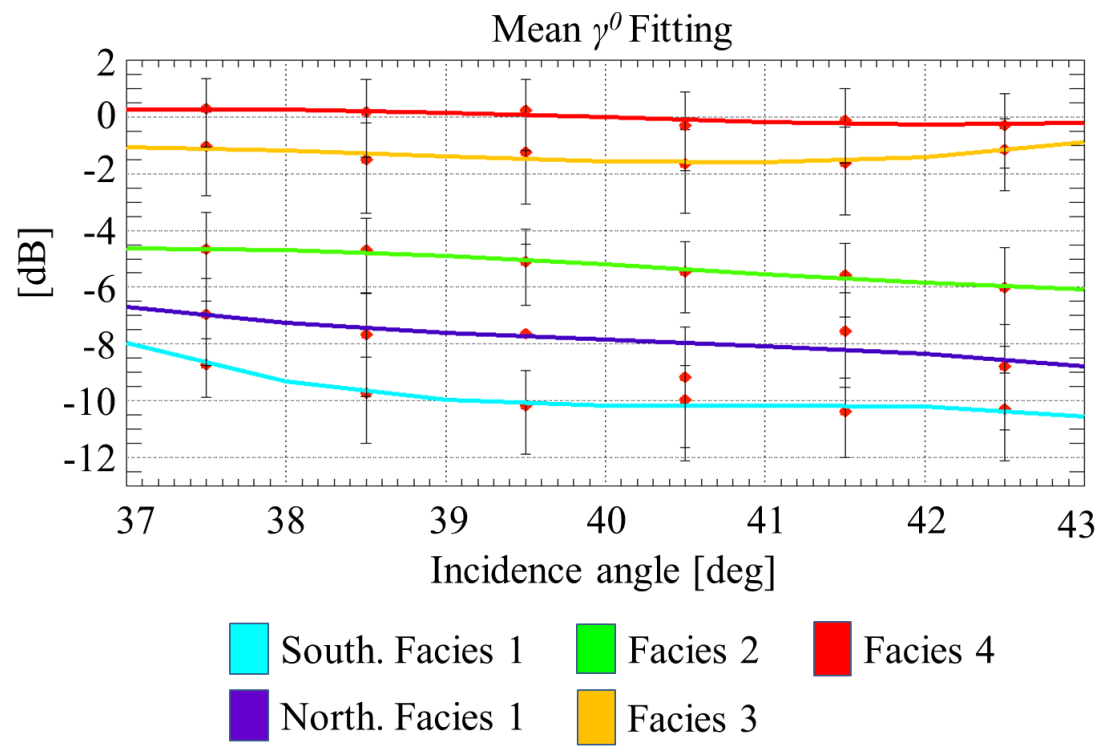

Figure 6.17.: Incidence angle-dependent polynomial backscatter models of $\gamma^{0}$ for the different Greenland snow facies in Fig. 6.15 (a), derived from TanDEM-X winter acquisitions in $\mathrm{HH}$ polarization.

Table 6.5.: Polynomial fitting coefficients of $\gamma^{0}$ for the different snow facies of the Greenland Ice Sheet.

\begin{tabular}{ccccc}
\hline \hline Snow Facies & $\widehat{\xi_{0}^{w}}$ & $\widehat{\xi_{1}^{w}}$ & $\widehat{\xi_{2}^{w}}$ & $\widehat{\widehat{\xi}}$ \\
\hline North facies 1 & 2777.3341 & -204.95934 & 5.0229939 & -0.041029821 \\
\hline South facies 1 & 962.33995 & -71.831205 & 1.7779530 & -0.014713497 \\
\hline Facies 2 & -646.47826 & 48.059520 & -1.1923805 & 0.0097923131 \\
\hline Facies 3 & -950.50485 & 74.042457 & -1.9198327 & 0.016546445 \\
\hline Facies 4 & -646.45231 & 48.985066 & -1.2327992 & 0.010304950 \\
\hline
\end{tabular}

The results for all different snow facies are presented in Fig. 6.17. The derived fitting coefficients are summarized in Tab. 6.5.

\subsubsection{Estimation of the Penetration Depth}

Knowing the properties and the location of the different facies of the Greenland Ice Sheet represents the bases for further scientific investigations. In this section, a map of the penetration depth is derived, based on the model presented by Weber Hoen and Ze- 


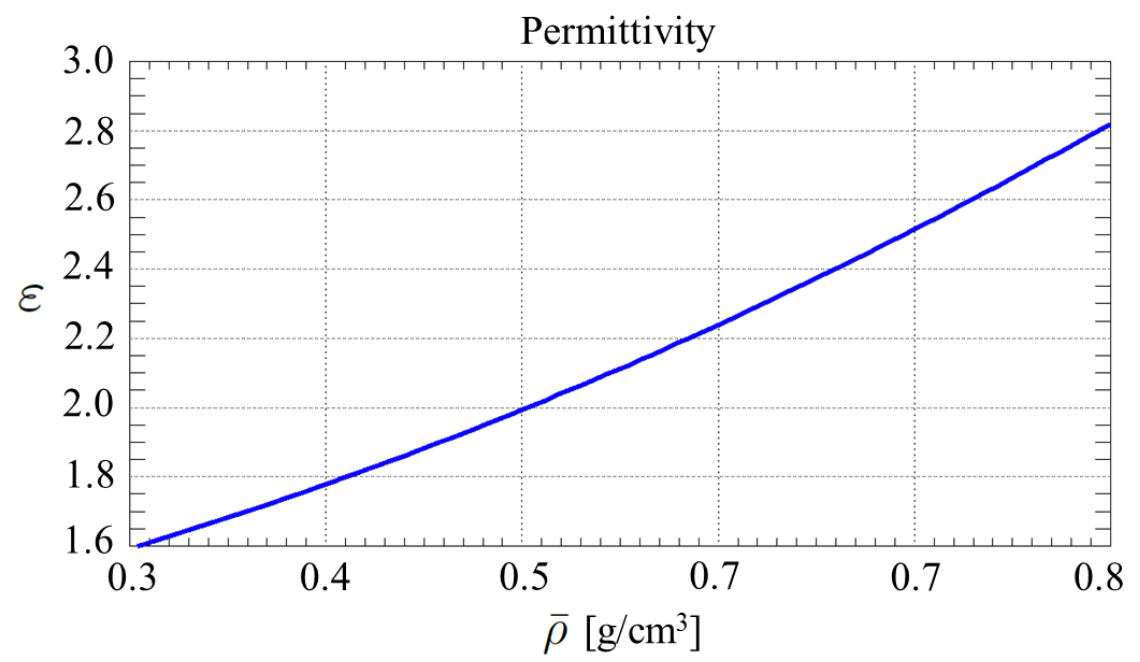

Figure 6.18.: Relation between snow density and permittivity, derived from [145].

bker in [119] and is compared to real elevation measurements from TanDEM-X data. By assuming a homogeneous, lossy scattering medium, they modeled the volume correlation factor $\gamma_{\mathrm{Vol}}$ with respect to the one-way power penetration depth $d_{1 w}$ as:

$$
\gamma_{\mathrm{Vol}}=\frac{1}{\sqrt{1+\left(\frac{2 \pi \sqrt{\varepsilon} d_{1 w} B_{\perp}}{R \lambda \tan \theta}\right)^{2}}}
$$

where $\varepsilon$ is the dielectric constant and, for an icy medium, it is supposed to be real and to remain constant throughout it. $d_{1 w}$ represents the penetration depth where the one-way power decreases by $1 / e$. By inverting (6.19), one obtains $d_{1 w}$ as:

$$
d_{1 w}=\frac{R \lambda \tan \theta}{2 \pi \sqrt{\varepsilon} B_{\perp}} \sqrt{\frac{1}{\gamma_{\text {Vol }}^{2}}-1}
$$

The two-way penetration depth $d_{2 w}$ can then be derived as:

$$
d_{2 w}=\frac{d_{1 w}}{2}
$$

The two-way penetration depth is considered here since it is the one that approximates the location of the radar mean phase center and is therefore related to the measured interferometric height. By exploiting the snow facies map in Fig. 6.10 (a), one can now associate to facies with a proper value of $\varepsilon$. The dielectric constant $\varepsilon$ can be related to the snow density $\rho$ as presented in [145]; taking the single measurements which relate the density to the permittivity in $\mathrm{H}$ polarization, a $2^{\text {nd }}$-order polynomial fitting is performed, 
Table 6.6.: Mean snow density $\bar{\rho}$ and permittivity $\varepsilon$ for the different snow facies of the Greenland Ice Sheet $[141,145]$.

\begin{tabular}{ccc}
\hline \hline Facies & Mean Snow Density $\overline{\boldsymbol{\rho}}\left[\mathbf{g} / \mathbf{c m}^{\mathbf{3}}\right]$ & Permittivity $\boldsymbol{\varepsilon}$ \\
\hline 1 & 0.355 & 1.70 \\
\hline 2 & 0.395 & 1.75 \\
\hline 3 & 0.40 & 1.78 \\
\hline 4 & 0.41 & 1.80 \\
\hline
\end{tabular}

as shown in Fig. 6.18. Assuming a homogenous density of snow within the most superficial layers of the snow pack (until about $10 \mathrm{~m}$ depth), the mean snow density $\bar{\rho}$ along the EGIG line, accumulated over the period of spring 2004 to summer 2006, can be extrapolated from Equation (20) in [141] and associated to the different test sites, depending on the distance from T05 (as in Tab. 1 of [141]), leading to the following values:

- at T05 (belonging to facies 4): $\bar{\rho}=0.41 \mathrm{~g} / \mathrm{cm}^{3}$,

- at T09 (belonging to facies 3): $\bar{\rho}=0.40 \mathrm{~g} / \mathrm{cm}^{3}$,

- at T12 and T15 (belonging to facies 2): $\bar{\rho}=0.40 \mathrm{~g} / \mathrm{cm}^{3}$ and $\bar{\rho}=0.39 \mathrm{~g} / \mathrm{cm}^{3}$,

- from T21 to the summit of the traverse (belonging to facies 1$): \bar{\rho}$ decreases from about $0.38 \mathrm{~g} / \mathrm{cm}^{3}$ to about $0.33 \mathrm{~g} / \mathrm{cm}^{3}$.

When more than a single $\bar{\rho}$ value per facies are available, the mean value has been considered. These values are summarized in Tab. 6.6, together with the corresponding permittivities. By substituting the derived $\varepsilon$ into Equations (6.20) and (6.21), together with the other parameters derived for the considered TanDEM-X acquisitions, we obtain the map of the two-way penetration depth in Fig. 6.19 (a). The corresponding histograms for each facies are depicted in Fig. 6.20 (a) and the mean value E[ $\left.d_{2 w}\right]$ and standard deviation $\sigma_{d_{2 w}}$ for each distribution are summarized in Tab. 6.7.

The obtained results over facies 1 (characterized by dry snow) match very well with the ones obtained by Rott et al. in [120], where a one-way penetration depth of $8.1 \mathrm{~m}$ at $10 \mathrm{GHz}$ was estimated for dry, highly metamorphic snow, corresponding to a two-way penetration depth of $4.05 \mathrm{~m}$.

The obtained results have also been compared to the difference between ICESat laser elevation measurements, carried out between 2003 and 2009 [146], and the final global TanDEM-X DEM. As already mentioned, radar DEMs represent the location of the mean phase center of the backscattered signal; in case of penetration into the snow pack, they 


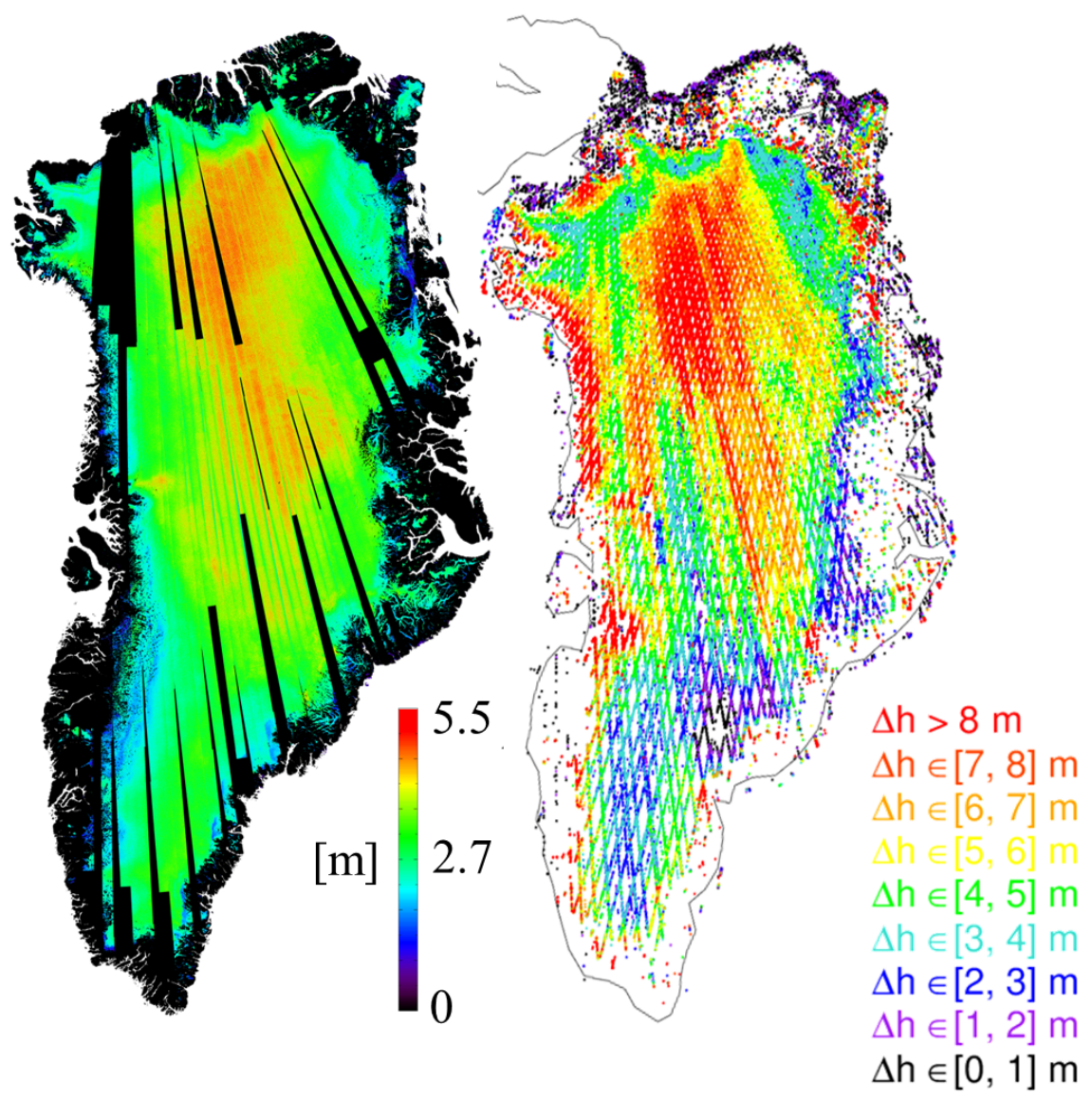

(a)

(b)

Figure 6.19.: (a) Map of the retrieved X-band two-way penetration depth. (b) Mean differences between TanDEM-X DEM, acquired during winter, and ICESat measurements.

will differ from ICESat, which measures the height of the surface. For each available ICESat value, the mean difference $\Delta h$ between ICESat and TanDEM-X DEM is evaluated as:

$$
\Delta h=\mathrm{E}\left[h_{I C E S a t}-h_{T D X}\right]
$$

where $h_{T D X}$ identifies the mean height of the final TanDEM-X DEM within the considered ICESat footprint and $h_{I C E S a t}$ represents the measured height from ICESat over the same ground area. The results are shown in Fig. 6.19 (b). $\Delta h$ has been separately evaluated for the four different snow facies in Fig. 6.10 (a) by applying a defined polygon for each zone, derived as presented in Fig. 6.10. The corresponding histograms for the different facies are depicted in Fig. 6.20 (b) and the mean values and standard deviations are again summarized in Table 6.7. It has to be mentioned that ICESat measurements 

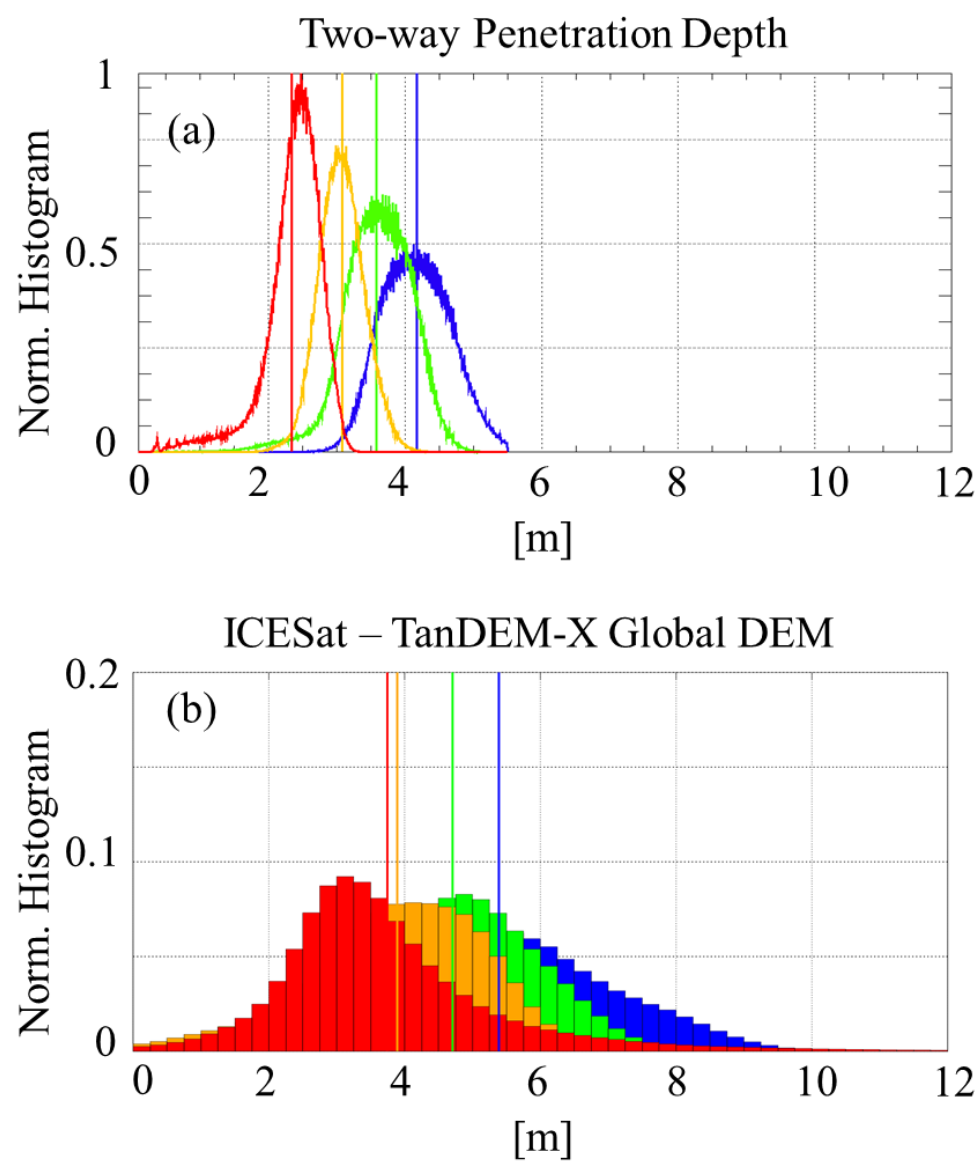

- Facies $1 \quad \square$ Facies 3

$\square$ Facies $2 \square$ Facies 4

Figure 6.20.: (a) Histograms of the two-way penetration depth for the different snow facies. (b) Histograms of the mean difference between ICESat and TanDEM$\mathrm{X}$ DEM $\Delta h$ for the different facies. The mean value of each distribution is indicated by a vertical line in the corresponding color.

are older than the considered TanDEM-X DEMs. In the mean time, the height of the Ice Sheet changed, introducing a further uncertainty in the estimation.

The depth of the mean phase center of a radar wave, measured by the interferometric phase, approximately equals the two-way penetration depth $d_{2 w}$ if the latter is lower than about $10 \%$ of the height of ambiguity $h_{\mathrm{amb}}$, otherwise a bias between the two is introduced [147]. For the current analysis, the worst-case can be estimated using the ratio between the $3 \sigma$ two-way penetration depth over the dry snow zone, given by $\mathrm{E}\left[d_{2 w}\right]+$ 
Table 6.7.: Mean values and standard deviations of the two-way penetration depth and of the difference between ICESat measurements and TanDEM-X (TDX) DEMs for the different snow facies. $\Delta H$ is the difference between the mean $d_{2 w}$ and the mean $\Delta h$.

\begin{tabular}{cccccc}
\hline \hline \multirow{2}{*}{ Facies } & \multicolumn{2}{c}{ Penetration Depth $\boldsymbol{d}_{\mathbf{2} w}$} & \multicolumn{2}{c}{ ICESat-TDX DEM $\boldsymbol{\Delta h}$} & $\boldsymbol{\Delta H}$ \\
& Mean [m] & Std. Dev. [m] & Mean [m] & Std. Dev. [m] & {$[\mathbf{m}]$} \\
\hline 1 & 4.18 & 0.51 & 5.38 & 1.90 & -1.20 \\
\hline 2 & 3.58 & 0.56 & 4.70 & 1.49 & -1.12 \\
\hline 3 & 3.07 & 0.33 & 3.89 & 1.54 & -0.82 \\
\hline 4 & 2.34 & 0.49 & 3.74 & 2.32 & -1.40 \\
\hline
\end{tabular}

$3 \sigma_{d_{2 w}} \simeq 5.5 \mathrm{~m}$, and a minimum $h_{\mathrm{amb}}$ of about $40 \mathrm{~m}$ (Fig. 6.14). The result is a ratio of about $14 \%$, which allows us to reasonably assume that no significant bias is introduced between the two-way penetration depth $d_{2 w}$ and the elevation measurement $\Delta h$. One can now evaluate the difference $\Delta H$ between the mean $d_{2 w}$ and the mean $\Delta h$ for each snow facies as:

$$
\Delta H=\mathrm{E}\left[d_{2 w}\right]-\mathrm{E}[\Delta h] .
$$

Assuming a good accuracy of the two-way penetration depth $d_{2 w}$, at least confirmed for the inner snow facies (characterized by the presence of dry snow) by the results obtained by Rott et al. in [120], $\Delta H$ is expected to be around zero. Even though the results match quite well, the obtained values, shown in Tab. 6.7, indicate the presence of a slightly negative offset which varies from about $-0.8 \mathrm{~m}$ to $-1.4 \mathrm{~m}$. A reason to at least partly explain such differences is the simplified (single layer) model of Hoen and Zebker for relating volume decorrelation to penetration depth. The model assumes that there is no depth dependency of the scattering cross-section, a constant density, and uncorrelated scatterers. This hypothesis is not true for a highly stratified medium such as polar firn, as addressed in section 6.5.2 [140,141]. For example, since the penetration depth at $\mathrm{X}$-band is on the order of a few meters, the density of the upper layers of the snow pack becomes of predominant importance. In particular, the first two meters typically present lower density than the mean values used here (see e.g., Fig. 2 and 5 in [141]). If one now assumes a decrease of $20 \%$ in snow density, which is comparable to the density change in the upper layers in $[140,141]$ with respect to the mean one, this would result in an increase of the mean two-way penetration depth in the range from $7 \mathrm{~cm}$ (facies 1) up to $17 \mathrm{~cm}$ (facies 4), reducing the remaining offsets by the same amount.

Other sources of uncertainty may result from the fact that the TanDEM-X DEM has been calibrated using ICESat measurements in the outer regions of Greenland only. Along the Ice Sheet a self-adjusting block calibration has been implemented [148], which might also explain the persistence of a residual offset. A further reason might be the occur- 
rence of height changes during the time span which separates ICESat measurements from TanDEM-X acquisitions.

A way to improve the accuracy of the penetration depth model could be to combine both backscatter and volume decorrelation information, which would be consistent with the applied snow facies classification method, which considers both quantities. This topic will be the objective of further investigations. 


\section{Generation of Backscatter Maps}

In this chapter, a possible application for a global data set of backscatter models is presented: the generation of backscatter maps, achieved by properly mosaicking an input set of absolutely calibrated $\beta^{0}$ images.

The proposed algorithm can be applied to generate both local- and large-scale backscatter maps, with varying resolutions and equalized to a specific reference incidence angle.

The main content of this chapter was published in [98].

\subsection{Generation of Backscatter Mosaics}

The generation of backscatter mosaic consists of the equalization and combination over a common output grid of a set of absolutely calibrated and geocoded $\beta^{0}$ images. The input data can be stored in a data base, generated as described in section 5.3.1.

The complete procedure flowchart is presented in Fig. 7.1. Firstly, the user has to set a series of input parameters, which include:

- The desired polarization of the output backscatter map,

- The region of interest (ROI), including the desired output pixel spacing,

- The reference incidence angle $\theta_{\text {ref }}$, which corresponds to the incidence angle to which the output backscatter map will be equalized,

- The desired input acquisition time interval.

The $\beta^{0}$ data base is then accessed to retrieve a list of all the available images for the required output map and an interpolator is implemented, in order to convert $\beta^{0}$ values with different incidence angles and land cover types to the reference output incidence angle $\theta_{\text {ref. }}$. The main algorithm steps can be summarized as follows:

1. A ground classification class is associated to each input $\beta^{0}$ pixel that suits the output map requirements (geographic location, acquisition time). For the proposed examples, the ESA GlobCover ground cover classification map is considered as 


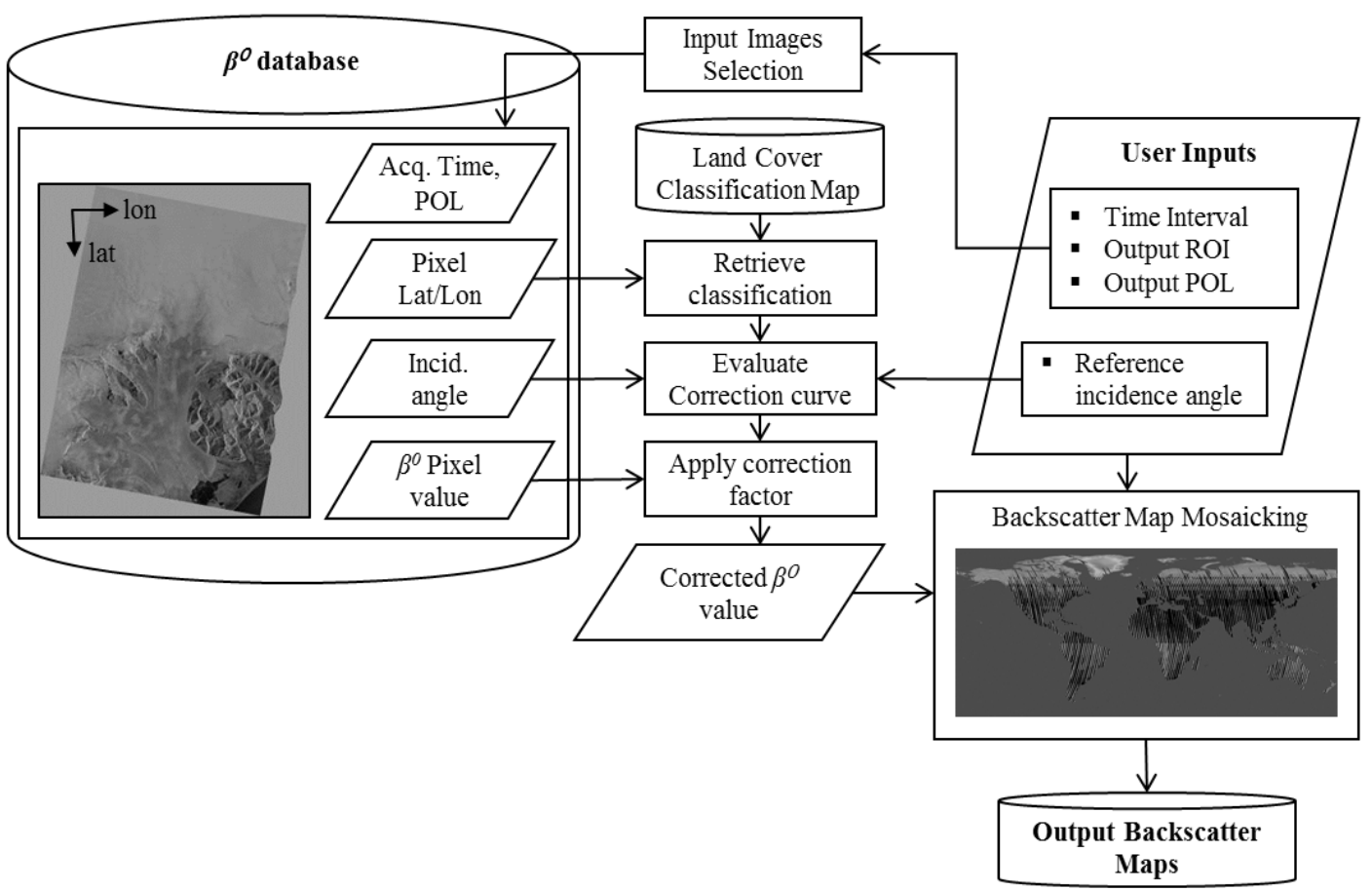

Figure 7.1.: Reference flowchart for the generation of backscatter mosaics, equalized to a defined reference incidence angle $\theta_{\text {ref }}$.

reference. A proper mean $\beta^{0}$ backscatter model is then associated to each GlobCover class.

Data bases of global backscatter models, such as the one derived from TanDEM-X data as in chapter 5 or the one presented F. T. Ulaby and C. Dobson in [38] (from now on simply identified as Ulaby/Dobson data base), can be taken into account for the generation of large-scale mosaics. On the other hand, dedicated models could be applied for the generation of local mosaics.

In this chapter, the Ulaby/Dobson global data base is used as reference since, at the moment, it is the only global one derived for different polarizations, while the TanDEM-X one is provided for HH polarization only. Moreover, the Ulaby/Dobson data base is derived for different bandwidths as well. If necessary, this allows for a closure of remaining gaps within the output map, by using data acquired with different polarizations, as later on explained in section 7.3.

Another aspect that needs to be taken into account when combining the use of the Ulaby/Dobson data base and of the GlobCover map is the availability of a different number of land cover types. In order to solve this problem, several GlobCover classes, which are characterized by similar statistics, are mapped to the same class 
Table 7.1.: GlobCover Vegetation Classes [97] (left) and corresponding Ulaby/Dobson Model [38] (right).

\begin{tabular}{|c|c|}
\hline GLOBCOVER Vegetation Class & Ulaby/Dobson Model \\
\hline Closed to open $(>15 \%)$ broadleaved evergreen or semi-deciduous & \multirow{8}{*}{ Trees } \\
\hline Closed $(>40 \%)$ broadleaved deciduous forest $(>5 \mathrm{~m})$ & \\
\hline Open $(15-40 \%)$ broadleaved deciduous forest/woodland $(>5 \mathrm{~m})$ & \\
\hline Closed $(>40 \%)$ needleleaved evergreen forest $(>5 \mathrm{~m})$ & \\
\hline Open $(15-40 \%)$ needleleaved deciduous or evergreen forest $(>5 \mathrm{~m})$ & \\
\hline Closed to open $(>15 \%)$ mixed broadleaved and needleleaved forest $(>5 \mathrm{~m})$ & \\
\hline Sparse $(<15 \%)$ vegetation & \\
\hline Closed to open (>15\%) broadleaved forest regularly flooded & \\
\hline Post-flooding or irrigated croplands (or aquatic) & \multirow{5}{*}{ Grasses } \\
\hline Rainfed croplands & \\
\hline Mosaic grassland $(50-70 \%)$ / forest or shrubland $(20-50 \%)$ & \\
\hline Closed to open ( $>15 \%)$ herbaceous vegetation (grassland, savannas or lichens/mosses) & \\
\hline Closed to open $(>15 \%)$ grassland or woody vegetation on regularly flooded or waterlogged soil & \\
\hline Mosaic forest or shrubland $(50-70 \%)$ / grassland $(20-50 \%)$ & \multirow{3}{*}{ Shrubs } \\
\hline Closed to open ( $>15 \%)$ (broadleaved or needleleaved, evergreen or deciduous) shrubland & \\
\hline Closed ( $>40 \%$ ) broadleaved forest or shrubland permanently flooded - Saline or brackish water & \\
\hline Artificial surfaces and associated areas (Urban areas $>50 \%$ ) & Roads \\
\hline Bare areas & Soil \& Rocks \\
\hline Permanent snow and ice & Dry Snow \\
\hline Mosaic cropland (50-70\%) / vegetation (grassland/shrubland/forest) (20-50\%) & \multirow{2}{*}{ Short Vegetation } \\
\hline Mosaic vegetation (grassland/shrubland/forest) $(50-70 \%)$ / cropland (20-50\%) & \\
\hline Water bodies & None \\
\hline
\end{tabular}

in the Ulaby/Dobson data base, as presented in Tab. 7.1.

2. The acquisition incidence angle $\theta$ of the considered $\beta^{0}$ pixel is compared to the output reference incidence angle $\theta_{\text {ref }}$ and a correction factor is applied to the input $\beta^{0}$ value. For example, the entire set of Ulaby/Dobson X-band backscatter models, retrieved from [38], are used to implement a series of correction curves as in Fig. 7.2: in (a), the mean $\beta^{0}$ versus the incidence angle for $\mathrm{HH}$ polarization and different ground cover classes is shown; while in (b), for each considered ground cover class, a correction curve $I$ has been computed by inverting such models and considering a reference incidence angle $\theta_{\text {ref }}=40^{\circ}$.

3. Finally, for an input pixel originally acquired with local incidence angle $\theta$ and characterized by a radar brightness equal to $\beta^{0}$, the equalization is performed in $\mathrm{dB}$ as:

$$
\beta_{\theta_{\text {ref }}}^{0}=\beta^{0}+I(\Delta \theta),
$$



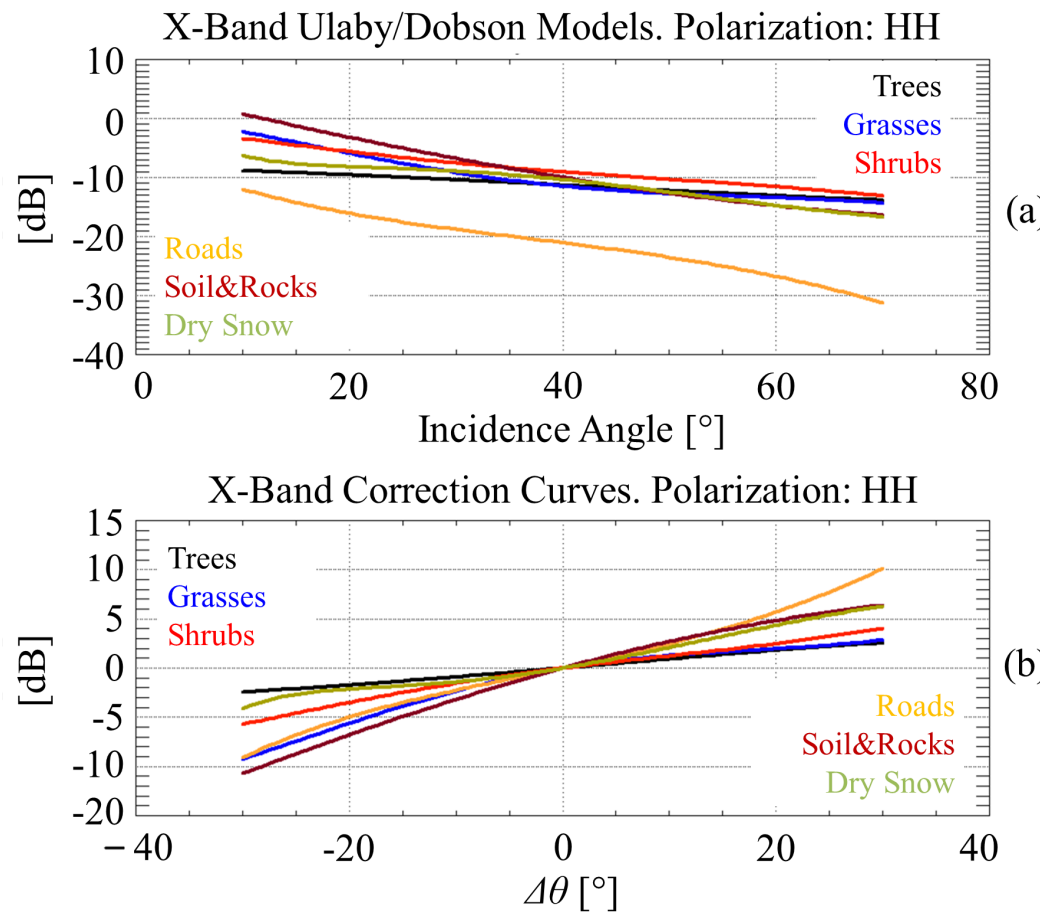

Figure 7.2.: (a) Mean $\beta^{0}$ models for $\mathrm{X}$ band, HH polarization, from the Ulaby/Dobson data base [38]. (b) Correction curves referred to $\theta_{\text {ref }}=40^{\circ}$.

where $\beta_{\theta_{\text {ref }}}^{0}$ represents the pixel radar brightness referred to the output reference incidence angle $\theta_{\text {ref }}, I$ is the incidence angle dependent correction curve associated to the input pixel ground cover class, and $\Delta \theta$ is evaluated as:

$$
\Delta \theta=\theta-\theta_{\text {ref }} .
$$

Depending on its acquisition incidence angle and on the land cover type, it is therefore possible to generate a mosaic which is completely referred to a single incidence angle $\theta_{\text {ref }}$, by applying the proper correction value to each input pixel. In this way, different maps can be generated for several output reference incidence angles, starting from the same input data.

An example is presented in Fig. 7.3, where an equalized mosaic of Iceland, in $\mathrm{HH}$ polarization and referred to an incidence angle $\theta_{\text {ref }}=30^{\circ}$, is generated by composing seven different TerraSAR-X ScanSAR images, acquired from December 2008 to February 2009. 


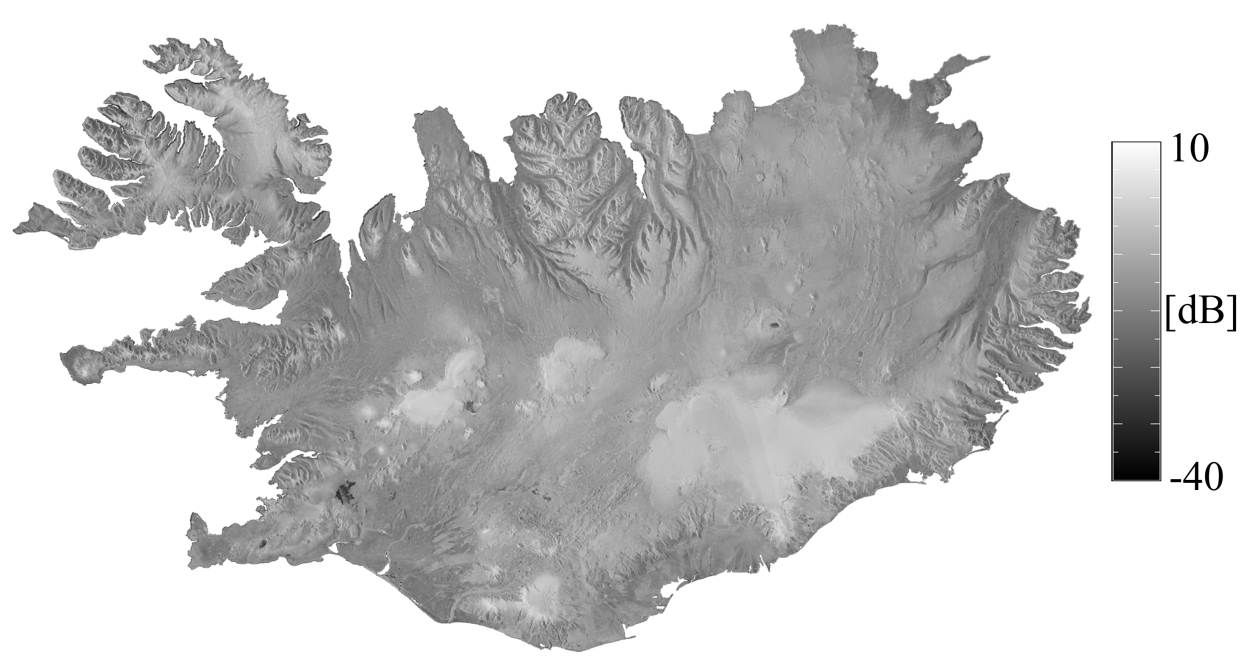

Figure 7.3.: Backscatter Mosaic of Iceland generated using seven TerraSAR-X ScanSAR images, acquired from December 2008 to February 2009. Polarization: HH, reference incidence angle $\theta_{\text {ref }}=30^{\circ}$, resolution: $200 \mathrm{~m} \times 200 \mathrm{~m}$, orbit direction: ASC.

Note that more than one input $\beta^{0}$ pixel value, e.g. from acquisitions at different dates, can contribute to the same output cell within the backscatter map to be generated. For this reason different ways to combine multiple input pixels are considered during the mosaicking process and the following output maps are generated:

- Backscatter mean value map: obtained by averaging all the $n$ available calibrated and interpolated $\beta_{\theta_{\text {ref }}}^{0}(k)$ values inside the single output resolution cell (where $k=$ $[1, \ldots, n])$.

- Backscatter maximum value map: evaluated as the maximum value of all the available $\beta_{\theta_{\text {ref }}}^{0}(k)$ contributing to the same output cell.

- Backscatter standard deviation map: evaluated as the sample standard deviation of all the available $\beta_{\theta_{\text {ref }}}^{0}(k)$ values inside the considered output cell. A default value equal to zero is set if only one input pixel is available inside the output cell.

- Type mask: complementary information about the type of input data used for the generation of the output backscatter map is provided by this map, giving information about the type of input data (e.g. mission, polarization, etc.).

Each map can be useful for different purposes. For example, the Backscatter mean value map can be used for performance estimation (SNR, height error, etc.), the Backscatter maximum value map for saturation level evaluation (in order to avoid clipping), the 


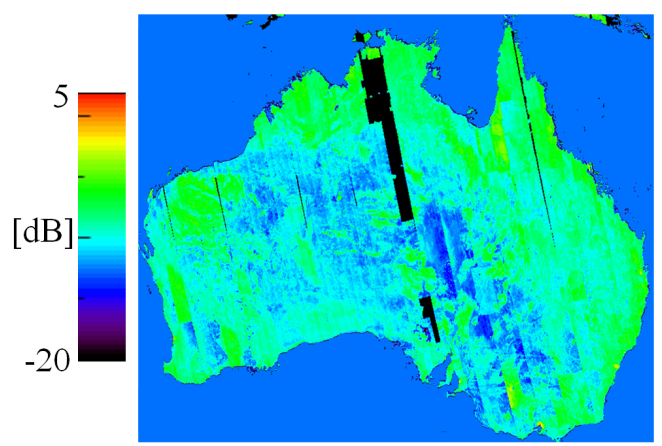

(a)

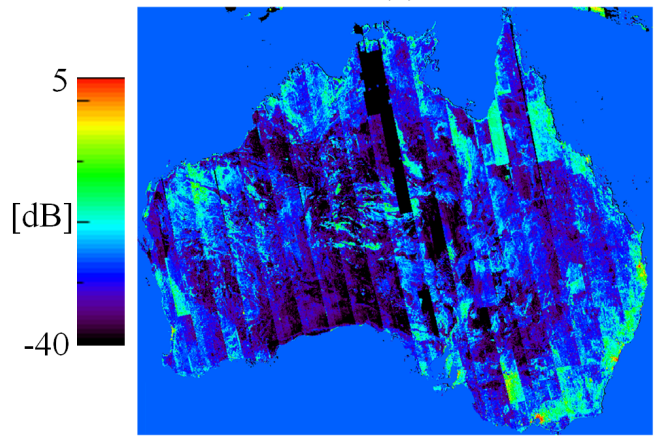

(c)

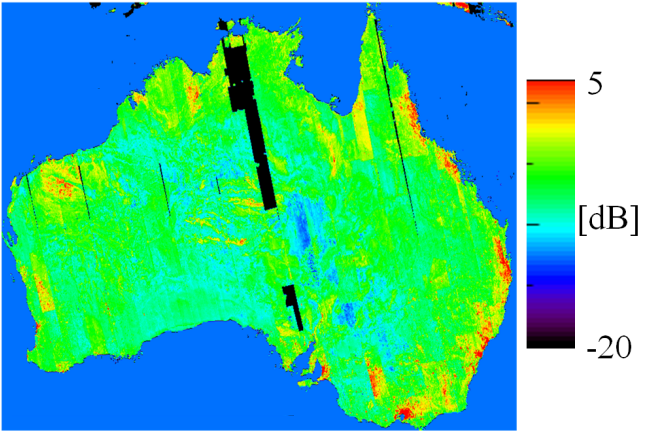

(b)
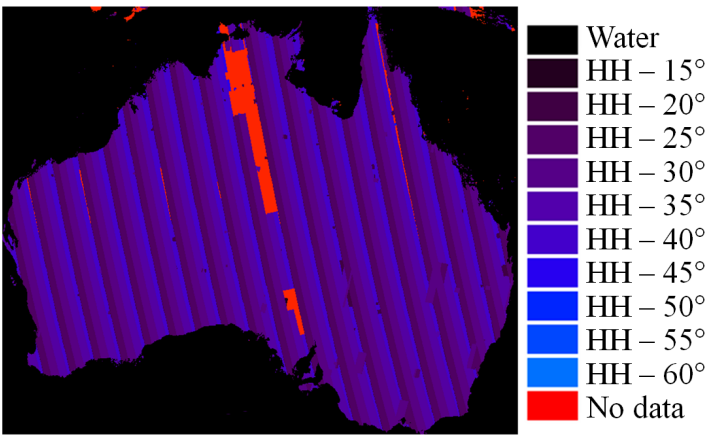

(d)

Figure 7.4.: TerraSAR-X $\beta^{0}$ backscatter map example over Australia, HH pol, $\theta_{\text {ref }}=$ $30^{\circ}$. (a) Backscatter mean value map. (b) Backscatter maximum value map. (c) Backscatter standard deviation map. (d) Type mask.

Backscatter standard deviation map for analyzing the image dynamic range and its impacts on data compression rate [24], and the Type mask for a statistical analysis of the mosaicked input SAR data.

Fig. 7.4 shows an example of a backscatter map over Australia generated using SAR data from the TerraSAR-X mission, acquired from 2009 to 2010. It is evaluated for $\mathrm{HH}$ polarization, a reference incidence angle $\theta_{\text {ref }}=30^{\circ}$, and angular sampling of $0.05^{\circ}$ in latitude/longitude coordinates, which corresponds to a ground resolution of about $5 \mathrm{~km}$ $\times 5 \mathrm{~km}$ at the Equator. Missing values over land areas in Fig. 7.4 (a), (b), and (c) are associated to the default value of $-20 \mathrm{~dB}$, which corresponds to the typical TerraSAR$\mathrm{X}$ noise floor. In Fig. 7.4 (d), the corresponding Type mask is presented. Each color classifies the input pixels used for generating the output mosaics, and in particular:

- Water bodies are masked off during the generation process, according to the information provided by the GlobCover, and associated to a default value (colorbar class name: Water). 
- Input SAR data are classified depending on the acquired polarization and incidence angle. Different colors correspond to a series of 10 reference incidence angle intervals. Each interval is identified by its mean value, starting from $15^{\circ}$ until $60^{\circ}$, and is characterized by a spread of $\pm 2.5^{\circ}$.

- If real SAR data are missing over land areas, output cells values are identified as No data.

Note that in Fig. 7.4 (a), the averaging process of the input pixels results in a significant reduction of the backscatter dependence on the acquisition polarization and incidence angle. However, few discontinuities are still present due to the limited number of available input data (in most of the cases only one single coverage was provided for averaging). With more overlapping coverages this effect can be further reduced. In Fig. 7.4 (b) and (c), these phenomena are more visible, since no averaging process is performed.

The quality of the final backscatter map depends on several factors, such as:

- the radiometric calibration accuracy of input SAR products.

- the geocoding accuracy.

- the accuracy and representativeness of the used land cover classification map (in this case, the GlobCover).

- the accuracy of the applied correction curves.

- the availability of input SAR data.

If TerraSAR-X and TanDEM-X products are considered as input data, the first two factors are not expected to significantly influence the final global maps quality, given the high accuracy in both absolute calibration and geocoding [60], [79], [80]. Due to temporal surface changes, an incorrect ground cover classification can be associated to the considered area, leading to the application of an incorrect correction curve. Moreover, the correction curves are evaluated taking into account mean $\beta^{0}$ models only, without considering their dispersion from the expected value. However, in the presented examples, since the standard deviation of the Ulaby/Dobson models does not significantly change with respect to the incidence angle [38], no significant error is expected to be introduced, even when the correction curves are applied to backscatter values that substantially differ from the correspondent Ulaby/Dobson model mean value. Finally, more real input SAR data leads to a better averaging of backscatter pixels inside the same output cell. 


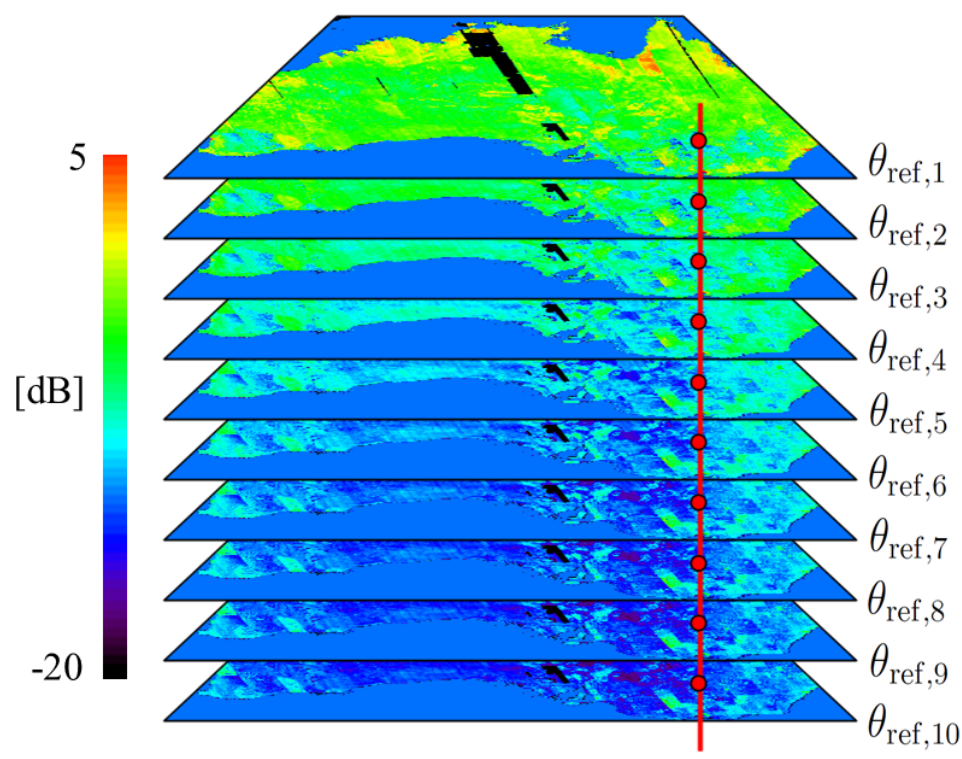

Figure 7.5.: Example of output backscatter map product structure, consisting of a stack of ten $\beta^{0}$ backscatter maps, referred to ten different output reference incidence angles $\theta_{\text {ref }, n}[n=1, \ldots, 10]$, for a single polarization channel $(\mathrm{HH})$. The red vertical line indicates the direction of a linear interpolation, which can be performed to retrieve a backscatter map for any desired reference incidence angle $\theta_{\text {ref }}$ between $\theta_{\text {ref }, 1}$ and $\theta_{\text {ref }, 10}$.

\subsection{Global Backscatter Map Final Structure}

By applying the proper correction curves, a stack of 10 different output backscatter maps for each polarization $(\mathrm{HH}, \mathrm{VV}$, and $\mathrm{HV} / \mathrm{VH})$ is generated, corresponding to ten different output reference incidence angles $\theta_{\text {ref, } n}$ (with $n=1, \ldots, 10$ ), as shown in Fig. 7.5. An overall incidence angle span from $15^{\circ}$ to $60^{\circ}$ is considered, and backscatter maps at $5^{\circ}$ step are generated. In this example, the input pixels, contributing to each output map, were acquired with the same polarization as the output backscatter map and with an incidence angle within a spread of $\pm 2.5^{\circ}$ from the output backscatter map reference incidence angle $\theta_{\text {ref }}$.

The final global backscatter map has to allow for the retrieval of a $\beta^{0}$ value for any required polarization and incidence angle within the defined overall incidence angle interval. For the required polarization, the user can finally perform a linear interpolation, in order to retrieve a backscatter value referred to a specific reference incidence angle, which differs from any of the already available $\theta_{\text {ref }, n}$ within the stack. 


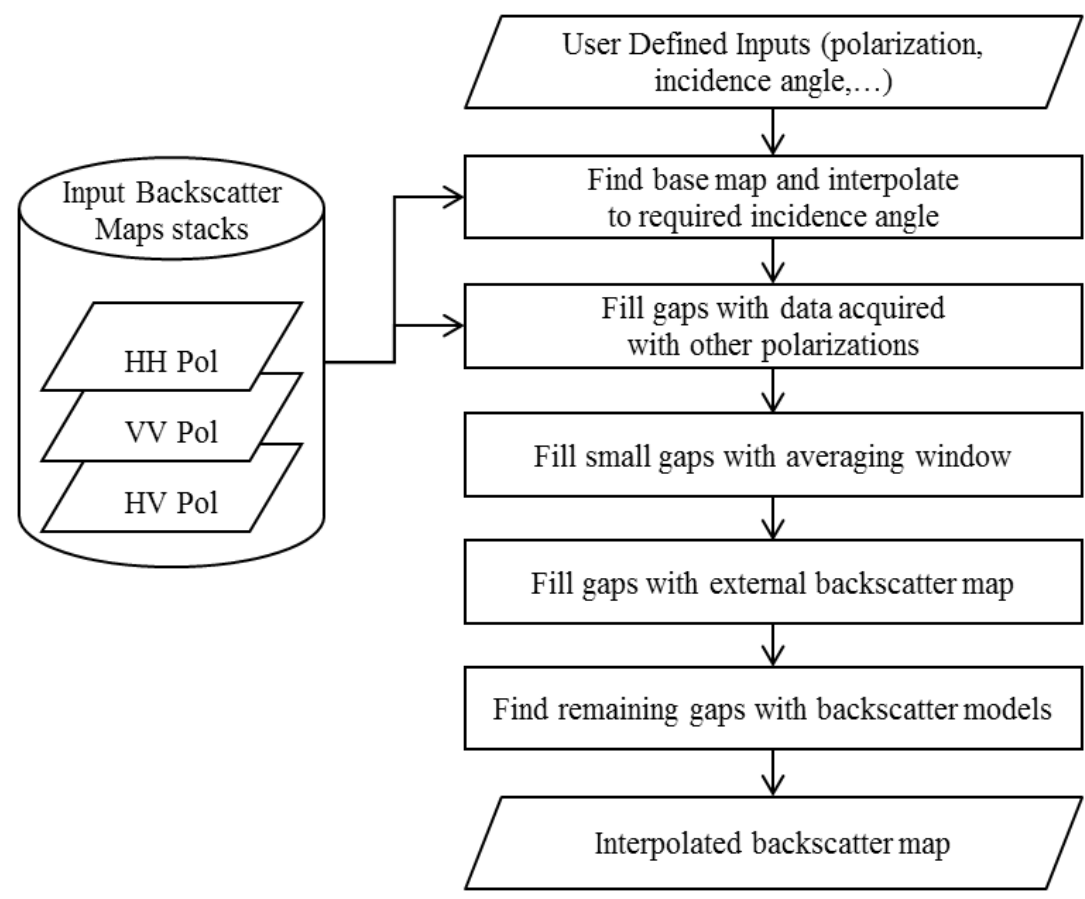

Figure 7.6.: Filling of missing values. Algorithm flowchart.

\subsection{Filling of Missing Values}

If a global coverage of input SAR data is not available, the generated backscatter map will be affected by the presence of gaps. For many applications requiring the use of backscatter maps, such as commanding or SAR system design, such gaps need to be closed.

In this section, a dedicated algorithm for filling the empty output cells is presented. The reference flowchart is depicted in Fig. 7.6. Several steps are subsequently performed until the complete coverage is achieved:

1. The first step consists in the selection of the so-called base map: once the desired output polarization and reference incidence angle $\theta_{\text {ref }}$ have been chosen, the corresponding backscatter map is selected from the stack of backscatter maps in Fig. 7.5. If the reference incidence angle of this base map differs from the desired output one, all pixels are interpolated to the required output incidence angle as explained in section 7.2.

2. The second step consists in using available input SAR data, acquired with different polarizations, and interpolating them to the desired polarization and reference incidence angle using a set of correction curves, as presented in section 7.1. 
3. In the third step, small gaps are filled by averaging the nearest available samples inside a defined two-dimensional window, assuming that backscatter characteristics are similar among nearby pixels.

4. The fourth step consists in filling larger gaps by using data retrieved from external available backscatter maps. Also in this case, such data has to be referred to the proper polarization and incidence angle, by applying the considered set of correction curves.

5. Finally, the fifth steps allows for the closure of remaining gaps over regions where no external backscatter map is available. For each considered pixel, its land cover type is retrieved and the corresponding the backscatter model, evaluated for the output reference incidence angle, is directly used.

The standard deviation map (described in section 7.1) is set to a default value equal to 0 if no real SAR data are used for the considered output cell.

An example of the proposed algorithm is presented in Fig. 7.7. Fig. 7.7 (a) shows the backscatter mean value map, generated by using TerraSAR-X data only, acquired in $\mathrm{HH}$ polarization from October 2008 to April 2010. It has a pixel spacing of $0.05^{\circ}$ in latitude/longitude coordinates. As can be seen, many regions, particularly over North America, Asia, and Antarctica, present data gaps (black areas).

The resulting backscatter mean value map after the application of the algorithm for filling missing values is shown in Fig. 7.7 (b). In this case, a C-band backscatter map provided by the ESA [149], generated for $\mathrm{HH}$ polarization and referred to an incidence angle of $30^{\circ}$, was used to implement the algorithm's step 4. An equalization process, similar to the one described in section 7.1, has been applied to the C-band mosaic, in order to convert it from $\mathrm{C}$ band to $\mathrm{X}$ band first, and then to the required output reference incidence angle. In this case, a multi-frequency set of backscatter models is required and, again, the Ulaby/Dobson backscatter data base was used to implement the correction curves.

The considered C-band mosaic does not provide a global coverage of the Earth: it is defined for latitudes between $-60^{\circ}$ and $60^{\circ}$ and not all land masses are mapped (e.g. Madagascar and Indonesia are missing). Therefore, the Ulaby/Dobson mean backscatter models and the GlobCover classification map have been finally applied to implement the algorithm's step 5, in order to close all remaining gaps.

Finally, the Type Mask associated to the mosaic in Fig. 7.7 (b), is depicted in Fig. 7.7 (c). Each color corresponds to a different kind of data used for the generation of the final mosaic, as explained in section 7.1. For the sake of clarity TerraSAR-X data values are grouped together depending on the polarization only (TSX HH-Pol, TSX VV-Pol, and TSX HV-Pol classes) and no further details on the specific incidence angle interval are presented. The values Window, Cband, and Ulaby in Fig. 7.7 (c) identify the 3 different 
(a)

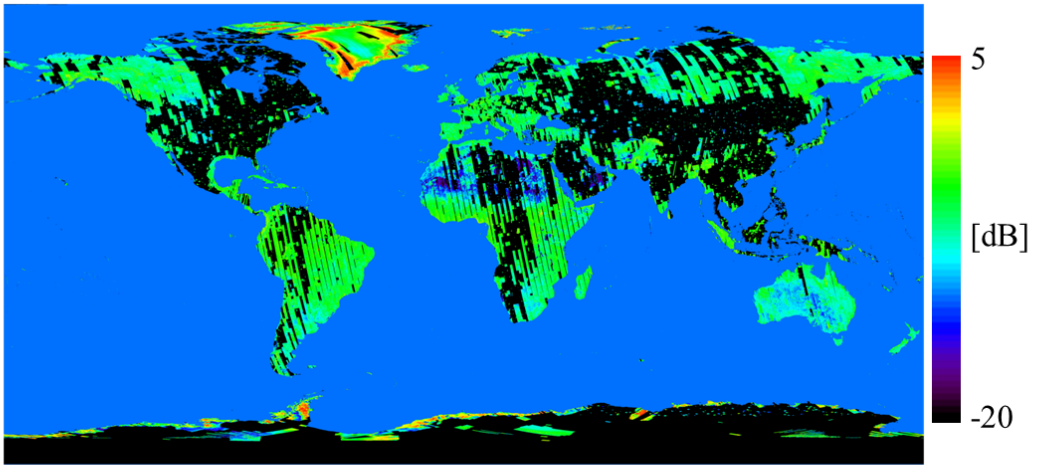

(b)

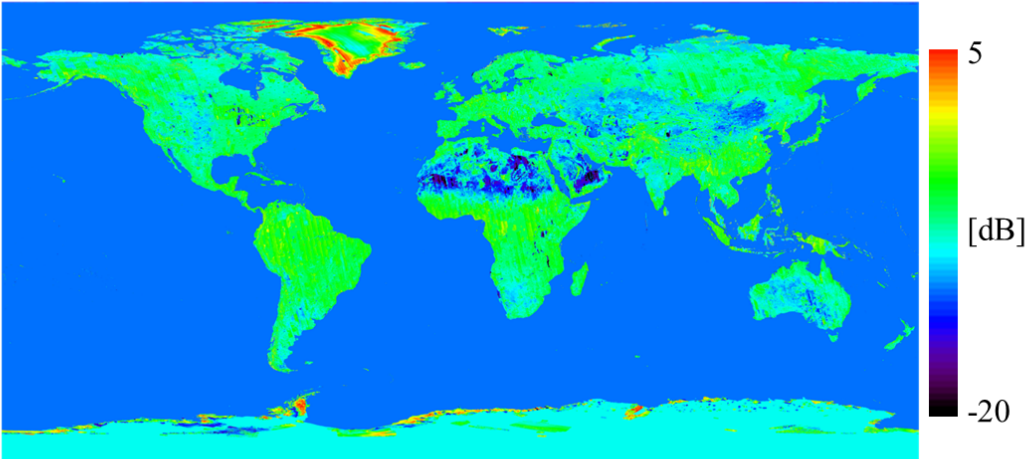

(c)

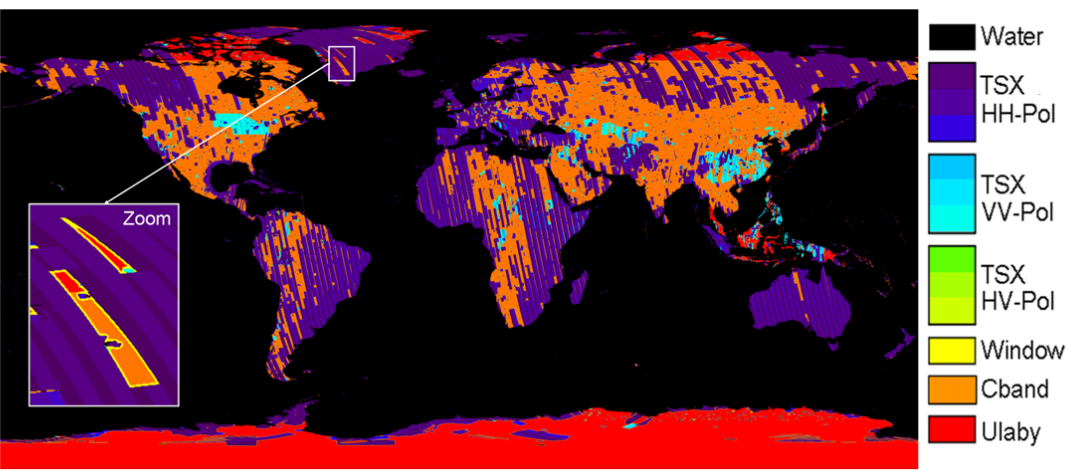

Figure 7.7.: (a) $\beta^{0}$ mean value for $\mathrm{HH}$ polarization, referred to $30^{\circ}$ incidence angle, generated using TerraSAR-X data in HH polarization only and different incidence angles. (b) $\beta^{0}$ mosaic after filling data gaps using the interpolation algorithm presented in section 7.3. (c) Type Mask associated to the backscatter map in (b).

approaches for filling missing values presented at step 3, 4, and 5, respectively. 


\section{Conclusions and Future Work}

\section{Summary and Conclusions}

In this work, a new method for the characterization of the radar backscatter, using a global statistical approach, has been presented. Given the global database of X-band SAR images, acquired within the TanDEM-X mission, and the introduction of the unique topographic information associated to them, it is possible to derive an up-to-date set of statistical X-band backscatter models for the 21 valid classes of the GlobCover classification map (excluded invalid and permanent snow and ice, which has been separately addressed in chapter 6), depending on the acquisition parameters and, particularly, on the incidence angle.

Two different models for the radar brightness have been derived, using the same estimation approach once not including (unweighted modeling) and later including (weighted modeling) the reliability of the input measurements, respectively. The associated quality weights depend on the number of available input observations and on the similarity of the estimated radar brightness histogram with respect to a theoretical Normal distribution. As needed for a varying number of scientific applications, $\sigma^{0}$ and $\gamma^{0}$ models can be directly derived from the $\beta^{0}$ models.

About 240,000 acquired scenes have been considered, allowing for the generation of individual models for each GlobCover map class. For a certain class, TanDEM-X images acquired all over the world and characterized by the presence of at least one sample classified according to the considered class are taken into account, allowing for a global modeling approach. The large number of classes provided by the GlobCover increases the level of precision in terms of classification, compared to global backscatter models data bases provided so far. As an example, boreal forests and rainforests can be clearly discriminated and modeled using TanDEM-X data acquired over the correct ground areas, while vegetated areas in the widely used Ulaby/Dobson backscatter models data base [38] are simply characterized as Trees, and modeled using ground measurements on boreal forests only.

The developed verification approach for the derived models has been presented as well, based on comparing overlapping TanDEM-X acquisitions over dedicated test sites. In particular, the verification of the closed to open $(>15 \%)$ broadleaved evergreen or semideciduous forest ( $>5 \mathrm{~m}$ ) GlobCover class has been detailed: 10 test sites have been used to verify both backscatter models, which have been derived in section 5.3.2 using the global data set from the TanDEM-X mission data. The obtained results show that the use of quality weights to be associated to the input measurements allows for the derivation 
of more reliable backscatter models, with both curvature and slope accuracy better than $1 \mathrm{~dB}$.

The large majority of the available TanDEM-X data are currently being acquired using $\mathrm{HH}$ polarization, which is the one used for the generation of the global DEM. The extension of the derived backscatter models to other polarizations, by using dedicated TanDEM-X or other sensors acquisitions, is a further objective of the current research and will be analyzed in the future.

A refinement in the technique can also be done by discriminating areas affected by geometric distortion, due to shadow and layover, in a more accurate way, e.g. by exploiting the topographic information from the global TanDEM-X DEM.

$\mathrm{X}$-band radar backscatter behavior over rainforests from TanDEM-X data has been specifically analyzed by using $\gamma^{0}$ mosaics, generated by properly composing TanDEM-X quicklook data. Rainforests appear to be quite stable and near-isotropic targets, with backscatter stabilities in the order of $0.3 \mathrm{~dB}$ standard deviation. A slight decrease of $\gamma^{0}$ levels, with respect to the incidence angle of illumination, was detected from $\gamma^{0}$ profiles over the Amazon rainforest in descending acquisition geometry. Moreover, rainforest backscatter in $\mathrm{X}$ band is mostly influenced by the day time of acquisition. This fact can be explained by the dependency of X-band radar backscatter on the presence of water on the tree leaves (or, as a secondary effect, on the level of humidity of the air), which can be a function of day time.

The stability of the $\gamma^{0}$ profiles represents a necessary condition for performing a reliable monitoring of the SAR elevation antenna pattern, by exploiting the knowledge of rain forests radar cross section. The analyzed aspects have therefore to be taken into account when choosing the appropriate acquisitions for SAR calibration purposes.

A data base of backscatter models is provided in appendix A. For each GlobCover class, a set of statistical parameters is provided, derived using data acquired over one single continent only. The continent's choice was based on both the number of available observations and on how representative such a continent is for the considered ground cover class. Moreover, backscatter statistics and corresponding fitting coefficients are always presented for two different seasons: winter and summer.

This data set will provide a valuable basis for investigations on X-Band backscatter behavior from different SAR sensors, for the optimization of future SAR missions, and for many other scientific applications. Moreover, the presented modeling technique is also applicable to other current and future SAR mapping missions, even at different frequency bands, such as Tandem-L [77], Sentinel-1 [19], and ALOS PALSAR [14].

In chapter 6, an approach for locating different snow facies of the Greenland Ice Sheet by exploiting X-band TanDEM-X interferometric SAR acquisitions has been presented. An unsupervised classification method based on the $c$-means fuzzy clustering algorithm has been applied, which uses features inherent in the data without subjective interfer- 
ence. This is an appropriate method for exploring the information content of the 2D feature space, given by the combination of radar backscatter $\gamma^{0}$ and volume correlation factor $\gamma_{\mathrm{Vol}}$, with respect to glacier facies, which is a main objective of the work. The algorithm has been applied to TanDEM-X data acquired during winter 2010/2011, by analyzing three different partitions, obtained by selecting a different number of clusters $(c=3,4,5)$, in order to assess the feasibility for discriminating facies types. The partition composed of 4 clusters is a good compromise in terms of classification reliability and high level of detail and has therefore been chosen as reference for the current work. A statistical analysis of both $\gamma^{0}$ and $\gamma_{\text {Vol }}$ over the Ice Sheet for each different facies has been provided, together with an analysis on the dependency of $\gamma_{\mathrm{Vol}}$ on the acquisition geometry and, in particular, on the height of ambiguity ranging from $40 \mathrm{~m}$ to $53 \mathrm{~m}$. The use of a correction factor for $\gamma_{\mathrm{Vol}}$ depending on the height of ambiguity might represent a starting point for a future refinement of the classification algorithm. The derived snow facies have been interpreted by means of reference melt data and in situ measurements along the EGIG line. Facies 1 is dominated by the presence of dry snow. Further refined clustering reveals two sub-facies (a southern and a northern one) which can be related to different snow accumulation rates. Facies 2 to 4 belong to a transition zone where melt phenomena increase toward the outer regions of the Ice Sheet. Facies 2 and 3 approximately correspond to the percolation zone, and facies 4 to the wet snow zone, reported by Benson in [103]. This is confirmed by structural properties of the snow volume as observed by Morris and Wingham in [141]. The subdivision into different facies results from differences in $\gamma^{0}$ and $\gamma_{\mathrm{Vol}}$ due to spatial changes in microstructure of firn related to melt intensity and accumulation rates, which vary with elevation, snowfall pattern, and wind drift. The subdivision is therefore a pointer to such differences.

The availability of a refined classification map allowed then to apply a modified version of the algorithm presented in chapter 5, resulting in a different incidence-dependent backscatter model for each snow facies.

Finally, given the high similarity in terms of backscattering properties and volume decorrelation among pixels belonging to the same cluster, one can then apply the mean value of snow density to the entire considered snow facies. This allowed to estimate the penetration depth by inverting the interferometric model proposed by Weber Hoen and Zebker in [119] and assuming the dielectric constant for an icy medium to be real and to remain constant for a given facies type. The obtained results show a mean two-way penetration depth of $4.18 \mathrm{~m}$ for facies $1,3.58 \mathrm{~m}$ for facies $2,3.07 \mathrm{~m}$ for facies 3, and $2.34 \mathrm{~m}$ for facies 4 . These values have been compared to the elevation difference between the global TanDEM-X DEM and ICESat measurements, proving that, theoretically, no considerable bias between the two measurement approaches is to be expected. A residual negative offset has nevertheless been detected, which varies from about $-0.8 \mathrm{~m}$ to -1.40 $\mathrm{m}$ for the different snow facies, which will be objective of further investigations. A possible explanation might be the fact that the Weber Hoen and Zebker's model relies on simplifying assumptions, such as no depth dependency of the radar cross-section, a con- 
stant density, and uncorrelated scatterers. Other sources of uncertainty may be related to the TanDEM-X DEM calibration or to the occurrence of height changes during the time span which separates ICESat measurements from TanDEM-X acquisitions.

Finally, in chapter 7, an algorithm for the derivation of backscatter maps has been presented. It requires the use of a data set of backscatter models in order to equalize the output mosaic to a certain reference incidence angle and polarization. If a complete coverage of the desired output region of interest with real SAR data are not available, missing values need to be filled. An iterative algorithm has been proposed, in order to fill gaps with corrected data acquired with other incidence angles and polarizations, values coming from neighbor samples, or even data acquired with a different sensors.

The generation of backscatter maps has been discussed using spaceborne SAR data from the TerraSAR-X and TanDEM-X missions.

\section{Future Work and Outlook}

The developed algorithms and models described in this thesis represent a powerful starting point for the development of new scientific products and techniques.

First of all, in order to fully exploit the capabilities of the TanDEM-X data set, a future activity will be the generation of a global X-band backscatter map using TanDEM-X data only, equalized using the data base of mean backscatter models presented in this work.

The developed algorithm for modeling backscatter allows for the generation of output models, which can serve different purposes, from SAR system design to performance monitoring. Moreover, the flexible choice of input data allows for the generation of backscatter mosaics which can be used for several scientific investigations. For example, mosaics generated using input data acquired during pre-defined seasons are an effective tool for monitoring backscatter evolution in time or its dependency on soil conditions. In this sense, this work opens the door for the generation of adaptive backscatter maps, which can dynamically take into account weather and seasonal conditions. This goes in the direction of cognitive radars, which will probably be the next generation of adaptive radars, characterized by an unprecedented capability to intelligently adapt the operation and performance to the surrounding environment [150]. For example, geographic- and seasonal-dependent backscatter maps could serve as prior knowledge of the environment for the development of novel data compression techniques, oriented toward the exploitation of variable data rates and optimized depending on both the desired scientific application and the acquisition conditions [151].

The developed research work for identifying different snow facies of the Greenland Ice Sheet represents a starting point for further analyzing the evolution of ice sheets, by monitoring the changes in the location of the different snow facies, as an indicator of climate changes. Moreover, even though featuring a limited penetration into the snow pack, 
TanDEM-X interferometric data demonstrates itself to be highly sensitive to changes in the snow properties and represents a highly valuable data set for investigating Greenland Ice Sheet characteristics and its evolution. The continuous monitoring of the cryosphere in an era of climate changes represents one of the most challenging tasks for the remote sensing community. Additionally, the developed approach can also be applied to more recent TanDEM-X time-tagged acquisitions over Greenland and Antarctica Ice Sheets, in order to provide an up-to-date monitoring of their properties. Most importantly, the technique could be exploited within future interferometric SAR missions as well. For example, the Tandem-L mission is being currently designed for acting as single-pass interferometer at L band [77], with the main objective of observing the dynamic processes in the Earth's environmental system.

It is finally worth noting that the knowledge and experience gained on machine learning classification methods, established in the framework of the Greenland Ice Sheet activities, set the basis for the generation of the global TanDEM-X Forest/Non-Forest Map [32]. The latter is the first global forest mapping product derived from bistatic interferometric spaceborne SAR data. It is based on the exploitation of the volume correlation factor, derived from the interferometric coherence, which is a very sensitive and reliable indicator of the presence of vegetation on ground.

The global product and the developed algorithms are now freely available for the scientific community, to be used for a large variety of applications, such as forest mapping and deforestation monitoring [152], [33].

The intent is now to first enhance the global TanDEM-X Forest/Non-Forest Map into a more generalized high-resolution land cover classification map, by adding additional layers, such as water bodies, urban areas, deserts, or snow- and ice-covered regions. Second, going towards the era of global satellite missions and big data, these activities aim at investigating the potentials of machine learning and deep learning applied to remote sensing data for the extraction of geophysical parameters. 


\section{Bibliography}

[1] F. T. Ulaby and D. G. Long, Microwave Radar and Radiometric Remote Sensing. The University of Michigan Press, 2014.

[2] R. Bamler and P. Hartl, Synthetic Aperture Radar Interferometry. Inverse Problems 14. 1998.

[3] A. Ferretti, A. Monti Guarnieri, C. Prati, and F. Rocca, "InSAR Principles: Guidelines for SAR Interferometry Processing and Interpretation,” Technical Manual TM-19, European Space Agency (ESA), 2007.

[4] A. Reigber and A. Moreira, "First Demostration of Airborne SAR Tomography Using Multibaseline L-Band Data," IEEE Transactions on Geoscience and Remote Sensing, vol. 38, pp. 2142-2152, Sep. 2000.

[5] J. Lee and E. Pottier, Polarimetric Radar Imaging. Boca Raton, USA: CRC Press, Taylor \& Francis Group, 2009.

[6] W. M. Boerner, L. A. Cram, W. A. Holm, D. E. Stein, W. Wiesbeck, W. Keydel, D. Giuli, D. T. Gjessing, F. A. Molinet, and H. Brand, Direct and Inverse Methods in Radar Polarimetry. New York, USA: Springer Science \& Business Media, 1992.

[7] H. R. Hertz, "Über Sehr Schnelle Electrische Schwingungen," Annalen der Physik, vol. 267, no. 7, pp. 421-448, 1887.

[8] C. Hülsmeyer, “The Telemobiloscope,” Electrical Magazine, vol. 2, p. 388, 1904.

[9] A. W. Love, "In Memory of Carl A. Wiley," IEEE Antennas and Propagation Society Newsletter, vol. 27, pp. 17-18, Jun. 1985.

[10] F. Lee-Lueng and B. Holt, "Seasat Views Oceans and Sea Ice With Synthetic Aperture Radar," Publication 81-120, NASA, JPL, Feb. 1982.

[11] J. Cimino and C. Elachi, "Shuttle Imaging Radar-A (SIR-A) Experiment," Publication 8277, NASA, JPL, 1982.

[12] C. Elachi, "Shuttle Imaging Radar Experiment," Science, vol. 218, no. 4576, pp. 996-1003, 1982. 
[13] J. Cimino, C. Elachi, and M. Settle, "SIR-B - The Second Shuttle Imaging Radar Experiment," IEEE Transactions on Geoscience and Remote Sensing, vol. GE-24, pp. 445-452, Jul. 1986.

[14] A. Rosenqvist, M. Shimada, and M. Watanabe, "ALOS PALSAR: A Pathfinder Mission for Global-Scale Monitoring of the Environment," IEEE Transactions on Geoscience and Remote Sensing, vol. 45, pp. 3307-3316, Nov. 2007.

[15] Y. Okada, S. Nakamura, K. Iribe, Y. Yokota, M. Tsuji, K. Hariu, Y. Kankaku, S. Suzuki, Y. Osawa, and M. Shimada, "System Characteristics for Wide Swath L-Band SAR onboard ALOS-2/PALSAR-2," in Proceedings of the Asia-Pacific Conference on Synthetic Aperture Radar (APSAR), (Tsukuba, JP), pp. 141-143, Sep. 2013.

[16] R. Francis and al., "The ERS-1 Spacecraft and its Payload," ESA Bulletin, pp. 2748, Feb. 1991.

[17] R. Francis and al., "The ERS-2 Spacecraft and its Payload," ESA Bulletin, pp. 1331, Aug. 1995.

[18] J. Louet, “The Envisat Mission and System,” ESA Bulletin, pp. 10-25, Jun. 2001.

[19] P. Potin, B. Rosich, J. Roeder, and P. Bargellini, "Sentinel-1 Mission Operations Concept," in Proceedings of the IEEE Geoscience and Remote Sensing Symposium (IGARSS), (Quebec City, CA), pp. 1465-1468, Jul. 2014.

[20] A. Mamhood, "RADARSAT-1 Background Mission Monitoring of the Arctic," in Proceedings of the IEEE Geoscience and Remote Sensing Symposium (IGARSS), vol. 1, (Seoul, KOR), pp. 5-8, Jul. 2005.

[21] A. Hillman, P. Rolland, M. Chabot, R. Periard, P. Ledantec, and N. Martens, "RADARSAT-2 Mission Operations Status," in Proceedings of the IEEE Geoscience and Remote Sensing Symposium (IGARSS), (Vancouver, CA), pp. 34803484, Jul. 2011.

[22] "Shuttle Imaging Radar Mission Web Page," Apr. 2018. Available at http: //science.nasa.gov/missions/sir/.

[23] M. Werner, "Shuttle Radar Topography Mission (SRTM): Mission Overview," J. Telecommun. (Frequenz), vol. 55, no. 3/4, pp. 75-79, 2001.

[24] J. Mittermayer, M. Younis, R. Metzig, S. Wollstadt, J. Márquez, and A. Meta, "TerraSAR-X System Performance Characterization and Verification," IEEE Transactions on Geoscience and Remote Sensing, vol. 48, pp. 660-676, Feb. 2010. 
[25] G. Krieger, A. Moreira, H. Fiedler, I. Hajnsek, M. Werner, M. Younis, and M. Zink, "TanDEM-X: A Satellite Formation for High-Resolution SAR Interferometry," IEEE Transactions on Geoscience and Remote Sensing, vol. 45, pp. 3317-3341, Nov. 2007.

[26] F. Caltagirone, G. De Luca, F. Covello, G. Marano, G. Angino, and M. Piemontese, "Status, Results, Potentiality and Evolution of COSMO-SkyMed, the Italian Earth Observation Constellation for Risk Management and Security," in Proceedings of the Geoscience and Remote Sensing Symposium (IGARSS), (Honolulu, USA), pp. 4393-4396, Jul. 2010.

[27] S.-R. Lee, "Overview of KOMPSAT-5 Program, Mission, and System," in Proceedings of the Geoscience and Remote Sensing Symposium (IGARSS), (Honolulu, USA), pp. 797-800, Jul. 2010.

[28] G. Krieger, I. Hajnsek, K. Papathanassiou, M. Eineder, M. Younis, F. De Zan, P. Prats, S. Huber, M. Werner, H. Fiedler, A. Freeman, P. Rosen, S. Hensley, W. Johnson, L. Veilleux, B. Grafmueller, R. Werninghaus, R. Bamler, and A. Moreira, "The Tandem-L Mission Proposal: Monitoring Earth's Dynamics with High Resolution SAR Interferometry," in Proceedings of the Radar Conference, IEEE, (Pasadena, USA), pp. 1-6, May. 2009.

[29] M. Younis, C. Fischer, and W. Wiesbeck, "Digital Beamforming in SAR Systems," IEEE Transactions on Geoscience and Remote Sensing, vol. 41, pp. 1735-1739, Jul. 2003.

[30] M.-L. Truong-Lot, P. Dubois-Fernandez, and E. Pottier, "Compact Polarimetry Potentials," in Proceedings of the IEEE Geoscience and Remote Sensing Symposium (IGARSS), (Vancouver, CA), pp. 3823-3826, Jul. 2011.

[31] H. Rott, T. Nagler, K. Voglmeier, M. Kern, G. Macelloni, M. Gai, U. Cortesi, R. Scheiber, I. Hajnsek, J. Pulliainen, and D. Flach, "Algorithm for Retrieval of Snow Mass from Ku- and X-Band Radar Backscatter Measurements," in Proceedings of the IEEE Geoscience and Remote Sensing Symposium (IGARSS), (Munich, GER), pp. 135-138, Jul. 2012.

[32] M. Martone, P. Rizzoli, C. Wecklich, C. Gonzalez, J.-L. Bueso-Bello, P. Valdo, D. Schulze, M. Zink, G. Krieger, and A. Moreira, "The Global Forest/Non-Forest Map from TanDEM-X Interferometric SAR Data," Remote Sensing of Environment, vol. 205, pp. 352-373, Feb. 2018.

[33] P. Rizzoli, J. Bueso-Bello, A. Pulella, F. Sica, and M. Zink, "A Novel Approach to Monitor Deforestation in the Amazon Rainforest by Means of Sentinel-1 and 
TanDEM-X Data," in Proceedings of the Geoscience and Remote Sensing Symposium (IGARSS), 2018, Accepted Paper.

[34] I. S. Grant and W. R. Phillips, Electromagnetism. John Wiley \& Sons, 1990.

[35] M. Skolnik, Radar Handbook. New York, USA: McGraw-Hill, third ed., 2008.

[36] "Image of public domain retrieved from wikipedia," Apr. 2018. Available at https://id.wikipedia.org/wiki/Berkas:Circular. Polarization.Circularly.Polarized.Light_Circular. Polarizer_Creating.Left.Handed.Helix.View.svg.

[37] L. Tsang, J. A. Kong, and S. R. T., Theory of Microwave Remote Sensing. New York, USA: John Wiley \& Sons, 1985.

[38] F. T. Ulaby and M. C. Dobson, Handbook of Radar Scattering Statistics for Terrain. Artech House, 1989.

[39] J. C. Curlander and R. N. McDonough, Synthetic Aperture Radar. New York, USA: John Wiley \& Sons, 1991.

[40] C. Oliver and S. Quegan, Understanding Synthtic Aperture Images. Boston, USA: Artech House, 1998.

[41] M. Soumekh, Synthetic Aperture Radar Signal Processing. New York, USA: John Wiley \& Sons, 1999.

[42] I. Cumming and F. H. Wong, Digital Processing of Synthetic Aperture Radar Data. Norwood, USA: Artech House, 2005.

[43] G. Franceschetti and R. Lanari, Synthetic Aperture Radar Processing. Boca Raton, USA: CRC Press, 1999.

[44] G. Carrara, R. S. Goodman, and M. R. M., Spotlight Synthetic Aperture Radar. Norwood, USA: Artech House, 1995.

[45] A. Monti Guarnieri, C. Prati, and F. Rocca, "Interferometry with ScanSAR," in Proceedings of the IEEE Geoscience and Remote Sensing Symposium (IGARSS), (Firenze, ITA), pp. 550-552, Jul. 1995.

[46] F. De Zan and A. Monti Guarnieri, "TOPSAR: Terrain Observation by Progressive Scans," IEEE Transactions on Geoscience and Remote Sensing, vol. 44, pp. 2352 2360, Aug. 2006.

[47] G. Schreier, SAR Geocoding: Data and Systems. Karlsruhe, GER: Wichmann Verlag, 1993. 
[48] S. N. Madsen and H. A. Zebker, Principles \& Applications of Imaging Radar (Manual of Remote Sensing (3rd ed.), vol. 2). New York, USA: John Wiley \& Sons, 1998.

[49] P. A. Rosen, S. Hensley, I. R. Joughin, F. K. Li, S. N. Madsen, E. Rodriguez, and R. M. Goldstein, "Synthetic Aperture Radar Interferometry," Proceedings of IEEE, vol. 88, pp. 333-381, Mar. 2000.

[50] Y. Zhang, "Along Track Interferometry Synthetic Aperture Radar (ATI-SAR) Techniques for Ground Moving Target Detection," Final Technical Report AFRLSN-RS-TR-2005-410, Stiefvater Consultants, 2006.

[51] F. Meyer, S. Hinz, A. Laika, and R. Weihing, D. amd Bamler, "Performance Analysis of the TerraSAR-X Traffic Monitoring Concept," ISPRS Journal of Photogrammetry and Remote Sensing, vol. 61, pp. 225-242, Oct. 2006.

[52] H. A. Zebker and J. Villasenor, "Decorrelation in Interferometric Radar Echoes," IEEE Transactions on Geoscience and Remote Sensing, vol. 30, pp. 950-959, Sep. 1992.

[53] R. Goldstein, H. Zebcker, and C. Werner, "Satellite Radar Interferometry: TwoDimensional Phase Unwrapping," Radioscience, vol. 23, no. 4, pp. 713-720, 1988.

[54] D. Ghiglia and L. Romero, "Robust Two-Dimensional Weighted and Unweighted Phase Unwrapping that Uses Fast Transforms and Iterative Methods," Journal of the Optical Society of America A, vol. 11, no. 1, pp. 107-117, 1994.

[55] M. Costantini, "A Novel Phase Unwrapping Method Based on Network Programming," IEEE Transactions on Geoscience and Remote Sensing, vol. 36, pp. 813821, May 1998.

[56] C. Chen and H. Zebker, "Phase Unwrapping for Large SAR Interferograms: Statistical Segmentation and Generalized Network Models," IEEE Transactions on Geoscience and Remote Sensing, vol. 40, pp. 1709-1719, Nov. 2002.

[57] M. Lachaise, T. Fritz, and M. Eineder, "A New Dual Baseline Phase Unwrapping Algorithm for the tanDEM-X Mission," in Proceedings of the European Conference on Synthetic Aperture Radar (EUSAR), (Friedrichshafen, GER), pp. 1-4, Jun. 2008.

[58] R. Werninghaus and S. Buckreuss, "The TerraSAR-X Mission and System Design," IEEE Transactions on Geoscience and Remote Sensing, vol. 48, pp. 606614, Feb. 2010. 
[59] M. Zink, M. Bachmann, B. Bräutigam, T. Fritz, I. Hajnsek, A. Moreira, B. Wessel, and G. Krieger, "TanDEM-X: The New Global DEM Takes Shape," IEEE Geoscience and Remote Sensing Magazine, vol. 2, pp. 8-23, Jun. 2014.

[60] B. Bräutigam, J. H. Gonzalez, M. Schwerdt, and M. Bachmann, "TerraSAR-X Instrument Calibration Results and Extension for TanDEM-X," IEEE Transactions on Geoscience and Remote Sensing, vol. 48, pp. 702-715, Feb. 2010.

[61] K. Schmidt, G. Castellanos Alfonzo, N. Tous-Ramon, M. Bachmann, and M. Schwerdt, "Calibration Performance of the TerraSAR-X and TanDEM-X Satellites Since Launch," in Proceedings of the European Conference on Synthetic Aperture Radar (EUSAR), (Berlin, GER.), pp. 1164-1167, Jun. 2014.

[62] "Global 30 Arc-Second Elevation (GTOPO30)," Apr. 2018. Available at https: //lta.cr.usgs.gov/GTOPO30/.

[63] A. Moreira, G. Krieger, and J. Mittermayer, "Satellitenkonfiguration zur Interferometrischen und/oder Tomographischen Abbildung der Erdoberfläche Mittels Radar mit Synthetischer Apertur," Jan. 2003. Patent no. EP 1273518 A2.

[64] A. Moreira, G. Krieger, and J. Mittermayer, "Satellite Configuration for Interferometric and/or Tomographic Remote Sensing by Means of Synthetic Aperture Radar (SAR),” Jan. 2003. Patent no. US 6,677,884 B2.

[65] M. Bachmann, B. Bräutigam, D. Schulze, G. Krieger, and M. Zink, “TanDEM-X Acquisition Plan and DEM Performance in the Third Year of Operation," in Proceedings of the Asia-Pacific Conference on Synthetic Aperture Radar (APSAR), (Tsukuba, JP), pp. 12-15, Sep. 2013.

[66] D. Borla Tridon, M. Bachmann, D. Schulze, M. D. Polimeni, M. Martone, J. Böer, and M. Zink, "TanDEM-X DEM Difficult Terrain and Antarctica Acquisitions towards the Planning of the Science Phase," in Proceedings of the European Conference on Synthetic Aperture Radar (EUSAR), (Berlin, GER.), pp. 1133-1136, Jun. 2014.

[67] M. Martone, B. Bräutigam, P. Rizzoli, N. Yague-Martinez, and G. Krieger, "Enhancing Interferometric SAR Performance Over Sandy Areas: Experience From the TanDEM-X Mission," IEEE Journal of Selected Topics in Applied Earth Observations and Remote Sensing, vol. 9, pp. 1036-1046, Mar. 2016.

[68] P. Rizzoli, M. Martone, C. Gonzalez, C. Wecklich, B. Bräutigam, D. Borla Tridon, M. Bachmann, D. Schulze, T. Fritz, M. Huber, B. Wessel, G. Krieger, M. Zink, and A. Moreira, "Generation and Performance Assessment of the Global TanDEM-X 
Digital Elevation Model,' ISPRS Journal of Photogrammetry and Remote Sensing, vol. 132, pp. 119-139, Oct. 2017.

[69] G. Krieger, M. Younis, S. Huber, F. Bordoni, A. Patyuchenko, J. Kim, P. Laskowski, M. Villano, T. Rommel, P. Lopez-Dekker, and A. Moreira, "Digital Beamforming and MIMO SAR: Review and New Concepts," in Proceedings of the European Conference on Synthetic Aperture Radar (EUSAR), (Nuremberg, GER), pp. 11-14, Apr. 2012.

[70] J. L. Bueso-Bello, M. Martone, P. Prats-Iraola, C. Gonzalez-Chamorro, T. Kraus, J. Reimann, M. Jäger, B. Bräutigam, P. Rizzoli, and M. Zink, "Performance Analysis of TanDEM-X Quad-Polarization Products in Pursuit Monostatic Mode," IEEE Journal of Selected Topics in Applied Earth Observations and Remote Sensing, vol. 10, pp. 1853-1869, May 2017.

[71] J. L. Bueso-Bello, P. Prats-Iraola, M. Martone, J. Reimann, U. Steinbrecher, and P. Rizzoli, "Performance Evaluation of TanDEM-X Quad-Polarization Products in Bistatic Mode," IEEE Journal of Selected Topics in Applied Earth Observations and Remote Sensing, vol. 11, pp. 787-799, Mar. 2018.

[72] R. Romeiser, H. Runge, S. Suchandt, R. Kahle, C. Rossi, and P. S. Bell, "Quality Assessment of Surface Current Fields From TerraSAR-X and TanDEM-X AlongTrack Interferometry and Doppler Centroid Analysis," IEEE Transactions on Geoscience and Remote Sensing, vol. 52, pp. 2759-2772, May 2014.

[73] A. Thiele, M. M. Wurth, M. Even, and S. Hinz, "Extraction of Building Shape from TanDEM-X Data," in Proceedings of International Archives of the Photogrammetry, Remote Sensing and Spatial Information Sciences XL-1 W, (Hannover, GER), pp. 345-350, May 2013.

[74] C. Rossi and E. Erten, "Generation of Rice Crops Temporal Change Maps with Differential TanDEM-X Interferometry," in Proceedings of the Geoscience and Remote Sensing Symposium (IGARSS), (Quebec City, CA), pp. 958-961, Jul. 2014.

[75] M. Zink, "TanDEM-X: Operational DEM Generation and Pre-cursor for Future SAR Missions," in Proceedings of the Asia-Pacific Conference on Synthetic Aperture Radar (APSAR), (Tsukuba, JAP), p. 4, Jep. 2013.

[76] T. Sjoegran, V. Vu, and P. Mats, "Experimental Result for SAR GMTI Using Monostatic Pursuit Mode of TerraSAR-X and TanDEM-X on Staring Spotlight Images," in Proceedings of the European Conference on Synthetic Aperture Radar (EUSAR), (Hamburg, GER), pp. 207-2010, Jun. 2016. 
[77] A. Moreira, G. Krieger, I. Hajnsek, K. Papathanassiou, M. Younis, P. LopezDekker, S. Huber, M. Villano, M. Pardini, M. Eineder, F. De Zan, and A. Parizzi, "Tandem-L: A Highly Innovative Bistatic SAR Mission for Global Observation of Dynamic Processes on the Earth's Surface," IEEE Geoscience and Remote Sensing Magazine, vol. 3, pp. 8-23, Jul. 2015.

[78] C. Rossi, F. Rodriguez Gonzalez, T. Fritz, N. Yague-Martinez, and M. Eineder, "TanDEM-X Calibrated Raw DEM Generation," ISPRS Journal of Photogrammetry and Remote Sensing, vol. 73, pp. 12-20, Sep. 2012.

[79] M. Schwerdt, D. Schrank, M. Bachmann, and J. Hueso Gonzalez, "Calibration of the TerraSAR-X and the TanDEM-X Satellite for the TerraSAR-X Mission," in Proceedings of the European Conference on Synthetic Aperture Radar (EUSAR), (Nurenberg, GER), pp. 56-59, Apr. 2012.

[80] T. Fritz and M. Eineder, "TerraSAR-X - Basic Product Specification Document," Public Document TX-GS-DD-3302, German Aerospace Center (DLR), 2009.

[81] M. Martone, P. Rizzoli, B. Bräutigam, and G. Krieger, "Generation of a Global Forest/Non-Forest Map from TanDEM-X Interferometric Data," in Proceedings of the IEEE Geoscience and Remote Sensing Symposium (IGARSS), (Milan, IT), pp. 2634-2637, Jul. 2015.

[82] M. Martone, B. Bräutigam, and G. Krieger, "Quantization Effects in TanDEM-X Data," IEEE Transactions on Geoscience and Remote Sensing, vol. 53, pp. 583597, Feb. 2015.

[83] P. Rizzoli and B. Bräutigam, "Radar Backscatter Modeling Based on Global TanDEM-X Mission Data," IEEE Transactions on Geoscience and Remote Sensing, vol. 52, pp. 5974-5988, Sep. 2014.

[84] J. A. Richards, G.-Q. Sun, and D. S. Simonett, "L-Band Radar Backscatter Modeling of Forest Stands," IEEE Transactions on Geoscience and Remote Sensing, vol. 43, pp. 487-498, Jul. 1987.

[85] G. Sun and K. J. Ranson, "A Three-Dimensional Radar Backscatter Model of Forest Canopies," IEEE Transactions on Geoscience and Remote Sensing, vol. 33, pp. 372-382, Mar. 1995.

[86] J. M. Martinez, N. Floury, T. Le Toan, and A. Beaudoin, "Measurements and Modeling of Vertical Backscatter Distribution in Forest Canopy," IEEE Transactions on Geoscience and Remote Sensing, vol. 38, pp. 710-719, Mar. 2000. 
[87] H. Stephen and D. G. Long, "Microwave Backscatter Modeling of Erg Surfaces in the Sahara Desert," IEEE Transactions on Geoscience and Remote Sensing, vol. 43, pp. 487-498, Feb. 2005.

[88] I. S. Ashcraft and D. G. Long, "Observation and Characterization of Radar Backscatter Over Greenland," IEEE Transactions on Geoscience and Remote Sensing, vol. 43, pp. 225-237, Feb. 2005.

[89] I. Gherboudj, M. Bernier, and R. Leconte, “A Backscatter Modeling for River Ice: Analysis and Numerical Results," IEEE Transactions on Geoscience and Remote Sensing, vol. 48, pp. 1788-1798, Apr. 2010.

[90] G. Parrella, I. Hajnsek, and K. P. Papathanassiou, "Polarimetric Decomposition of L-Band PolSAR Backscattering over the Austfonna Ice Cap," IEEE Transactions on Geoscience and Remote Sensing, vol. 54, pp. 1267-1281, Mar. 2016.

[91] D. Small, N. Miranda, and E. Meier, "A Revised Radiometric Normalization Standard for SAR," in Proceedings of the IEEE Geoscience and Remote Sensing Symposium (IGARSS), (Cape Town, SA), pp. 566-569, Jul. 2009.

[92] R. Raney, T. Freeman, R. Hawkins, and R. Bamler, "A Plea for Radar Brightness," in Proceedings of the IEEE Geoscience and Remote Sensing Symposium (IGARSS), (Pasadena, USA), pp. 1090-1092, Jul. 1994.

[93] T. Fritz and M. Eineder, “TanDEM-X Experimental Product Specifications," Public Document TD-GS-PS-3028, German Aerospace Center (DLR), 2012.

[94] "Extraction of Beta Nought and Sigma Nought from RADARSAT CDPF products," System Report AS97-5001, ALTRIX, 2000.

[95] B. Rosich and P. Meadows, "Absolute Calibration of ASAR Level 1 Products Generated with PF-ASAR," Technical Note ENVI-CLVL-EOPG-TN-03-0010, European Space Agency (ESA), 2004.

[96] M. Bachmann, J. Gonzalez, G. Krieger, M. Schwerdt, J. Antony, and F. De Zan, "Calibration of the Bistatic TanDEM-X Interferometer," in Proceedings of the European Conference on Synthetic Aperture Radar (EUSAR), (Nurenberg, GER), pp. 97-100, Apr. 2012.

[97] "European Space Agency GlobCover Portal," Apr. 2018. Available at http: //due.esrin.esa.int/globcover/.

[98] P. Rizzoli, B. Bräutigam, S. Wollstadt, and J. Mittermayer, "Radar Backscatter Mapping Using TerraSAR-X," IEEE Transactions on Geoscience and Remote Sensing, vol. 49, pp. 3538-3547, Oct. 2011. 
[99] S. Kay, Fundamentals of Statistical Signal Processing: Estimation Theory. Upper Saddle River, New Jersey, USA: Prentice Hall PTR, 1993.

[100] P. C. Dubois, J. van Zyl, and T. Engman, "Measuring Soil Moisture with Imaging Radars," IEEE Transactions on Geoscience and Remote Sensing, vol. 33, pp. 915926, Jul. 1995.

[101] J. Alvarez-Perez, M. Schwerdt, and M. Bachmann, "Antenna Elevation Pattern Estimation from Rain Forest Acquisitions," in Proceedings of the Geoscience and Remote Sensing Symposium (IGARSS), (Denver, USA), pp. 3857-3860, Aug. 2006.

[102] A. Danklmayer, B. Döring, M. Schwerdt, and M. Chandra, "Assessment of Atmospheric Propagation Effects in SAR Images," IEEE Transactions on Geoscience and Remote Sensing, vol. 47, pp. 3507-3518, Oct. 2009.

[103] C. S. Benson, "Stratigraphic studies in the snow and firn of the greenland ice sheet," Research Report 70, U.S. Army Snow, Ice and Permafrost Research Establishment, 1962 (Reprinted Aug. 1996).

[104] T. G. Farr, P. A. Rosen, E. Caro, R. Crippen, R. Duren, S. Hensley, M. Kobrick, M. Paller, E. Rodriguez, L. Roth, D. Seal, S. Shaffer, J. Shimada, J. Umland, M. Werner, M. Oskin, D. Burbank, and D. Alsdorf, "The Shuttle Radar Topography Mission," Reviews of Geophysics, vol. 45, no. 3/4, pp. 1-33, 2007.

[105] M. Martone, P. Rizzoli, B. Bräutigam, and G. Krieger, "First Two Years of TanDEM-X Mission: Interferometric Performance Overview," Radio Science, vol. 48, pp. 617-627, Sep. 2013.

[106] M. Shimada and A. Freeman, "A Technique for Measurement of Spaceborne SAR Antenna Patterns using Distributed Targets," IEEE Transactions on Geoscience and Remote Sensing, vol. 33, pp. 100-114, Jan. 1995.

[107] Z. Bartalis, W. Wagner, V. Naeimi, D. Sabel, and C. Pathe, "SAR Calibration Requirements for Soil Moisture Estimation," in Proceedings of the CEOS SAR Calibration and Validation Workshop, (Pasadena, USA), Nov. 2009.

[108] S. Cote, S. Srivastava, S. Muir, and T. Lukowski, "Assessment of Distributed Target Sites within the Radarsat program," in Proceedings of the CEOS SAR Calibration and Validation Workshop, (Fairbanks, USA), Nov. 2011.

[109] H. Woodhouse, J. van der Sander, and D. Hoekman, "Scatterometer Observations of Seasonal Backscatter Variation Over Tropical Rain Forest," IEEE Transactions on Geoscience and Remote Sensing, vol. 37, pp. 859-861, Mar. 1999. 
[110] D. G. Long and G. B. Skouson, "Calibration of Spaceborne Scatterometers Using Tropical Rain Forests," IEEE Transactions on Geoscience and Remote Sensing, vol. 34, pp. 413-424, Mar. 1996.

[111] P. Rizzoli, B. Bräutigam, and M. Zink, "TanDEM-X Large-Scale Study of Tropical Rainforests for Spaceborne SAR Calibration in X-band," in Proceedings of the European Conference on Synthetic Aperture Radar (EUSAR), (Berlin, GER), pp. 1168-1171, Jun. 2014.

[112] P. Rizzoli, M. Martone, H. Rott, and A. Moreira, "Characterization of Snow Facies on the Greenland Ice Sheet Observed by TanDEM-X Interferometric SAR Data," Remote Sensing, vol. 9, Mar. 2017.

[113] A. Groh, H. Ewert, M. Fritsche, A. Rülke, R. Rosenau, M. Scheinert, and R. Dietrich, "Assessing the Current Evolution of the Greenland Ice Sheet by Means of Satellite and Ground-Based Observations," Surveys in Geophysics, vol. 35, pp. 1459-1480, Nov. 2014.

[114] R. Bales, Q. Guo, D. Shen, J. McConnell, G. Du, and J. Burkhart, "Annual Accumulation for Greenland Updated Using Ice Core Data Developed During 2000/2006 and Analysis of Daily Coastal Meteorological Data," Journal of Geophysical Research, vol. 114, Mar. 2009.

[115] J. Munk, K. C. Jezek, R. R. Foster, and S. P. Gogineni, "An Accumulation Map for the Greenland Dry-Snow Facies Derived from Spaceborne Radar," Journal of Geophysical Research, vol. 108, May 2003.

[116] K. G. Jezek, P. Gogineni, and M. Shanableh, "Radar Mesurements of Melt Zones on the Greenland Ice Sheet," Geophisical Research Letters, vol. 21, pp. 33-36, Jan. 1994.

[117] E. Rignot, K. Jezek, J. van Zyl, and M. Drinkwater, "Radar Scattering from Snow Facies of the Greenland Ice Sheet: Results from the AIRSAR 1991 Campaign," in Proceedings of the IEEE Geoscience and Remote Sensing Symposium (IGARSS), vol. 3, (Tokyo, JP), pp. 1270-1272, Aug. 1993.

[118] I. Joughin, B. E. Smith, I. M. Howat, T. Moon, and T. A. Scambos, "A SAR Record of Early 21st Century Change in Greenland," Journal of Glaciology, vol. 62, pp. 62-71, Feb. 2016.

[119] E. Weber Hoen and H. Zebker, "Penetration Depths Inferred from Interferometric Volume Decorrelation Observed over the Greenland Ice Sheet," IEEE Transactions on Geoscience and Remote Sensing, vol. 38, pp. 2571-2583, Nov. 2000. 
[120] H. Rott, K. Sturm, and H. Miller, "Active and Passive Microwave Signatures of Antarctic Firn by Means of Field Measurements and Satellite Data," Annals of Galciology, vol. 17, pp. 337-343, 1993.

[121] H. Rott, D. Floricioiu, J. Wuite, S. Scheiblauer, T. Nagler, and M. Kern, "Mass Changes of Outlet Glaciers Along the Nordensjköld Coast, Northern Antarctic Peninsula, based on TanDEM-X Satellite Measurements," Geophisical Research Letter, vol. 41, pp. 8123-8129, 2014.

[122] J. Munk, K. Jezek, F. Baumgartner, S. P. Gogineni, R. R. Forster, and I. H. H. Zabel, "Phase Unwrapping Correction with Dual-Baseline Data for the TanDEM$\mathrm{X}$ Mission," in Proceedings of the IEEE International Geoscience and Remote Sensing Symposium (IGARSS), (Honolulu, USA), July 2000.

[123] P. Rizzoli, M. Martone, and B. Bräutigam, "Greenland Snow Facies Identification Approach using TanDEM-X Interferometric Data," in Proceedings of the IEEE Geoscience and Remote Sensing Symposium (IGARSS), (Milan, IT), pp. 20602063, Jul. 2015.

[124] J. Bezdek, R. Ehrlich, and W. Full, "FCM: The Fuzzy c-Means Clustering Algorithm,” Computers and Geosciences, vol. 10, no. 2-3, pp. 191-203, 1984.

[125] K. C. Partington, "Discrimination of Glacier Facies using Multi-Temporal SAR Data," Journal of Glaciology, vol. 44, no. 146, pp. 42-53, 1998.

[126] N. Tran, F. Rémy, H. Feng, and P. Féménias, "Snow Facies Over Ice Sheets Derived From Envisat Active and Passive Observations," IEEE Transactions on Geoscience and Remote Sensing, vol. 46, pp. 3694-3708, Nov. 2008.

[127] F. Höppner, F. Klawonn, R. Kruse, and T. Runkler, Fuzzy Cluster Analysis: Methods for Classification, Data Analysis and Image Recognition. New York, USA: John Wiley \& Sons, 1999.

[128] S. Miyamoto, H. Ichihashi, and K. Honda, Algorithms for Fuzzy Clustering: Methods in c-Means Clustering with Applications. New York, USA: Springer Science \& Business Media, 2008.

[129] R. A. Derrig and K. M. Ostaszewski, "Fuzzy Techniques of Pattern Recognition in Risk and Claim Classification," Journal of Risk and Insurance, vol. 62, no. 3, pp. 447-482, 1995.

[130] S. Das and H. K. Baruah, "Application of Fuzzy c-Means Clustering Technique in Vehicular Pollution," Journal of Process Management New Technologies, vol. 1, no. 3, pp. 96-107, 2013. 
[131] M. Goyal and S. Kumar, "Improving the Initial Centroids of k-Means Clustering Algorithm to Generalize its Applicability," Journal of The Institution of Engineers (India): Series B, vol. 95, pp. 345-350, Dec. 2014.

[132] P. Rizzoli, M. Martone, and B. Bräutigam, "Global Interferometric Coherence Maps from TanDEM-X Quicklook Data," IEEE Geoscience and Remote Sensing Letters, vol. 11, pp. 1861-1865, November 2014.

[133] M. Citterio and A. Ahlstrom, "The Aerophotogrammetric Map of Greenland Ice Masses," The Cryosphere, vol. 7, pp. 445-449, Mar. 2013.

[134] M. Tedesco, "Greenland Daily Surface Melt 25km EASE-Grid, [Data from 1979 to 2010]," digital media, City College of New York, The City University of New York, 2010.

[135] S. V. Nghiem, D. K. Hall, T. L. Mote, M. Tedesco, M. R. Albert, K. Keegan, C. A. Shuman, N. E. DiGirolamo, and G. Neumann, "The Extreme Melt Across the Greenland Ice Sheet in 2012," Geophysical Research Letters, vol. 39, pp. 1-6, Oct. 2012.

[136] J. Nilsson, P. Vallelonga, S. B. Simonsen, L. Sandberg Sørensen, R. Forsberg, D. Dahl-Jensen, M. Hirabayashi, K. Goto-Azuma, C. S. Hvidberg, H. A. Kjær, and K. Satow, "Greenland 2012 Melt Event Effects on CryoSat-2 Radar Altimetry," Geophysical Research Letters, vol. 42, pp. 3919-3926, May 2015.

[137] ASCAT, "Advanced scatterometer (ASCAT) Sigma-0 browse images (nonenhanced)," Apr. 2018. Available at http://www.scp.byu.edu/data/ Ascat/SigBrw/ASCAT_SigBrw.html.

[138] "The International Glaciological Expedition to Greenland (EGIG), 1956-74," Polar Record 110, 1975.

[139] D. J. Wingham, C. R. Francis, S. Baker, C. Bouzinac, D. Brockley, R. Cullen, P. de Chateau-Thierry, S. W. Laxon, U. Mallow, C. Mavrocordatos, L. Phalippou, G. Ratier, L. Rey, F. Rostan, P. Viau, and D. W. Wallis, "CryoSat: A Mission to Determine Fluctuations in the Earth's Ice Fields," Advances in Space Research, vol. 37, pp. 841-871, Oct. 2006.

[140] J. B. T. Scott, P. Nienow, D. Mair, V. Parry, E. Morris, and D. J. Wingham, "Importance of Seasonal and Annual Layers in Controlling Backscatter to Radar Altimeter Across the Percolation Zone of an Ice Sheet," Geophisical Reseach Letters, vol. 33, Dec. 2006. 
[141] E. M. Morris and D. J. Wingham, "The Effect of Fluctuations in Surface Density, Accumulation and Compaction on Elevation Changes along the EGIG Line, Central Greenland," Journal of Glaciology, vol. 57, no. 203, pp. 416-430, 2011.

[142] J. B. T. Scott, D. Mair, P. Nienow, V. Parry, and E. M. Morris, "A Ground-Based Radar Backscatter Investigation in the Percolation Zone of the Greenland Ice Sheet," Remote Sensing of Environment, vol. 104, pp. 361-373, May 2006.

[143] J. Flach, K. Partington, C. Ruiz, E. Jeansou, and M. Drinkwater, "Inversion of the surface properties of ice sheets from satellite microwave data," IEEE Transactions on Geoscience and Remote Sensing, vol. 43, pp. 743-752, Apr. 2005.

[144] M. Martone, P. Rizzoli, and G. Krieger, "Volume Decorrelation Effects in TanDEM-X Interferometric Data," IEEE Geoscience and Remote Sensing Letters, pp. 1812-1816, 2016.

[145] S. Fujita, M. Hirabayashi, K. Goto-Azuma, R. Dallmayr, K. Satow, J. Zheng, and D. Dahl-Jensen, "Densification of Layered Firn of the Ice Sheet at NEEM, Greenland," Journal of Glaciology, vol. 60, pp. 905-921, Nov. 2014.

[146] ICESat, "GLAS/ICESat L2 Global Land Surface Altimetry Data, Version 34. [Subset GLA14, Release 31]," Apr. 2018. Available at http: / / dx . doi . org / 10.5067 / ICESAT/GLAS / DATA227.

[147] J. Dall, "InSAR Elevation Bias Caused by Penetration Into Uniform Volumes," IEEE Transactions on Geoscience and Remote Sensing, vol. 45, pp. 2319-2324, Jul. 2007.

[148] B. Wessel, A. Bertram, A. Gruber, S. Bemm, and S. Dech, "A New HighResolution Elevation Model of Greenland Derived from TanDEM-X," in ISPRS Annals of Photogrammetry, Remote Sensing, and Spatial Information Sciences, pp. 9-16, July 2016.

[149] D. Sabel, M. Doubkova, W. Wagner, and Z. Bartalis, "Sigma Nought Statistics over Land Activitiy, ESA contract No. 22122/08/NL/JA," final report, Institute of Photogrammetry and Remote Sensing, Vienna University of Technology, Austria, 2009.

[150] S. Haykin, "Cognitive Radar: A Way of the Future," IEEE Signal Processing Magazine, vol. 23, pp. 30-40, Feb. 2006.

[151] M. Martone, B. Bräutigam, and G. Krieger, "Azimuth-Switched Quantization for SAR Systems and Performance Analysis on TanDEM-X Data," IEEE Geoscience and Remote Sensing Letters, vol. 11, pp. 181-185, Jan. 2014. 
[152] J. Bueso-Bello, P. Rizzoli, M. Martone, and C. Gonzalez, "Potentials of TanDEM$\mathrm{X}$ Forest/Non-Forest Map for Change Detection," in Proceedings of the Geoscience and Remote Sensing Symposium (IGARSS), 2018, Accepted Paper. 
Appendices 


\section{A. Backscatter Models Database}

In this appendix, the reader can find a summary of backscatter statistics and mean $\beta^{0}$ fitting coefficients. For each GlobCover class, a table is provided, where all parameters were derived using data acquired over one single continent only. The continent' choice was based on both the number of available observations and on how representative such a continent is for the considered ground cover class. Moreover, backscatter statistics and corresponding fitting coefficients are always presented for two different seasons: winter and summer. Intermediate seasons (spring and autumn) are here omitted, since their definition can be rather subjective and the risk of mixing together very different conditions is quite high.

Each table displays the following quantities:

- $\mathrm{E}[\theta]$ : mean incidence angle for the considered interval,

- Nsamples: number of available input observations,

- $\mathrm{E}\left[\beta^{0}\right]$ : mean radar brightness $\beta^{0}$,

- $\sigma_{\beta^{0}}: \beta^{0}$ standard deviation,

- $\mathrm{E}\left[\beta^{0}\right]_{w}$ : weighted mean $\beta^{0}$,

- $\left[c_{0}, c_{1}, c_{2}, c_{3}\right]$ : polynomial fitting coefficients (derived from the weighted $\beta^{0}$ mean value).

The incident-dependent polynomial models in $\mathrm{dB}$ of the weighted mean radar brightness $\beta_{W}^{0}(\theta)$ can then be derived according to (5.25) as:

$$
\beta_{W}^{0}(\theta)=c_{0}+c_{1} \theta+c_{2} \theta^{2}+c_{3} \theta^{3},
$$

where the incidence angle $\theta$ is expressed in degrees. 


\begin{tabular}{|c|c|c|c|c|c|c|c|c|c|c|c|}
\hline \multirow{2}{*}{\multicolumn{2}{|c|}{$\begin{array}{c}\text { Class } 1 \\
\text { Continent }\end{array}$}} & \multicolumn{10}{|c|}{ Post-flooding or irrigated croplands (or aquatic) } \\
\hline & & \multicolumn{10}{|c|}{ Asia } \\
\hline \multicolumn{2}{|c|}{ Season } & \multicolumn{5}{|c|}{ Winter } & \multicolumn{5}{|c|}{ Summer } \\
\hline \multirow[t]{2}{*}{ Interval } & $\mathrm{E}[\theta]$ & Nsamples & $\mathrm{E}\left[\beta^{0}\right]$ & $\mathrm{E}\left[\beta^{0}\right]-\sigma_{\beta^{0}}$ & $\mathrm{E}\left[\beta^{0}\right]+\sigma_{\beta^{0}}$ & $\mathrm{E}\left[\beta^{0}\right]_{w}$ & Nsamples & $\mathrm{E}\left[\beta^{0}\right]$ & $\mathrm{E}\left[\beta^{0}\right]-\sigma_{\beta^{0}}$ & $\mathrm{E}\left[\beta^{0}\right]+\sigma_{\beta^{0}}$ & $\mathrm{E}\left[\beta^{0}\right]_{w}$ \\
\hline & {$\left[{ }^{\circ}\right]$} & & {$[\mathrm{dB}]$} & {$[\mathrm{dB}]$} & {$[\mathrm{dB}]$} & {$[\mathrm{dB}]$} & & {$[\mathrm{dB}]$} & {$[\mathrm{dB}]$} & {$[\mathrm{dB}]$} & {$[\mathrm{dB}]$} \\
\hline 1 & 25 & 0 & & & & & 81507 & -3.11 & -5.20 & -1.71 & -2.96 \\
\hline 2 & 27 & 1056 & -3.40 & -5.27 & -2.10 & -3.40 & 11323984 & -3.50 & -6.41 & -1.77 & -3.54 \\
\hline 3 & 29 & 63930560 & -5.14 & -8.61 & -3.23 & -4.95 & 17601344 & -4.68 & -8.21 & -2.76 & -3.81 \\
\hline 4 & 31 & $2.1825242 \mathrm{e}+08$ & -5.53 & -9.39 & -3.52 & -5.07 & 13258009 & -5.02 & -8.96 & -2.99 & -4.20 \\
\hline 5 & 33 & $3.7805651 \mathrm{e}+08$ & -6.20 & -10.14 & -4.17 & -5.56 & 31495209 & -5.70 & -9.62 & -3.67 & -4.65 \\
\hline 6 & 35 & $3.4571636 \mathrm{e}+08$ & -6.94 & -11.05 & -4.87 & -6.22 & 48110609 & -6.51 & -10.50 & -4.47 & -5.34 \\
\hline 7 & 37 & $1.8439901 \mathrm{e}+08$ & -7.42 & -11.29 & -5.41 & -6.80 & $1.6815604 \mathrm{e}+08$ & -7.30 & -10.44 & -5.50 & -5.60 \\
\hline 8 & 39 & $1.6885608 \mathrm{e}+08$ & -7.82 & -11.98 & -5.73 & -7.04 & $4.6601079 \mathrm{e}+08$ & -7.16 & -10.56 & -5.28 & -5.86 \\
\hline 9 & 41 & $1.6448995 \mathrm{e}+08$ & -8.41 & -12.65 & -6.31 & -7.64 & $6.5617965 \mathrm{e}+08$ & -7.60 & -10.95 & -5.73 & -6.62 \\
\hline 10 & 43 & $1.6344105 \mathrm{e}+08$ & -8.82 & -12.95 & -6.74 & -8.07 & $4.2592170 \mathrm{e}+09$ & -7.01 & -10.11 & -5.22 & -5.51 \\
\hline 11 & 45 & $2.0852802 \mathrm{e}+08$ & -9.57 & -14.00 & -7.42 & -9.03 & $5.8462554 \mathrm{e}+08$ & -8.56 & -12.21 & -6.60 & -8.06 \\
\hline 12 & 47 & $2.9207566 \mathrm{e}+08$ & -9.63 & -13.94 & -7.51 & -9.26 & $2.4299079 \mathrm{e}+08$ & -8.66 & -12.43 & -6.67 & -8.19 \\
\hline \multirow[t]{5}{*}{13} & 49 & 76022474 & -9.21 & -13.03 & -7.21 & -8.82 & 16649384 & -8.57 & -12.56 & -6.53 & -7.39 \\
\hline & & & & \multicolumn{8}{|c|}{ "Fitting Coefficients } \\
\hline & & & & \multicolumn{4}{|c|}{ Winter } & \multicolumn{4}{|c|}{ Summer } \\
\hline & & & & $c_{0}$ & $c_{1}$ & $c_{2}$ & $c_{3}$ & $c_{0}$ & $c_{1}$ & $c_{2}$ & $c_{3}$ \\
\hline & & & & 17.298037 & -70.054636 & 66.002779 & -23.800131 & -3.7294054 & 19.694896 & -54.774542 & 30.631802 \\
\hline
\end{tabular}

Table A.1.: Class 1 - Post-flooding or irrigated croplands (or aquatic). Summary of backscatter statistics and fitting coefficients.

\begin{tabular}{|c|c|c|c|c|c|c|c|c|c|c|c|}
\hline \multirow{3}{*}{\multicolumn{2}{|c|}{$\begin{array}{c}\text { Class 2 } \\
\text { Continent } \\
\text { Season }\end{array}$}} & \multicolumn{10}{|c|}{ Rainfed croplands } \\
\hline & & \multicolumn{10}{|c|}{ Europe } \\
\hline & & \multicolumn{5}{|c|}{ Winter } & \multicolumn{5}{|c|}{ Summer } \\
\hline \multirow[t]{2}{*}{ Interval } & $\mathrm{E}[\theta]$ & Nsamples & $\mathrm{E}\left[\beta^{0}\right]$ & $\mathrm{E}\left[\beta^{0}\right]-\sigma_{\beta^{0}}$ & $\mathrm{E}\left[\beta^{0}\right]+\sigma_{\beta^{0}}$ & $\mathrm{E}\left[\beta^{0}\right]_{w}$ & Nsamples & $\mathrm{E}\left[\beta^{0}\right]$ & $\mathrm{E}\left[\beta^{0}\right]-\sigma_{\beta^{0}}$ & $\mathrm{E}\left[\beta^{0}\right]+\sigma_{\beta^{0}}$ & $\mathrm{E}\left[\beta^{0}\right]_{w}$ \\
\hline & {$\left[{ }^{\circ}\right]$} & & {$[\mathrm{dB}]$} & {$[\mathrm{dB}]$} & {$[\mathrm{dB}]$} & {$[\mathrm{dB}]$} & & {$[\mathrm{dB}]$} & {$[\mathrm{dB}]$} & {$[\mathrm{dB}]$} & {$[\mathrm{dB}]$} \\
\hline 1 & 25 & 0 & & & & & 0 & & & & \\
\hline 2 & 27 & 0 & & & & & 0 & & & & \\
\hline 3 & 29 & 0 & & & & & 0 & & & & \\
\hline 4 & 31 & 62906 & -3.37 & -8.92 & -1.02 & -3.54 & 644109 & -5.64 & -10.25 & -3.46 & -5.64 \\
\hline 5 & 33 & 56084225 & -5.84 & -9.94 & -3.77 & -5.61 & 15408860 & -6.12 & -10.80 & -3.93 & -6.09 \\
\hline 6 & 35 & $1.9601733 \mathrm{e}+08$ & -6.47 & -10.81 & -4.35 & -6.05 & 87397705 & -7.04 & -11.31 & -4.93 & -6.75 \\
\hline 7 & 37 & $1.5906569 \mathrm{e}+08$ & -6.96 & -11.25 & -4.84 & -6.66 & $1.1354021 \mathrm{e}+08$ & -7.37 & -11.46 & -5.31 & -6.79 \\
\hline 8 & 39 & $1.1815108 \mathrm{e}+08$ & -7.43 & -12.55 & -5.14 & -7.32 & $2.5331717 \mathrm{e}+08$ & -7.45 & -11.76 & -5.32 & -6.98 \\
\hline 9 & 41 & 90919310 & -7.99 & -13.55 & -5.63 & -7.76 & $4.0926941 \mathrm{e}+08$ & -7.98 & -12.66 & -5.78 & -7.66 \\
\hline 10 & 43 & $1.3145341 \mathrm{e}+08$ & -9.05 & -13.78 & -6.84 & -9.46 & $4.2511835 \mathrm{e}+08$ & -8.29 & -13.11 & -6.06 & -8.14 \\
\hline 11 & 45 & $2.7940661 \mathrm{e}+08$ & -9.67 & -14.43 & -7.46 & -10.36 & $3.1413546 \mathrm{e}+08$ & -8.71 & -13.66 & -6.45 & -8.72 \\
\hline 12 & 47 & $1.7629555 \mathrm{e}+08$ & -9.92 & -15.16 & -7.62 & -10.72 & 77652624 & -8.93 & -13.83 & -6.69 & -8.85 \\
\hline \multirow[t]{5}{*}{13} & 49 & 1851964 & -10.34 & -14.78 & -8.19 & -10.73 & 332655 & -9.35 & -15.78 & -6.86 & -9.56 \\
\hline & & & & \multicolumn{8}{|c|}{ Fitting Coefficients } \\
\hline & & & & \multicolumn{4}{|c|}{ Winter } & \multicolumn{4}{|c|}{ Summer } \\
\hline & & & & $c_{0}$ & $c_{1}$ & $c_{2}$ & $c_{3}$ & $c_{0}$ & $c_{1}$ & $c_{2}$ & $c_{3}$ \\
\hline & & & & 53.686524 & -202.83424 & 231.57281 & -96.971102 & 17.156617 & -88.401151 & 117.44627 & -59.182202 \\
\hline
\end{tabular}

Table A.2.: Class 2 - Rainfed croplands. Summary of backscatter statistics and fitting coefficients. 


\begin{tabular}{|c|c|c|c|c|c|c|c|c|c|c|c|}
\hline \multirow{2}{*}{\multicolumn{2}{|c|}{$\begin{array}{c}\text { Class } 3 \\
\text { Continent } \\
\end{array}$}} & \multicolumn{10}{|c|}{ Mosaic cropland (50-70\%) / vegetation (grassland/shrubland/forest) $(20-50 \%)$} \\
\hline & & \multicolumn{10}{|c|}{ Europe } \\
\hline \multicolumn{2}{|c|}{ Season } & \multicolumn{5}{|c|}{ Winter } & \multicolumn{5}{|c|}{ Summer } \\
\hline Interval & $\mathrm{E}[\theta]$ & Nsamples & $\mathrm{E}\left[\beta^{0}\right]$ & $\mathrm{E}\left[\beta^{0}\right]-\sigma_{\beta^{0}}$ & $\mathrm{E}\left[\beta^{0}\right]+\sigma_{\beta^{0}}$ & $\mathrm{E}\left[\beta^{0}\right]_{w}$ & Nsamples & $\mathrm{E}\left[\beta^{0}\right]$ & $\mathrm{E}\left[\beta^{0}\right]-\sigma_{\beta^{0}}$ & $\mathrm{E}\left[\beta^{0}\right]+\sigma_{\beta^{0}}$ & $\mathrm{E}\left[\beta^{0}\right]_{w}$ \\
\hline & {$\left[{ }^{\circ}\right]$} & & {$[\mathrm{dB}]$} & {$[\mathrm{dB}]$} & {$[\mathrm{dB}]$} & {$[\mathrm{dB}]$} & & [dB] & {$[\mathrm{dB}]$} & {$[\mathrm{dB}]$} & {$[\mathrm{dB}]$} \\
\hline 1 & 25 & 0 & & & & & 0 & & & & \\
\hline 2 & 27 & 0 & & & & & 0 & & & & \\
\hline 3 & 29 & 0 & & & & & 0 & & & & \\
\hline 4 & 31 & 302435 & -4.35 & -10.06 & -1.96 & -4.98 & 1609483 & -5.88 & -9.92 & -3.83 & -5.92 \\
\hline 5 & 33 & 74936391 & -5.91 & -10.59 & -3.71 & -5.77 & 12818542 & -5.87 & -10.74 & -3.63 & -5.81 \\
\hline 6 & 35 & $2.2745115 \mathrm{e}+08$ & -6.50 & -11.39 & -4.25 & -6.23 & 66880771 & -7.14 & -11.61 & -4.99 & -6.61 \\
\hline 7 & 37 & $1.8678561 \mathrm{e}+08$ & -6.96 & -11.71 & -4.75 & -6.78 & 98465109 & -7.41 & -12.01 & -5.23 & -6.82 \\
\hline 8 & 39 & $1.4693410 \mathrm{e}+08$ & -7.41 & -12.66 & -5.10 & -7.29 & $2.0075873 e+08$ & -7.53 & -12.25 & -5.32 & -7.09 \\
\hline 9 & 41 & $1.2709042 \mathrm{e}+08$ & -8.14 & -13.54 & -5.81 & -7.83 & $3.5249681 \mathrm{e}+08$ & -8.04 & -13.04 & -5.78 & -7.70 \\
\hline 10 & 43 & $1.7141066 \mathrm{e}+08$ & -8.81 & -13.89 & -6.53 & -8.91 & $3.6434670 \mathrm{e}+08$ & -8.24 & -13.49 & -5.93 & -8.07 \\
\hline 11 & 45 & $3.6927169 \mathrm{e}+08$ & -9.34 & -14.47 & -7.05 & -10.13 & $2.7502116 \mathrm{e}+08$ & -8.63 & -13.95 & -6.31 & -8.57 \\
\hline 12 & 47 & $1.8939200 \mathrm{e}+08$ & -9.62 & -15.01 & -7.29 & -10.44 & 90630675 & -8.89 & -13.99 & -6.61 & -8.78 \\
\hline \multirow[t]{5}{*}{13} & 49 & 1971234 & -10.36 & -15.09 & -8.14 & -10.44 & 2097791 & -9.53 & -14.49 & -7.28 & -9.43 \\
\hline & & & & \multicolumn{8}{|c|}{ Fitting Coefficients } \\
\hline & & & & \multicolumn{4}{|c|}{ Winter } & \multicolumn{4}{|c|}{ Summer } \\
\hline & & & & $c_{0}$ & $c_{1}$ & $c_{2}$ & $c_{3}$ & $c_{0}$ & $c_{1}$ & $c_{2}$ & $c_{3}$ \\
\hline & & & & -42.171168 & 190.18312 & -302.98657 & 144.63935 & -21.365099 & 79.302782 & -123.27955 & 54.909467 \\
\hline
\end{tabular}

Table A.3.: Class 3 - Mosaic cropland (50-70\%) / vegetation (grassland/shrubland/forest) (20-50\%). Summary of backscatter statistics and fitting coefficients.

\begin{tabular}{|c|c|c|c|c|c|c|c|c|c|c|c|}
\hline \multirow{3}{*}{\multicolumn{2}{|c|}{$\begin{array}{c}\text { Class } 4 \\
\text { Continent } \\
\text { Season }\end{array}$}} & \multicolumn{10}{|c|}{ Mosaic vegetation (grassland/shrubland/forest) $(50-70 \%)$ / cropland $(20-50 \%)$} \\
\hline & & \multicolumn{10}{|c|}{ North America } \\
\hline & & \multicolumn{5}{|c|}{ Winter } & \multicolumn{5}{|c|}{ Summer } \\
\hline Interval & $\mathrm{E}[\theta]$ & Nsamples & $\mathrm{E}\left[\beta^{0}\right]$ & $\mathrm{E}\left[\beta^{0}\right]-\sigma_{\beta^{0}}$ & $\mathrm{E}\left[\beta^{0}\right]+\sigma_{\beta^{0}}$ & $\mathrm{E}\left[\beta^{0}\right]_{w}$ & Nsamples & $\mathrm{E}\left[\beta^{0}\right]$ & $\mathrm{E}\left[\beta^{0}\right]-\sigma_{\beta^{0}}$ & $\mathrm{E}\left[\beta^{0}\right]+\sigma_{\beta^{0}}$ & $\mathrm{E}\left[\beta^{0}\right]_{w}$ \\
\hline & {$\left[{ }^{\circ}\right]$} & & {$[\mathrm{dB}]$} & {$[\mathrm{dB}]$} & {$[\mathrm{dB}]$} & {$[\mathrm{dB}]$} & & {$[\mathrm{dB}]$} & {$[\mathrm{dB}]$} & {$[\mathrm{dB}]$} & {$[\mathrm{dB}]$} \\
\hline 1 & 25 & 0 & & & & & 0 & & & & \\
\hline 2 & 27 & 186727 & -2.97 & -5.97 & -1.21 & -3.36 & 360192 & -2.49 & -7.01 & -0.32 & -2.27 \\
\hline 3 & 29 & 24061026 & -4.84 & -8.25 & -2.96 & -4.46 & 2438327 & -3.72 & -8.07 & -1.58 & -3.37 \\
\hline 4 & 31 & 79232894 & -5.76 & -9.28 & -3.84 & -5.58 & 5049529 & -4.64 & -8.31 & -2.68 & -4.38 \\
\hline 5 & 33 & $1.6456802 \mathrm{e}+08$ & -6.83 & -10.28 & -4.93 & -6.92 & 14687500 & -6.48 & -10.09 & -4.54 & -5.85 \\
\hline 6 & 35 & $2.2049142 \mathrm{e}+08$ & -7.49 & -11.01 & -5.57 & -8.61 & 48117607 & -7.29 & -10.53 & -5.46 & -6.90 \\
\hline 7 & 37 & $1.4711806 \mathrm{e}+08$ & -7.74 & -11.11 & -5.86 & -8.23 & $1.5675644 \mathrm{e}+08$ & -7.56 & -10.68 & -5.77 & -7.40 \\
\hline 8 & 39 & $1.0640991 \mathrm{e}+08$ & -7.79 & -11.44 & -5.83 & -8.00 & $3.5658392 \mathrm{e}+08$ & -7.74 & -10.95 & -5.91 & -7.74 \\
\hline 9 & 41 & $1.0186686 \mathrm{e}+08$ & -8.38 & -12.13 & -6.40 & -8.43 & $4.8473614 \mathrm{e}+08$ & -8.31 & -11.59 & -6.46 & -8.52 \\
\hline 10 & 43 & $1.0430749 \mathrm{e}+08$ & -8.64 & -12.17 & -6.72 & -8.82 & $5.3015857 \mathrm{e}+08$ & -8.69 & -11.93 & -6.86 & -9.14 \\
\hline 11 & 45 & $1.4987955 \mathrm{e}+08$ & -9.19 & -12.62 & -7.29 & -9.54 & $4.2211154 \mathrm{e}+08$ & -9.17 & -12.34 & -7.35 & -9.63 \\
\hline 12 & 47 & $1.5143345 \mathrm{e}+08$ & -9.44 & -12.90 & -7.54 & -10.33 & $2.4313097 \mathrm{e}+08$ & -9.36 & -12.51 & -7.56 & -10.22 \\
\hline \multirow[t]{5}{*}{13} & 49 & 17939113 & -9.19 & -12.53 & -7.33 & -10.77 & 12189118 & -8.36 & -11.45 & -6.57 & -8.14 \\
\hline & & & & \multicolumn{8}{|c|}{ Fitting Coefficients } \\
\hline & & & & \multicolumn{4}{|c|}{ Winter } & \multicolumn{4}{|c|}{ Summer } \\
\hline & & & & $c_{0}$ & $c_{1}$ & $c_{2}$ & $c_{3}$ & $c_{0}$ & $c_{1}$ & $c_{2}$ & $c_{3}$ \\
\hline & & & & 86.278627 & -385.61568 & 534.29826 & -252.79241 & -8.2753908 & 90.604967 & -237.49018 & 153.26051 \\
\hline
\end{tabular}

Table A.4.: Class 4 - Mosaic vegetation (grassland/shrubland/forest) (50-70\%) / cropland (20-50\%). Summary of backscatter statistics and fitting coefficients. 


\begin{tabular}{|c|c|c|c|c|c|c|c|c|c|c|c|}
\hline \multirow{3}{*}{\multicolumn{2}{|c|}{$\begin{array}{c}\text { Class } 5 \\
\text { Continent } \\
\text { Season }\end{array}$}} & \multicolumn{10}{|c|}{ Closed to open (>15\%) broadleaved evergreen or semi-deciduous forest $(>5 \mathrm{~m})$} \\
\hline & & \multicolumn{10}{|c|}{ South America } \\
\hline & & \multicolumn{5}{|c|}{ Winter } & \multicolumn{5}{|c|}{ Summer } \\
\hline \multirow[t]{2}{*}{ Interval } & $\mathrm{E}[\theta]$ & Nsamples & $\mathrm{E}\left[\beta^{0}\right]$ & $\mathrm{E}\left[\beta^{0}\right]-\sigma_{\beta^{0}}$ & $\mathrm{E}\left[\beta^{0}\right]+\sigma_{\beta^{0}}$ & $\mathrm{E}\left[\beta^{0}\right]_{w}$ & Nsamples & $\mathrm{E}\left[\beta^{0}\right]$ & $\mathrm{E}\left[\beta^{0}\right]-\sigma_{\beta^{0}}$ & $\mathrm{E}\left[\beta^{0}\right]+\sigma_{\beta^{0}}$ & $\mathrm{E}\left[\beta^{0}\right]_{w}$ \\
\hline & {$\left[{ }^{\circ}\right]$} & & {$[\mathrm{dB}]$} & {$[\mathrm{dB}]$} & {$[\mathrm{dB}]$} & {$[\mathrm{dB}]$} & & {$[\mathrm{dB}]$} & {$[\mathrm{dB}]$} & {$[\mathrm{dB}]$} & {$[\mathrm{dB}]$} \\
\hline 1 & 25 & 0 & & & & & 0 & & & & \\
\hline 2 & 27 & 0 & & & & & 18265964 & -3.43 & -7.66 & -1.33 & -2.89 \\
\hline 3 & 29 & 79799161 & -4.26 & -6.70 & -2.70 & -3.86 & $1.6856082 \mathrm{e}+08$ & -4.46 & -6.86 & -2.92 & -3.50 \\
\hline 4 & 31 & $3.3226462 \mathrm{e}+08$ & -4.30 & -6.83 & -2.71 & -3.74 & $5.4750309 \mathrm{e}+08$ & -4.50 & -6.96 & -2.94 & -3.86 \\
\hline 5 & 33 & $4.8710806 \mathrm{e}+08$ & -4.48 & -7.04 & -2.89 & -3.96 & $8.3407165 e+08$ & -5.08 & -7.31 & -3.62 & -4.16 \\
\hline 6 & 35 & $6.0331673 \mathrm{e}+08$ & -5.00 & -7.56 & -3.41 & -4.35 & $1.2022899 \mathrm{e}+09$ & -5.51 & -7.73 & -4.05 & -4.59 \\
\hline 7 & 37 & $7.0295508 \mathrm{e}+08$ & -5.30 & -7.91 & -3.68 & -4.55 & $1.2942663 \mathrm{e}+09$ & -5.67 & -7.86 & -4.22 & -4.81 \\
\hline 8 & 39 & $8.4238833 \mathrm{e}+08$ & -5.53 & -8.15 & -3.91 & -4.83 & $1.1968624 \mathrm{e}+09$ & -6.01 & -8.25 & -4.55 & -5.11 \\
\hline 9 & 41 & $1.0139257 \mathrm{e}+09$ & -6.11 & -8.65 & -4.52 & -5.34 & $1.0404099 \mathrm{e}+09$ & -6.64 & -8.89 & -5.16 & -5.65 \\
\hline 10 & 43 & $1.5150585 \mathrm{e}+09$ & -6.56 & -9.02 & -5.00 & -5.66 & $5.9266559 \mathrm{e}+08$ & -7.06 & -9.38 & -5.56 & -5.95 \\
\hline 11 & 45 & $1.4532221 \mathrm{e}+09$ & -7.06 & -9.48 & -5.52 & -6.23 & $5.0377444 \mathrm{e}+08$ & -7.41 & -9.86 & -5.85 & -6.36 \\
\hline 12 & 47 & $1.1303886 \mathrm{e}+09$ & -7.35 & -9.65 & -5.85 & -6.75 & $6.0920658 \mathrm{e}+08$ & -7.29 & -9.73 & -5.73 & -6.37 \\
\hline \multirow[t]{5}{*}{13} & 49 & $1.9773776 \mathrm{e}+08$ & -7.42 & -9.37 & -6.08 & -7.01 & $1.6161167 \mathrm{e}+08$ & -7.26 & -9.77 & -5.68 & -6.38 \\
\hline & & & & \multicolumn{8}{|c|}{ Fitting Coefficients } \\
\hline & & & & \multicolumn{4}{|c|}{ Winter } & \multicolumn{4}{|c|}{ Summer } \\
\hline & & & & $c_{0}$ & $c_{1}$ & $c_{2}$ & $c_{3}$ & $c_{0}$ & $c_{1}$ & $c_{2}$ & $c_{3}$ \\
\hline & & & & -27.250166 & 109.27516 & -159.63121 & 69.521024 & -0.87439849 & 8.2914595 & -38.780689 & 25.032959 \\
\hline
\end{tabular}

Table A.5.: Class 5 - Closed to open ( $>15 \%)$ broadleaved evergreen or semi-deciduous forest $(>5 \mathrm{~m})$. Summary of backscatter statistics and fitting coefficients.

\begin{tabular}{|c|c|c|c|c|c|c|c|c|c|c|c|}
\hline \multirow{3}{*}{\multicolumn{2}{|c|}{$\begin{array}{c}\text { Class } 6 \\
\text { Continent } \\
\end{array}$}} & \multicolumn{10}{|c|}{ Closed $(>40 \%)$ broadleaved deciduous forest $(>5 \mathrm{~m})$} \\
\hline & & \multicolumn{10}{|c|}{ North America } \\
\hline & Season & \multicolumn{5}{|c|}{ Winter } & \multicolumn{5}{|c|}{ Summer } \\
\hline \multirow[t]{2}{*}{ Interval } & $\mathrm{E}[\theta]$ & Nsamples & $\mathrm{E}\left[\beta^{0}\right]$ & $\mathrm{E}\left[\beta^{0}\right]-\sigma_{\beta^{0}}$ & $\mathrm{E}\left[\beta^{0}\right]+\sigma_{\beta^{0}}$ & $\mathrm{E}\left[\beta^{0}\right]_{w}$ & Nsamples & $\mathrm{E}\left[\beta^{0}\right]$ & $\mathrm{E}\left[\beta^{0}\right]-\sigma_{\beta^{0}}$ & $\mathrm{E}\left[\beta^{0}\right]+\sigma_{\beta^{0}}$ & $\mathrm{E}\left[\beta^{0}\right]_{w}$ \\
\hline & {$\left[{ }^{\circ}\right]$} & & {$[\mathrm{dB}]$} & {$[\mathrm{dB}]$} & {$[\mathrm{dB}]$} & {$[\mathrm{dB}]$} & & {$[\mathrm{dB}]$} & {$[\mathrm{dB}]$} & {$[\mathrm{dB}]$} & {$[\mathrm{dB}]$} \\
\hline 1 & 25 & 0 & & & & & 0 & & & & \\
\hline 2 & 27 & 0 & & & & & 35049 & -4.75 & -8.94 & -2.66 & -3.57 \\
\hline 3 & 29 & 2656424 & -5.28 & -8.83 & -3.35 & -5.30 & 218082 & -4.62 & -8.90 & -2.51 & -4.32 \\
\hline 4 & 31 & 21264857 & -6.10 & -9.48 & -4.23 & -5.94 & 4116493 & -4.91 & -8.73 & -2.91 & -4.77 \\
\hline 5 & 33 & $1.3600549 \mathrm{e}+08$ & -6.71 & -9.65 & -4.97 & -6.41 & 21421497 & -5.99 & -9.00 & -4.23 & -5.59 \\
\hline 6 & 35 & $2.0125225 \mathrm{e}+08$ & -7.19 & -10.08 & -5.47 & -6.85 & 65277849 & -6.93 & -9.39 & -5.37 & -6.49 \\
\hline 7 & 37 & $1.2301064 \mathrm{e}+08$ & -7.40 & -10.19 & -5.71 & -6.85 & $1.9320366 \mathrm{e}+08$ & -7.14 & -9.60 & -5.58 & -6.74 \\
\hline 8 & 39 & 83669896 & -7.62 & -10.69 & -5.85 & -6.86 & $4.7158575 e+08$ & -7.37 & -9.91 & -5.78 & -6.96 \\
\hline 9 & 41 & 76868706 & -8.17 & -11.23 & -6.40 & -7.58 & $6.1718152 \mathrm{e}+08$ & -7.88 & -10.47 & -6.27 & -7.41 \\
\hline 10 & 43 & $1.0211510 \mathrm{e}+08$ & -8.60 & -11.49 & -6.88 & -8.01 & $6.8404561 \mathrm{e}+08$ & -8.18 & -10.80 & -6.56 & -7.66 \\
\hline 11 & 45 & $1.7677701 \mathrm{e}+08$ & -9.04 & -11.83 & -7.35 & -8.56 & $5.3570274 \mathrm{e}+08$ & -8.55 & -11.11 & -6.96 & -8.18 \\
\hline 12 & 47 & $1.8006358 \mathrm{e}+08$ & -9.23 & -12.08 & -7.53 & -9.01 & $2.7722788 \mathrm{e}+08$ & -8.81 & -11.40 & -7.20 & -8.53 \\
\hline \multirow[t]{5}{*}{13} & 49 & 20046683 & -9.23 & -12.24 & -7.47 & -9.54 & 15968214 & -8.62 & -11.35 & -6.96 & -8.66 \\
\hline & & & & \multicolumn{8}{|c|}{ Fitting Coefficients } \\
\hline & & & & \multicolumn{4}{|c|}{ Winter } & \multicolumn{4}{|c|}{ Summer } \\
\hline & & & & $c_{0}$ & $c_{1}$ & $c_{2}$ & $c_{3}$ & $c_{0}$ & $c_{1}$ & $c_{2}$ & $c_{3}$ \\
\hline & & & & 35.283383 & -176.87532 & 256.70482 & -130.20561 & 14.967299 & -59.722389 & 50.458019 & -15.152014 \\
\hline
\end{tabular}

Table A.6.: Class 6 - Closed $(>40 \%)$ broadleaved deciduous forest $(>5 \mathrm{~m})$. Summary of backscatter statistics and fitting coefficients. 


\begin{tabular}{|c|c|c|c|c|c|c|c|c|c|c|c|}
\hline \multirow{2}{*}{\multicolumn{2}{|c|}{$\begin{array}{c}\text { Class } 7 \\
\text { Continent }\end{array}$}} & \multicolumn{10}{|c|}{ Open $(15-40 \%)$ broadleaved deciduous forest/woodland $(>5 \mathrm{~m})$} \\
\hline & & \multicolumn{10}{|c|}{$\begin{array}{ll}\text { Africa } \\
\end{array}$} \\
\hline \multicolumn{2}{|c|}{ Season } & \multicolumn{5}{|c|}{ Winter } & \multicolumn{5}{|c|}{ Summer } \\
\hline \multirow[t]{2}{*}{ Interval } & $\mathrm{E}[\theta]$ & Nsamples & $\mathrm{E}\left[\beta^{0}\right]$ & $\mathrm{E}\left[\beta^{0}\right]-\sigma_{\beta^{0}}$ & $\mathrm{E}\left[\beta^{0}\right]+\sigma_{\beta^{0}}$ & $\mathrm{E}\left[\beta^{0}\right]_{w}$ & Nsamples & $\mathrm{E}\left[\beta^{0}\right]$ & $\mathrm{E}\left[\beta^{0}\right]-\sigma_{\beta^{0}}$ & $\mathrm{E}\left[\beta^{0}\right]+\sigma_{\beta^{0}}$ & $\mathrm{E}\left[\beta^{0}\right]_{w}$ \\
\hline & {$\left[{ }^{\circ}\right]$} & & {$[\mathrm{dB}]$} & {$[\mathrm{dB}]$} & {$[\mathrm{dB}]$} & {$[\mathrm{dB}]$} & & {$[\mathrm{dB}]$} & {$[\mathrm{dB}]$} & {$[\mathrm{dB}]$} & {$[\mathrm{dB}]$} \\
\hline 1 & 25 & 0 & & & & & 19389 & -5.04 & -9.11 & -2.97 & -5.30 \\
\hline 2 & 27 & 48 & -3.50 & -5.21 & -2.27 & -3.50 & 2877878 & -4.09 & -6.98 & -2.37 & -3.90 \\
\hline 3 & 29 & $1.2452387 \mathrm{e}+08$ & -5.47 & -7.46 & -4.11 & -5.14 & 37216398 & -6.29 & -8.40 & -4.87 & -5.90 \\
\hline 4 & 31 & $2.3444251 \mathrm{e}+08$ & -5.79 & -7.82 & -4.42 & -5.53 & $1.6551423 \mathrm{e}+08$ & -6.51 & -8.80 & -5.02 & -6.54 \\
\hline 5 & 33 & $1.9262088 \mathrm{e}+08$ & -6.12 & -8.31 & -4.67 & -5.80 & $2.4742539 \mathrm{e}+08$ & -6.96 & -9.19 & -5.49 & -6.87 \\
\hline 6 & 35 & $1.2013714 \mathrm{e}+08$ & -6.56 & -8.93 & -5.04 & -6.23 & $3.6170292 \mathrm{e}+08$ & -7.13 & -9.25 & -5.71 & -6.81 \\
\hline 7 & 37 & 65173961 & -6.78 & -9.29 & -5.21 & -6.25 & $3.5432663 e+08$ & -7.05 & -9.10 & -5.66 & -6.40 \\
\hline 8 & 39 & 44672175 & -7.00 & -9.72 & -5.35 & -6.21 & $4.0246817 \mathrm{e}+08$ & -7.28 & -9.51 & -5.81 & -6.44 \\
\hline 9 & 41 & 89249731 & -7.16 & -9.53 & -5.64 & -6.78 & $5.8486903 \mathrm{e}+08$ & -7.35 & -9.30 & -6.00 & -6.88 \\
\hline 10 & 43 & $1.5886912 \mathrm{e}+08$ & -7.65 & -9.77 & -6.23 & -7.27 & $4.7530699 \mathrm{e}+08$ & -7.68 & -9.62 & -6.34 & -7.07 \\
\hline 11 & 45 & $3.5407992 \mathrm{e}+08$ & -8.10 & -10.04 & -6.77 & -7.80 & $2.1510955 \mathrm{e}+08$ & -8.23 & -10.19 & -6.88 & -7.58 \\
\hline 12 & 47 & $4.9369244 \mathrm{e}+08$ & -8.45 & -10.31 & -7.16 & -8.18 & 85732994 & -8.62 & -10.62 & -7.25 & -8.24 \\
\hline \multirow[t]{5}{*}{13} & 49 & 98290947 & -8.67 & -10.44 & -7.41 & -8.40 & 34441250 & -8.82 & -10.73 & -7.49 & -8.32 \\
\hline & & & & \multicolumn{8}{|c|}{ Fitting Coefficients } \\
\hline & & & & \multicolumn{4}{|c|}{ Winter } & \multicolumn{4}{|c|}{ Summer } \\
\hline & & & & $c_{0}$ & $c_{1}$ & $c_{2}$ & $c_{3}$ & $c_{0}$ & $c_{1}$ & $c_{2}$ & $c_{3}$ \\
\hline & & & & 68.984372 & -325.79480 & 474.73131 & -233.96337 & 0.0062252356 & -14.524795 & 9.7675674 & -4.7709718 \\
\hline
\end{tabular}

Table A.7.: Class 7 - Open (15-40\%) broadleaved deciduous forest/woodland ( $>5 \mathrm{~m})$. Summary of backscatter statistics and fitting coefficients.

\begin{tabular}{|c|c|c|c|c|c|c|c|c|c|c|c|}
\hline \multirow{3}{*}{\multicolumn{2}{|c|}{$\begin{array}{c}\text { Class } 8 \\
\text { Continent } \\
\text { Season }\end{array}$}} & \multicolumn{10}{|c|}{ Closed $(>40 \%)$ needleleaved evergreen forest $(>5 \mathrm{~m})$} \\
\hline & & \multicolumn{10}{|c|}{$\begin{array}{ll}\text { North America } \\
\end{array}$} \\
\hline & & \multicolumn{5}{|c|}{ Winter } & \multicolumn{5}{|c|}{ Summer } \\
\hline \multirow[t]{2}{*}{ Interval } & $\mathrm{E}[\theta]$ & Nsamples & $\mathrm{E}\left[\beta^{0}\right]$ & $\mathrm{E}\left[\beta^{0}\right]-\sigma_{\beta^{0}}$ & $\mathrm{E}\left[\beta^{0}\right]+\sigma_{\beta^{0}}$ & $\mathrm{E}\left[\beta^{0}\right]_{w}$ & Nsamples & $\mathrm{E}\left[\beta^{0}\right]$ & $\mathrm{E}\left[\beta^{0}\right]-\sigma_{\beta^{0}}$ & $\mathrm{E}\left[\beta^{0}\right]+\sigma_{\beta^{0}}$ & $\mathrm{E}\left[\beta^{0}\right]_{w}$ \\
\hline & {$\left[{ }^{\circ}\right]$} & & {$[\mathrm{dB}]$} & {$[\mathrm{dB}]$} & {$[\mathrm{dB}]$} & {$[\mathrm{dB}]$} & & {$[\mathrm{dB}]$} & {$[\mathrm{dB}]$} & {$[\mathrm{dB}]$} & {$[\mathrm{dB}]$} \\
\hline 1 & 25 & 0 & & & & & 0 & & & & \\
\hline 2 & 27 & 44489 & -5.02 & $\mathrm{~N}$ & -1.92 & -4.98 & 355859 & -3.02 & -9.67 & -0.50 & -3.73 \\
\hline 3 & 29 & 10330643 & -5.70 & -10.29 & -3.52 & -5.73 & 1476392 & -4.63 & -9.42 & -2.41 & -4.38 \\
\hline 4 & 31 & 36514880 & -6.22 & -10.14 & -4.20 & -6.19 & 9514410 & -5.14 & -9.19 & -3.09 & -5.05 \\
\hline 5 & 33 & $1.3265276 \mathrm{e}+08$ & -6.74 & -10.34 & -4.79 & -6.42 & 19055510 & -6.63 & -9.80 & -4.82 & -5.87 \\
\hline 6 & 35 & $2.2325119 \mathrm{e}+08$ & -7.08 & -10.40 & -5.22 & -6.71 & 97252766 & -7.97 & -11.10 & -6.17 & -6.90 \\
\hline 7 & 37 & $2.0581615 \mathrm{e}+08$ & -7.20 & -10.29 & -5.41 & -6.81 & $2.1940824 \mathrm{e}+08$ & -8.11 & -11.31 & -6.28 & -7.11 \\
\hline 8 & 39 & $1.9331319 \mathrm{e}+08$ & -7.39 & -10.50 & -5.59 & -6.92 & $3.9069100 \mathrm{e}+08$ & -8.24 & -11.46 & -6.42 & -7.29 \\
\hline 9 & 41 & $2.1397059 \mathrm{e}+08$ & -8.00 & -11.06 & -6.23 & -7.56 & $5.1020847 \mathrm{e}+08$ & -8.65 & -11.86 & -6.82 & -7.80 \\
\hline 10 & 43 & $2.2104670 \mathrm{e}+08$ & -8.33 & -11.26 & -6.60 & -7.92 & $5.0557127 \mathrm{e}+08$ & -8.94 & -12.05 & -7.14 & -8.13 \\
\hline 11 & 45 & $2.7798078 \mathrm{e}+08$ & -8.71 & -11.65 & -6.97 & -8.36 & $4.2560110 \mathrm{e}+08$ & -9.11 & -12.14 & -7.34 & -8.64 \\
\hline 12 & 47 & $1.7620833 \mathrm{e}+08$ & -9.14 & -12.24 & -7.35 & -8.97 & $1.9861157 \mathrm{e}+08$ & -9.21 & -12.23 & -7.44 & -8.91 \\
\hline \multirow[t]{5}{*}{13} & 49 & 14627644 & -9.46 & -12.64 & -7.64 & -9.51 & 7290401 & -8.16 & -13.44 & -5.85 & -8.11 \\
\hline & & & & \multicolumn{8}{|c|}{ Fitting Coefficients } \\
\hline & & & & \multicolumn{4}{|c|}{ Winter } & \multicolumn{4}{|c|}{ Summer } \\
\hline & & & & $c_{0}$ & $c_{1}$ & $c_{2}$ & $c_{3}$ & $c_{0}$ & $c_{1}$ & $c_{2}$ & $c_{3}$ \\
\hline & & & & 36.504362 & -188.28656 & 280.79258 & -144.67405 & -3.9707555 & 38.557409 & -117.07262 & 77.272233 \\
\hline
\end{tabular}

Table A.8.: Class 8 - Closed ( $>40 \%$ ) needleleaved evergreen forest ( $>5 \mathrm{~m})$. Summary of backscatter statistics and fitting coefficients. 


\begin{tabular}{|c|c|c|c|c|c|c|c|c|c|c|c|}
\hline \multirow{2}{*}{\multicolumn{2}{|c|}{$\begin{array}{c}\text { Class } 9 \\
\text { Continent }\end{array}$}} & \multicolumn{10}{|c|}{ Open $(15-40 \%)$ needleleaved deciduous or evergreen forest $(>5 \mathrm{~m})$} \\
\hline & & \multicolumn{10}{|c|}{ Asia } \\
\hline \multicolumn{2}{|c|}{ Season } & \multicolumn{5}{|c|}{ Winter } & \multicolumn{5}{|c|}{ Summer } \\
\hline \multirow[t]{2}{*}{ Interval } & $\mathrm{E}[\theta]$ & Nsamples & $\mathrm{E}\left[\beta^{0}\right]$ & $\mathrm{E}\left[\beta^{0}\right]-\sigma_{\beta^{0}}$ & $\mathrm{E}\left[\beta^{0}\right]+\sigma_{\beta^{0}}$ & $\mathrm{E}\left[\beta^{0}\right]_{w}$ & Nsamples & $\mathrm{E}\left[\beta^{0}\right]$ & $\mathrm{E}\left[\beta^{0}\right]-\sigma_{\beta^{0}}$ & $\mathrm{E}\left[\beta^{0}\right]+\sigma_{\beta^{0}}$ & $\mathrm{E}\left[\beta^{0}\right]_{w}$ \\
\hline & {$\left[{ }^{\circ}\right]$} & & {$[\mathrm{dB}]$} & {$[\mathrm{dB}]$} & {$[\mathrm{dB}]$} & {$[\mathrm{dB}]$} & & {$[\mathrm{dB}]$} & {$[\mathrm{dB}]$} & {$[\mathrm{dB}]$} & {$[\mathrm{dB}]$} \\
\hline 1 & 25 & 0 & & & & & 0 & & & & \\
\hline 2 & 27 & 0 & & & & & 0 & & & & \\
\hline 3 & 29 & 0 & & & & & 7 & -1.74 & $\mathrm{~N}$ & 1.8 & -1.74 \\
\hline 4 & 31 & 657 & -4.37 & -7.94 & -2.44 & -4.27 & 228366 & -3.21 & -7.52 & -1.09 & -3.21 \\
\hline 5 & 33 & 26506660 & -6.24 & -9.84 & -4.30 & -5.59 & 53339773 & -6.16 & -8.25 & -4.75 & -6.03 \\
\hline 6 & 35 & $3.8594683 \mathrm{e}+08$ & -6.82 & -9.64 & -5.12 & -6.26 & $9.7041887 \mathrm{e}+08$ & -7.34 & -9.18 & -6.05 & -6.90 \\
\hline 7 & 37 & $7.6435471 \mathrm{e}+08$ & -7.06 & -9.59 & -5.48 & -6.53 & $2.5006465 \mathrm{e}+09$ & -7.63 & -9.61 & -6.28 & -7.29 \\
\hline 8 & 39 & $9.3527104 \mathrm{e}+08$ & -7.46 & -10.02 & -5.86 & -6.81 & $2.4247238 \mathrm{e}+09$ & -7.95 & -10.01 & -6.56 & -7.53 \\
\hline 9 & 41 & $1.1111456 \mathrm{e}+09$ & -7.92 & -10.39 & -6.36 & -7.31 & $2.0777946 \mathrm{e}+09$ & -8.37 & -10.52 & -6.93 & -7.92 \\
\hline 10 & 43 & $1.0521840 \mathrm{e}+09$ & -8.23 & -10.65 & -6.69 & -7.68 & $1.5002287 \mathrm{e}+09$ & -8.57 & -10.73 & -7.14 & -8.20 \\
\hline 11 & 45 & $6.5717924 \mathrm{e}+08$ & -8.56 & -11.05 & -6.99 & -7.96 & $8.4316126 \mathrm{e}+08$ & -8.58 & -10.69 & -7.16 & -8.41 \\
\hline 12 & 47 & 99308170 & -8.98 & -11.70 & -7.33 & -8.70 & 77807578 & -8.67 & -11.14 & -7.10 & -8.43 \\
\hline \multirow[t]{5}{*}{13} & 49 & 2452671 & -8.73 & -11.46 & -7.07 & -8.85 & 39080 & -8.46 & $\mathrm{~N}$ & -5.32 & -7.90 \\
\hline & & & & \multicolumn{8}{|c|}{ Fitting Coefficients } \\
\hline & & & & \multicolumn{4}{|c|}{ Winter } & \multicolumn{4}{|c|}{ Summer } \\
\hline & & & & $c_{0}$ & $c_{1}$ & $c_{2}$ & $c_{3}$ & $c_{0}$ & $c_{1}$ & $c_{2}$ & $c_{3}$ \\
\hline & & & & 100.66327 & -430.91397 & 582.43011 & -267.20369 & 79.861417 & -290.49900 & 307.21942 & -102.38058 \\
\hline
\end{tabular}

Table A.9.: Class 9 - Open (15-40\%) needleleaved deciduous or evergreen forest $(>5 \mathrm{~m})$. Summary of backscatter statistics and fitting coefficients.

\begin{tabular}{|c|c|c|c|c|c|c|c|c|c|c|c|}
\hline \multirow{2}{*}{\multicolumn{2}{|c|}{$\begin{array}{c}\text { Class 10 } \\
\text { Continent }\end{array}$}} & \multicolumn{10}{|c|}{ Closed to open ( $>15 \%)$ mixed broadleaved and needleleaved forest $(>5 \mathrm{~m})$} \\
\hline & & \multicolumn{10}{|c|}{ North America } \\
\hline \multicolumn{2}{|c|}{ Season } & \multicolumn{5}{|c|}{ Winter } & \multicolumn{5}{|c|}{ Summer } \\
\hline Interval & $\mathrm{E}[\theta]$ & Nsamples & $\mathrm{E}\left[\beta^{0}\right]$ & $\mathrm{E}\left[\beta^{0}\right]-\sigma_{\beta^{0}}$ & $\mathrm{E}\left[\beta^{0}\right]+\sigma_{\beta^{0}}$ & $\mathrm{E}\left[\beta^{0}\right]_{w}$ & Nsamples & $\mathrm{E}\left[\beta^{0}\right]$ & $\mathrm{E}\left[\beta^{0}\right]-\sigma_{\beta^{0}}$ & $\mathrm{E}\left[\beta^{0}\right]+\sigma_{\beta^{0}}$ & $\mathrm{E}\left[\beta^{0}\right]_{w}$ \\
\hline & {$\left[{ }^{\circ}\right]$} & & {$[\mathrm{dB}]$} & {$[\mathrm{dB}]$} & {$[\mathrm{dB}]$} & {$[\mathrm{dB}]$} & & {$[\mathrm{dB}]$} & {$[\mathrm{dB}]$} & {$[\mathrm{dB}]$} & {$[\mathrm{dB}]$} \\
\hline 1 & 25 & 0 & & & & & 0 & & & & \\
\hline 2 & 27 & 1893 & -4.95 & -17.47 & -2.06 & -5.06 & 34210 & -3.40 & -10.32 & -0.86 & -3.91 \\
\hline 3 & 29 & 779825 & -5.54 & -9.81 & -3.43 & -5.25 & 152098 & -4.41 & -8.78 & -2.27 & -4.45 \\
\hline 4 & 31 & 4877874 & -6.11 & -9.71 & -4.17 & -5.88 & 1360747 & -4.88 & -8.88 & -2.84 & -4.96 \\
\hline 5 & 33 & 36438549 & -6.55 & -9.76 & -4.72 & -6.18 & 12194018 & -6.20 & -8.71 & -4.62 & -5.86 \\
\hline 6 & 35 & $1.0369405 \mathrm{e}+08$ & -6.80 & -9.89 & -5.01 & -6.37 & $1.4677108 \mathrm{e}+08$ & -7.12 & -8.94 & -5.83 & -6.73 \\
\hline 7 & 37 & $1.2992932 \mathrm{e}+08$ & -7.05 & -9.95 & -5.33 & -6.51 & $3.0263535 \mathrm{e}+08$ & -7.33 & -9.32 & -5.96 & -6.94 \\
\hline 8 & 39 & $1.5564493 \mathrm{e}+08$ & -7.34 & -10.19 & -5.63 & -6.70 & $3.8086764 \mathrm{e}+08$ & -7.55 & -9.63 & -6.15 & -7.18 \\
\hline 9 & 41 & $1.9397116 \mathrm{e}+08$ & -7.83 & -10.56 & -6.17 & -7.29 & $4.0205487 \mathrm{e}+08$ & -8.04 & -10.27 & -6.57 & -7.69 \\
\hline 10 & 43 & $2.0652069 \mathrm{e}+08$ & -8.19 & -10.85 & -6.56 & -7.68 & $3.3997099 \mathrm{e}+08$ & -8.43 & -10.73 & -6.93 & -8.02 \\
\hline 11 & 45 & $1.9593416 \mathrm{e}+08$ & -8.57 & -11.22 & -6.94 & -8.03 & $2.4775627 \mathrm{e}+08$ & -8.70 & -11.04 & -7.19 & -8.30 \\
\hline 12 & 47 & $1.0139103 \mathrm{e}+08$ & -9.06 & -11.77 & -7.40 & -8.34 & 62799455 & -8.90 & -11.36 & -7.34 & -8.70 \\
\hline \multirow[t]{5}{*}{13} & 49 & 4822569 & -9.48 & -12.37 & -7.76 & -9.24 & 1993095 & -8.05 & -13.13 & -5.77 & -7.76 \\
\hline & & & & \multicolumn{8}{|c|}{ "Fitting Coefficients } \\
\hline & & & & \multicolumn{4}{|c|}{ Winter } & \multicolumn{4}{|c|}{ Summer } \\
\hline & & & & $c_{0}$ & $c_{1}$ & $c_{2}$ & $c_{3}$ & $c_{0}$ & $c_{1}$ & $c_{2}$ & $c_{3}$ \\
\hline & & & & 16.023288 & -93.770826 & 139.60497 & -75.321195 & -16.981116 & 97.371460 & -204.49449 & 120.46786 \\
\hline
\end{tabular}

Table A.10.: Class 10 - Closed to open (>15\%) mixed broadleaved and needleleaved forest $(>5 \mathrm{~m})$. Summary of backscatter statistics and fitting coefficients. 


\begin{tabular}{|c|c|c|c|c|c|c|c|c|c|c|c|}
\hline \multirow{3}{*}{\multicolumn{2}{|c|}{$\begin{array}{c}\text { Class 11 } \\
\text { Continent } \\
\text { Season }\end{array}$}} & \multicolumn{10}{|c|}{ Mosaic forest or shrubland $(50-70 \%)$ / grassland $(20-50 \%)$} \\
\hline & & \multicolumn{10}{|c|}{ North America } \\
\hline & & \multicolumn{5}{|c|}{ Winter } & \multicolumn{5}{|c|}{ Summer } \\
\hline \multirow[t]{2}{*}{ Interval } & $\mathrm{E}[\theta]$ & Nsamples & $\mathrm{E}\left[\beta^{0}\right]$ & $\mathrm{E}\left[\beta^{0}\right]-\sigma_{\beta^{0}}$ & $\mathrm{E}\left[\beta^{0}\right]+\sigma_{\beta^{0}}$ & $\mathrm{E}\left[\beta^{0}\right]_{w}$ & Nsamples & $\mathrm{E}\left[\beta^{0}\right]$ & $\mathrm{E}\left[\beta^{0}\right]-\sigma_{\beta^{0}}$ & $\mathrm{E}\left[\beta^{0}\right]+\sigma_{\beta^{0}}$ & $\mathrm{E}\left[\beta^{0}\right]_{w}$ \\
\hline & {$\left[{ }^{\circ}\right]$} & & {$[\mathrm{dB}]$} & {$[\mathrm{dB}]$} & {$[\mathrm{dB}]$} & {$[\mathrm{dB}]$} & & {$[\mathrm{dB}]$} & {$[\mathrm{dB}]$} & {$[\mathrm{dB}]$} & {$[\mathrm{dB}]$} \\
\hline 1 & 25 & 0 & & & & & 0 & & & & \\
\hline 2 & 27 & 133174 & -4.25 & -8.51 & -2.14 & -4.28 & 53372 & -2.74 & -7.06 & -0.62 & -2.42 \\
\hline 3 & 29 & 9000030 & -5.06 & -8.65 & -3.12 & -5.22 & 708360 & -4.03 & -8.45 & -1.89 & -3.91 \\
\hline 4 & 31 & 27819447 & -5.90 & -9.61 & -3.93 & -6.02 & 1627071 & -4.90 & -8.92 & -2.84 & -4.66 \\
\hline 5 & 33 & 59750320 & -6.67 & -10.15 & -4.76 & -6.75 & 6919172 & -6.54 & -9.57 & -4.77 & -6.37 \\
\hline 6 & 35 & $1.0434636 \mathrm{e}+08$ & -7.16 & -10.60 & -5.26 & -7.58 & $1.2965719 \mathrm{e}+08$ & -7.50 & -9.79 & -6.00 & -7.19 \\
\hline 7 & 37 & $1.5170356 \mathrm{e}+08$ & -7.51 & -10.71 & -5.69 & -7.74 & $3.5385225 \mathrm{e}+08$ & -7.75 & -10.23 & -6.19 & -7.46 \\
\hline 8 & 39 & $1.8898798 \mathrm{e}+08$ & -7.85 & -11.06 & -6.02 & -7.59 & $4.0764776 \mathrm{e}+08$ & -7.99 & -10.53 & -6.40 & -7.82 \\
\hline 9 & 41 & $2.5438174 \mathrm{e}+08$ & -8.49 & -11.57 & -6.71 & -7.99 & $4.1321849 \mathrm{e}+08$ & -8.49 & -11.17 & -6.84 & -8.40 \\
\hline 10 & 43 & $2.7782937 \mathrm{e}+08$ & -8.63 & -11.75 & -6.83 & -8.25 & $3.7198031 \mathrm{e}+08$ & -8.89 & -11.68 & -7.20 & -8.91 \\
\hline 11 & 45 & $1.5090100 \mathrm{e}+08$ & -8.83 & -11.87 & -7.05 & -8.45 & $2.5539971 \mathrm{e}+08$ & -9.16 & -12.02 & -7.45 & -9.28 \\
\hline 12 & 47 & 74575056 & -9.24 & -12.42 & -7.43 & -9.37 & 86651067 & -9.32 & -12.29 & -7.57 & -10.43 \\
\hline \multirow[t]{5}{*}{13} & 49 & 6510353 & -9.40 & -12.67 & -7.56 & -9.49 & 5804058 & -8.54 & -11.72 & -6.72 & -8.58 \\
\hline & & & & \multicolumn{8}{|c|}{ Fitting Coefficients } \\
\hline & & & & \multicolumn{4}{|c|}{ Winter } & \multicolumn{4}{|c|}{ Summer } \\
\hline & & & & $c_{0}$ & $c_{1}$ & $c_{2}$ & $c_{3}$ & $c_{0}$ & $c_{1}$ & $c_{2}$ & $c_{3}$ \\
\hline & & & & 57.028071 & -263.42576 & 364.26024 & -172.25963 & 23.219589 & -65.066300 & 10.746210 & 24.789422 \\
\hline
\end{tabular}

Table A.11.: Class 11 - Mosaic forest or shrubland (50-70\%) / grassland (20-50\%). Summary of backscatter statistics and fitting coefficients.

\begin{tabular}{|c|c|c|c|c|c|c|c|c|c|c|c|}
\hline \multirow{3}{*}{\multicolumn{2}{|c|}{$\begin{array}{c}\text { Class } 12 \\
\text { Continent } \\
\text { Season }\end{array}$}} & \multicolumn{10}{|c|}{ Mosaic grassland $(50-70 \%)$ / forest or shrubland $(20-50 \%)$} \\
\hline & & \multicolumn{10}{|c|}{$\begin{array}{ll}\text { Europe } \\
\end{array}$} \\
\hline & & \multicolumn{5}{|c|}{ Winter } & \multicolumn{5}{|c|}{ Summer } \\
\hline \multirow[t]{2}{*}{ Interval } & $\mathrm{E}[\theta]$ & Nsamples & $\mathrm{E}\left[\beta^{0}\right]$ & $\mathrm{E}\left[\beta^{0}\right]-\sigma_{\beta^{0}}$ & $\mathrm{E}\left[\beta^{0}\right]+\sigma_{\beta^{0}}$ & $\mathrm{E}\left[\beta^{0}\right]_{w}$ & Nsamples & $\mathrm{E}\left[\beta^{0}\right]$ & $\mathrm{E}\left[\beta^{0}\right]-\sigma_{\beta^{0}}$ & $\mathrm{E}\left[\beta^{0}\right]+\sigma_{\beta^{0}}$ & $\mathrm{E}\left[\beta^{0}\right]_{w}$ \\
\hline & {$\left[{ }^{\circ}\right]$} & & {$[\mathrm{dB}]$} & {$[\mathrm{dB}]$} & {$[\mathrm{dB}]$} & {$[\mathrm{dB}]$} & & {$[\mathrm{dB}]$} & {$[\mathrm{dB}]$} & {$[\mathrm{dB}]$} & {$[\mathrm{dB}]$} \\
\hline 1 & 25 & 0 & & & & & 0 & & & & \\
\hline 2 & 27 & 0 & & & & & 0 & & & & \\
\hline 3 & 29 & 0 & & & & & 0 & & & & \\
\hline 4 & 31 & 78271 & -4.60 & -11.82 & -2.02 & -4.90 & 326149 & -5.77 & -8.90 & -3.97 & -5.78 \\
\hline 5 & 33 & 15941627 & -6.16 & -10.08 & -4.13 & -5.99 & 3171626 & -6.04 & -9.41 & -4.17 & -5.94 \\
\hline 6 & 35 & 54557360 & -6.64 & -10.67 & -4.58 & -6.46 & 23403273 & -7.21 & -10.07 & -5.50 & -6.89 \\
\hline 7 & 37 & $1.0484369 \mathrm{e}+08$ & -7.27 & -10.57 & -5.42 & -6.87 & 41290024 & -7.48 & -10.54 & -5.71 & -7.23 \\
\hline 8 & 39 & $2.0695320 \mathrm{e}+08$ & -7.86 & -10.94 & -6.08 & -7.25 & 68962994 & -7.69 & -10.90 & -5.87 & -7.34 \\
\hline 9 & 41 & $2.4558354 \mathrm{e}+08$ & -8.45 & -11.28 & -6.76 & -7.92 & 99190592 & -8.14 & -11.69 & -6.21 & -7.94 \\
\hline 10 & 43 & $2.6603379 \mathrm{e}+08$ & -8.62 & -11.44 & -6.93 & -8.14 & 96066505 & -8.44 & -12.14 & -6.47 & -8.30 \\
\hline 11 & 45 & 92457061 & -8.95 & -12.19 & -7.12 & -8.59 & 76473965 & -8.74 & -12.54 & -6.75 & -8.71 \\
\hline 12 & 47 & 31136372 & -9.41 & -13.14 & -7.43 & -9.29 & 24495292 & -9.05 & -12.83 & -7.06 & -8.99 \\
\hline \multirow[t]{5}{*}{13} & 49 & 217685 & -10.12 & -13.31 & -8.29 & -9.88 & 76110 & -11.53 & -18.01 & -9.03 & -10.81 \\
\hline & & & & \multicolumn{8}{|c|}{ Fitting Coefficients } \\
\hline & & & & \multicolumn{4}{|c|}{ Winter } & \multicolumn{4}{|c|}{ Summer } \\
\hline & & & & $c_{0}$ & $c_{1}$ & $c_{2}$ & $c_{3}$ & $c_{0}$ & $c_{1}$ & $c_{2}$ & $c_{3}$ \\
\hline & & & & 75.485186 & -331.06096 & 452.31839 & -212.80707 & 78.797560 & -369.13275 & 538.99156 & -268.49752 \\
\hline
\end{tabular}

Table A.12.: Class 12 - Mosaic grassland (50-70\%) / forest or shrubland (20-50\%). Summary of backscatter statistics and fitting coefficients. 


\begin{tabular}{|c|c|c|c|c|c|c|c|c|c|c|c|}
\hline \multirow{3}{*}{\multicolumn{2}{|c|}{$\begin{array}{c}\text { Class 13 } \\
\text { Continent } \\
\text { Season }\end{array}$}} & \multicolumn{10}{|c|}{ Closed to open ( $>15 \%)$ (broadleaved or needleleaved, evergreen or deciduous) shrubland $(<5 \mathrm{~m})$} \\
\hline & & \multicolumn{10}{|c|}{ North America } \\
\hline & & \multicolumn{5}{|c|}{ Winter } & \multicolumn{5}{|c|}{ Summer } \\
\hline \multirow[t]{2}{*}{ Interval } & $\mathrm{E}[\theta]$ & Nsamples & $\mathrm{E}\left[\beta^{0}\right]$ & $\mathrm{E}\left[\beta^{0}\right]-\sigma_{\beta^{0}}$ & $\mathrm{E}\left[\beta^{0}\right]+\sigma_{\beta^{0}}$ & $\mathrm{E}\left[\beta^{0}\right]_{w}$ & Nsamples & $\mathrm{E}\left[\beta^{0}\right]$ & $\mathrm{E}\left[\beta^{0}\right]-\sigma_{\beta^{0}}$ & $\mathrm{E}\left[\beta^{0}\right]+\sigma_{\beta^{0}}$ & $\mathrm{E}\left[\beta^{0}\right]_{w}$ \\
\hline & {$\left[{ }^{\circ}\right]$} & & {$[\mathrm{dB}]$} & [dB] & {$[\mathrm{dB}]$} & {$[\mathrm{dB}]$} & & {$[\mathrm{dB}]$} & [dB] & [dB] & {$[\mathrm{dB}]$} \\
\hline 1 & 25 & 0 & & & & & 0 & & & & \\
\hline 2 & 27 & 0 & & & & & 21903 & -2.25 & -7.52 & 0.0 & -2.05 \\
\hline 3 & 29 & 6980548 & -5.83 & -10.05 & -3.73 & -5.91 & 1242272 & -4.23 & -7.57 & -2.37 & -4.36 \\
\hline 4 & 31 & 67413505 & -6.82 & -11.15 & -4.70 & -7.46 & 6351149 & -6.00 & -9.75 & -4.02 & -6.25 \\
\hline 5 & 33 & $2.1771577 \mathrm{e}+08$ & -7.56 & -11.57 & -5.51 & -8.28 & 31006976 & -6.89 & -10.67 & -4.90 & -6.93 \\
\hline 6 & 35 & $2.3347196 \mathrm{e}+08$ & -7.82 & -11.27 & -5.92 & -9.17 & 54667948 & -7.88 & -10.09 & -6.42 & -7.44 \\
\hline 7 & 37 & $2.1462035 e+08$ & -7.90 & -11.10 & -6.08 & -9.30 & $1.4301631 \mathrm{e}+08$ & -8.23 & -10.71 & -6.66 & -8.39 \\
\hline 8 & 39 & $1.6443203 \mathrm{e}+08$ & -7.94 & -10.94 & -6.17 & -8.91 & $3.5111798 \mathrm{e}+08$ & -8.38 & -11.12 & -6.71 & -9.00 \\
\hline 9 & 41 & $1.8096816 \mathrm{e}+08$ & -8.39 & -11.24 & -6.68 & -9.06 & $5.8826927 \mathrm{e}+08$ & -8.88 & -11.80 & -7.14 & -9.46 \\
\hline 10 & 43 & $1.7854825 \mathrm{e}+08$ & -8.69 & -11.55 & -6.98 & -9.27 & $6.3746408 \mathrm{e}+08$ & -9.16 & -12.21 & -7.39 & -9.97 \\
\hline 11 & 45 & $1.4134860 \mathrm{e}+08$ & -9.15 & -12.02 & -7.44 & -10.18 & $6.1875406 \mathrm{e}+08$ & -9.58 & -12.80 & -7.75 & -10.73 \\
\hline 12 & 47 & $1.5128341 \mathrm{e}+08$ & -9.66 & -13.04 & -7.79 & -11.02 & $3.9493287 \mathrm{e}+08$ & -9.88 & -13.44 & -7.95 & -11.18 \\
\hline \multirow[t]{5}{*}{13} & 49 & 20125058 & -10.02 & -13.82 & -8.03 & -10.74 & 33477455 & -9.04 & -12.73 & -7.07 & -9.56 \\
\hline & & & & \multicolumn{8}{|c|}{ Fitting Coefficients } \\
\hline & & & & \multicolumn{4}{|c|}{ Winter } & \multicolumn{4}{|c|}{ Summer } \\
\hline & & & & $c_{0}$ & $c_{1}$ & $c_{2}$ & $c_{3}$ & $c_{0}$ & $c_{1}$ & $c_{2}$ & $c_{3}$ \\
\hline & & & & 128.58744 & -585.35357 & 831.08784 & -394.86270 & 82.283958 & -331.79401 & 400.44288 & -162.52566 \\
\hline
\end{tabular}

Table A.13.: Class 13 - Closed to open ( $>15 \%)$ (broadleaved or needleleaved, evergreen or deciduous) shrubland $(<5 \mathrm{~m})$. Summary of backscatter statistics and fitting coefficients.

\begin{tabular}{|c|c|c|c|c|c|c|c|c|c|c|c|}
\hline \multirow{2}{*}{\multicolumn{2}{|c|}{$\begin{array}{c}\text { Class } 14 \\
\text { Continent }\end{array}$}} & \multicolumn{10}{|c|}{ Closed to open ( $>15 \%$ ) herbaceous vegetation (grassland, savannas or lichens/mosses) } \\
\hline & & \multicolumn{10}{|c|}{ Asia } \\
\hline \multicolumn{2}{|c|}{ Season } & \multicolumn{5}{|c|}{ Winter } & \multicolumn{5}{|c|}{ Summer } \\
\hline Interval & $\mathrm{E}[\theta]$ & Nsamples & $\mathrm{E}\left[\beta^{0}\right]$ & $\mathrm{E}\left[\beta^{0}\right]-\sigma_{\beta^{0}}$ & $\mathrm{E}\left[\beta^{0}\right]+\sigma_{\beta^{0}}$ & $\mathrm{E}\left[\beta^{0}\right]_{w}$ & Nsamples & $\mathrm{E}\left[\beta^{0}\right]$ & $\mathrm{E}\left[\beta^{0}\right]-\sigma_{\beta^{0}}$ & $\mathrm{E}\left[\beta^{0}\right]+\sigma_{\beta^{0}}$ & $\mathrm{E}\left[\beta^{0}\right]_{w}$ \\
\hline & {$\left[{ }^{\circ}\right]$} & & {$[\mathrm{dB}]$} & {$[\mathrm{dB}]$} & {$[\mathrm{dB}]$} & {$[\mathrm{dB}]$} & & {$[\mathrm{dB}]$} & {$[\mathrm{dB}]$} & {$[\mathrm{dB}]$} & {$[\mathrm{dB}]$} \\
\hline 1 & 25 & 2318701 & -6.37 & -12.33 & -3.95 & -7.43 & 4043561 & -5.64 & -11.50 & -3.23 & -5.85 \\
\hline 2 & 27 & 264510 & -6.42 & -10.67 & -4.32 & -6.26 & 6006495 & -6.69 & -12.10 & -4.35 & -7.17 \\
\hline 3 & 29 & 6654970 & -5.75 & -9.85 & -3.68 & -6.01 & 7027071 & -6.89 & -11.36 & -4.73 & -7.16 \\
\hline 4 & 31 & 49958869 & -6.17 & -11.51 & -3.85 & -7.24 & 10718310 & -5.95 & -11.27 & -3.63 & -7.54 \\
\hline 5 & 33 & $1.6044573 \mathrm{e}+08$ & -6.72 & -13.35 & -4.20 & -7.50 & 27018623 & -6.31 & -11.45 & -4.02 & -6.25 \\
\hline 6 & 35 & $1.8220638 \mathrm{e}+08$ & -7.27 & -13.75 & -4.77 & -8.53 & 37977899 & -7.27 & -11.42 & -5.18 & -7.02 \\
\hline 7 & 37 & $1.4161204 \mathrm{e}+08$ & -7.84 & -13.94 & -5.40 & -8.18 & 77162863 & -7.78 & -11.75 & -5.75 & -7.75 \\
\hline 8 & 39 & $1.5112478 \mathrm{e}+08$ & -8.11 & -14.50 & -5.62 & -8.66 & $1.9795097 \mathrm{e}+08$ & -7.94 & -12.09 & -5.86 & -8.27 \\
\hline 9 & 41 & $1.5078439 \mathrm{e}+08$ & -8.67 & -15.29 & -6.16 & -9.35 & $2.9568720 \mathrm{e}+08$ & -8.34 & -12.87 & -6.17 & -8.89 \\
\hline 10 & 43 & $1.3078513 \mathrm{e}+08$ & -9.05 & -15.36 & -6.58 & -9.94 & $3.3641724 \mathrm{e}+08$ & -8.58 & -13.40 & -6.35 & -9.49 \\
\hline 11 & 45 & $1.3143530 \mathrm{e}+08$ & -9.82 & -15.96 & -7.38 & -10.48 & $2.4026642 \mathrm{e}+08$ & -8.92 & -13.80 & -6.68 & -9.89 \\
\hline 12 & 47 & $1.2766388 \mathrm{e}+08$ & -10.03 & -16.22 & -7.57 & -11.08 & $1.0802660 \mathrm{e}+08$ & -9.01 & -14.16 & -6.72 & -9.83 \\
\hline \multirow[t]{5}{*}{13} & 49 & 14752307 & -9.77 & -15.89 & -7.33 & -10.17 & 15155609 & -8.76 & -15.72 & -6.21 & -9.78 \\
\hline & & & & \multicolumn{8}{|c|}{ Fitting Coefficients } \\
\hline & & & & \multicolumn{4}{|c|}{ Winter } & \multicolumn{4}{|c|}{ Summer } \\
\hline & & & & $c_{0}$ & $c_{1}$ & $c_{2}$ & $c_{3}$ & $c_{0}$ & $c_{1}$ & $c_{2}$ & $c_{3}$ \\
\hline & & & & -68.939990 & 315.36807 & -511.21701 & 260.30150 & -13.720239 & 43.260452 & -75.649475 & 35.043575 \\
\hline
\end{tabular}

Table A.14.: Class 14 - Closed to open (>15\%) herbaceous vegetation (grassland, savannas or lichens/mosses). Summary of backscatter statistics and fitting coefficients. 


\begin{tabular}{|c|c|c|c|c|c|c|c|c|c|c|c|}
\hline \multirow{2}{*}{\multicolumn{2}{|c|}{$\begin{array}{c}\text { Class } 15 \\
\text { Continent }\end{array}$}} & \multicolumn{10}{|c|}{ Sparse $(<15 \%)$ vegetation } \\
\hline & & \multicolumn{10}{|c|}{ Asia } \\
\hline \multicolumn{2}{|c|}{ Season } & \multicolumn{5}{|c|}{ Winter } & \multicolumn{5}{|c|}{ Summer } \\
\hline Interval & $\mathrm{E}[\theta]$ & Nsamples & $\mathrm{E}\left[\beta^{0}\right]$ & $\mathrm{E}\left[\beta^{0}\right]-\sigma_{\beta^{0}}$ & $\mathrm{E}\left[\beta^{0}\right]+\sigma_{\beta^{0}}$ & $\mathrm{E}\left[\beta^{0}\right]_{w}$ & Nsamples & $\mathrm{E}\left[\beta^{0}\right]$ & $\mathrm{E}\left[\beta^{0}\right]-\sigma_{\beta^{0}}$ & $\mathrm{E}\left[\beta^{0}\right]+\sigma_{\beta^{0}}$ & $\mathrm{E}\left[\beta^{0}\right]_{w}$ \\
\hline & {$\left[{ }^{\circ}\right]$} & & {$[\mathrm{dB}]$} & {$[\mathrm{dB}]$} & {$[\mathrm{dB}]$} & {$[\mathrm{dB}]$} & & {$[\mathrm{dB}]$} & {$[\mathrm{dB}]$} & {$[\mathrm{dB}]$} & {$[\mathrm{dB}]$} \\
\hline 1 & 25 & 0 & & & & & 1856154 & -5.15 & -10.05 & -2.90 & -5.71 \\
\hline 2 & 27 & 0 & & & & & 4349973 & -5.93 & -11.29 & -3.60 & -8.68 \\
\hline 3 & 29 & 3740706 & -4.85 & -8.78 & -2.83 & -5.04 & 3078077 & -6.89 & -11.37 & -4.73 & -8.95 \\
\hline 4 & 31 & 20366635 & -6.32 & -10.48 & -4.23 & -5.01 & 5401367 & -6.77 & -10.72 & -4.74 & -5.96 \\
\hline 5 & 33 & $1.3396128 \mathrm{e}+08$ & -7.28 & -11.49 & -5.19 & -8.01 & 52927448 & -7.45 & -10.56 & -5.66 & -8.28 \\
\hline 6 & 35 & $3.0581235 \mathrm{e}+08$ & -7.83 & -11.67 & -5.83 & -9.13 & $4.6783440 \mathrm{e}+08$ & -7.81 & -10.04 & -6.35 & -8.52 \\
\hline 7 & 37 & $2.7824050 \mathrm{e}+08$ & -8.12 & -11.41 & -6.26 & -8.91 & $1.3250268 \mathrm{e}+09$ & -8.18 & -10.64 & -6.62 & -8.59 \\
\hline 8 & 39 & $3.1802408 \mathrm{e}+08$ & -8.32 & -11.76 & -6.43 & -8.92 & $1.6169411 \mathrm{e}+09$ & -8.55 & -11.14 & -6.94 & -9.28 \\
\hline 9 & 41 & $3.0956421 \mathrm{e}+08$ & -8.84 & -12.18 & -6.98 & -9.29 & $1.5571352 \mathrm{e}+09$ & -9.12 & -11.95 & -7.42 & -9.99 \\
\hline 10 & 43 & $2.9262161 \mathrm{e}+08$ & -9.39 & -12.71 & -7.53 & -10.06 & $1.1916752 \mathrm{e}+09$ & -9.35 & -12.31 & -7.61 & -10.20 \\
\hline 11 & 45 & $3.4363483 \mathrm{e}+08$ & -10.36 & -14.06 & -8.39 & -11.87 & $6.3188748 \mathrm{e}+08$ & -9.62 & -12.68 & -7.85 & -10.98 \\
\hline 12 & 47 & $2.0049371 \mathrm{e}+08$ & -10.87 & -14.98 & -8.80 & -13.27 & $1.4355132 \mathrm{e}+08$ & -9.88 & -13.06 & -8.07 & -9.82 \\
\hline \multirow[t]{5}{*}{13} & 49 & 7831364 & -10.10 & -14.67 & -7.92 & -9.21 & 4546767 & -9.34 & -13.58 & -7.23 & -8.47 \\
\hline & & & & \multicolumn{8}{|c|}{ Fitting Coefficients } \\
\hline & & & & \multicolumn{4}{|c|}{ Winter } & \multicolumn{4}{|c|}{ Summer } \\
\hline & & & & $c_{0}$ & $c_{1}$ & $c_{2}$ & $c_{3}$ & $c_{0}$ & $c_{1}$ & $c_{2}$ & $c_{3}$ \\
\hline & & & & -26.034094 & 165.36182 & -349.45299 & 208.06034 & -30.559233 & 143.48287 & -271.14551 & 155.43104 \\
\hline
\end{tabular}

Table A.15.: Class 15 - Sparse $(<15 \%)$ vegetation. Summary of backscatter statistics and fitting coefficients.

\begin{tabular}{|c|c|c|c|c|c|c|c|c|c|c|c|}
\hline \multirow{2}{*}{\multicolumn{2}{|c|}{$\begin{array}{c}\text { Class 16 } \\
\text { Continent } \\
\end{array}$}} & \multicolumn{10}{|c|}{ Closed to open (>15\%) broadleaved forest regularly flooded (semi-permanently or temporarily) - Fresh or brackish water } \\
\hline & & \multicolumn{10}{|c|}{$\begin{array}{ll} & \text { South America } \\
\end{array}$} \\
\hline \multicolumn{2}{|c|}{ Season } & \multicolumn{5}{|c|}{ Winter } & \multicolumn{5}{|c|}{ Summer } \\
\hline \multirow[t]{2}{*}{ Interval } & $\mathrm{E}[\theta]$ & Nsamples & $\mathrm{E}\left[\beta^{0}\right]$ & $\mathrm{E}\left[\beta^{0}\right]-\sigma_{\beta^{0}}$ & $\mathrm{E}\left[\beta^{0}\right]+\sigma_{\beta^{0}}$ & $\mathrm{E}\left[\beta^{0}\right]_{w}$ & Nsamples & $\mathrm{E}\left[\beta^{0}\right]$ & $\mathrm{E}\left[\beta^{0}\right]-\sigma_{\beta^{0}}$ & $\mathrm{E}\left[\beta^{0}\right]+\sigma_{\beta^{0}}$ & $\mathrm{E}\left[\beta^{0}\right]_{w}$ \\
\hline & {$\left[{ }^{\circ}\right]$} & & {$[\mathrm{dB}]$} & {$[\mathrm{dB}]$} & {$[\mathrm{dB}]$} & {$[\mathrm{dB}]$} & & {$[\mathrm{dB}]$} & {$[\mathrm{dB}]$} & {$[\mathrm{dB}]$} & {$[\mathrm{dB}]$} \\
\hline 1 & 25 & 1617 & -3.48 & -6.16 & -1.83 & -3.20 & 0 & & & & \\
\hline 2 & 27 & 158 & -2.57 & -4.93 & -1.05 & -2.57 & 133908 & -2.73 & -6.45 & -0.76 & -2.60 \\
\hline 3 & 29 & 6994041 & -3.83 & -6.16 & -2.33 & -3.60 & 7422852 & -3.56 & -5.58 & -2.19 & -2.82 \\
\hline 4 & 31 & 17979468 & -3.91 & -6.26 & -2.40 & -3.50 & 22545732 & -3.85 & -5.87 & -2.47 & -3.37 \\
\hline 5 & 33 & 25646025 & -4.13 & -6.32 & -2.68 & -3.73 & 38615506 & -4.05 & -6.01 & -2.70 & -3.57 \\
\hline 6 & 35 & 24895945 & -4.43 & -6.55 & -3.01 & -4.11 & 46840451 & -4.45 & -6.40 & -3.11 & -4.07 \\
\hline 7 & 37 & 27695779 & -4.54 & -6.68 & -3.11 & -4.24 & 49511899 & -4.74 & -6.74 & -3.38 & -4.39 \\
\hline 8 & 39 & 34653237 & -4.77 & -6.90 & -3.34 & -4.45 & 46836994 & -5.00 & -7.01 & -3.63 & -4.62 \\
\hline 9 & 41 & 53584521 & -5.91 & -8.14 & -4.44 & -5.10 & 44672643 & -5.58 & -7.82 & -4.11 & -5.01 \\
\hline 10 & 43 & 85380588 & -5.72 & -7.68 & -4.38 & -5.38 & 28500421 & -5.90 & -8.03 & -4.48 & -5.57 \\
\hline 11 & 45 & 61769485 & -6.28 & -8.27 & -4.92 & -6.06 & 20842475 & -6.29 & -8.45 & -4.86 & -6.05 \\
\hline 12 & 47 & 42860137 & -6.78 & -8.84 & -5.39 & -6.49 & 31530554 & -6.39 & -8.48 & -4.98 & -6.00 \\
\hline \multirow[t]{5}{*}{13} & 49 & 7994599 & -7.07 & -8.92 & -5.78 & -6.87 & 7351216 & -6.45 & -8.57 & -5.03 & -6.10 \\
\hline & & & & \multicolumn{8}{|c|}{ Fitting Coefficients } \\
\hline & & & & \multicolumn{4}{|c|}{ Winter } & \multicolumn{4}{|c|}{ Summer } \\
\hline & & & & $c_{0}$ & $c_{1}$ & $c_{2}$ & $c_{3}$ & $c_{0}$ & $c_{1}$ & $c_{2}$ & $c_{3}$ \\
\hline & & & & -22.768736 & 101.50967 & -162.10968 & 76.335245 & -10.869555 & 54.694239 & -105.06018 & 55.562198 \\
\hline
\end{tabular}

Table A.16.: Class 16 - Closed to open (>15\%) broadleaved forest regularly flooded (semi-permanently or temporarily) - Fresh or brackish water. Summary of backscatter statistics and fitting coefficients. 


\begin{tabular}{|c|c|c|c|c|c|c|c|c|c|c|c|}
\hline \multirow{2}{*}{\multicolumn{2}{|c|}{$\begin{array}{c}\text { Class } 17 \\
\text { Continent }\end{array}$}} & \multicolumn{10}{|c|}{ Closed (>40\%) broadleaved forest or shrubland permanently flooded - Saline or brackish water } \\
\hline & & \multicolumn{10}{|c|}{ Asia } \\
\hline \multicolumn{2}{|c|}{ Season } & \multicolumn{5}{|c|}{ Winter } & \multicolumn{5}{|c|}{ Summer } \\
\hline \multirow[t]{2}{*}{ Interval } & $\mathrm{E}[\theta]$ & Nsamples & $\mathrm{E}\left[\beta^{0}\right]$ & $\mathrm{E}\left[\beta^{0}\right]-\sigma_{\beta^{0}}$ & $\mathrm{E}\left[\beta^{0}\right]+\sigma_{\beta^{0}}$ & $\mathrm{E}\left[\beta^{0}\right]_{w}$ & Nsamples & $\mathrm{E}\left[\beta^{0}\right]$ & $\mathrm{E}\left[\beta^{0}\right]-\sigma_{\beta^{0}}$ & $\mathrm{E}\left[\beta^{0}\right]+\sigma_{\beta^{0}}$ & $\mathrm{E}\left[\beta^{0}\right]_{w}$ \\
\hline & {$\left[{ }^{\circ}\right]$} & & {$[\mathrm{dB}]$} & {$[\mathrm{dB}]$} & [dB] & {$[\mathrm{dB}]$} & & {$[\mathrm{dB}]$} & {$[\mathrm{dB}]$} & {$[\mathrm{dB}]$} & {$[\mathrm{dB}]$} \\
\hline 1 & 25 & 0 & & & & & 0 & & & & \\
\hline 2 & 27 & 0 & & & & & 15441 & -3.61 & -5.94 & -2.10 & -3.61 \\
\hline 3 & 29 & 1392428 & -4.19 & -6.82 & -2.56 & -3.11 & 263024 & -3.61 & -5.88 & -2.13 & -2.80 \\
\hline 4 & 31 & 4544079 & -4.46 & -7.42 & -2.71 & -3.22 & 1776378 & -4.14 & -7.21 & -2.35 & -3.00 \\
\hline 5 & 33 & 4052619 & -4.68 & -7.67 & -2.93 & -3.23 & 3502136 & -4.02 & -6.83 & -2.33 & -2.79 \\
\hline 6 & 35 & 8093374 & -4.76 & -7.45 & -3.12 & -3.73 & 7893314 & -4.53 & -7.25 & -2.87 & -3.43 \\
\hline 7 & 37 & 6174382 & -5.18 & -7.78 & -3.57 & -3.91 & 14751354 & -4.75 & -7.14 & -3.21 & -3.73 \\
\hline 8 & 39 & 3616729 & -5.53 & -8.08 & -3.93 & -3.98 & 16489989 & -5.19 & -7.67 & -3.62 & -3.77 \\
\hline 9 & 41 & 2951456 & -5.44 & -8.02 & -3.84 & -4.45 & 16987482 & -5.78 & -8.43 & -4.14 & -4.30 \\
\hline 10 & 43 & 3828094 & -6.01 & -8.54 & -4.42 & -4.80 & 11947094 & -6.34 & -9.33 & -4.59 & -4.78 \\
\hline 11 & 45 & 4849310 & -6.60 & -9.84 & -4.76 & -5.34 & 8342504 & -6.87 & -9.87 & -5.12 & -5.57 \\
\hline 12 & 47 & 5386774 & -7.09 & -10.13 & -5.33 & -5.86 & 6966151 & -6.98 & -9.80 & -5.28 & -5.85 \\
\hline \multirow[t]{5}{*}{13} & 49 & 1110994 & -7.46 & -9.95 & -5.89 & -6.14 & 535142 & -6.96 & -10.02 & -5.18 & -5.19 \\
\hline & & & & \multicolumn{8}{|c|}{ Fitting Coefficients } \\
\hline & & & & \multicolumn{4}{|c|}{ Winter } & \multicolumn{4}{|c|}{ Summer } \\
\hline & & & & $c_{0}$ & $c_{1}$ & $c_{2}$ & $c_{3}$ & $c_{0}$ & $c_{1}$ & $c_{2}$ & $c_{3}$ \\
\hline & & & & -10.872983 & 36.431944 & -49.575504 & 15.593474 & -74.568715 & 335.68452 & -508.74729 & 246.52125 \\
\hline
\end{tabular}

Table A.17.: Class 17 - Closed (>40\%) broadleaved forest or shrubland permanently flooded - Saline or brackish water. Summary of backscatter statistics and fitting coefficients.

\begin{tabular}{|c|c|c|c|c|c|c|c|c|c|c|c|}
\hline \multirow{2}{*}{\multicolumn{2}{|c|}{$\begin{array}{c}\text { Class } 18 \\
\text { Continent }\end{array}$}} & \multicolumn{10}{|c|}{ Closed to open (>15\%) grassland or woody vegetation on regularly flooded or waterlogged soil - Fresh, brackish or saline water } \\
\hline & & \multicolumn{10}{|c|}{$\begin{array}{ll}\text { South America } \\
\end{array}$} \\
\hline \multicolumn{2}{|c|}{ Season } & \multicolumn{5}{|c|}{ Winter } & \multicolumn{5}{|c|}{ Summer } \\
\hline Interval & $\mathrm{E}[\theta]$ & Nsamples & $\mathrm{E}\left[\beta^{0}\right]$ & $\mathrm{E}\left[\beta^{0}\right]-\sigma_{\beta^{0}}$ & $\mathrm{E}\left[\beta^{0}\right]+\sigma_{\beta^{0}}$ & $\mathrm{E}\left[\beta^{0}\right]_{w}$ & Nsamples & $\mathrm{E}\left[\beta^{0}\right]$ & $\mathrm{E}\left[\beta^{0}\right]-\sigma_{\beta^{0}}$ & $\mathrm{E}\left[\beta^{0}\right]+\sigma_{\beta^{0}}$ & $\mathrm{E}\left[\beta^{0}\right]_{w}$ \\
\hline & {$\left[{ }^{\circ}\right]$} & & {$[\mathrm{dB}]$} & {$[\mathrm{dB}]$} & {$[\mathrm{dB}]$} & {$[\mathrm{dB}]$} & & {$[\mathrm{dB}]$} & {$[\mathrm{dB}]$} & {$[\mathrm{dB}]$} & {$[\mathrm{dB}]$} \\
\hline 1 & 25 & 0 & & & & & 0 & & & & \\
\hline 2 & 27 & 0 & & & & & 190600 & -2.82 & -6.52 & -0.85 & -2.62 \\
\hline 3 & 29 & 1884707 & -4.31 & -7.03 & -2.65 & -3.81 & 2265095 & -4.48 & -7.68 & -2.66 & -4.04 \\
\hline 4 & 31 & 4692973 & -4.42 & -7.43 & -2.66 & -4.10 & 12949613 & -4.47 & -7.49 & -2.70 & -4.38 \\
\hline 5 & 33 & 6397135 & -4.59 & -7.69 & -2.80 & -4.27 & 27049284 & -4.83 & -7.60 & -3.16 & -4.48 \\
\hline 6 & 35 & 8101427 & -5.19 & -8.16 & -3.44 & -4.81 & 36662474 & -5.37 & -8.18 & -3.68 & -4.94 \\
\hline 7 & 37 & 8975364 & -5.40 & -8.45 & -3.63 & -4.89 & 38309998 & -5.60 & -8.38 & -3.92 & -5.13 \\
\hline 8 & 39 & 10268158 & -5.64 & -8.74 & -3.85 & -5.24 & 44093743 & -6.05 & -8.86 & -4.36 & -5.40 \\
\hline 9 & 41 & 12330527 & -6.36 & -9.30 & -4.62 & -5.88 & 35360965 & -6.80 & -9.59 & -5.11 & -5.91 \\
\hline 10 & 43 & 25401494 & -6.85 & -9.78 & -5.11 & -6.26 & 22805382 & -7.29 & -10.16 & -5.58 & -6.51 \\
\hline 11 & 45 & 32290991 & -7.21 & -10.10 & -5.49 & -6.69 & 11073975 & -7.55 & -10.50 & -5.80 & -7.00 \\
\hline 12 & 47 & 28707565 & -7.50 & -10.40 & -5.78 & -7.15 & 6815800 & -7.25 & -10.20 & -5.51 & -6.72 \\
\hline \multirow[t]{5}{*}{13} & 49 & 4267718 & -7.57 & -10.06 & -6.00 & -7.42 & 3385227 & -7.03 & -9.90 & -5.32 & -6.90 \\
\hline & & & & \multicolumn{8}{|c|}{ Fitting Coefficients } \\
\hline & & & & \multicolumn{4}{|c|}{ Winter } & \multicolumn{4}{|c|}{ Summer } \\
\hline & & & & $c_{0}$ & $c_{1}$ & $c_{2}$ & $c_{3}$ & $c_{0}$ & $c_{1}$ & $c_{2}$ & $c_{3}$ \\
\hline & & & & -14.706707 & 58.442292 & -94.276990 & 41.879690 & 30.735944 & -136.84205 & 177.41915 & -80.883958 \\
\hline
\end{tabular}

Table A.18.: Class 18 - Closed to open (>15\%) grassland or woody vegetation on regularly flooded or waterlogged soil - Fresh, brackish or saline water. Summary of backscatter statistics and fitting coefficients. 


\begin{tabular}{|c|c|c|c|c|c|c|c|c|c|c|c|}
\hline \multirow{2}{*}{\multicolumn{2}{|c|}{$\begin{array}{c}\text { Class } 19 \\
\text { Continent }\end{array}$}} & \multicolumn{10}{|c|}{ Artificial surfaces and associated areas (Urban areas $>\mathbf{5 0 \%}$ ) } \\
\hline & & \multicolumn{10}{|c|}{$\begin{array}{ll}\text { Europe } \\
\end{array}$} \\
\hline \multicolumn{2}{|c|}{ Season } & \multicolumn{5}{|c|}{ Winter } & \multicolumn{5}{|c|}{ Summer } \\
\hline \multirow[t]{2}{*}{ Interval } & $\mathrm{E}[\theta]$ & Nsamples & $\mathrm{E}\left[\beta^{0}\right]$ & $\mathrm{E}\left[\beta^{0}\right]-\sigma_{\beta^{0}}$ & $\mathrm{E}\left[\beta^{0}\right]+\sigma_{\beta^{0}}$ & $\mathrm{E}\left[\beta^{0}\right]_{w}$ & Nsamples & $\mathrm{E}\left[\beta^{0}\right]$ & $\mathrm{E}\left[\beta^{0}\right]-\sigma_{\beta^{0}}$ & $\mathrm{E}\left[\beta^{0}\right]+\sigma_{\beta^{0}}$ & $\mathrm{E}\left[\beta^{0}\right]_{w}$ \\
\hline & {$\left[{ }^{\circ}\right]$} & & {$[\mathrm{dB}]$} & {$[\mathrm{dB}]$} & {$[\mathrm{dB}]$} & {$[\mathrm{dB}]$} & & {$[\mathrm{dB}]$} & {$[\mathrm{dB}]$} & {$[\mathrm{dB}]$} & {$[\mathrm{dB}]$} \\
\hline 1 & 25 & 0 & & & & & 0 & & & & \\
\hline 2 & 27 & 0 & & & & & 0 & & & & \\
\hline 3 & 29 & 0 & & & & & 0 & & & & \\
\hline 4 & 31 & 31039 & -2.34 & -22.05 & 0.6 & -2.57 & 100912 & -3.76 & -14.21 & -0.95 & -3.27 \\
\hline 5 & 33 & 6164707 & -4.13 & -11.79 & -1.51 & -3.39 & 1079573 & -3.87 & -10.61 & -1.34 & -3.01 \\
\hline 6 & 35 & 18285124 & -4.53 & -13.76 & -1.79 & -3.71 & 6193245 & -5.41 & -12.64 & -2.83 & -4.10 \\
\hline 7 & 37 & 11726590 & -5.11 & -12.91 & -2.48 & -4.20 & 13207241 & -5.53 & -13.26 & -2.90 & -4.21 \\
\hline 8 & 39 & 8150345 & -5.54 & -13.73 & -2.87 & -4.74 & 19367347 & -5.50 & -14.11 & -2.80 & -4.45 \\
\hline 9 & 41 & 8343456 & -5.96 & -13.74 & -3.33 & -5.08 & 30546456 & -5.86 & -15.57 & -3.08 & -4.81 \\
\hline 10 & 43 & 11086747 & -6.45 & -14.76 & -3.77 & -5.45 & 29954113 & -6.13 & -16.56 & -3.33 & -5.11 \\
\hline 11 & 45 & 18394389 & -6.73 & -14.85 & -4.07 & -5.55 & 22855430 & -6.53 & -16.66 & -3.74 & -5.51 \\
\hline 12 & 47 & 8544561 & -7.11 & -16.81 & -4.34 & -5.81 & 7785366 & -6.79 & -17.53 & -3.96 & -5.79 \\
\hline \multirow[t]{5}{*}{13} & 49 & 150501 & -8.51 & -27.89 & -5.52 & -6.97 & 9167 & -7.31 & -11.21 & -5.29 & -5.94 \\
\hline & & & & \multicolumn{8}{|c|}{ Fitting Coefficients } \\
\hline & & & & \multicolumn{4}{|c|}{ Winter } & \multicolumn{4}{|c|}{ Summer } \\
\hline & & & & $c_{0}$ & $c_{1}$ & $c_{2}$ & $c_{3}$ & $c_{0}$ & $c_{1}$ & $c_{2}$ & $c_{3}$ \\
\hline & & & & 73.869851 & -319.01522 & 441.88470 & -209.59590 & -27.381946 & 122.42496 & -195.06177 & 94.985167 \\
\hline
\end{tabular}

Table A.19.: Class 19 - Artificial surfaces and associated areas (Urban areas $>50 \%$ ). Summary of backscatter statistics and fitting coefficients.

\begin{tabular}{|c|c|c|c|c|c|c|c|c|c|c|c|}
\hline \multirow{2}{*}{\multicolumn{2}{|c|}{$\begin{array}{c}\text { Class 20 } \\
\text { Continent }\end{array}$}} & \multicolumn{10}{|c|}{ Bare areas } \\
\hline & & \multicolumn{10}{|c|}{ Africa } \\
\hline \multicolumn{2}{|c|}{ Season } & \multicolumn{5}{|c|}{ Winter } & \multicolumn{5}{|c|}{ Summer } \\
\hline Interval & $\mathrm{E}[\theta]$ & Nsamples & $\mathrm{E}\left[\beta^{0}\right]$ & $\mathrm{E}\left[\beta^{0}\right]-\sigma_{\beta^{0}}$ & $\mathrm{E}\left[\beta^{0}\right]+\sigma_{\beta^{0}}$ & $\mathrm{E}\left[\beta^{0}\right]_{w}$ & Nsamples & $\mathrm{E}\left[\beta^{0}\right]$ & $\mathrm{E}\left[\beta^{0}\right]-\sigma_{\beta^{0}}$ & $\mathrm{E}\left[\beta^{0}\right]+\sigma_{\beta^{0}}$ & $\mathrm{E}\left[\beta^{0}\right]_{w}$ \\
\hline & {$\left[{ }^{\circ}\right]$} & & {$[\mathrm{dB}]$} & {$[\mathrm{dB}]$} & {$[\mathrm{dB}]$} & {$[\mathrm{dB}]$} & & {$[\mathrm{dB}]$} & {$[\mathrm{dB}]$} & {$[\mathrm{dB}]$} & {$[\mathrm{dB}]$} \\
\hline 1 & 25 & $6.6401936 \mathrm{e}+08$ & -9.61 & -15.73 & -7.17 & -10.07 & $8.9476799 \mathrm{e}+08$ & -9.09 & -14.89 & -6.69 & -10.15 \\
\hline 2 & 27 & $2.3438050 \mathrm{e}+08$ & -11.13 & -17.44 & -8.66 & -11.65 & $1.2604028 \mathrm{e}+09$ & -10.36 & -15.98 & -7.99 & -11.18 \\
\hline 3 & 29 & $7.0282588 \mathrm{e}+08$ & -10.05 & -14.39 & -7.93 & -11.54 & $9.9490939 \mathrm{e}+08$ & -10.41 & -15.81 & -8.07 & -11.31 \\
\hline 4 & 31 & $1.4653708 \mathrm{e}+09$ & -9.79 & -14.66 & -7.55 & -11.66 & $5.3032284 \mathrm{e}+08$ & -10.03 & -14.77 & -7.82 & -10.29 \\
\hline 5 & 33 & $1.9272130 \mathrm{e}+09$ & -10.07 & -14.81 & -7.86 & -11.90 & $2.1569572 \mathrm{e}+08$ & -9.57 & -13.15 & -7.63 & -9.99 \\
\hline 6 & 35 & $1.4694014 \mathrm{e}+09$ & -10.39 & -15.04 & -8.20 & -11.64 & $1.1145345 \mathrm{e}+08$ & -9.48 & -12.27 & -7.80 & -9.93 \\
\hline 7 & 37 & $6.9350847 \mathrm{e}+08$ & -10.36 & -14.51 & -8.27 & -10.93 & $4.4795450 \mathrm{e}+08$ & -10.50 & -13.66 & -8.70 & -12.00 \\
\hline 8 & 39 & $5.0186950 \mathrm{e}+08$ & -10.44 & -15.17 & -8.22 & -11.08 & $1.3625265 \mathrm{e}+09$ & -11.04 & -14.75 & -9.06 & -12.30 \\
\hline 9 & 41 & $3.9725080 \mathrm{e}+08$ & -10.56 & -15.21 & -8.36 & -11.35 & $2.7378803 e+09$ & -11.77 & -15.87 & -9.70 & -13.10 \\
\hline 10 & 43 & $2.9137475 \mathrm{e}+08$ & -10.50 & -15.04 & -8.33 & -11.12 & $3.5581712 \mathrm{e}+09$ & -12.43 & -16.41 & -10.39 & -13.32 \\
\hline 11 & 45 & $3.0441983 e+08$ & -11.38 & -15.03 & -9.42 & -12.21 & $3.0580900 \mathrm{e}+09$ & -13.05 & -16.94 & -11.03 & -13.54 \\
\hline 12 & 47 & $1.0837014 \mathrm{e}+09$ & -12.81 & -16.64 & -10.81 & -13.91 & $1.4978926 \mathrm{e}+09$ & -13.17 & -17.08 & -11.15 & -13.32 \\
\hline \multirow[t]{5}{*}{13} & 49 & $3.3904753 \mathrm{e}+08$ & -13.53 & -17.01 & -11.63 & -14.21 & 85585876 & -13.08 & -17.12 & -11.03 & -12.75 \\
\hline & & & & \multicolumn{8}{|c|}{ Fitting Coefficients } \\
\hline & & & & \multicolumn{4}{|c|}{ Winter } & \multicolumn{4}{|c|}{ Summer } \\
\hline & & & & $c_{0}$ & $c_{1}$ & $c_{2}$ & $c_{3}$ & $c_{0}$ & $c_{1}$ & $c_{2}$ & $c_{3}$ \\
\hline & & & & 73.406124 & -423.55036 & 693.43802 & -372.69966 & -75.980085 & 326.81177 & -523.35510 & 265.86568 \\
\hline
\end{tabular}

Table A.20.: Class 20 - Bare areas. Summary of backscatter statistics and fitting coefficients. 